\title{
Medidas de dependência local para séries temporais
}

\author{
Sumaia Abdel Latif
}

TESE APRESENTADA

$\mathrm{AO}$

INSTITUTO DE MATEMÁTICA E ESTATÍSTICA

DA

UNIVERSIDADE DE SÃo PAULO

PARA

OBTENÇÃO DO TÍTULO

$\mathrm{DE}$

DOUTORA EM CIÊNCIAS

Área de Concentração: Estatística

Orientador: Prof. Dr. Pedro Alberto Morettin

São Paulo, janeiro de 2008 



\section{Agradecimentos}

Registro aqui o meu agradecimento ao Prof. Dr Pedro A. Morettin pelo privilégio que me concedeu ao concordar em me orientar nesta tese, pelos seus ensinamentos, e também pela disposição e atenção com que sempre me atendeu.

Agradeço aos meus amigos pelo incentivo inicial, e também pelo convívio ao longo desta jornada.

À minha família, sou grata pelo apoio e compreensão contínuos. 


\section{Resumo}

Diferente das medidas de associação global (coeficiente de correlação linear de Pearson, de Spearman, tau de Kendall, por exemplo), as medidas de dependência local descrevem o comportamento da dependência localmente em diferentes regiões.

Nesta tese, as medidas de dependência local para variáveis aleatórias propostas por Bairamov et al. (2003), Bjerve e Doksum (1993) e Sibuya (1960), são estudadas sob o enfoque de processos estocásticos estacionários bivariados e univariados, neste caso, estudando o comportamento da dependência local ao longo das defasagens da série temporal.

Para as duas primeiras medidas, discutimos as suas propriedades, e estudamos os seus estimadores, além da consistência dos mesmos. Para a medida de Sibuya, além de discutir suas propriedades, propomos três estimadores para variáveis aleatórias e também para séries temporais, verificando a consistência dos mesmos. O comportamento das três medidas locais e dos seus estimadores foram avaliados através de simulações e aplicações a dados reais (neste caso, fizemos uma comparação destas com cópula e densidade cópula).

Palavras-chave: séries temporais, medidas de dependência local 


\begin{abstract}
Unlike global association measures (Pearson's linear correlation coefficient, Spearman's rho, Kendall's tau, for example), local dependence measures describe the behaviour of dependence locally in different regions.

In this thesis, the local dependence measures for random variables proposed by Bairamov et al. (2003), Bjerve and Doksum (1993) and Sibuya (1960), are studied in the context of bivariate and univariate stationary stochastic processes, in this case, evaluating the performance of local dependence along time lags.

We discussed the properties and studied the estimators and consistence of the first two measures. As for the Sibuya measure, in addition to discussing its properties, we propose three estimators for the random variables and time series while checking their consistence. The behaviour of the three local measures and their respective estimators was evaluated by simulations and application to real data (in this case, a comparison was drawn with copula and copula density)
\end{abstract}

Keywords: time series, local dependence measures 


\section{Sumário}

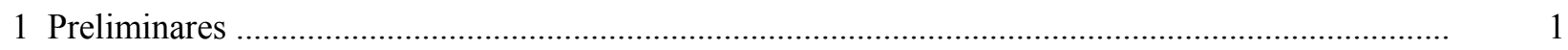

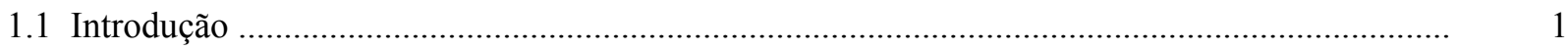

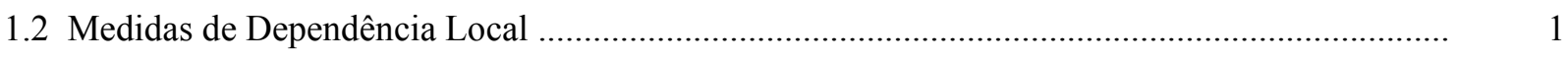

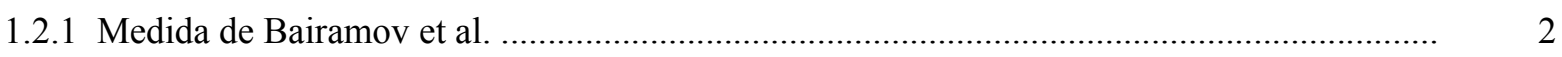

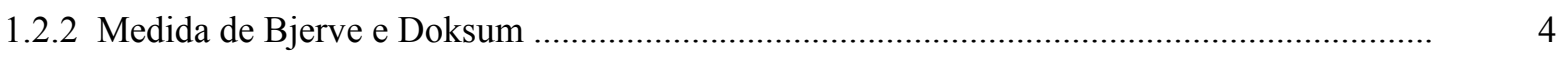

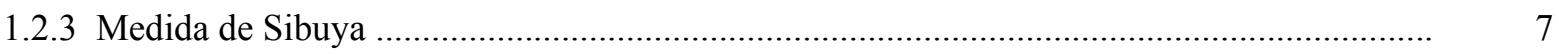

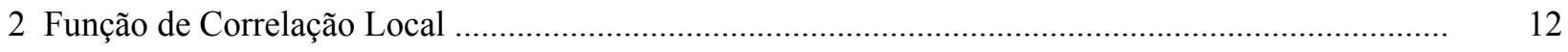

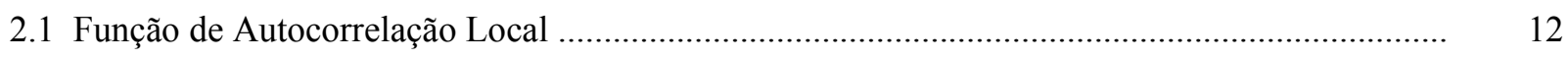

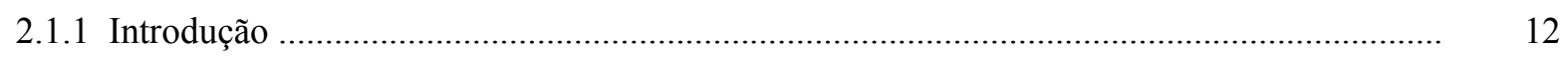

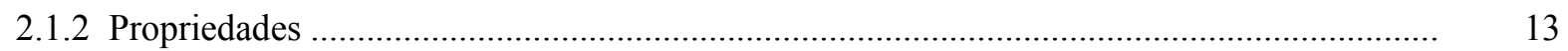

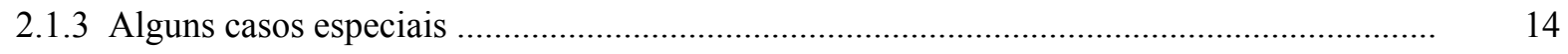

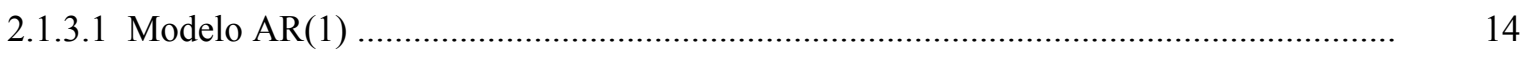

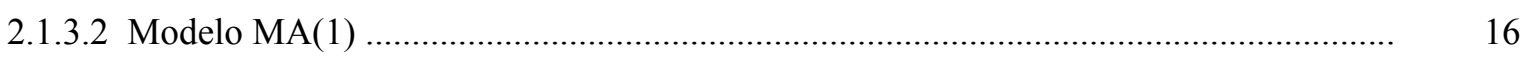

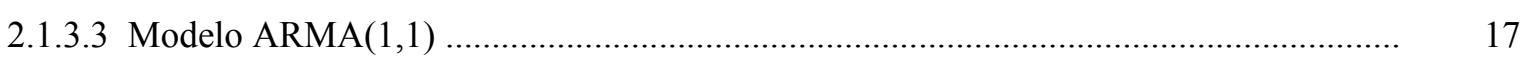

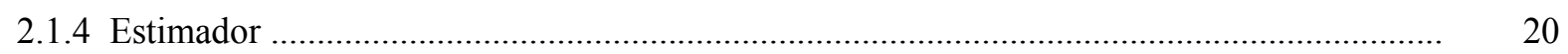

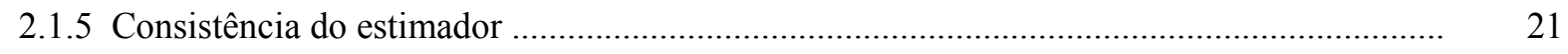

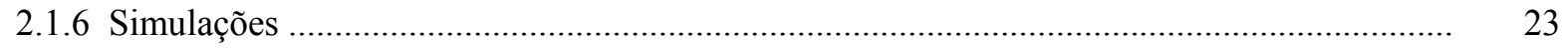

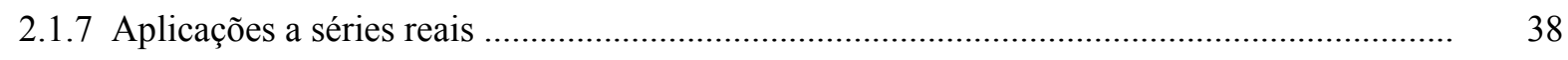

2.2 Função de Correlação Cruzada Contemporânea Local ................................................................ 45

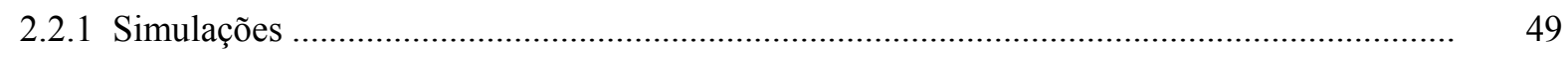

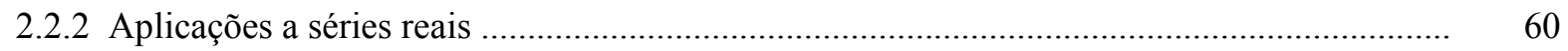

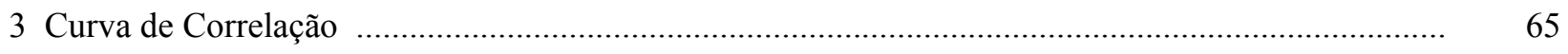

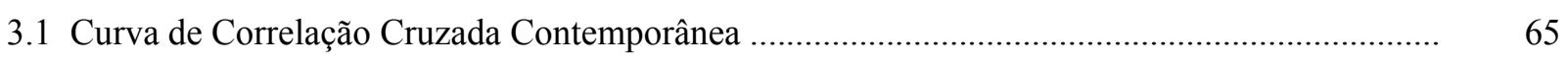

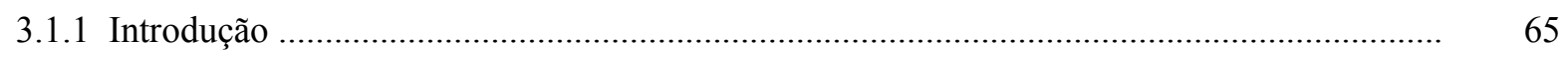

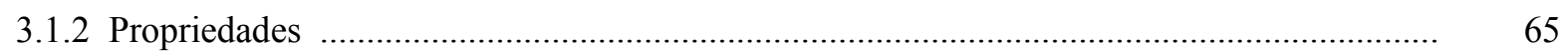

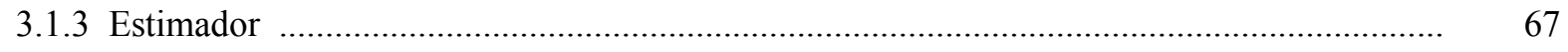

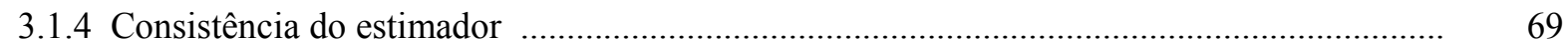

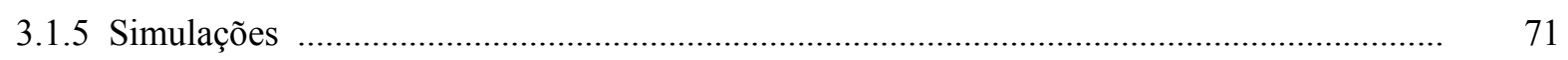

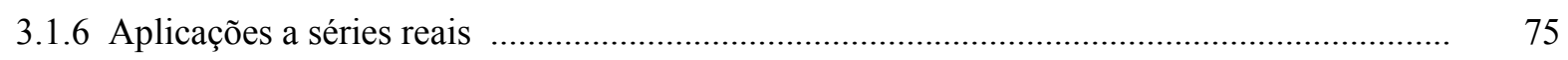

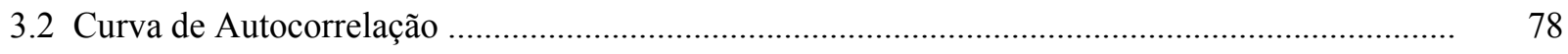

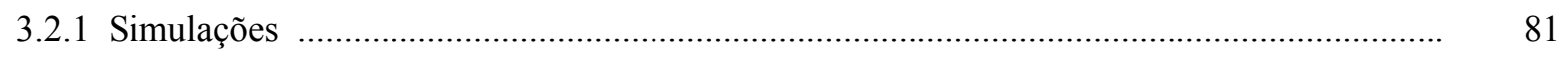

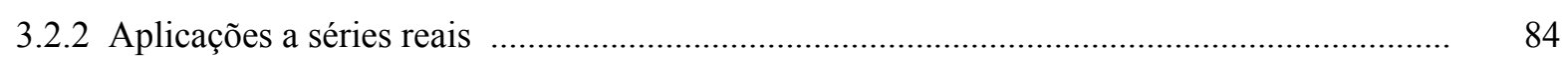




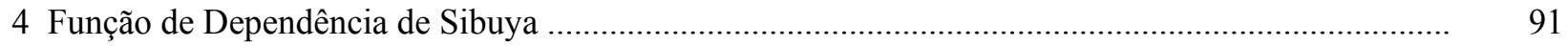

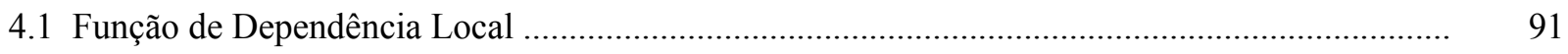

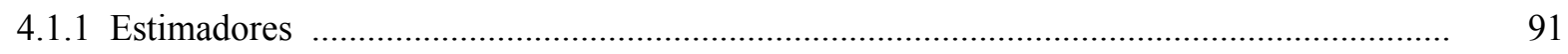

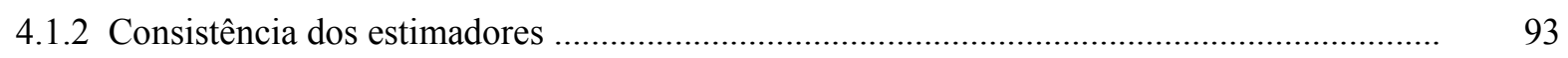

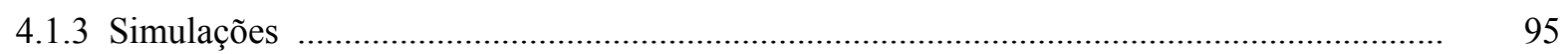

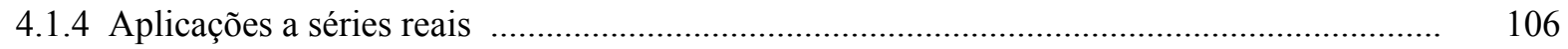

4.2 Função de Dependência Cruzada Contemporânea Local ................................................................ 110

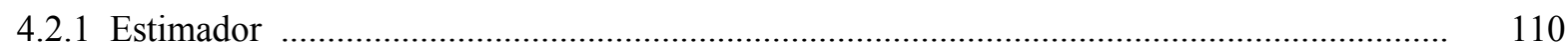

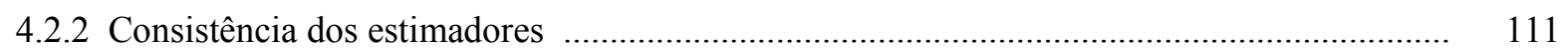

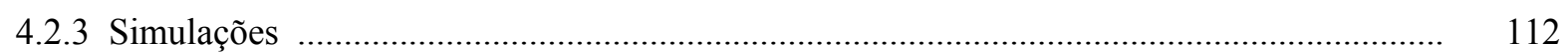

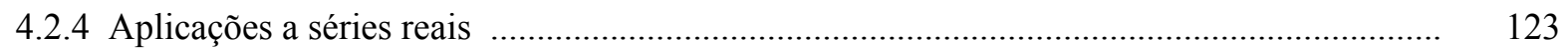

4.3 Função de Autodependência Local ........................................................................................ 127

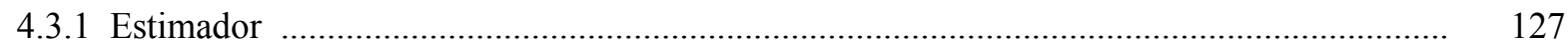

4.3.2 Consistência dos estimadores $\quad$................................................................................. 128

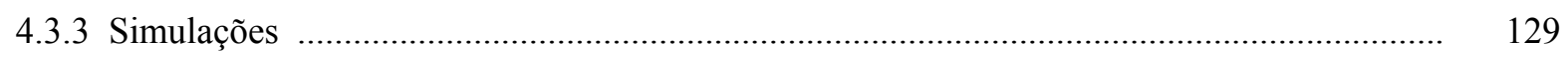

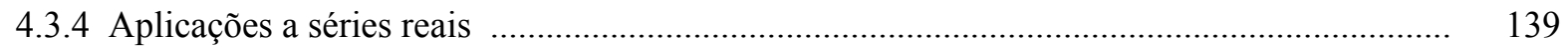

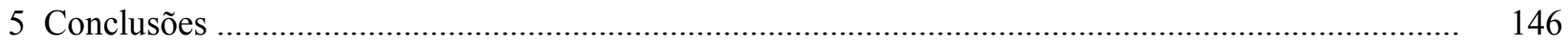

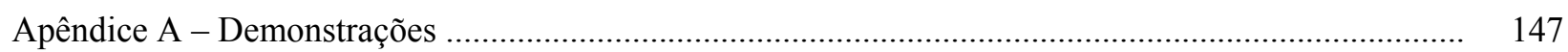

Apêndice B - Simulações adicionais da Função de Correlação Local .................................................... 150

Apêndice C - Simulações adicionais da Curva de Correlação ................................................................ 157

Apêndice D - Simulações adicionais da Função de Dependência de Sibuya .......................................... 160

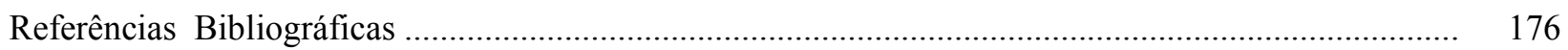




\section{Capítulo 1}

\section{Preliminares}

\subsection{Introdução}

O coeficiente de correlação linear de Pearson mede o grau de associação linear global entre duas variáveis quantitativas, porém, os dados podem apresentar diferentes comportamentos de associação se considerarmos subconjuntos desses dados. Com o objetivo de detectar comportamentos localizados nos dados, medidas locais foram propostas para variáveis aleatórias, como a função de dependência local de Holland e Wang (1987), a curva de correlação de Bjerve e Doksum (1993) e a medida de dependência local de Bairamov et al. (2003). Também, outras propostas para medir a dependência local foram sugeridas por Nelsen (2007), Anjos e Kolev (2005) e Sibuya (1960), para a qual Kolev et al. (2007) desenvolveram propriedades adicionais.

No contexto de processos estocásticos, poucos trabalhos foram desenvolvidos para avaliar medidas de dependência local. Dentre eles, podemos mencionar o estudo de cópulas para séries temporais abordado tanto por Fermanian e Scaillet (2003), que utilizaram estimadores tipo kernel, quanto por Morettin et al. (2006) que utilizaram estimadores tipo ondaletas.

O objetivo deste trabalho é o estudo das medidas de dependência local de Bairamov et al., Bjerve e Doksum e Sibuya sob o enfoque de séries temporais, considerando processos estacionários univariados e bivariados. São discutidas as suas propriedades, de modo a compará-las com as propriedades que uma medida de dependência global deve satisfazer (Rényi, 1959). Para as duas primeiras medidas, discutimos as suas propriedades, e estudamos os seus estimadores, além da consistência dos mesmos. Para a medida de Sibuya, além de discutir suas propriedades, propomos três estimadores para variáveis aleatórias e também para séries temporais, verificando a consistência dos mesmos. O comportamento das três medidas locais e dos seus estimadores foram avaliados através de simulações e aplicações a dados reais (neste caso, fizemos uma comparação destas com cópula e densidade cópula).

\subsection{Medidas de Dependência Local}

Antes de iniciarmos a introdução das medidas de dependência local, segue uma breve introdução às propriedades que uma medida de dependência global deve satisfazer (Rényi, 1959). Sejam $\xi$ e $\eta$ duas variáveis aleatórias definidas num espaço de probabilidades $(\Omega, A, \mathscr{P})$, nenhuma delas sendo constante com probabilidade 1. Seja $\delta(\xi, \eta)$ uma medida de dependência. Considera-se que o intervalo natural de variação da força de dependência é $[0,1]$ em que 1 indica dependência estrita e 0 indica independência. Então, as seguintes propriedades devem estar satisfeitas: 
- $0 \leq \delta(\xi, \eta) \leq 1$;

- $\delta(\xi, \eta)=0 \Leftrightarrow \xi$ e $\eta$ são independentes;

- $\delta(\xi, \eta)=1$ se existe uma dependência estrita entre $\xi$ e $\eta$, isto é, se $\xi=g(\eta)$ ou $\eta=f(\xi)$, em que $f$ e $g$ são funções mensuráveis Borel;

- $\delta(\xi, \eta)$ é definida para qualquer par de v.a. $\xi$ e $\eta$, nenhuma delas sendo constante com probabilidade 1;

- $\delta(\xi, \eta)=\delta(\eta, \xi)$

- $\quad$ se as funções mensuráveis Borel $f(x)$ e $g(x)$ são funções de $R$ em $R, 1-1$, então $\delta(f(\xi), g(\eta))=\delta(\xi, \eta)$;

- se a distribuição conjunta de $\xi$ e $\eta$ é normal, então $\delta(\xi, \eta)=|\rho|$, em que $\rho$ é o coeficiente de correlação linear de Pearson entre $\xi$ e $\eta$.

\subsubsection{Medida de Bairamovet al.}

A medida de dependência local entre duas variáveis aleatórias contínuas $X$ e $Y$ proposta por Bairamov et al. (2003) é dada pela seguinte expressão

$$
H(x, y)=\frac{E[(X-E[X / Y=y])(Y-E[Y / X=x])]}{\sqrt{E\left[(X-E[X / Y=y])^{2}\right]} \sqrt{E\left[(Y-E[Y / X=x])^{2}\right]}}, \forall(x, y) \in S,
$$

a qual refere-se ao conhecido coeficiente de correlação linear de Pearson no qual foi substituída a $E[X]$ por $E[X / Y=y]$ e a $E[Y]$ por $E[Y / X=x]$. Aqui, $S$ denota o suporte de $(X, Y)$.

Analisando-se os termos de $H(x, y)$, podemos verificar facilmente que

$$
\begin{aligned}
& E[(X-E[X / Y=y])(Y-E[Y / X=x])]=\operatorname{Cov}(X, Y)+(E[X]-E[X / Y=y])(E[Y]-E[Y / X=x]), \\
& E\left[(X-E[X / Y=y])^{2}\right]=\operatorname{Var}[X]+(E[X]-E[X / Y=y])^{2}, \\
& E\left[(Y-E[Y / X=x])^{2}\right]=\operatorname{Var}[Y]+(E[Y]-E[Y / X=x])^{2},
\end{aligned}
$$

portanto, essa medida também pode ser escrita na representação alternativa

$$
H(x, y)=\frac{\operatorname{Cov}(X, Y)+\lambda_{X}(y) \lambda_{Y}(x)}{\sqrt{\operatorname{Var}[X]+\lambda_{X}^{2}(y)} \sqrt{\operatorname{Var}[Y]+\lambda_{Y}^{2}(x)}},
$$

em que

$$
\lambda_{X}(y)=E[X]-E[X / Y=y], \lambda_{Y}(x)=E[Y]-E[Y / X=x] \text { e } \operatorname{Cov}(X, Y)=E[(X-E[X])(Y-E[Y])] .
$$

Observa-se que se $X$ e $Y$ são independentes então $\lambda_{X}(y)=\lambda_{Y}(x)=\operatorname{Cov}(X, Y)=0$ e $H(x, y)=0$.

Dividindo-se o numerador e o denominador de $H(x, y)$ acima pelo produto do desvio-padrão de $X$ e desvio-padrão de $Y$, temos

$$
H(x, y)=\frac{\rho_{X Y}+\varphi_{X}(y) \varphi_{Y}(x)}{\sqrt{1+\varphi_{X}^{2}(y)} \sqrt{1+\varphi_{Y}^{2}(x)}},
$$

em que

$$
\varphi_{X}(y)=\frac{E[X]-E[X / Y=y]}{\sqrt{\operatorname{Var}[X]}}, \varphi_{Y}(x)=\frac{E[Y]-E[Y / X=x]}{\sqrt{\operatorname{Var}[Y]}} \text { e } \rho_{X Y}=\frac{\operatorname{Cov}(X, Y)}{\sqrt{\operatorname{Var}[X]} \sqrt{\operatorname{Var}[Y]}}
$$


Na Figura 1.1 a seguir, os gráficos de perspectiva (a) a (d) e os correspondentes gráficos de curvas de nível (e) a (h), mostram o comportamento teórico desta medida, considerando-se um vetor aleatório bivariado com distribuição normal padrão e coeficiente de correlação $+0,80,-0,80,+0,20$ e $-0,20$, nesta ordem. A grade bivariada utilizada corresponde a $98 \%$ da distribuição central de cada marginal.

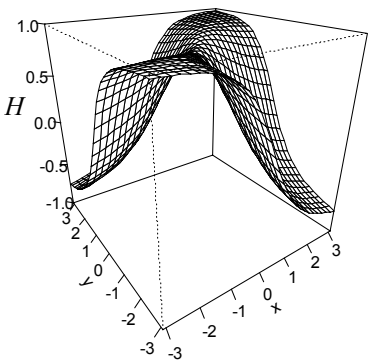

(a)

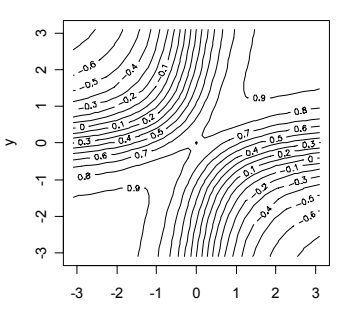

(e)

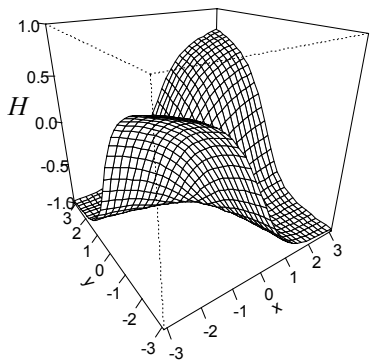

(b)

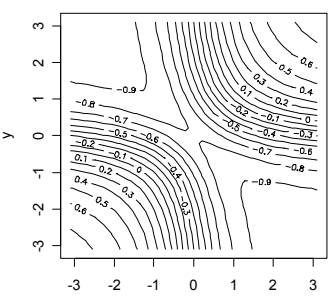

(f)

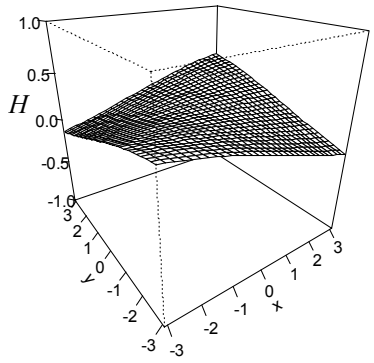

(c)

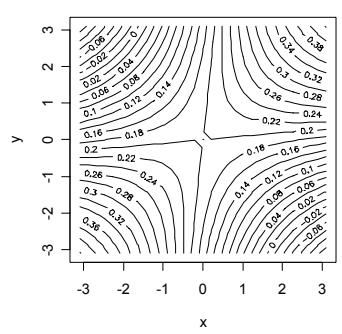

(g)

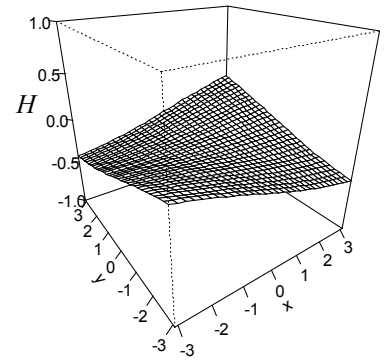

(d)

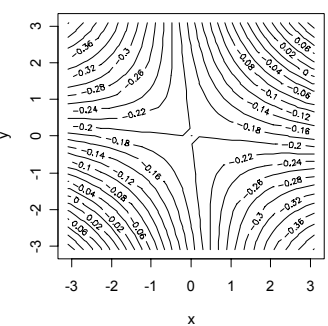

(h)

Figura 1.1 - Gráficos de perspectiva e de curvas de nível da medida de dependência local de Bairamov et al. para $(X, Y)$ com distribuição normal padrão e coeficiente de correlação $\rho=+0,80 \mathrm{em}(\mathrm{a}) \mathrm{e}(\mathrm{e})$,

$$
\rho=-0,80 \text { em (b) e (f), } \rho=+0,20 \text { em (c) e (g), e } \rho=-0,20 \text { em (d) e (h). }
$$

Observamos que:

- $\quad H(x, y)$ refere-se à versão localizada do coeficiente de correlação linear de Pearson $\rho(X, Y)$;

- esta medida de dependência local caracteriza o efeito de $X$ em $Y$ e o efeito de $Y$ em $X$, condicional a $(X, Y)$ estar no ponto $(x, y)$, permitindo a localização de valores das variáveis com associação mais forte ou mais fraca que a associação global;

- a $E[H(X, Y)]$ é aproximadamente igual ao coeficiente de correlação linear de Pearson. Esta aproximação pode ser efetuada através da integração ponderada de $H$ em relação à densidade conjunta $f$ de $(X, Y)$, e o resultado é sempre finito pois $|H(x, y)| \leq 1$. 
Observamos também que a forma e a simetria de $H(x, y)$ para as distribuições simétricas elípticas podem ser encontradas em Kotz e Nadarajah (2003), e para as distribuições de valores extremos em Nadarajah et al. (2003).

Seja $(X, Y)$ um vetor aleatório contínuo com componentes possuindo momentos de segunda ordem finitos e com suporte $S$. Então, $H(x, y)$ satisfaz às seguintes propriedades (Bairamov et al., 2003):

(i) $|H(x, y)| \leq 1, \forall(x, y) \in S$;

(ii) Se $H(x, y)= \pm 1$ para algum $(x, y) \in S$, então $\rho_{X Y} \neq 0$;

(iii) Se $Y=a X+b$ quase certamente, então $H(X, Y)=1 \times \operatorname{sinal}(a)$;

(iv) Se $\rho_{X Y}= \pm 1$ então $H(X, Y)= \pm 1$, quase certamente ;

(v) Se $U=a+b X$ e $V=c+d Y$, com $b d \neq 0$, então $H_{U V}(u, v)=\operatorname{sinal}(b d) H_{X Y}(x, y)$, em que $u=a+b x$ e $v=c+d y$

(vi) Se $X$ e $Y$ são independentes, então $H(x, y)=0, \forall(x, y) \in S$;

(vii) Se $H(x, y)=0, \forall(x, y) \in S$, então $E[X]=E[X / Y=y]$ ou $E[Y]=E[Y / X=x], \forall(x, y) \in S$, e $\rho_{X Y}=0$;

(viii) $\mathrm{O}$ ponto $\left(x^{*}, y^{*}\right)$ satisfazendo $\varphi_{X}\left(y^{*}\right)=\varphi_{Y}\left(x^{*}\right)=0$ é um ponto de sela de $H$, e neste ponto, $H\left(x^{*}, y^{*}\right)=\rho_{X Y}, \operatorname{com}\left(x^{*}, y^{*}\right) \in S ;$

(ix) $H\left(\mu_{X}, \mu_{Y}\right)=\rho_{X Y}$ se $(X, Y)$ tem distribuição normal com média $\left(\mu_{X}, \mu_{Y}\right)^{\prime}$.

Para estimar esta medida, Bairamov et al. (2003) sugerem usar os estimadores usuais para $E[X], E[Y]$, $\operatorname{Var}[X], \operatorname{Var}[Y]$ e $\rho_{X Y}$, e estimadores tipo kernel para as esperanças condicionais.

\subsubsection{Medida de Bjerve e Doksum}

Segundo Doksum et al. (1994), a motivação original para o conceito de correlação local surgiu através de Jack Block do departamento de Psicologia da Universidade da Califórnia em Berkeley, e de Per Gjerde do Conselho de Psicologia da Universidade da Califórnia em Santa Cruz. Eles questionaram como analisar a associação entre duas variáveis aleatórias $X$ e $Y$ cujo diagrama de dispersão apresentava uma forma similar a uma pêra retorcida como na Figura 1.2. A proposta inicial dos autores foi transformar as variáveis originais de modo que elas seguissem aproximadamente um modelo linear para então calcular o coeficiente de correlação linear de Pearson. Porém, Block e Gjerde não ficaram satisfeitos pois o que eles queriam era exatamente quantificar a associação não linear entre as variáveis originais.

Com o objetivo de criar uma medida da força da associação local, deve-se ter o devido cuidado ao se passar do caso linear para o caso geral. Por exemplo, ao utilizar regressão não paramétrica para estimar $E[Y / X=x]$, temos que esta abordagem não leva em consideração a heteroscedasticidade de $Y$ ao longo dos diferentes valores de $X$. Portanto, tal método precisaria ser complementado com uma medida da dispersão de $Y / X=x$. 


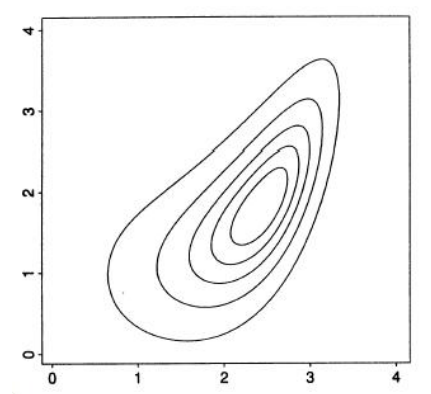

Figura 1.2 - Curvas de nível de duas variáveis aleatórias com distribuição conjunta não elíptica.

Neste sentido, a proposta de Bjerve e Doksum (1993) foi construir uma medida da força da associação local combinando as idéias da regressão não paramétrica (estimação de $E[Y / X=x]$ e de $\operatorname{Var}[Y / X=x]$ ) e de Galton (a força da co-relação entre $X$ e $Y$ pode ser considerada como sendo o coeficiente angular da reta de regressão calculada após $X$ e $Y$ serem padronizadas).

Considere o modelo linear $Y=\alpha+\beta X+\varepsilon$ com $E[\varepsilon]=0, \operatorname{Var}[\varepsilon]=\sigma_{\varepsilon}^{2}$ e $X$ e $\varepsilon$ independentes, e também a correlação linear de Pearson $\rho_{X Y} \equiv \rho$ escrita como

$$
\rho=\beta \sigma_{X} / \sigma_{Y},
$$

em que $\sigma_{X}^{2}$ é a variância de $X$ e $\sigma_{Y}^{2}$ é a variância de $Y$. Utilizando a conhecida decomposição da variância $\operatorname{Var}[Y]=\operatorname{Var}[E[Y / X]]+E[\operatorname{Var}[Y / X]]$, temos que $\operatorname{Var}[E[Y / X]]=\beta^{2} \sigma_{X}^{2}$, que é a variância explicada pela regressão, e $E[\operatorname{Var}[Y / X]]=\sigma_{\varepsilon}^{2}$. Portanto, $\operatorname{Var}[Y]=\sigma_{Y}^{2}=\beta^{2} \sigma_{X}^{2}+\sigma_{\varepsilon}^{2}$, em que $\sigma_{\varepsilon}^{2}=\operatorname{Var}[Y / X=x]$ é a variância residual. Então, a correlação linear de Pearson pode ser reescrita da seguinte forma:

$$
\rho=\frac{\beta \sigma_{X}}{\sqrt{\beta^{2} \sigma_{X}^{2}+\sigma_{\varepsilon}^{2}}},
$$

supondo que $\sigma_{X}^{2}$ e $\sigma_{\mathcal{\varepsilon}}^{2}$ existam. Portanto, esta expressão traduz-se na raiz da variância explicada pela regressão em relação a variabilidade total. Esta representação sugere que no caso de modelos não lineares, uma medida local muito natural da força de associação entre $Y$ e $X$, próximo de $X=x$, é a curva de correlação

$$
\rho(x)=\frac{\beta(x) \sigma_{X}}{\sqrt{\beta^{2}(x) \sigma_{X}^{2}+\sigma_{\varepsilon}^{2}(x)}}, x \in S
$$

em que

$$
\begin{aligned}
& \beta(x)=\frac{\partial}{\partial x} E[Y / X=x], \\
& \sigma_{\mathcal{E}}^{2}(x)=\operatorname{Var}[Y / X=x],
\end{aligned}
$$

supondo que $\sigma_{X}^{2}$ e $\sigma_{\varepsilon}^{2}(x), \forall x \in S$, existam, sendo $S$ o domínio da variável aleatória $X$. 
Este conceito de curva de correlação faz sentido somente quando $X$ é uma variável aleatória contínua pois supomos que $E[Y / X=x]$ seja derivável em $X=x$. Já a distribuição de $Y$ pode ser discreta, contínua ou uma mistura.

Observamos que esta abordagem fornece uma versão padronizada do coeficiente angular de regressão local, sendo portanto, livre de escala.

Para verificarmos o comportamento da curva de correlação local $\rho(x)$, considere a simulação elaborada por Doksum et al. (1994), em que o vetor aleatório $(X, Y)$ tem distribuição normal bivariada com a média e a variância apresentando diferentes valores ao longo do suporte da variável aleatória $X$. Suponha que a variável resposta $Y$ seja relacionada com a covariável $X$ através do modelo

$$
Y=\mu(X)+\sigma(X) \varepsilon,
$$

em que $X$ e $\varepsilon$ são independentes, $X \sim N\left(\mu_{X}, \sigma_{X}^{2}\right), \quad \varepsilon \sim N(0,1)$ e $(X, Y)$ tem densidade conjunta $f(x, y)=f(x) f(y / x)$. Aqui, $f(x)$ é a densidade de $X$ e $f(y / x)$ é $N\left(\mu(x), \sigma^{2}(x)\right)$. Considere $X \sim N\left(1,2 ;(1 / 3)^{2}\right) \quad$ e $\quad Y / X=x \sim N\left(\mu(x), \sigma^{2}(x)\right), \quad$ em que $\mu(x)=(x / 10) \exp \{5-x / 2\}$, $\beta(x)=\mu^{\prime}(x)=(1 / 10)(1-x / 2) \exp (5-x / 2)$ e $\sigma^{2}(x)=\{(1+x / 2) / 3\}^{2}$.

Como resultado, foram obtidos os gráficos da Figura 1.3, sendo que o primeiro representa a densidade conjunta das variáveis aleatórias $X$ e $Y$, e o segundo as suas respectivas curvas de nível, ambos descrevendo uma associação mais forte entre os valores menores das duas variáveis, e uma variabilidade maior dos valores de $Y$ com o aumento dos valores de $X$, indicando heteroscedasticidade. Por fim, no terceiro gráfico desta figura, observamos a curva de correlação decrescendo para zero com o aumento dos valores de $X$.

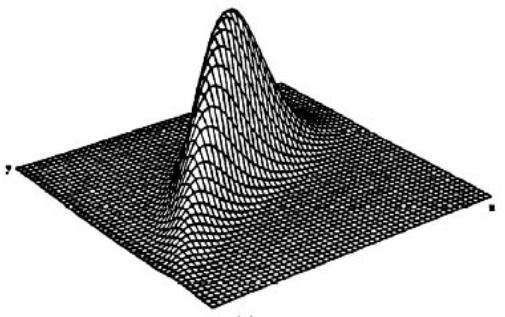

(a)

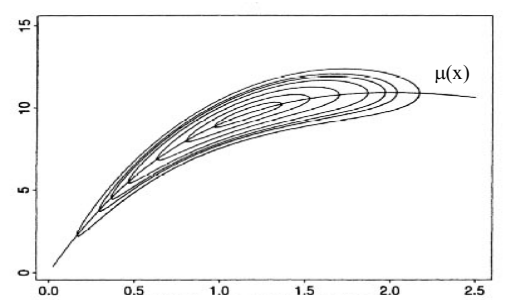

(b)

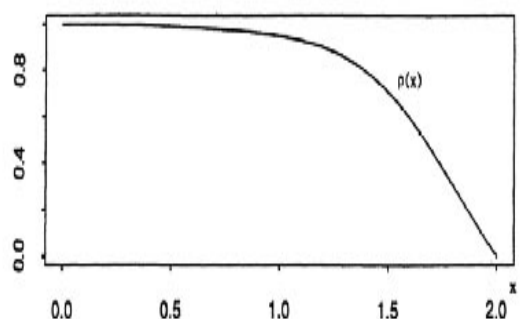

(c)

Figura 1.3 - (a) Densidade conjunta, (b) curvas de nível e (c) curva de correlação de um vetor aleatório bivariado normal tal que $X \sim N\left(1,2 ;(1 / 3)^{2}\right)$ e $Y / X=x \sim N\left(\mu(x), \sigma^{2}(x)\right)$ $\operatorname{com} \mu(x)=(x / 10) \exp \{5-x / 2\}$ e $\sigma^{2}(x)=\{(1+x / 2) / 3\}^{2}$.

Uma crítica que se faz à curva de correlação é que ela é função da variável $X$ somente, não tratando as duas variáveis simetricamente. Além disso, ela não indica a direção da associação localmente.

A curva de correlação satisfaz às seguintes propriedades (veja Bjerve e Doksum, 1993). 
(i) $-1 \leq \rho(x) \leq+1, \forall x \in S$;

(ii) se $X$ e $Y$ são dependentes em regressão ${ }^{(1)}$, então $\rho(x) \geq 0$;

(iii) $\rho(x)$ cresce com o crescimento da dependência em regressão ${ }^{(2)}$;

(iv) se $X$ e $Y$ são independentes, então $\rho(x)=0, \operatorname{com} \sigma_{\varepsilon}^{2}(x)>0, \forall x \in S$;

(v) se $Y=g(X)$, então $\rho(x) \equiv 1, \forall x \in S$ (com $g$ não constante);

(vi) se $\rho(x)= \pm 1$ para quase todo $x \in S$, então $Y=g(x)$;

(vii) se $U=a+b X$ e $V=c+d Y$, com $b d \neq 0$, então

$$
\rho_{U V}(u)=\operatorname{sinal}(\mathrm{bd}) \rho_{X Y}(x), \quad \forall u \in S \text { em que } x=(u-a) / b ;
$$

(viii) se $Y$ é constante, então $\beta(x)=\sigma_{\mathcal{E}}(x)=0$, e $\rho(x)=0$ por definição;

(ix) $\rho_{X Y}(.) \neq \rho_{Y X}($.$) , de modo geral;$

(x) $\rho(x)=\rho$ no caso normal bivariado.

Com referência à estimação da curva de correlação local, Bjerve e Doksum (1993) propõem a estimação via regressão linear local ponderada (Fan, 1993). Já Doksum et al. (1994) estimam a curva de correlação utilizando o estimador empírico vizinho mais próximo e também o estimador kernel de Gasser e Müller (1984).

\subsubsection{Medida de Sibuya}

Com o objetivo de estender o conceito de estatísticas extremas do caso univariado para o caso bivariado, Sibuya (1960) propôs uma função de dependência entre duas variáveis aleatórias, a qual relaciona a distribuição conjunta com as respectivas distribuições marginais.

(1) $(X, Y)$ são dependentes em regressão se $x_{1}<x_{2} \Rightarrow P\left[Y \leq y / X=x_{1}\right]<P\left[Y \leq y / X=x_{2}\right]$. Então, $E[Y / X=x]$ tem derivada positiva, portanto, $\rho(x) \geq 0$.

(2) Sejam $\left(X, Y_{1}\right)$ e $\left(X, Y_{2}\right)$ dois pares de variáveis aleatórias, e considere $Y_{1}(x)=Y_{1} / X=x$ e $Y_{2}(x)=Y_{2} / X=x$ com os respectivos parâmetros de posição e escala $\left(\mu_{1}(x), \sigma_{1}(x)\right)$ e $\left(\mu_{2}(x), \sigma_{2}(x)\right)$. O par $\left(X, Y_{1}\right)$ é dito ser mais dependente em regressão do que o par $\left(X, Y_{2}\right)$, se $Y_{1}(x) / \sigma_{1}(x)$ é estocasticamente mais crescente do que $Y_{2}(x) / \sigma_{2}(x)$, no sentido de que, para cada $\delta$ em alguma vizinhança $(0, \varepsilon)$ do zero, $\left(Y_{1}(x+\delta)-Y_{1}(x-\delta)\right) / \sigma_{1}(x)$ é estocasticamente maior do que $\left(Y_{2}(x+\delta)-Y_{2}(x-\delta)\right) / \sigma_{2}(x)$. E supondo que $\quad \beta_{i}(x)=\partial \mu_{i}(x) / \partial x, \quad i=1,2 \quad$ existam, então segue que $\left(\beta_{1}(x) / \sigma_{1}(x)\right) \geq\left(\beta_{2}(x) / \sigma_{2}(x)\right)$. E como $\rho_{i}(x)$ pode ser reescrita como $\rho_{i}(x)= \pm\left\{1+\left[\sigma_{X} \beta_{i}(x) / \sigma_{i}(x)\right]^{-2}\right\}^{-1 / 2}, i=1,2$, então $\rho_{1}(x) \geq \rho_{2}(x)$, em que $\rho_{i}(x)$ é a curva de correlação de $\left(X, Y_{i}\right), i=1,2$. 
Considere $X$ e $Y$ variáveis aleatórias contínuas definidas num espaço de probabilidades $(\Omega, A, P)$. Suponha que $F(x, y)=P[X \leq x, Y \leq y]$ seja a distribuição conjunta de $X$ e $Y$, e $F_{1}(x)=P[X \leq x]$ e $F_{2}(y)=P[Y \leq y]$ suas respectivas distribuições marginais. Então, a função de dependência de Sibuya, $\Lambda=\Lambda\left(F_{1}(x), F_{2}(y)\right)$, é dada por

$$
\Lambda\left(F_{1}(x), F_{2}(y)\right)=\frac{F(x, y)}{F_{1}(x) F_{2}(y)}, \forall(x, y) \in \mathfrak{R}^{2},
$$

em que $F_{1}(x)>0$ e $F_{2}(y)>0$. Para o caso em que $F_{1}(x)=0$ ou $F_{2}(y)=0$, então $\Lambda(x, y)$ estará definida se existir $\lim _{F_{1}(x) \rightarrow 0} F(x, y) /\left(F_{1}(x) F_{2}(y)\right)$ ou $\lim _{F_{2}(y) \rightarrow 0}\left(F(x, y) / F_{1}(x) F_{2}(y)\right)$, respectivamente.

O comportamento teórico dessa medida de dependência para um vetor aleatório bivariado com distribuição normal padrão e coeficiente de correlação $+0,80,-0,80,+0,20$ e $-0,20$, apresenta-se nos gráficos de perspectiva e de curvas de nível em (a) e (e), (b) e (f), (c) e (g), e (d) e (h), respectivamente, da Figura 1.4. Nestes gráficos, cujas escalas nem sempre são as mesmas, utilizamos grade bivariada com $95 \%$ da distribuição central de cada marginal.

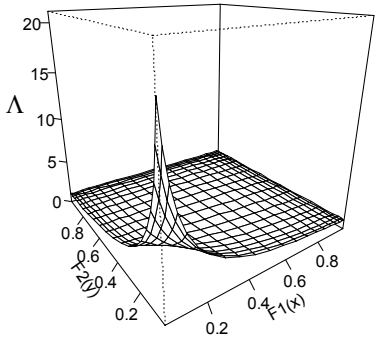

(a)

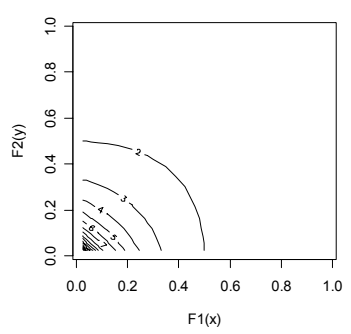

(e)

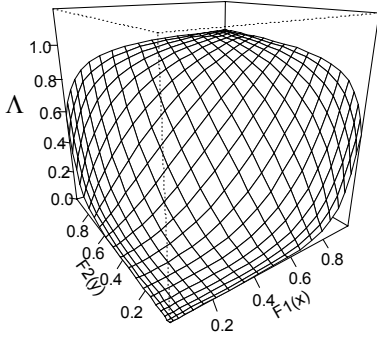

(b)

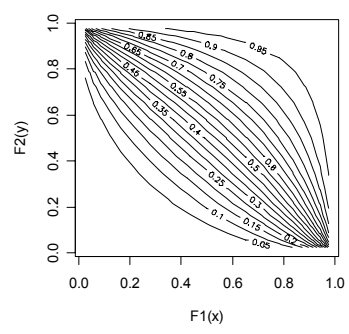

(f)

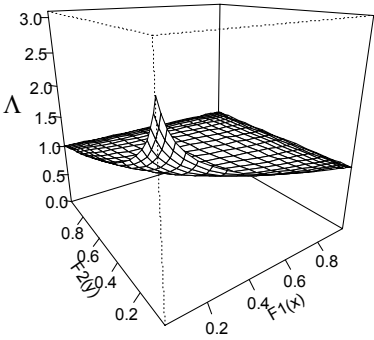

(c)

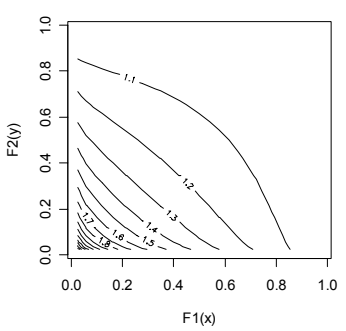

(g)

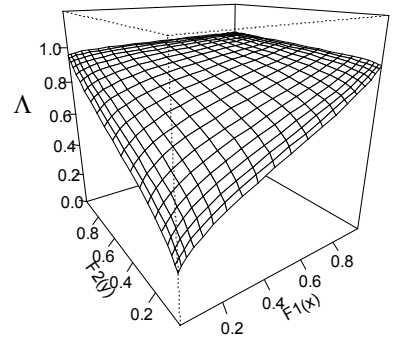

(d)

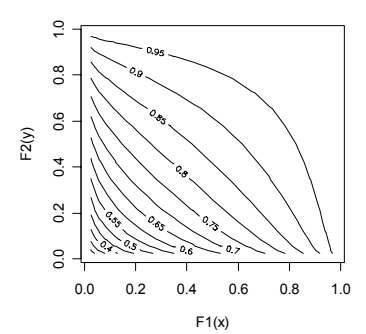

(h)

Figura 1.4 - Gráficos de perspectiva e correspondentes gráficos de curvas de nível da função de dependência local de Sibuya dada por $(1.3)$ para $(X, Y)$ com distribuição normal padrão e coeficiente de correlação $\rho=+0,80$ em (a) e (e), $\rho=-0,80$ em (b) e (f), $\rho=+0,20$ em (c) e (g), e $\rho=-0,20$ em (d) e (h). 
As propriedades para $\Lambda$, definidas por Sibuya (1960), são apresentadas a seguir. Veja também Kolev et al. (2007).

(i) $\quad \operatorname{máx}\left(0, \frac{F_{1}(x)+F_{2}(y)-1}{F_{1}(x) F_{2}(y)}\right) \leq \Lambda\left(F_{1}(x), F_{2}(y)\right) \leq \min \left(\frac{1}{F_{1}(x)}, \frac{1}{F_{2}(y)}\right), \forall(x, y) \in \mathfrak{R}^{2}$;

(ii) $\Lambda\left(F_{1}(x), F_{2}(y)\right)=1, \forall(x, y) \in \mathfrak{R}^{2}$, se e somente se $X$ e $Y$ são independentes;

(iii) Se $X$ e $Y$ são $P Q D(N Q D)^{(3)}$, então $\Lambda\left(F_{1}(X), F_{2}(Y)\right) \geq 1(\leq 1)$ quase certamente;

(iv) Considere as funções arbitrárias $\varphi($.$) e \psi($.$) de X$ e $Y$, respectivamente, tal que $\varphi^{-1}($.$) e \psi^{-1}($.$) existam.$ Utilizando a notação $\bar{F}_{1}(x)=1-F_{1}(x), \bar{F}_{2}(y)=1-F_{2}(y), F_{\varphi(X)}(x)=P[\varphi(X) \leq x], F_{\psi(Y)}(y)=P[\psi(Y) \leq y]$, $\Lambda_{\varphi(X) \psi(Y)}(x, y)=\Lambda\left(F_{\varphi(X)}(x), F_{\psi(Y)}(y)\right), \quad \Lambda_{X Y}\left(\varphi^{-1}(x), \psi^{-1}(y)\right)=\Lambda\left(F_{1}\left(\varphi^{-1}(x)\right), F_{2}\left(\psi^{-1}(y)\right)\right), \quad$ com $\quad S$ sendo o suporte de $(X, Y)$, então:

(a) Se $\varphi($.$) e \psi($.$) são funções crescentes no suporte de X$ e $Y$, respectivamente, então

$$
\Lambda_{\varphi(X) \psi(Y)}(x, y)=\Lambda_{X Y}\left(\varphi^{-1}(x), \psi^{-1}(y)\right), \forall(x, y) \in S ;
$$

(b) Se $\varphi($.$) é uma função decrescente no suporte de X$ e $\psi($.$) é uma função crescente no suporte de Y$, então

$$
\Lambda_{\varphi(X) \psi(Y)}(x, y)=\frac{1}{\bar{F}_{1}\left(\varphi^{-1}(x)\right)}-\frac{F_{1}\left(\varphi^{-1}(x)\right)}{\bar{F}_{1}\left(\varphi^{-1}(x)\right)} \Lambda_{X Y}\left(\varphi^{-1}(x), \psi^{-1}(y)\right), \forall(x, y) \in S
$$

(c) Se $\varphi($.$) é uma função crescente no suporte de X$ e $\psi($.$) é uma função decrescente no suporte de Y$, então

$$
\Lambda_{\varphi(X) \psi(Y)}(x, y)=\frac{1}{\bar{F}_{2}\left(\psi^{-1}(y)\right)}-\frac{F_{2}\left(\psi^{-1}(y)\right)}{\bar{F}_{2}\left(\psi^{-1}(y)\right)} \Lambda_{X Y}\left(\varphi^{-1}(x), \psi^{-1}(y)\right), \forall(x, y) \in S ;
$$

(d) Se $\varphi($.$) e \psi($.$) são funções decrescentes no suporte de X$ e $Y$, respectivamente, então

$$
\Lambda_{\varphi(X) \psi(Y)}(x, y)=\frac{1-F_{1}\left(\varphi^{-1}(x)\right)-F_{2}\left(\psi^{-1}(y)\right)}{\bar{F}_{1}\left(\varphi^{-1}(x)\right) \bar{F}_{2}\left(\psi^{-1}(y)\right)}+\frac{F_{1}\left(\varphi^{-1}(x)\right) F_{2}\left(\psi^{-1}(y)\right)}{\bar{F}_{1}\left(\varphi^{-1}(x)\right) \bar{F}_{2}\left(\psi^{-1}(y)\right)} \Lambda_{X Y}\left(\varphi^{-1}(x), \psi^{-1}(y)\right), \forall(x, y) \in S ;
$$

(v) Se $\rho_{X Y}=0(>1,<1)$ então $\Lambda\left(F_{1}(X), F_{2}(Y)\right)=1(>1,<1)$, no caso normal bivariado.

Pela propriedade (iv), vemos que no ponto $(x, y), \Lambda$ não é ordinalmente invariante sob transformações monótonas pois ela depende das funções $\varphi^{-1}($.$) e \psi^{-1}($.$) , e de possíveis funções das marginais.$

Adicionalmente, Kolev et al. (2007) estabeleceram para $\Lambda$ :

- forma exata da distribuição acumulada de $\Lambda$ para os casos em que $X$ e $Y$ são contramonotônicas, independentes ou comonotônicas, assim como estabeleceram a relação existente entre estas distribuições;

- limite inferior para a esperança de $\Lambda$.

(3) $X$ e $Y$ são $P Q D$ - positively quadrant dependent ( $N Q D$ - negatively quadrant dependent), se para todo $(x, y) \in \mathfrak{R}^{2}$, $F(x, y) \geq(\leq) F_{1}(x) F_{2}(y)$. 
Para estimar a função de dependência de Sibuya $\Lambda$, Kolev et al. (2007) sugerem o estimador empírico $\Lambda_{n}$, o qual, utilizando o método plug-in, é formado através dos estimadores empíricos da distribuição conjunta e das distribuições marginais.

Porém, os estimadores empíricos para as distribuições são altamente descontínuos e algum método de suavização é indicado para se obter melhores resultados. Por exemplo, comparando o estimador empírico para a distribuição com o estimador suavizado via kernel, pode-se verificar em Azzaline (1981) e Hansen (2004) que o erro quadrático médio (EQM) e o EQM integrado assintótico, respectivamente, é menor no estimador suavizado, considerando-se amostras finitas. Então, propomos um estimador suavizado $\hat{\Lambda}$, o qual utilizando o método plug-in, considera os estimadores suavizados via funções kernels das distribuições conjunta e marginais (veja seção 4.1).

Observamos que a função de dependência de Sibuya $\Lambda$ dada por (1.3), também pode ser escrita em termos de cópula.

Cópulas são funções que acoplam funções de distribuição multivariadas com suas respectivas funções de distribuição univariadas. Ou então, cópulas são funções de distribuição multivariadas cujas marginais univariadas são uniformes no intervalo [0,1].Consideremos o caso bivariado a partir de agora. Então, uma cópula $C$ é uma função $C:[0,1]^{2} \rightarrow[0,1]$ que satisfaz determinadas propriedades (Nelsen, 2007).

Pelo teorema de Sklar (1959), sabemos que existe uma cópula $C$ tal que $F(x, y)=C\left(F_{1}(x), F_{2}(y)\right)$, $\forall(x, y) \in \mathfrak{R}^{2}$, sendo que a cópula é única se $F_{1}$ e $F_{2}$ são contínuas. Um corolário imediato deste teorema mostra que $C(u, v)=F\left(F_{1}^{-1}(u), F_{2}^{-1}(v)\right), \quad \forall(u, v) \in[0,1]^{2}, \quad$ em que $F_{1}^{-1}(u)=\inf \left\{x \in \mathfrak{R}: F_{1}(x) \geq u\right\} \equiv \varsigma_{x} \quad$ e $F_{2}^{-1}(v)=\inf \left\{y \in \mathfrak{R}: F_{2}(y) \geq v\right\} \equiv \varsigma_{y}$ são as inversas generalizadas de $F_{1}$ e $F_{2}$, respectivamente. Se $F_{i}$ é estritamente crescente, então a sua inversa generalizada é a inversa, $i=1,2$. Aqui iremos supor que $F_{i}$ são tais que $F_{1}(x)=u$ e $F_{2}(y)=v, u, v \in[0,1]$, admitem soluções únicas dadas por $\varsigma_{x}$ e $\varsigma_{y}$, nesta ordem.

Portanto, reescrevendo (1.3) em termos de cópula, temos

$$
\Lambda(u, v)=\frac{C(u, v)}{u v}, \forall(u, v) \in(0,1]^{2} .
$$

Na Figura 1.5, vemos o comportamento teórico da medida (1.4) novamente para um vetor aleatório bivariado com distribuição normal padrão e correlação $+0,80,-0,80,+0,20$ e $-0,20$, nesta ordem, tanto para os gráficos de perspectiva quanto para os gráficos de curvas de nível. Calculamos esta medida considerando $95 \%$ da região central de cada intervalo $(0,1]$.

Comparando a Figura 1.4 com a Figura 1.5, vemos que $\Lambda$ escrita em termos de funções de distribuição apresenta um comportamento praticamente idêntico ao da medida escrita em termos de cópula. Mais especificamente, a única diferença existente deve-se ao espaçamento dos pontos de grade. Para a função dada por (1.3), as funções de distribuição foram calculadas numa grade $(x, y) \in \mathfrak{R}^{2}$ equiespaçada, e o gráfico de $\Lambda$ foi construído com base na grade $\left(F_{1}(x), F_{2}(y)\right) \in(0,1]^{2}$ não equiespaçada (pois $F_{1}$ e $F_{2}$ não são uniformes). 
Já para a função (1.4), a cópula foi calculada na grade equiespaçada $(u, v) \in(0,1]^{2}$, e $\Lambda$ também. Ou seja, $\Lambda$ calculada através (1.3) e (1.4) resultam em valores idênticos se utilizarmos pontos de grade correspondentes.

As propriedades (i) a (v) de $\Lambda$ escrita como (1.3), continuam válidas para $\Lambda$ dada por (1.4), com as devidas adaptações. Referente à propriedade (iv), observamos para o primeiro caso, por exemplo, que a cópula é invariante sob transformações estritamente crescentes das variáveis aleatórias, mas o mesmo não ocorre com as distribuições marginais. Portanto, a propriedade (iv)-a é válida também para (1.4). De modo similar, pode-se verificar a validade das propriedades (iv)-b a (iv)-d.

Na seção 4.1, propomos o uso de um estimador suavizado via kernel para (1.3) e (1.4), além de descrever o estimador empírico para (1.4).

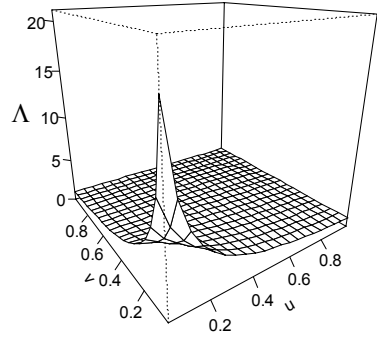

(a)

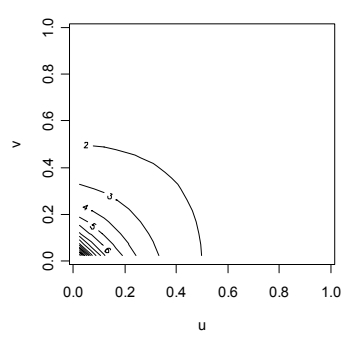

(e)

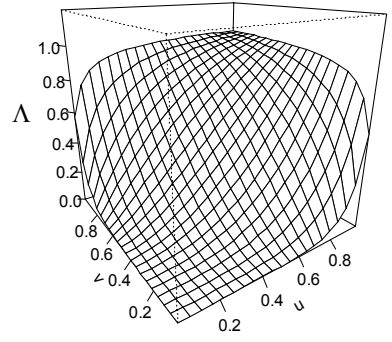

(b)

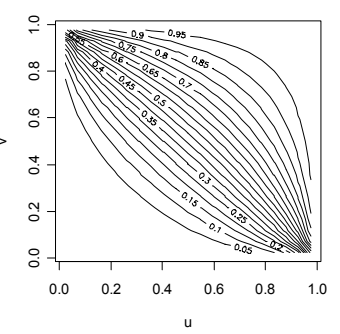

(f)

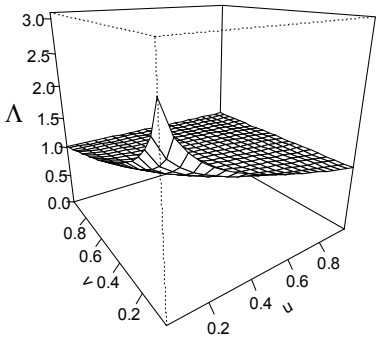

(c)

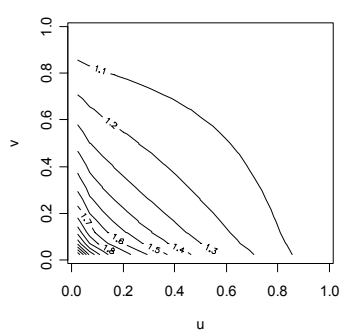

(g)

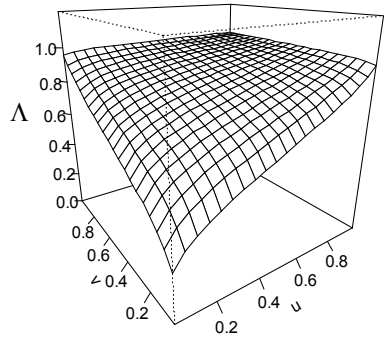

(d)

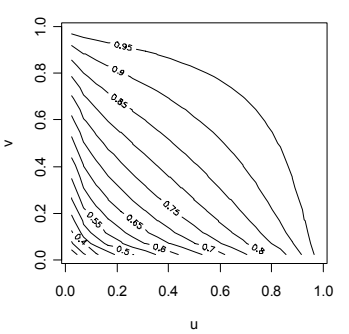

(h)

Figura 1.5 - Gráficos de perspectiva e correspondentes gráficos de curvas de nível da função de dependência local de Sibuya dada por (1.4) para $(X, Y)$ com distribuição normal padrão e coeficiente de correlação $\rho=+0,80$ em (a) e (e), $\rho=-0,80$ em (b) e (f), $\rho=+0,20$ em (c) e (g), e $\rho=-0,20$ em (d) e (h). 


\section{Capítulo 2}

\section{Função de Correlação Local}

\subsection{Função de Autocorrelação Local}

\subsubsection{Introdução}

Seja $\left\{X_{t}, t \in Z\right\}$ um processo estocástico a valores contínuos definido num espaço de probabilidades $(\Omega, A, P)$, e com momento de segunda ordem finito. Aqui, $Z=\{0, \pm 1, \ldots\}$. Considere o vetor aleatório $\left(X_{t_{1}}, X_{t_{2}}\right), t_{1}, t_{2} \in Z$.

Então, a medida de dependência local de Bairamov et al. (2003) dada por (1.1) pode ser definida da seguinte forma:

$$
H\left(x_{1}, x_{2} ; t_{1}, t_{2}\right)=\frac{\rho\left(t_{1}, t_{2}\right)+\varphi_{t_{1}, t_{2}}\left(x_{2}\right) \varphi_{t_{2}, t_{1}}\left(x_{1}\right)}{\sqrt{1+\varphi_{t_{1}, t_{2}}^{2}\left(x_{2}\right)} \sqrt{1+\varphi_{t_{2}, t_{1}}^{2}\left(x_{1}\right)}}, \forall\left(x_{1}, x_{2}\right) \in S, \forall t_{1}, t_{2} \in Z,
$$

em que

$$
\begin{aligned}
& \varphi_{t_{1}, t_{2}}\left(x_{2}\right)=\frac{E\left[X_{t_{1}}\right]-E\left[X_{t_{1}} / X_{t_{2}}=x_{2}\right]}{\sqrt{\operatorname{Var}\left[X_{t_{1}}\right]}}, \\
& \varphi_{t_{2}, t_{1}}\left(x_{1}\right)=\frac{E\left[X_{t_{2}}\right]-E\left[X_{t_{2}} / X_{t_{1}}=x_{1}\right]}{\sqrt{\operatorname{Var}\left[X_{t_{2}}\right]}}, \\
& \rho\left(t_{1}, t_{2}\right)=\frac{E\left[\left(X_{t_{1}}-E\left[X_{t_{1}}\right]\right)\left(X_{t_{2}}-E\left[X_{t_{2}}\right]\right)\right]}{\sqrt{\operatorname{Var}\left[X_{t_{1}}\right]} \sqrt{\operatorname{Var}\left[X_{t_{2}}\right]}} .
\end{aligned}
$$

Suponha agora que o processo $\left\{X_{t}, t \in Z\right\}$ seja estacionário estrito e de segunda ordem, e considere o vetor aleatório $\left(X_{t}, X_{t+\tau}\right), \forall t, \tau \in Z$. Então, a média e a variância são finitas e constantes, e a função de autocorrelação só depende da defasagem. Além disso, todas as distribuições finito dimensionais permanecem as mesmas sob translações do tempo, em particular, as distribuições uni-dimensionais são invariantes sob translações do tempo, portanto $f_{t}(x)=f_{t+\tau}(x), \forall t, \tau \in Z$, o mesmo ocorrendo com as distribuições bidimensionais, $f_{t_{1}, t_{2}}\left(x_{1}, x_{2}\right)=f_{t_{1}+\tau, t_{2}+\tau}\left(x_{1}, x_{2}\right), \forall t_{1}, t_{2} \in Z$. Segue-se que as distribuições bidimensionais dependem de $\left|t_{1}-t_{2}\right|$. Basta fazer, por exemplo, $\tau=-t_{2}$ ou $\tau=-t_{1}$.

Vejamos como se comportam as esperanças condicionais $E\left[X_{t+\tau} / X_{t}=x_{1}\right]$ e $E\left[X_{t} / X_{t+\tau}=x_{2}\right]$. Como $E\left[X_{t}\right]=\mu$ é constante, finita, segue que $X_{t}$ é integrável, $\forall t \in Z$, e então, para todo $x_{1} \in S$ com $f_{t}\left(x_{1}\right)>0$ e para todo $x_{2} \in S$ com $f_{t}\left(x_{2}\right)>0$, temos que estas esperanças condicionais existem e são finitas quase certamente. Portanto, 


$$
\begin{aligned}
& E\left[X_{t+\tau} / X_{t}=x_{1}\right]=\int_{-\infty}^{+\infty} v f_{t+\tau / t}\left(v / x_{1}\right) d v=\frac{1}{f_{t}\left(x_{1}\right)} \int_{-\infty}^{+\infty} v f_{t, t+\tau}\left(x_{1}, v\right) d v, \\
& E\left[X_{t} / X_{t+\tau}=x_{2}\right]=\int_{-\infty}^{+\infty} v f_{t / t+\tau}\left(v / x_{2}\right) d v=\frac{1}{f_{t}\left(x_{2}\right)} \int_{-\infty}^{+\infty} v f_{t, t+\tau}\left(v, x_{2}\right) d v .
\end{aligned}
$$

Para o processo sob estudo, observamos que $\varphi_{t_{1}, t_{2}}\left(x_{2}\right)$ e $\varphi_{t_{2}, t_{1}}\left(x_{1}\right)$ passam a ser escritas como $\varphi_{t, t+\tau}\left(x_{2}\right)$ e $\varphi_{t+\tau, t}\left(x_{1}\right)$, as quais serão denotadas por $\varphi\left(x_{2} ; \tau\right)$ e $\varphi\left(x_{1} ; \tau\right)$, nesta ordem. Para as funções densidade, a partir de agora, consideremos a notação $f_{t}(x)=f(x)$ e $f_{t, t+\tau}\left(x_{1}, x_{2}\right)=f\left(x_{1}, x_{2} ; \tau\right)$. Por fim, sabemos que $\rho\left(t_{1}, t_{2}\right)$ passa a ser representada por $\rho_{\tau}$. Tais considerações são válidas, $\forall t, t_{1}, t_{2}, \tau \in Z$.

Então, a função de autocorrelação local (f.a.c.1.) pode ser escrita como

$$
H_{\tau}\left(x_{1}, x_{2}\right)=\frac{\rho_{\tau}+\varphi\left(x_{2} ; \tau\right) \varphi\left(x_{1} ; \tau\right)}{\sqrt{1+\varphi^{2}\left(x_{2} ; \tau\right)} \sqrt{1+\varphi^{2}\left(x_{1} ; \tau\right)}}, \forall\left(x_{1}, x_{2}\right) \in S, \forall t, \tau \in Z,
$$

em que

$$
\begin{aligned}
& \varphi\left(x_{2} ; \tau\right)=\left(\mu-\frac{1}{f\left(x_{2}\right)} \int_{-\infty}^{+\infty} v f\left(v, x_{2} ; \tau\right) d v\right) / \sigma, \\
& \varphi\left(x_{1} ; \tau\right)=\left(\mu-\frac{1}{f\left(x_{1}\right)} \int_{-\infty}^{+\infty} v f\left(x_{1}, v ; \tau\right) d v\right) / \sigma
\end{aligned}
$$

$\rho_{\tau}$ é a função de autocorrelação da série $X_{t}$.

Portanto, $H_{\tau}\left(x_{1}, x_{2}\right)$ depende de $\tau=\left|t_{1}-t_{2}\right|$, do ponto $\left(x_{1}, x_{2}\right)$, além dos parâmetros $\mu$ e $\sigma$, de $\rho_{\tau}$ e das densidades marginal e conjunta.

Vemos que $H_{\tau}\left(x_{1}, x_{2}\right)$ pode ser representada numa única expressão da seguinte forma

$$
\begin{aligned}
H_{\tau}\left(x_{1}, x_{2}\right)= & \frac{\rho_{\tau}+\left(\left(\mu-E\left[X_{t} / X_{t+\tau}=x_{2}\right]\right) / \sigma\right)\left(\left(\mu-E\left[X_{t+\tau} / X_{t}=x_{1}\right]\right) / \sigma\right)}{\sqrt{1+\left(\left(\mu-E\left[X_{t} / X_{t+\tau}=x_{2}\right]\right) / \sigma\right)^{2}} \sqrt{1+\left(\left(\mu-E\left[X_{t+\tau} / X_{t}=x_{1}\right]\right) / \sigma\right)^{2}}}, \\
& \forall\left(x_{1}, x_{2}\right) \in S, \forall t, \tau \in Z .
\end{aligned}
$$

\subsubsection{Propriedades}

Proposição 2.1. Seja $\left\{X_{t}, t \in Z\right\}$ um processo estocástico a valores contínuos, estacionário estrito e de segunda ordem. Então, a f.a.c.l. $H_{\tau}\left(x_{1}, x_{2}\right)$ satisfaz às seguintes propriedades:

(i) $\left|H_{\tau}\left(x_{1}, x_{2}\right)\right| \leq 1, \forall\left(x_{1}, x_{2}\right) \in S, \forall \tau \in Z$; 
(ii) Se $H_{\tau}\left(x_{1}, x_{2}\right)= \pm 1$ para algum $\left(x_{1}, x_{2}\right) \in S$, então $\rho_{\tau} \neq 0, \forall \tau \in Z$;

(iii) $\mathrm{O}$ ponto $\left(x_{1}^{*}, x_{2}^{*}\right)$ satisfazendo $\varphi\left(x_{1}^{*} ; \tau\right)=\varphi\left(x_{2}^{*} ; \tau\right)=0$ é ponto de sela de $H_{\tau}$, e neste ponto, $H_{\tau}\left(x_{1}^{*}, x_{2}^{*}\right)=\rho_{\tau}, \operatorname{com}\left(x_{1}^{*}, x_{2}^{*}\right) \in S, \forall \tau \in Z ;$

(iv) $H_{-\tau}\left(x_{1}, x_{2}\right)=H_{\tau}\left(x_{1}, x_{2}\right), \forall\left(x_{1}, x_{2}\right) \in S, \forall \tau \in Z$;

(v) $H_{\tau}(\mu, \mu)=\rho_{\tau}, \forall \tau \in Z$, se $\left\{X_{t}\right\}$ é um processo Gaussiano.

As provas dessas propriedades são imediatas.

\subsubsection{Alguns casos especiais}

Nesta seção iremos obter as expressões de $H_{\tau}\left(x_{1}, x_{2}\right)$ para alguns modelos lineares simples.

\subsubsection{Modelo AR(1)}

Considere um processo $\left\{X_{t}, t \in Z\right.$ \} $\}$ estacionário estrito e de segunda ordem, seguindo o modelo $\mathrm{AR}(1)$ representado por $X_{t}=\phi_{0}+\phi_{1} X_{t-1}+a_{t}, a_{t} \sim i i d\left(0, \sigma_{a}^{2}\right)$. Calculemos $H_{\tau}\left(x_{1}, x_{2}\right)$ para este modelo.

Calculemos primeiramente $E\left[X_{t+\tau} / X_{t}=x\right], \forall x \in S, \forall t \in Z$, para $\tau>0$. Por exemplo, se $\tau=1$, então:

$$
\begin{aligned}
E\left[X_{t+1} / X_{t}=x\right] & =E\left[\phi_{0}+\phi_{1} X_{t}+a_{t+1} / X_{t}=x\right]=\phi_{0}+\phi_{1} x+E\left[a_{t+1} / X_{t}=x\right] \\
& =\phi_{0}+\phi_{1} x,
\end{aligned}
$$

pois $X_{t}$ depende de $a_{t-j}, j \geq 0$.

De modo geral,

$$
E\left[X_{t+\tau} / X_{t}=x\right]=\sum_{k=0}^{\tau-1} \phi_{0} \phi_{1}^{k}+\phi_{1}^{\tau} x, \tau \geq 1,
$$

ou seja

$$
E\left[X_{t+\tau} / X_{t}=x\right]=\frac{\phi_{0}}{1-\phi_{1}}+\phi_{1}^{\tau}\left(x-\frac{\phi_{0}}{1-\phi_{1}}\right), \tau \geq 1 .
$$

Além disso, podemos verificar que $E\left[X_{t+\tau} / X_{t}=x\right]=\frac{\phi_{0}}{1-\phi_{1}}+\phi_{1}^{-\tau}\left(x-\frac{\phi_{0}}{1-\phi_{1}}\right), \tau \leq-1$, portanto,

$$
E\left[X_{t+\tau} / X_{t}=x\right]=\frac{\phi_{0}}{1-\phi_{1}}+\phi_{1}^{|\tau|}\left(x-\frac{\phi_{0}}{1-\phi_{1}}\right),|\tau| \geq 1 \text {. }
$$

Agora, para calcularmos $E\left[X_{t} / X_{t+\tau}=x\right], \forall x \in S, \forall t \in Z, \forall \tau>0$, vamos utilizar o processo backward (Box e Jenkins, 1976). Sabemos que a forma usual de representar um modelo AR(1) é $X_{t}=\phi_{0}+\phi_{1} X_{t-1}+a_{t}$, $a_{t} \sim\left(0, \sigma_{a}^{2}\right)$ independentes, ou $\Phi(B) \tilde{X}_{t}=a_{t}$, em que $\tilde{X}_{t}=X_{t}-\mu$ e $\Phi(B)=1-\phi_{1} B$, que reescrita como 
um processo backward resulta em $\Phi(F) \widetilde{X}_{t}=e_{t}, e_{t} \sim\left(0, \sigma_{a}^{2}\right)$ independentes, em que $\Phi(F)=1-\phi_{1} F$. Portanto, um modelo AR(1) escrito como um processo backward pode ser representado por

$$
X_{t}=\phi_{0}+\phi_{1} X_{t+1}+e_{t}, e_{t} \sim\left(0, \sigma_{a}^{2}\right) \text { independentes. }
$$

Então, para $\tau=1$,

$$
\begin{aligned}
E\left[X_{t} / X_{t+1}=x\right] & =E\left[\phi_{0}+\phi_{1} X_{t+1}+e_{t} / X_{t+1}=x\right]=\phi_{0}+\phi_{1} x+E\left[e_{t} / X_{t+1}=x\right] \\
& =\phi_{0}+\phi_{1} x,
\end{aligned}
$$

pois $X_{t}$ depende de $e_{t+j}, j \geq 0$.

De modo geral, temos

$$
E\left[X_{t} / X_{t+\tau}=x\right]=\sum_{k=0}^{\tau-1} \phi_{0} \phi_{1}^{k}+\phi_{1}^{\tau} x, \tau \geq 1,
$$

ou então

$$
E\left[X_{t} / X_{t+\tau}=x\right]=\frac{\phi_{0}}{1-\phi_{1}}+\phi_{1}^{\tau}\left(x-\frac{\phi_{0}}{1-\phi_{1}}\right), \tau \geq 1 .
$$

Para $\tau \leq-1$, temos que $E\left[X_{t} / X_{t+\tau}=x\right]=\frac{\phi_{0}}{1-\phi_{1}}+\phi_{1}^{-\tau}\left(x-\frac{\phi_{0}}{1-\phi_{1}}\right)$, e então

$$
E\left[X_{t} / X_{t+\tau}=x\right]=\frac{\phi_{0}}{1-\phi_{1}}+\phi_{1}^{|\tau|}\left(x-\frac{\phi_{0}}{1-\phi_{1}}\right),|\tau| \geq 1 .
$$

Podemos observar que as expressões de $E\left[X_{t+\tau} / X_{t}=x\right]$ e $E\left[X_{t} / X_{t+\tau}=x\right]$ têm formas idênticas.

Passemos ao cálculo de $H_{\tau}\left(x_{1}, x_{2}\right)$.

Sabemos que para o modelo dado acima,

$$
\mu=\frac{\phi_{0}}{1-\phi_{1}}, \quad \sigma^{2}=\frac{\sigma_{a}^{2}}{1-\phi_{1}^{2}}, \quad \rho_{\tau}=\left\{\begin{array}{c}
1, \tau=0 \\
\phi_{1}^{|\tau|},|\tau| \geq 1
\end{array} \text { sendo } \rho_{-\tau}=\rho_{\tau} .\right.
$$

Então, substituindo estes termos na expressão geral de $H_{\tau}\left(x_{1}, x_{2}\right)$ dada por (2.1), temos que para $|\tau| \geq 1$,

$$
H_{\tau}\left(x_{1}, x_{2}\right)=\frac{\phi_{1}^{|\tau|}+\phi_{1}^{2|\tau|}\left(\frac{x_{2}-\phi_{0} /\left(1-\phi_{1}\right)}{\sigma_{a} / \sqrt{1-\phi_{1}^{2}}}\right)\left(\frac{x_{1}-\phi_{0} /\left(1-\phi_{1}\right)}{\sigma_{a} / \sqrt{1-\phi_{1}^{2}}}\right)}{\sqrt{1+\phi_{1}^{2|\tau|}\left(\frac{x_{2}-\phi_{0} /\left(1-\phi_{1}\right)}{\sigma_{a} / \sqrt{1-\phi_{1}^{2}}}\right)^{2}} \sqrt{1+\phi_{1}^{2|\tau|}\left(\frac{x_{1}-\phi_{0} /\left(1-\phi_{1}\right)}{\sigma_{a} / \sqrt{1-\phi_{1}^{2}}}\right)^{2}}},
$$

ou

$$
\begin{aligned}
H_{\tau}\left(x_{1}, x_{2}\right) & =\frac{\phi_{1}^{|\tau|} \sigma_{a}^{2}\left(1-\phi_{1}\right)^{2}+\phi_{1}^{2|\tau|}\left(1-\phi_{1}^{2}\right)\left(\left(1-\phi_{1}\right) x_{2}-\phi_{0}\right)\left(\left(1-\phi_{1}\right) x_{1}-\phi_{0}\right)}{\left[\left(\sigma_{a}^{2}\left(1-\phi_{1}\right)^{2}+\phi_{1}^{2|\tau|}\left(1-\phi_{1}^{2}\right)\left\{\left(1-\phi_{1}\right) x_{2}-\phi_{0}\right\}^{2}\right)\left(\sigma_{a}^{2}\left(1-\phi_{1}\right)^{2}+\phi_{1}^{2|\tau|}\left(1-\phi_{1}^{2}\right)\left\{\left(1-\phi_{1}\right) x_{1}-\phi_{0}\right\}^{2}\right)\right]^{1 / 2}} . \\
& |\tau| \geq 1,
\end{aligned}
$$


Através da simulação desta função, cujos gráficos encontram-se na Figura 2.1 (seção 2.6), em que consideramos $\phi_{0}=0, \phi_{1}=+0,80$ e $\sigma_{a}^{2}=1$, podemos observar a presença de um decaimento exponencial para zero a partir do ponto de sela. Este é um comportamento similar à f.a.c. de um modelo AR(1). Veja a seção 2.6 para a descrição detalhada das simulações.

\subsubsection{Modelo MA(1)}

Considere um processo $\left\{X_{t}, t \in Z\right\}$ estacionário estrito e de segunda ordem, seguindo o modelo MA(1) representado por $X_{t}=\theta_{0}-\theta_{1} a_{t-1}+a_{t}, a_{t} \sim i i d\left(0, \sigma_{a}^{2}\right)$. Calculemos $H_{\tau}\left(x_{1}, x_{2}\right)$ para este modelo.

Primeiramente, vamos calcular $E\left[X_{t+\tau} / X_{t}=x\right], \forall x \in S, \forall t \in Z$, para $\tau>0$. Para tanto, vamos reescrever este modelo em termos das observações passadas da série temporal, ou seja, como um modelo $\operatorname{AR}(\infty)$, isto é

$$
X_{t}=\theta_{0} /\left(1-\theta_{1}\right)-\sum_{j=1}^{\infty} \theta_{1}^{j} X_{t-j}+a_{t}, a_{t} \sim \operatorname{iid}\left(0, \sigma_{a}^{2}\right) .
$$

Então, temos

- $\tau=1$

$$
\begin{aligned}
E\left[X_{t+1} / X_{t}=x\right]=E\left[\theta_{0} /\left(1-\theta_{1}\right)-\theta_{1} X_{t}-\theta_{1}^{2} X_{t-1}-\theta_{1}^{3} X_{t-2}-\ldots+a_{t+1} / X_{t}=x\right] \\
\quad=\theta_{0} /\left(1-\theta_{1}\right)-\theta_{1} x-\theta_{1}^{2} E\left[X_{t-1} / X_{t}=x\right]-\theta_{1}^{3} E\left[X_{t-2} / X_{t}=x\right]-\ldots+E\left[a_{t+1} / X_{t}=x\right] \\
\quad=\theta_{0} /\left(1-\theta_{1}\right)-\theta_{1} x-\theta_{1}^{2} E\left[X_{t-1}\right]-\theta_{1}^{3} E\left[X_{t-2}\right]-\ldots+E\left[a_{t+1}\right]=\theta_{0} /\left(1-\theta_{1}\right)-\theta_{1} x-\theta_{0} \theta_{1}^{2}-\theta_{0} \theta_{1}^{3}-\ldots \\
\quad=\theta_{0} /\left(1-\theta_{1}\right)-\theta_{1} x-\theta_{0} \theta_{1}^{2} /\left(1-\theta_{1}\right)
\end{aligned}
$$

pois $X_{t-j}, j \geq 1$ não depende de $X_{t}$, e $X_{t}$ depende de $a_{t-j}, j \geq 0$;

- $\tau=2$

$$
\begin{aligned}
E\left[X_{t+2} /\right. & \left.X_{t}=x\right]=E\left[\theta_{0} /\left(1-\theta_{1}\right)-\theta_{1} X_{t+1}-\theta_{1}^{2} X_{t}-\theta_{1}^{3} X_{t-1}-\theta_{1}^{4} X_{t-2}-\ldots+a_{t+2} / X_{t}=x\right] \\
& =\theta_{0} /\left(1-\theta_{1}\right)-\theta_{1} E\left[X_{t+1} / X_{t}=x\right]-\theta_{1}^{2} x-\theta_{1}^{3} E\left[X_{t-1} / X_{t}=x\right]-\ldots+E\left[a_{t+2} / X_{t}=x\right] \\
& =\theta_{0} /\left(1-\theta_{1}\right)-\theta_{1}\left\{\theta_{0} /\left(1-\theta_{1}\right)-\theta_{1} x-\theta_{0} \theta_{1}^{2} /\left(1-\theta_{1}\right)\right\}-\theta_{1}^{2} x-\theta_{1}^{3} E\left[X_{t-1}\right]-\ldots+E\left[a_{t+2}\right] \\
& =\theta_{0} /\left(1-\theta_{1}\right)-\theta_{0} \theta_{1} /\left(1-\theta_{1}\right)+\theta_{0} \theta_{1}^{3} /\left(1-\theta_{1}\right)+\theta_{1}^{2} x-\theta_{1}^{2} x-\theta_{0} \theta_{1}^{3}-\theta_{0} \theta_{1}^{4}-\ldots \\
& =\theta_{0} /\left(1-\theta_{1}\right)-\theta_{0} \theta_{1} /\left(1-\theta_{1}\right)+\theta_{0} \theta_{1}^{3} /\left(1-\theta_{1}\right)-\theta_{0} \theta_{1}^{3} /\left(1-\theta_{1}\right)=\theta_{0}\left(1-\theta_{1}\right) /\left(1-\theta_{1}\right) \\
& =\theta_{0} .
\end{aligned}
$$

De modo geral, $\forall x \in S, \forall t \in Z$ e $\tau>0$, temos

$$
E\left[X_{t+\tau} / X_{t}=x\right]=\left\{\begin{array}{ll}
\frac{\theta_{0}}{1-\theta_{1}}-\theta_{1}\left(x+\frac{\theta_{0} \theta_{1}}{1-\theta_{1}}\right) & , \tau=1 \\
\theta_{0} & , \tau \geq 2
\end{array} .\right.
$$

Também verificamos que esta mesma expressão é obtida para $\tau<0$, portanto, 


$$
E\left[X_{t+\tau} / X_{t}=x\right]= \begin{cases}\frac{\theta_{0}}{1-\theta_{1}}-\theta_{1}\left(x+\frac{\theta_{0} \theta_{1}}{1-\theta_{1}}\right) & ,|\tau|=1 \\ \theta_{0} & ,|\tau| \geq 2\end{cases}
$$

Agora, para calcularmos $E\left[X_{t} / X_{t+\tau}=x\right], \forall x \in S, \forall t \in Z$ para $\tau>0$, vamos novamente utilizar o processo backward, escrevendo então o modelo MA(1) nesta forma. Um modelo MA(1) escrito como um processo backward pode representado por

$$
X_{t}=\theta_{0}-\theta_{1} e_{t+1}+e_{t}, e_{t} \sim\left(0, \sigma_{a}^{2}\right) \text { independentes. }
$$

Agora, vamos reescrever este modelo em termos das observações futuras da série temporal, ou seja, como um modelo $\operatorname{AR}(\infty)$, isto é

$$
X_{t}=\theta_{0} /\left(1-\theta_{1}\right)-\sum_{j=1}^{\infty} \theta_{1}^{j} X_{t+j}+e_{t}, e_{t} \sim \operatorname{iid}\left(0, \sigma_{a}^{2}\right) .
$$

Então, temos

- $\tau=1$

$$
\begin{aligned}
E\left[X_{t} / X_{t+1}=x\right]=E\left[\theta_{0} /\left(1-\theta_{1}\right)-\theta_{1} X_{t+1}-\theta_{1}^{2} X_{t+2}-\theta_{1}^{3} X_{t+3}-\ldots+e_{t} / X_{t+1}=x\right] \\
\quad=\theta_{0} /\left(1-\theta_{1}\right)-\theta_{1} x-\theta_{1}^{2} E\left[X_{t+2} / X_{t+1}=x\right]-\theta_{1}^{3} E\left[X_{t+3} / X_{t+1}=x\right]-\ldots+E\left[e_{t} / X_{t+1}=x\right] \\
\quad=\theta_{0} /\left(1-\theta_{1}\right)-\theta_{1} x-\theta_{1}^{2} E\left[X_{t+2}\right]-\theta_{1}^{3} E\left[X_{t+3}\right]-\ldots+E\left[e_{t}\right]=\theta_{0} /\left(1-\theta_{1}\right)-\theta_{1} x-\theta_{0} \theta_{1}^{2}-\theta_{0} \theta_{1}^{3}-\ldots \\
\quad=\theta_{0} /\left(1-\theta_{1}\right)-\theta_{1} x-\theta_{0} \theta_{1}^{2} /\left(1-\theta_{1}\right),
\end{aligned}
$$

pois $X_{t+j}, j \geq 1$ não depende de $X_{t}$, e $X_{t}$ depende de $e_{t+j}, j \geq 0$;

- $\tau=2$

$$
\begin{aligned}
E[ & \left.X_{t} / X_{t+2}=x\right]=E\left[\theta_{0} /\left(1-\theta_{1}\right)-\theta_{1} X_{t+1}-\theta_{1}^{2} X_{t+2}-\theta_{1}^{3} X_{t+3}-\theta_{1}^{4} X_{t+4}-\ldots+e_{t} / X_{t+2}=x\right] \\
& =\theta_{0} /\left(1-\theta_{1}\right)-\theta_{1} E\left[X_{t+1} / X_{t+2}=x\right]-\theta_{1}^{2} x-\theta_{1}^{3} E\left[X_{t+3} / X_{t+2}=x\right]-\ldots+E\left[e_{t} / X_{t+2}=x\right] \\
& =\theta_{0} /\left(1-\theta_{1}\right)-\theta_{1}\left\{\theta_{0} /\left(1-\theta_{1}\right)-\theta_{1} x-\theta_{0} \theta_{1}^{2} /\left(1-\theta_{1}\right)\right\}-\theta_{1}^{2} x-\theta_{1}^{3} E\left[X_{t+3}\right]-\theta_{1}^{4} E\left[X_{t+4}\right]-\ldots+E\left[e_{t}\right] \\
& =\theta_{0} /\left(1-\theta_{1}\right)-\theta_{0} \theta_{1} /\left(1-\theta_{1}\right)+\theta_{0} \theta_{1}^{3} /\left(1-\theta_{1}\right)+\theta_{1}^{2} x-\theta_{1}^{2} x-\theta_{0} \theta_{1}^{3}-\theta_{0} \theta_{1}^{4}-\ldots \\
& =\theta_{0} /\left(1-\theta_{1}\right)-\theta_{0} \theta_{1} /\left(1-\theta_{1}\right)+\theta_{0} \theta_{1}^{3} /\left(1-\theta_{1}\right)-\theta_{0} \theta_{1}^{3} /\left(1-\theta_{1}\right)=\theta_{0}\left(1-\theta_{1}\right) /\left(1-\theta_{1}\right) \\
& =\theta_{0} .
\end{aligned}
$$

De modo geral, $\forall x \in S, \forall t \in Z$ e $\tau>0$, temos

$$
E\left[X_{t} / X_{t+\tau}=x\right]=\left\{\begin{array}{ll}
\frac{\theta_{0}}{1-\theta_{1}}-\theta_{1}\left(x+\frac{\theta_{0} \theta_{1}}{1-\theta_{1}}\right) & , \tau=1 \\
\theta_{0} & , \tau \geq 2
\end{array},\right.
$$

sendo que o mesmo resultado é obtido para $\tau<0$. Então, 


$$
E\left[X_{t} / X_{t+\tau}=x\right]=\left\{\begin{array}{ll}
\frac{\theta_{0}}{1-\theta_{1}}-\theta_{1}\left(x-\frac{\theta_{0} \theta_{1}}{1-\theta_{1}}\right) & ,|\tau|=1 \\
\theta_{0} & ,|\tau| \geq 2
\end{array} .\right.
$$

Podemos observar que $E\left[X_{t+\tau} / X_{t}=x\right]$ e $E\left[X_{t} / X_{t+\tau}=x\right]$ têm formas idênticas.

Passemos ao cálculo de $H_{\tau}\left(x_{1}, x_{2}\right)$. Sabemos que para o modelo MA(1) dado anteriormente,

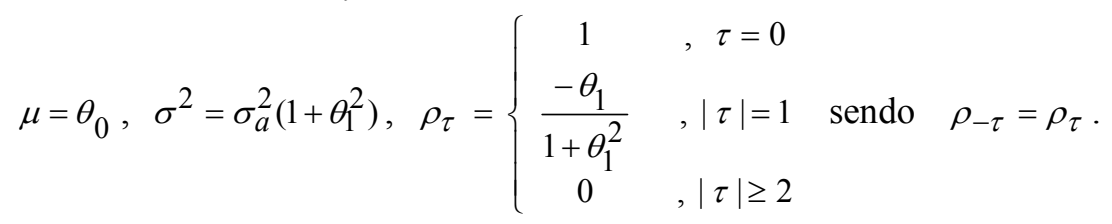

Então, substituindo estes termos na expressão geral de $H_{\tau}\left(x_{1}, x_{2}\right)$ dada por (2.1), temos que para $|\tau| \geq 1$,

$$
H_{\tau}\left(x_{1}, x_{2}\right)= \begin{cases}\frac{\frac{-\theta_{1}}{1+\theta_{1}^{2}}+\left(\left(\theta_{1} x_{2}-\theta_{0} \theta_{1}\right) / \sigma_{a} \sqrt{1+\theta_{1}^{2}}\right)\left(\left(\theta_{1} x_{1}-\theta_{0} \theta_{1}\right) / \sigma_{a} \sqrt{1+\theta_{1}^{2}}\right)}{\sqrt{1+\left(\left(\theta_{1} x_{2}-\theta_{0} \theta_{1}\right) / \sigma_{a} \sqrt{1+\theta_{1}^{2}}\right)^{2}} \sqrt{1+\left(\left(\theta_{1} x_{1}-\theta_{0} \theta_{1}\right) / \sigma_{a} \sqrt{1+\theta_{1}^{2}}\right)^{2}}} & ,|\tau|=1 \\ 0 & ,|\tau| \geq 2\end{cases}
$$

$\mathrm{ou}$

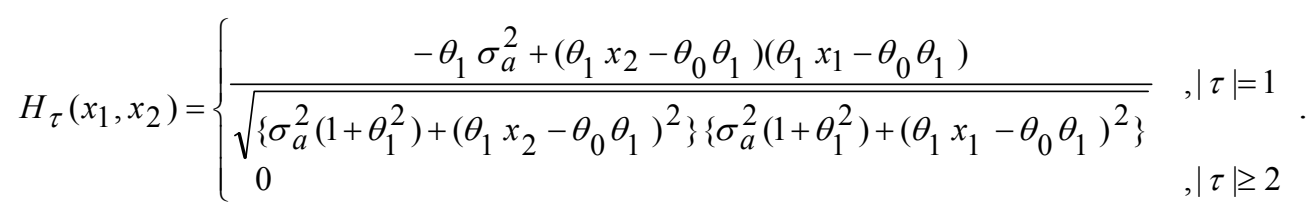

Simulando esta função com $\theta_{0}=0, \theta_{1}=-0,80$ e $\sigma_{a}^{2}=1$ (veja os gráficos da Figura 2.7, seção 2.6), podemos observar a presença da função não nula somente no $\operatorname{lag} 1$, assim como ocorre com a f.a.c. do MA(1). Mais informações sobre as simulações podem ser obtidas na seção 2.6.

\subsubsection{Modelo ARMA $(1,1)$}

Considere um processo $\left\{X_{t}, t \in Z\right\}$ estacionário estrito e de segunda ordem, seguindo o modelo $\operatorname{ARMA}(1,1)$ com média zero, representado por $X_{t}=\phi X_{t-1}-\theta a_{t-1}+a_{t}, \quad a_{t} \sim \operatorname{iid}\left(0, \sigma_{a}^{2}\right)$. Calculemos $H_{\tau}\left(x_{1}, x_{2}\right)$ para este modelo.

Inicialmente, vamos calcular $E\left[X_{t+\tau} / X_{t}=x\right], \forall x \in S, \forall t \in \mathcal{Z}$, para $\tau>0$. Para tanto, vamos reescrever este modelo em termos somente das observações passadas da série temporal, ou seja, como um modelo $\operatorname{AR}(\infty)$, isto é

$$
X_{t}=\sum_{j=1}^{\infty} \theta^{j-1}(\phi-\theta) X_{t-j}+a_{t}, a_{t} \sim \operatorname{iid}\left(0, \sigma_{a}^{2}\right) .
$$

Então temos:

- $\tau=1$

$$
E\left[X_{t+1} / X_{t}=x\right]=E\left[(\phi-\theta) X_{t}+\theta(\phi-\theta) X_{t-1}+\theta^{2}(\phi-\theta) X_{t-2}+\ldots+a_{t+1} / X_{t}=x\right]
$$




$$
\begin{aligned}
& =(\phi-\theta) x+\theta(\phi-\theta) E\left[X_{t-1} / X_{t}=x\right]+\theta^{2}(\phi-\theta) E\left[X_{t-2} / X_{t}=x\right]+\ldots+E\left[a_{t+1} / X_{t}=x\right] \\
& =(\phi-\theta) x,
\end{aligned}
$$

pois $X_{t}$ depende de $X_{t-j}, j \geq 1$ e $X_{t}$ depende de $a_{t-j}, j \geq 0$;

- $\tau=2$

$$
\begin{aligned}
E\left[X_{t+2} / X_{t}=x\right] & =E\left[(\phi-\theta) X_{t+1}+\theta(\phi-\theta) X_{t}+\theta^{2}(\phi-\theta) X_{t-1}+\ldots+a_{t+2} / X_{t}=x\right] \\
& =(\phi-\theta) E\left[X_{t+1} / X_{t}=x\right]+\theta(\phi-\theta) x+\theta^{2}(\phi-\theta) E\left[X_{t-1} / X_{t}=x\right]+\ldots+E\left[a_{t+2} / X_{t}=x\right] \\
& =(\phi-\theta)^{2} x+\theta(\phi-\theta) x=\phi(\phi-\theta) x .
\end{aligned}
$$

De modo geral, $\forall x \in S, \forall t \in Z$ e $\tau>0$, temos

$$
E\left[X_{t+\tau} / X_{t}=x\right]=\phi^{\tau-1}(\phi-\theta) x, \tau \geq 1,
$$

e para $\tau<0$, é fácil ver que $E\left[X_{t+\tau} / X_{t}=x\right]=\phi^{-\tau-1}(\phi-\theta) x$. Portanto,

$$
E\left[X_{t+\tau} / X_{t}=x\right]=\phi^{|\tau|-1}(\phi-\theta) x,|\tau| \geq 1 \text {. }
$$

Agora, para calcularmos $E\left[X_{t} / X_{t+\tau}=x\right], \forall x \in S, \forall t \in Z$ para $\tau>0$, vamos utilizar mais uma vez o processo backward, escrevendo então o modelo $\operatorname{ARMA}(1,1)$ nesta forma. Um modelo $\operatorname{ARMA}(1,1)$ escrito como um processo backward pode ser representado por

$$
X_{t}=\phi X_{t+1}-\theta e_{t+1}+e_{t}, \quad e_{t} \sim\left(0, \sigma_{a}^{2}\right) \text { independentes. }
$$

Agora, vamos reescrever este modelo em termos somente das observações futuras da série temporal, ou seja, como um modelo $\mathrm{AR}(\infty)$, isto é

Então temos

$$
X_{t}=\sum_{j=1}^{\infty} \theta^{j-1}(\phi-\theta) X_{t+j}+e_{t}, e_{t} \sim \operatorname{iid}\left(0, \sigma_{a}^{2}\right) .
$$

- $\tau=1$

$$
\begin{aligned}
E\left[X_{t} / X_{t+1}=x\right] & =E\left[(\phi-\theta) X_{t+1}+\theta(\phi-\theta) X_{t+2}+\theta^{2}(\phi-\theta) X_{t+3}+\ldots+e_{t} / X_{t+1}=x\right] \\
& =(\phi-\theta) x+\theta(\phi-\theta) E\left[X_{t+2} / X_{t+1}=x\right]+\theta^{2}(\phi-\theta) E\left[X_{t+3} / X_{t+1}=x\right]+\ldots+E\left[e_{t} / X_{t+1}=x\right] \\
& =(\phi-\theta) x,
\end{aligned}
$$

pois $X_{t}$ depende de $X_{t+j}, j \geq 1$ e $X_{t}$ depende de $e_{t+j}, j \geq 0$;

- $\tau=2$

$$
\begin{aligned}
E\left[X_{t} / X_{t+2}=x\right] & =E\left[(\phi-\theta) X_{t+1}+\theta(\phi-\theta) X_{t+2}+\theta^{2}(\phi-\theta) X_{t+3}+\ldots+e_{t} / X_{t+2}=x\right] \\
& =(\phi-\theta) E\left[X_{t+1} / X_{t+2}=x\right]+\theta(\phi-\theta) x+\theta^{2}(\phi-\theta) E\left[X_{t+3} / X_{t+2}=x\right]+\ldots+E\left[e_{t} / X_{t+2}=x\right] \\
& =(\phi-\theta)^{2} x+\theta(\phi-\theta) x=\phi(\phi-\theta) x .
\end{aligned}
$$

De modo geral, $\forall x \in S, \forall t \in Z$ e $\tau>0$, temos

$$
E\left[X_{t} / X_{t+\tau}=x\right]=\phi^{\tau-1}(\phi-\theta) x, \tau \geq 1 \text {. }
$$

Já para $\tau<0$, obtivemos $E\left[X_{t} / X_{t+\tau}=x\right]=\phi^{-\tau-1}(\phi-\theta) x$. Então

$$
E\left[X_{t} / X_{t+\tau}=x\right]=\phi^{|\tau|-1}(\phi-\theta) x,|\tau| \geq 1 .
$$


Novamente neste modelo, $E\left[X_{t+\tau} / X_{t}=x\right]$ e $E\left[X_{t} / X_{t+\tau}=x\right]$ têm formas idênticas.

Agora, para o modelo ARMA(1,1) considerado, temos

$$
\mu=0, \sigma^{2}=\frac{1+\theta^{2}-2 \phi \theta}{1-\phi^{2}} \sigma_{a}^{2}, \quad \rho_{\tau}=\left\{\begin{array}{cl}
1 & , \tau=0 \\
\phi^{\tau-1} \frac{(1-\phi \theta)(\phi-\theta)}{1+\theta^{2}-2 \phi \theta} & ,|\tau| \geq 1
\end{array} \text { sendo } \rho_{-\tau}=\rho_{\tau} .\right.
$$

Então, substituindo estes termos na expressão geral de $H_{\tau}\left(x_{1}, x_{2}\right)$ dada por (2.1), temos que para $|\tau| \geq 1$,

$$
H_{\tau}\left(x_{1}, x_{2}\right)=\frac{\frac{\phi_{1}^{|\tau|-1}(1-\phi \theta)(\phi-\theta)}{1+\theta^{2}-2 \phi \theta}+\frac{\left(\phi^{|\tau|-1}(\phi-\theta) x_{2}\right)}{\left(\sigma_{a} \sqrt{\frac{1+\theta^{2}-2 \phi \theta}{1-\phi^{2}}}\right) \frac{\left(\phi^{|\tau|-1}(\phi-\theta) x_{1}\right)}{\left(\sigma_{a} \sqrt{\frac{1+\theta^{2}-2 \phi \theta}{1-\phi^{2}}}\right)}}}{\sqrt{\left(\sigma_{a} \sqrt{\frac{1+\theta^{2}-2 \phi \theta}{1-\phi^{2}}}\right)^{2}} \sqrt{\left(\phi_{a} \sqrt{\frac{1+\theta^{2}-2 \phi \theta}{1-\phi^{2}}}\right)^{2}}}
$$

ou

$$
\begin{aligned}
H_{\tau}\left(x_{1}, x_{2}\right) & =\frac{\sigma_{a}^{2} \phi^{|\tau|-1}(1-\phi \theta)(\phi-\theta)+x_{1} x_{2} \phi^{2(|\tau|-1)}(\phi-\theta)^{2}\left(1-\phi^{2}\right)}{\sqrt{\left(\sigma_{a}^{2}\left(1+\theta^{2}-2 \phi \theta\right)+\phi^{2(|\tau|-1)}(\phi-\theta)^{2}\left(1-\phi^{2}\right) x_{1}^{2}\right)\left(\sigma_{a}^{2}\left(1+\theta^{2}-2 \phi \theta\right)+\phi^{2(|\tau|-1)}(\phi-\theta)^{2}\left(1-\phi^{2}\right) x_{2}^{2}\right)}}, \\
& |\tau| \geq 1 .
\end{aligned}
$$

Na Figura 2.13 (seção 2.6) temos $H_{\tau}$ simulada para $\phi=+0,80, \theta=-0,30$ e $\sigma_{a}^{2}=1$. Similar à f.a.c. do $\operatorname{ARMA}(1,1)$, esta função, comandada pelo seu ponto de sela, apresenta um decaimento exponencial para zero a partir do lag 1 .

\subsubsection{Estimador}

No caso de séries temporais, considerando um processo estocástico estacionário estrito e de segunda ordem, vimos em (2.1) que a f.a.c.l. $H_{\tau}\left(x_{1}, x_{2}\right)$ é dada pela expressão

$$
\begin{aligned}
H_{\tau}\left(x_{1}, x_{2}\right)= & \frac{\rho_{\tau}+\left(\left(\mu-E\left[X_{t} / X_{t+\tau}=x_{2}\right]\right) / \sigma\right)\left(\left(\mu-E\left[X_{t+\tau} / X_{t}=x_{1}\right]\right) / \sigma\right)}{\sqrt{1+\left(\left(\mu-E\left[X_{t} / X_{t+\tau}=x_{2}\right]\right) / \sigma\right)^{2}} \sqrt{1+\left(\left(\mu-E\left[X_{t+\tau} / X_{t}=x_{1}\right]\right) / \sigma\right)^{2}}}, \\
& \forall\left(x_{1}, x_{2}\right) \in S, \forall t, \tau \in Z, \tau \neq 0 .
\end{aligned}
$$

Então, os estimadores naturais para as componentes da medida passariam a ser, observados $\left(X_{1}, \ldots, X_{T}\right)$ :

$$
\begin{aligned}
& \bar{X}=\frac{1}{T} \sum_{t=1}^{T} X_{t} \text { para a média } \mu, \\
& c_{\tau}=\frac{1}{T} \sum_{t=1}^{T-\tau}\left(X_{t}-\bar{X}\right)\left(X_{t+\tau}-\bar{X}\right), \tau=0,1, \ldots, T-1 \text {, para a f.a.c.v. } \gamma_{\tau}, \text { em que } c_{-\tau}=c_{\tau},
\end{aligned}
$$




$$
\begin{aligned}
& r_{\tau}=\frac{c_{\tau}}{c_{0}}, \tau=0,1, \ldots, T-1 \text {, para a f.a.c. } \rho_{\tau}, \text { em que } r_{-\tau}=r_{\tau}, \\
& \hat{m}\left(x_{2} ; \tau\right)=\frac{\sum_{t=1}^{T-\tau} X_{t} K\left(\frac{x_{2}-X_{t+\tau}}{h_{T}}\right)}{\sum_{t=1}^{T-\tau} K\left(\frac{x_{2}-X_{t+\tau}}{h_{T}}\right)} \text { para } m\left(x_{2} ; \tau\right)=E\left[X_{t} / X_{t+\tau}=x_{2}\right],
\end{aligned}
$$

e

$$
\hat{m}\left(x_{1} ; \tau\right)=\frac{\sum_{t=1}^{T-\tau} X_{t+\tau} K\left(\frac{x_{1}-X_{t}}{h_{T}}\right)}{\sum_{t=1}^{T-\tau} K\left(\frac{x_{1}-X_{t}}{h_{T}}\right)} \text { para } m\left(x_{1} ; \tau\right)=E\left[X_{t+\tau} / X_{t}=x_{1}\right]
$$

sendo $K$ uma função kernel com caudas curtas e $h_{T}$ uma seqüência de larguras de faixa tendendo a zero numa taxa apropriada, com $\left(x_{1}, x_{2}\right) \in G$, em que $G$ é o conjunto dos pontos de grade. Observamos que os três primeiros estimadores são aqueles usualmente utilizados em séries temporais e os dois últimos são aqueles propostos independentemente por Nadaraya (1964) e Watson (1964) e cuja versão para séries temporais pode ser encontrada em Härdle et al. (1997, página 55).

\subsubsection{Consistência do estimador}

Fuller (1976) derivou a convergência em probabilidade do estimador da função de autocovariância amostral e, dentre outros, Bosq (1998) e Györfi et al. (1989) derivaram a convergência quase certa uniforme e a convergência completa uniforme ${ }^{(1)}$ (que implica na convergência quase certa), respectivamente, do estimador de Nadaraya (1964) e Watson (1964) para a esperança condicional. Antes de apresentar o teorema da consistência do estimador da f.a.c.l. $H_{\tau}\left(x_{1}, x_{2}\right)$, considere as seguintes condições de regularidade:

(C1) $X_{t}=\mu+\sum_{j=-\infty}^{+\infty} \alpha_{j} e_{t-j}$, em que $e_{t} \sim \operatorname{iid}\left(0, \sigma^{2}\right), E\left[e_{t}^{4}\right]=\eta \sigma^{4}, \sum_{j=-\infty}^{+\infty}\left|\alpha_{j}\right|<\infty$ e $\sum_{k=-\infty}^{\infty}\left|\gamma_{k}\right|<\infty$;

(C2) o parâmetro de suavização $h_{T}>0$, é tal que $h_{T} \rightarrow 0$ e $T h_{T} \rightarrow \infty$, quando $T \rightarrow \infty$;

(C3) o kernel $K$ é uma função densidade limitada, simétrica (em torno do zero) e tal que

$$
\lim _{x \rightarrow \infty} x K(x)=0 \text { e } \int x^{2} K(x) d x<+\infty ;
$$

(C4) $K$ é função contínua Lipschitziana de ordem $\gamma$ em $\mathfrak{R}$, ou seja, $\left|K\left(x_{1}\right)-K\left(x_{2}\right)\right| \leq c\left|x_{1}-x_{2}\right|^{\gamma}$ com $x_{1}, x_{2} \in \Re$ e $\gamma>0$;

(C5) $X_{t}$ é um processo $\alpha$-mixing, com coeficiente apresentando decaimento geométrico, ou seja, $\left.\exists u \in\right] 0 ; \infty[$ e $\exists v \in\left[0 ; 1\left[\right.\right.$ tal que $\alpha(k) \leq u v^{k}, k \geq 1$;

(1) Uma seqüência $\left\{X_{n}\right\}$ de elementos aleatórios é dita convergir completamente para um elemento aleatório $X$ (possivelmente degenerado), se para todo $\varepsilon>0, \sum_{n \geq 1} P\left[d\left(X_{n}, X\right)>\varepsilon\right]$ converge, sendo $d$ uma distância bem definida. 
(C6) $r$ e $f$ são funções duas vezes continuamente deriváveis e com valores em $\mathfrak{R}$, e tais que

$$
\begin{array}{ll}
\|f\|_{\infty}=\inf \{a: P(f>a)=0\} \leq b & \text { e }\left\|f^{(2)}\right\|_{\infty} \leq b, \\
\|r\|_{\infty}=\inf \{a: P(r>a)=0\} \leq b & \text { e }\left\|r^{(2)}\right\|_{\infty} \leq b,
\end{array}
$$

para algum $b$, em que $r\left(x_{1}\right)=\int x_{2} f\left(x_{1}, x_{2}\right) d x_{2}$ é o numerador da esperança condicional;

(C7) $E\left[\exp \left\{a\left|X_{t}\right|^{s}\right\}\right]<+\infty$, para algum $a>0$ e algum $s>0, \forall t \in Z$;

(C8) $\frac{T h_{T}}{(\log T)^{2+1 / s}} \rightarrow+\infty, T \rightarrow \infty, s>0$;

(C9) $S$ é um conjunto compacto tal que $\inf _{x \in S} f(x)>0$;

(C10) $h_{T} \cong\left(\frac{(\log T)^{2-(1 / s)}}{T}\right)^{1 / 5}, s>0$.

Teorema 2.1. Seja $\left\{X_{t}, t \in Z\right\}$ um processo estacionário estrito e de segunda ordem com as condições (C1) a (C10) satisfeitas. Então, temos que

$$
\hat{H}_{\tau}\left(x_{1}, x_{2}\right) \underset{T \rightarrow \infty}{\stackrel{P}{\longrightarrow}} H_{\tau}\left(x_{1}, x_{2}\right), \text { para cada } x_{1}, x_{2} \in S \text { fixados, }|\tau| \geq 1 .
$$

prova:

Por (C1) e pelo Corolário 6.1.1.2 de Fuller (1976), temos que $\bar{X}$ é um estimador consistente para $\mu$, e então

$$
\bar{X} \underset{T \rightarrow \infty}{\stackrel{P}{\longrightarrow}} \mu \text {. }
$$

Também, por (C1) e pelo Teorema 6.2.2 de Fuller (1976), $c_{\tau}$ é consistente para $\gamma_{\tau}$. Portanto,

$$
c_{\tau} \underset{T \rightarrow \infty}{\stackrel{P}{\longrightarrow}} \gamma_{\tau} .
$$

Pelo Teorema 3.2 de Bosq (1998), sendo $\left\{X_{t}\right\}$ um processo estacionário estrito com as condições (C3) a (C10) satisfeitas, então $\hat{m}(x ; \tau) \stackrel{q . c .}{\longrightarrow} m(x ; \tau)$, ou seja

$$
\hat{m}(x ; \tau) \underset{T \rightarrow \infty}{\stackrel{P}{\longrightarrow}} m(x ; \tau),
$$

para $h_{T} \rightarrow 0$ e $T h_{T} \rightarrow \infty$, ambos quando $T \rightarrow \infty$, para cada $x \in S$ fixado.

Considere

$$
\begin{aligned}
& \hat{\boldsymbol{\theta}}=\left(\bar{X}, c_{\tau}, c_{o}, \hat{m}\left(x_{2} ; \tau\right), \hat{m}\left(x_{1} ; \tau\right)\right)^{\prime}=\left(\hat{\theta}_{1}, \hat{\theta}_{2}, \hat{\theta}_{3}, \hat{\theta}_{4}, \hat{\theta}_{5}\right)^{\prime}, \\
& \boldsymbol{\theta}=\left(\mu, \gamma_{\tau}, \gamma_{o}, m\left(x_{2} ; \tau\right), m\left(x_{1} ; \tau\right)\right)^{\prime}=\left(\theta_{1}, \theta_{2}, \theta_{3}, \theta_{4}, \theta_{5}\right)^{\prime} .
\end{aligned}
$$

Verificamos que $\hat{\theta}_{i} \stackrel{P}{\longrightarrow} \theta_{i}, i=1,2, \ldots, 5$, para $h_{T} \rightarrow 0$ e $T h_{T} \rightarrow \infty$, ambos quando $T \rightarrow \infty, \forall x \in S$. Então, pelo Lema 5.1.3 de Fuller (1976), $\hat{\boldsymbol{\theta}} \stackrel{P}{\longrightarrow} \boldsymbol{\theta}$. Seja $g(\mathbf{y}): \mathfrak{R}^{5} \rightarrow \mathfrak{R}$ uma função contínua definida por 


$$
g(\mathbf{y})=\frac{y_{2} / y_{3}+\left(\left(y_{1}-y_{4}\right) / \sqrt{y_{3}}\right)\left(\left(y_{1}-y_{5}\right) / \sqrt{y_{3}}\right)}{\sqrt{1+\left(\left(y_{1}-y_{4}\right) / \sqrt{y_{3}}\right)^{2}} \sqrt{1+\left(\left(y_{1}-y_{5}\right) / \sqrt{y_{3}}\right)^{2}}}, \operatorname{com} \mathbf{y}=\left(y_{1}, y_{2}, y_{3}, y_{4}, y_{5}\right)^{\prime}, y_{3}>0 .
$$

Pelo Teorema 5.1 .4 de Fuller (1976), como $\hat{\boldsymbol{\theta}} \stackrel{P}{\longrightarrow} \boldsymbol{\theta}$ então $g(\hat{\boldsymbol{\theta}}) \stackrel{P}{\longrightarrow} g(\boldsymbol{\theta})$, portanto, $\hat{H}_{\tau}\left(x_{1}, x_{2}\right) \stackrel{P}{\longrightarrow} H_{\tau}\left(x_{1}, x_{2}\right)$, para $h_{T} \rightarrow 0$ e $T h_{T} \rightarrow \infty$, ambos quando $T \rightarrow \infty$, para cada $x_{1}, x_{2} \in S$ fixados.

\subsubsection{Simulações}

Para conhecermos o comportamento da f.a.c.l., elaboramos simulações da medida teórica $H_{\tau}\left(x_{1}, x_{2}\right)$ e do seu estimador $\hat{H}_{\tau}\left(x_{1}, x_{2}\right)$ para os modelos $\operatorname{AR}(1), \operatorname{MA}(1)$ e $\operatorname{ARMA}(1,1)$. Então, para alguns pontos da diagonal principal e da diagonal secundária da grade bidimensional, calculamos o viés e o erro quadrático médio, além do $p$-valor do teste de normalidade de Jarque Bera. Também, repetimos as simulações para diferentes tamanhos de séries para verificarmos o comportamento das propriedades desse estimador.

Considerando $\tau=1,2, \ldots, 6$, simulamos a medida teórica $H_{\tau}\left(x_{1}, x_{2}\right)$ pré especificando valores para os parâmetros de cada modelo em questão, e simulamos o seu estimador $\hat{H}_{\tau}\left(x_{1}, x_{2}\right)$ através de 200 replicações de Monte Carlo com séries de tamanho 500 (inicialmente) segundo o respectivo modelo, que contou com inovações Gaussianas de média 0 e variância 1. Os extremos da grade foram especificados através dos quantis teóricos de modo a obtermos $90 \%$ dos dados centrais. Diferentes funções kernels (Tabela 2.1) foram utilizadas, mostrando resultados muito similares. Então, nas simulações a seguir utilizamos o kernel Gaussiano, com parâmetro de suavização segundo a condição (C.10) do Teorema 1. A simulação foi elaborada no software R.

Tabela 2.1 - Funções kernels.

\begin{tabular}{|c|c|c|c|}
\hline Kernel & $\boldsymbol{K}(\boldsymbol{u})$ & Kernel & $\boldsymbol{K}(\boldsymbol{u})$ \\
\hline Epanechnikov & $\frac{3}{4}\left(1-u^{2}\right) I(|u| \leq 1)$ & Gaussiano & $\frac{1}{\sqrt{2 \pi}} \exp \left(-\frac{1}{2} u^{2}\right)$ \\
\hline Quártico & $\frac{15}{16}\left(1-u^{2}\right)^{2} I(|u| \leq 1)$ & Cosseno & $\frac{\pi}{4} \cos \left(\frac{\pi}{2} u\right) I(|u| \leq 1)$ \\
\hline Tri-ponderado & $\frac{35}{132}\left(1-u^{2}\right)^{3} I(|u| \leq 1)$ & \multicolumn{2}{|c}{} \\
\cline { 1 - 3 } & \multicolumn{2}{|r}{} &
\end{tabular}

O modelo AR(1) foi simulado considerando-se $\phi_{0}=0$ e $\phi_{1}=0,8$, ou seja

$$
X_{t}=0,8 X_{t-1}+a_{t}, \quad a_{t} \sim N(0,1) \text { independentes. }
$$

Analisando os gráficos da medida de dependência teórica para os lags de 1 a 6 (Figura 2.1), e de suas respectivas curvas de nível (Figura 2.2), observamos maior dependência positiva nos pontos de grade próximos à diagonal principal $\left(x_{1}=x_{2}\right)$ e dependência negativa (lags 1 a 3 ) ou aproximadamente nula (lags 4 
a 6) nos extremos próximos à diagonal secundária, sendo que a intensidade da dependência torna-se menor (aproxima-se de zero) quanto maior o lag. Além disso, podemos observar a presença de um decaimento exponencial para zero a partir do ponto de sela, indicando um comportamento similar à f.a.c. de um AR(1). Os gráficos da Figura 2.3 e Figura 2.4 referem-se à medida estimada, a qual mostra-se muito similar à medida teórica. Observando a Tabela 2.2, vemos que o viés é negativo para os pontos de grade da diagonal principal, e para vários pontos de grade centrais da diagonal secundária, indicando uma leve subestimação nestas regiões. Já o erro quadrático médio (Tabela 2.3) é pequeno para os diversos pontos de grade considerados. A Tabela 2.4 apresenta o $p$-valor do teste de normalidade de Jarque Bera, na qual verificamos, ao nível de significância de 5\%, que há maior incidência de rejeição da normalidade nos pontos de grade da diagonal principal do que nos pontos da diagonal secundária, além de ocorrer uma rejeição de normalidade mais destacada no ponto central da grade. A Figura 2.5 e a Figura 2.6 apresentam para o lag 3, os histogramas do estimador da medida nos pontos de grade da diagonal principal e da diagonal secundária, respectivamente, os quais indicam uma distribuição aproximadamente normal.

Três outras simulações foram elaboradas com 200 repetições de séries de tamanho $T=50, T=100 \mathrm{e}$ $T=200$, cujos resultados apresentam-se na Figura B.1 do Apêndice B. Comparando os resultados das simulações (inclusive para $T=500$ ), observamos que os gráficos das curvas de nível no lag 1 para $T=50 \mathrm{e}$ $T=500$, são muito similares entre si. Além disso, vemos que o erro quadrático médio e o viés (em módulo) diminuem com o aumento de $T$, assim como, de modo geral, diminui a rejeição da normalidade.

A simulação do modelo MA(1) foi elaborada considerando-se $\theta_{0}=0$ e $\theta_{1}=-0,8$, ou seja

$$
X_{t}=+0,8 a_{t-1}+a_{t}, a_{t} \sim N(0,1) \text { independentes. }
$$

Novamente, 200 séries de tamanho $T=500$ foram avaliadas. Na Figura 2.7 e 2.8, as quais referem-se à medida teórica, vemos que no lag 1 há dependência positiva nos pontos de grade da diagonal principal e dependência positiva e negativa nos pontos da diagonal secundária, enquanto que nos demais lags não há dependência, assim como ocorre com a f.a.c. do MA(1). Comparando-se estas figuras com as Figuras 2.9 e 2.10, vemos que a superfície de dependência estimada no lag 1 é um pouco mais suave que a teórica. Analisando a Tabela 2.5, observamos que no lag 1 há subestimação nos pontos extremos da grade da diagonal principal, e uma razoável superestimação da medida nos pontos de grade mais extremos da diagonal secundária. Nos demais lags, o viés mostra-se praticamente nulo. Na Tabela 2.6 temos os valores do erro quadrático médio, que apresentam-se com pequena magnitude, exceto nos pontos extremos da grade da diagonal secundária. $\mathrm{O} p$-valor do teste de normalidade de Jarque Bera apresenta-se na Tabela 2.7, sendo que no lag 1 não há rejeição da normalidade para os pontos de grade da diagonal principal, nem para os da diagonal secundária (exceto no primeiro ponto de grade). Para os demais lags, não houve rejeição da normalidade para mais da metade (quase metade) dos pontos de grade da diagonal principal (diagonal secundária). Para o lag 1, as figuras 2.11 e 2.12 apresentam os histogramas do estimador da medida nos pontos de grade da diagonal principal e da diagonal secundária, respectivamente, os quais geralmente não indicam forte afastamento da distribuição normal. 
Para este modelo também elaboramos simulações com 200 séries de tamanhos 50, 100 e 200 (ver Figura B. 2 do Apêndice B). Para $T=50$ e $T=500$, vemos grande semelhança entre os gráficos das curvas de nível (no lag 1). Observamos também, que com o aumento do tamanho da série, o erro quadrático médio e o viés (em módulo) diminuem, assim como ocorre maior incidência de não rejeição de normalidade nos pontos mais afastados do centro da grade.

A seguir, simulamos o modelo $\operatorname{ARMA}(1,1) \operatorname{com} \phi_{1}=+0,8, \theta_{1}=+0,3$, isto é

$$
X_{t}=+0,8 X_{t-1}-0,3 a_{t-1}+a_{t}, a_{t} \sim N(0,1) \text { independentes, }
$$

sendo que 200 séries de tamanho 500 foram consideradas inicialmente para elaborar a medida estimada. Analisando os gráficos das figuras 2.13 a 2.16, vemos que o comportamento teórico e estimado da medida é similar ao do modelo AR(1), porém, apresentando superfícies um pouco mais suaves em todos os lags. Também, ao longo dos lags, observamos um comportamento similar à f.a.c. do ARMA(1,1). Observamos na Tabela 2.8 que, de modo geral, há uma leve subestimação da medida. Já o erro quadrático médio apresentado na Tabela 2.9 é próximo de zero. Por fim, na Tabela 2.10 observamos que na maior parte das vezes, não há rejeição da normalidade do estimador. Os histogramas do estimador da medida no lag 3 apresentam-se nas figuras 2.17 e 2.18, os quais indicam distribuição aproximadamente normal.

Comparando os resultados desta simulação com as outras três simulações de 200 séries de tamanho 50, 100 e 200 (Figura B.3 no Apêndice B), além de verificarmos novamente que os gráficos das curvas de nível são similares entre si considerando a amostra menor e a maior, observamos que com o aumento do tamanho da série, o erro quadrático médio e o viés (em módulo) diminuem, assim como diminui a rejeição da normalidade nos pontos mais extremos da grade e nos pontos centrais (já para as amostras de tamanho 100 ou mais).

Agora, comparando o comportamento da f.a.c.l. estimada através de 200 repetições de séries de tamanho 500 para os três modelos considerados, temos que:

- o viés para o modelo AR(1) é negativo para todos os lags da diagonal principal, e geralmente positivo nos demais casos, principalmente nos pontos mais extremos da grade. Já o modelo MA(1) apresenta um comportamento inverso ao do modelo AR(1). O modelo ARMA(1,1) apresenta, de modo geral, um viés negativo, exceto nos pontos mais extremos dos três primeiros lags da diagonal principal;

- o erro quadrático médio é similar nos três modelos, exceto no lag 1 do modelo MA(1) que apresenta o maior erro quadrático médio, a menos dos pontos de grade mais centrais;

- ao nível de significância de 5\%, o maior índice de rejeição (menos de 50\% das vezes) da normalidade nos pontos de grade avaliados, ocorre no modelo MA(1), sendo que o modelo AR(1) apresenta alta incidência de rejeição da normalidade no ponto central da grade. 

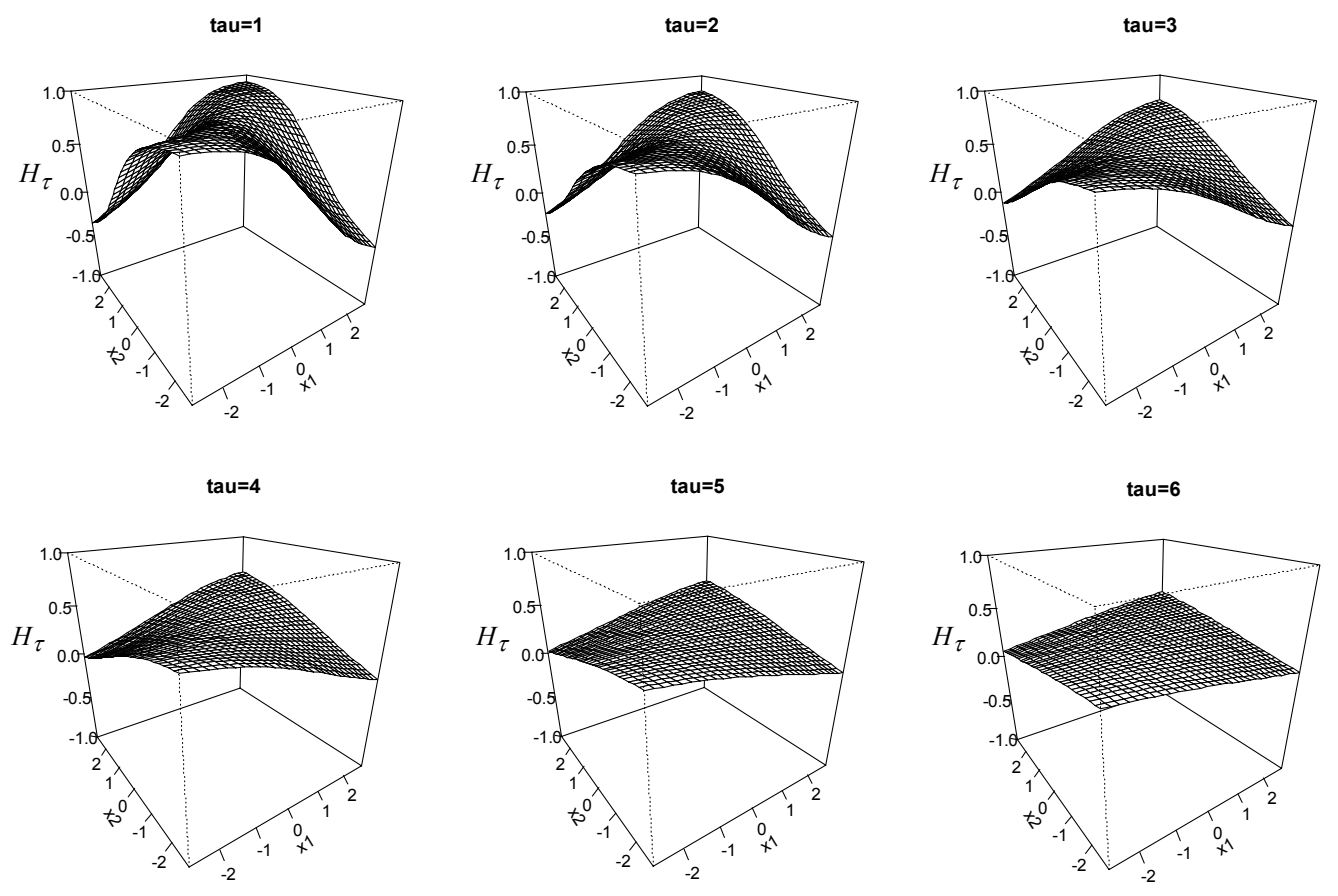

Figura 2.1 - Gráficos de perspectiva da f.a.c.l. teórica nos lags 1 a 6 para o modelo AR(1) simulado com 200 repetições de séries de tamanho $T=500$.
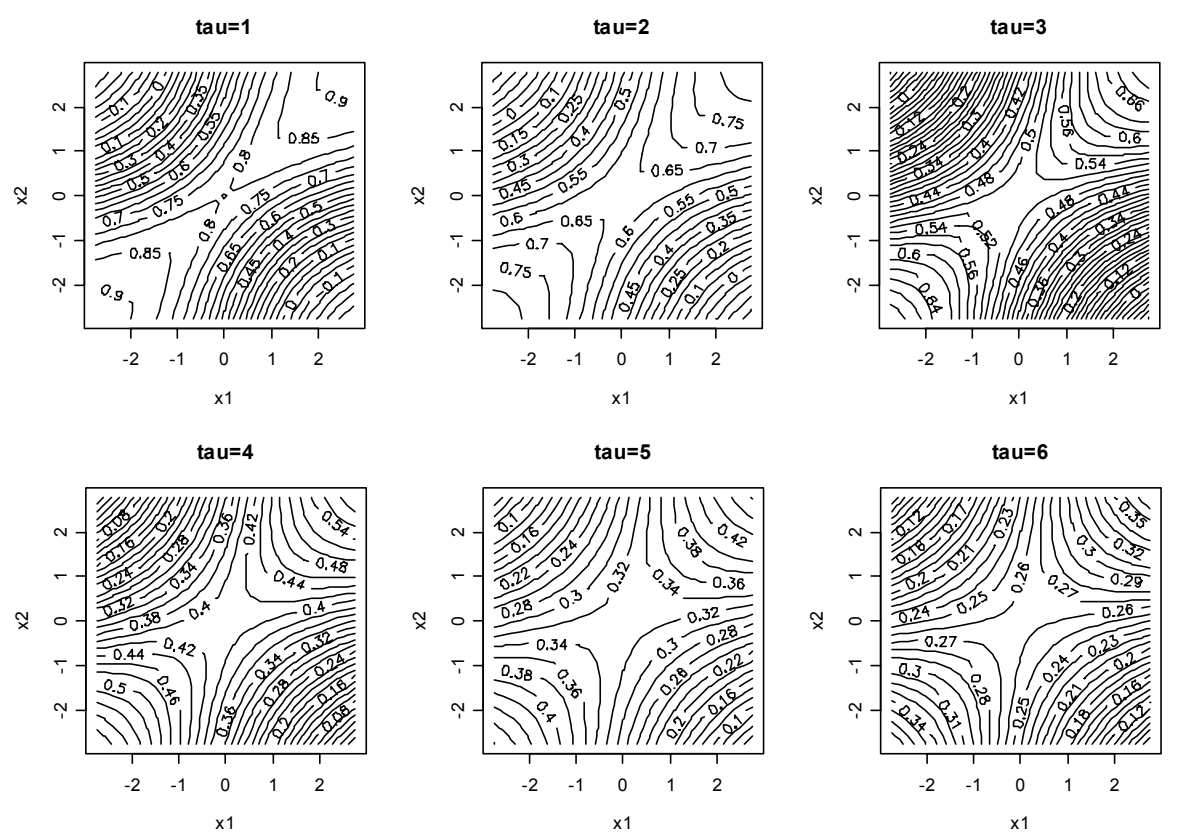

Figura 2.2 - Gráficos de curvas de nível da f.a.c.l. teórica nos lags 1 a 6 para o modelo AR(1) simulado com 200 repetições de séries de tamanho $T=500$. 

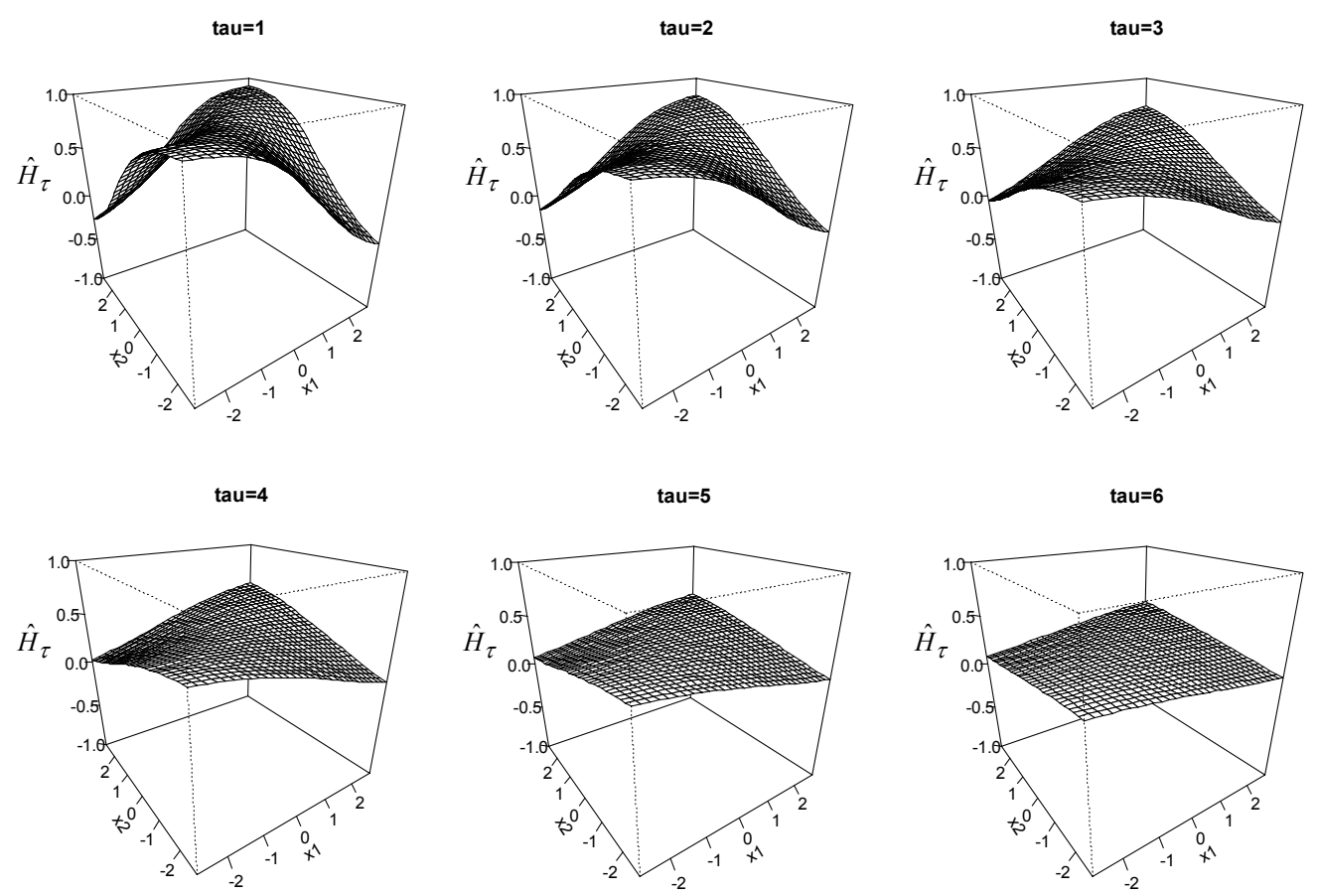

Figura 2.3 - Gráficos de perspectiva da f.a.c.l. estimada nos lags 1 a 6 para o modelo AR(1) simulado com 200 repetições de séries de tamanho $T=500$.
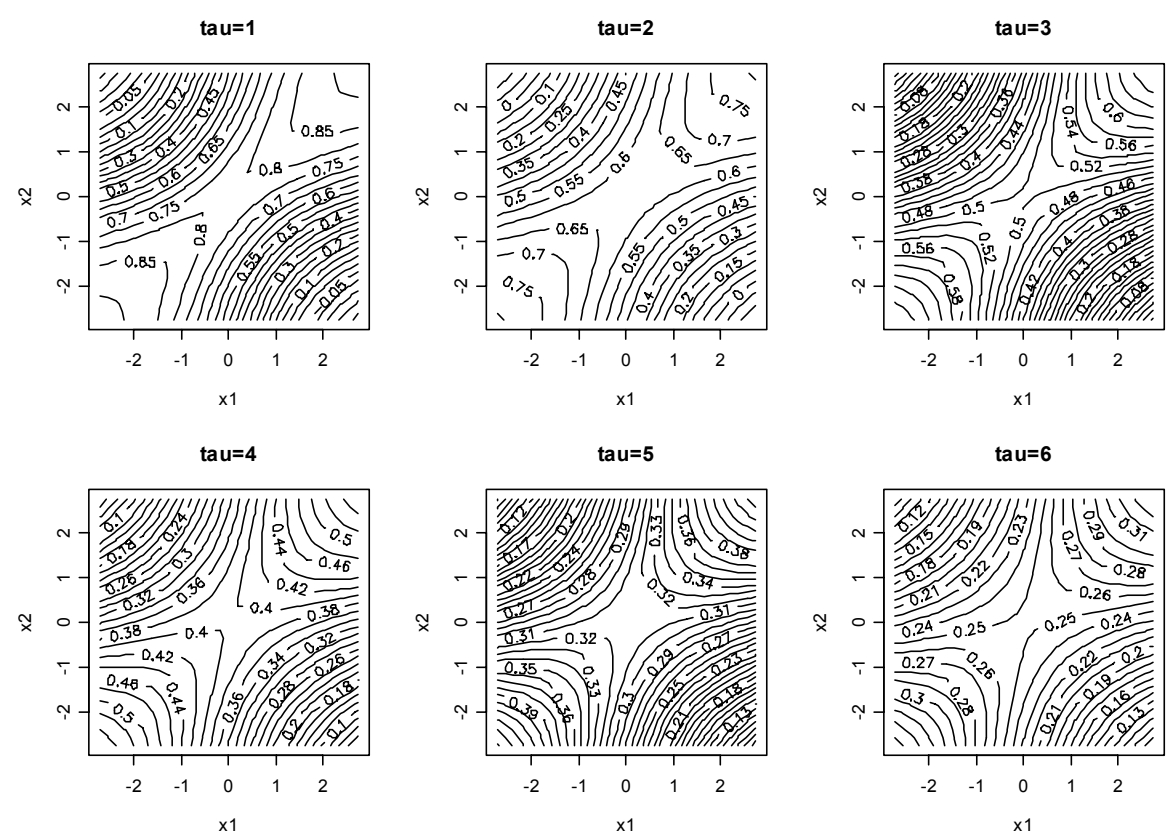

Figura 2.4 - Gráficos de curvas de nível da f.a.c.l. estimada nos lags 1 a 6 para o modelo AR(1) simulado com 200 repetições de séries de tamanho $T=500$. 
Tabela 2.2 - Viés da f.a.c.l. estimada para o modelo AR(1) simulado com 200 repetições de séries de tamanho $T=500$.

\begin{tabular}{|c|c|c|c|c|c|c|c|c|}
\hline Lag & Diagonal & $\mathbf{- 2 , 7 4}$ & $\mathbf{- 1 , 8 3}$ & $\mathbf{- 0 , 9 1}$ & $\mathbf{0 , 0 0}$ & $\mathbf{0 , 9 1}$ & $\mathbf{1 , 8 3}$ & $\mathbf{2 , 7 4}$ \\
\hline $\mathbf{1}$ & Principal & $-0,012$ & $-0,012$ & $-0,009$ & $-0,005$ & $-0,010$ & $-0,012$ & $-0,011$ \\
\hline $\mathbf{2}$ & Principal & $-0,025$ & $-0,021$ & $-0,014$ & $-0,009$ & $-0,015$ & $-0,022$ & $-0,024$ \\
\hline $\mathbf{3}$ & Principal & $-0,037$ & $-0,026$ & $-0,016$ & $-0,011$ & $-0,016$ & $-0,026$ & $-0,035$ \\
\hline $\mathbf{4}$ & Principal & $-0,040$ & $-0,026$ & $-0,015$ & $-0,011$ & $-0,015$ & $-0,026$ & $-0,041$ \\
\hline $\mathbf{5}$ & Principal & $-0,038$ & $-0,023$ & $-0,014$ & $-0,011$ & $-0,015$ & $-0,023$ & $-0,044$ \\
\hline $\mathbf{6}$ & Principal & $-0,033$ & $-0,020$ & $-0,014$ & $-0,011$ & $-0,014$ & $-0,022$ & $-0,038$ \\
\hline $\mathbf{1}$ & Secundária & 0,072 & 0,067 & 0,031 & $-0,005$ & 0,031 & 0,066 & 0,074 \\
\hline $\mathbf{2}$ & Secundária & 0,076 & 0,055 & 0,015 & $-0,009$ & 0,015 & 0,052 & 0,073 \\
\hline $\mathbf{3}$ & Secundária & 0,071 & 0,035 & 0,002 & $-0,011$ & 0,002 & 0,034 & 0,067 \\
\hline $\mathbf{4}$ & Secundária & 0,052 & 0,018 & $-0,004$ & $-0,011$ & $-0,005$ & 0,017 & 0,056 \\
\hline $\mathbf{5}$ & Secundária & 0,036 & 0,004 & $-0,008$ & $-0,011$ & $-0,009$ & 0,004 & 0,039 \\
\hline $\mathbf{6}$ & Secundária & 0,018 & $-0,006$ & $-0,010$ & $-0,011$ & $-0,010$ & $-0,004$ & 0,020 \\
\hline
\end{tabular}

Tabela 2.3 - Erro quadrático médio da f.a.c.l. estimada para o modelo AR(1) simulado com 200 repetições de séries de tamanho $T=500$.

\begin{tabular}{|c|c|c|c|c|c|c|c|c|}
\hline Lag & Diagonal & $\mathbf{- 2 , 7 4}$ & $\mathbf{- 1 , 8 3}$ & $\mathbf{- 0 , 9 1}$ & $\mathbf{0 , 0 0}$ & $\mathbf{0 , 9 1}$ & $\mathbf{1 , 8 3}$ & $\mathbf{2 , 7 4}$ \\
\hline $\mathbf{1}$ & Principal & 0,000 & 0,001 & 0,001 & 0,001 & 0,001 & 0,001 & 0,000 \\
\hline $\mathbf{2}$ & Principal & 0,002 & 0,002 & 0,002 & 0,002 & 0,002 & 0,002 & 0,002 \\
\hline $\mathbf{3}$ & Principal & 0,006 & 0,005 & 0,004 & 0,003 & 0,004 & 0,005 & 0,005 \\
\hline $\mathbf{4}$ & Principal & 0,011 & 0,008 & 0,006 & 0,005 & 0,006 & 0,007 & 0,010 \\
\hline $\mathbf{5}$ & Principal & 0,015 & 0,010 & 0,007 & 0,006 & 0,007 & 0,010 & 0,015 \\
\hline $\mathbf{6}$ & Principal & 0,018 & 0,012 & 0,008 & 0,007 & 0,008 & 0,011 & 0,017 \\
\hline $\mathbf{1}$ & Secundária & 0,007 & 0,007 & 0,003 & 0,001 & 0,003 & 0,007 & 0,008 \\
\hline $\mathbf{2}$ & Secundária & 0,009 & 0,006 & 0,002 & 0,002 & 0,003 & 0,005 & 0,009 \\
\hline $\mathbf{3}$ & Secundária & 0,009 & 0,004 & 0,002 & 0,003 & 0,003 & 0,004 & 0,009 \\
\hline $\mathbf{4}$ & Secundária & 0,008 & 0,003 & 0,003 & 0,005 & 0,003 & 0,003 & 0,007 \\
\hline $\mathbf{5}$ & Secundária & 0,005 & 0,003 & 0,004 & 0,006 & 0,004 & 0,002 & 0,005 \\
\hline $\mathbf{6}$ & Secundária & 0,003 & 0,003 & 0,005 & 0,007 & 0,005 & 0,003 & 0,003 \\
\hline
\end{tabular}

Tabela 2.4 - $P$-valor do teste de normalidade de Jarque Bera para a f.a.c.l. estimada para o modelo AR(1) simulado com 200 repetições de séries de tamanho $T=500$.

\begin{tabular}{|c|c|c|c|c|c|c|c|c|}
\hline Lag & Diagonal & $\mathbf{- 2 , 7 4}$ & $\mathbf{- 1 , 8 3}$ & $\mathbf{- 0 , 9 1}$ & $\mathbf{0 , 0 0}$ & $\mathbf{0 , 9 1}$ & $\mathbf{1 , 8 3}$ & $\mathbf{2 , 7 4}$ \\
\hline $\mathbf{1}$ & Principal & 0,026 & 0,059 & 0,099 & 0,008 & 0,364 & 0,317 & 0,508 \\
\hline $\mathbf{2}$ & Principal & 0,010 & 0,016 & 0,025 & 0,000 & 0,106 & 0,056 & 0,001 \\
\hline $\mathbf{3}$ & Principal & 0,001 & 0,013 & 0,045 & 0,008 & 0,063 & 0,009 & 0,001 \\
\hline $\mathbf{4}$ & Principal & 0,007 & 0,023 & 0,178 & 0,060 & 0,043 & 0,159 & 0,005 \\
\hline $\mathbf{5}$ & Principal & 0,057 & 0,270 & 0,353 & 0,254 & 0,350 & 0,589 & 0,293 \\
\hline $\mathbf{6}$ & Principal & 0,284 & 0,401 & 0,436 & 0,330 & 0,477 & 0,815 & 0,686 \\
\hline $\mathbf{1}$ & Secundária & 0,597 & 0,259 & 0,809 & 0,008 & 0,230 & 0,442 & 0,837 \\
\hline $\mathbf{2}$ & Secundária & 0,326 & 0,001 & 0,779 & 0,000 & 0,543 & 0,204 & 0,948 \\
\hline $\mathbf{3}$ & Secundária & 0,413 & 0,299 & 0,403 & 0,008 & 0,450 & 0,948 & 0,716 \\
\hline $\mathbf{4}$ & Secundária & 0,228 & 0,169 & 0,207 & 0,060 & 0,220 & 0,875 & 0,514 \\
\hline $\mathbf{5}$ & Secundária & 0,910 & 0,584 & 0,162 & 0,254 & 0,225 & 0,942 & 0,050 \\
\hline $\mathbf{6}$ & Secundária & 0,765 & 0,293 & 0,199 & 0,330 & 0,321 & 0,569 & 0,486 \\
\hline
\end{tabular}



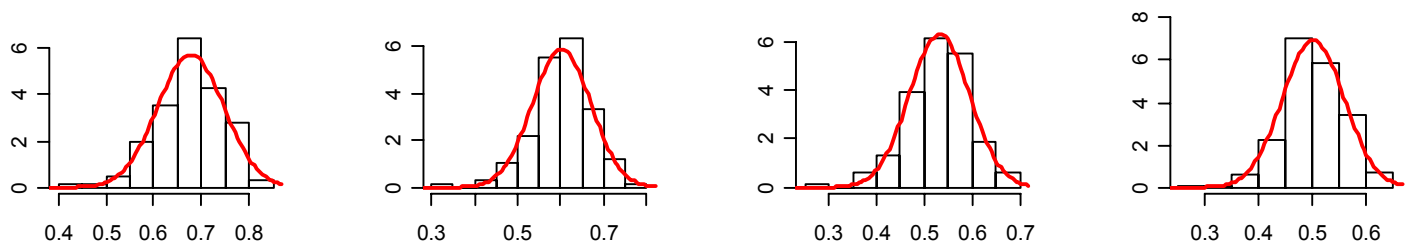

0.91

1.83

2.74
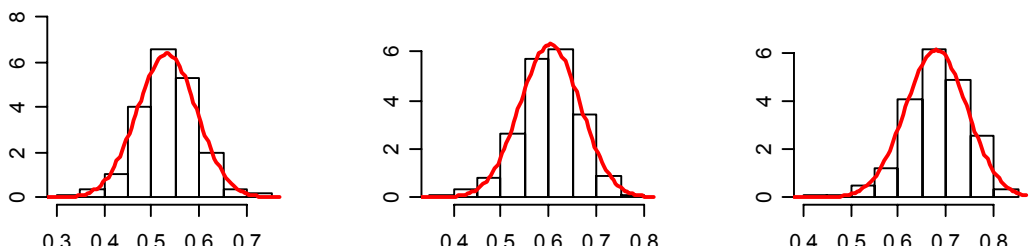

Figura 2.5 - Histogramas em pontos de grade da diagonal principal das f.a.c.l.'s estimadas no lag 3 para o modelo AR(1) simulado com 200 repetições de séries de tamanho $T=500$.

$-2.74$

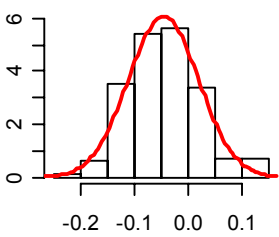

0.91

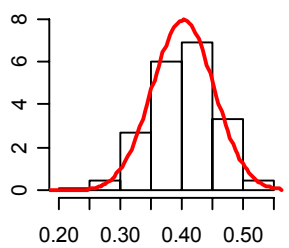

$-1.83$

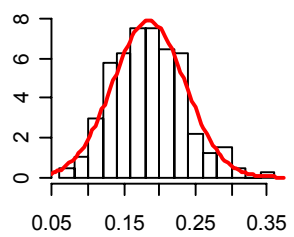

1.83

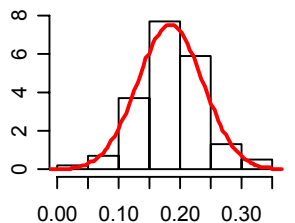

$-0.91$

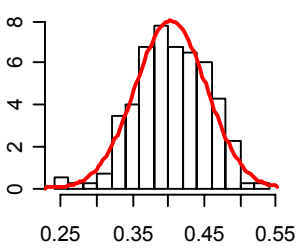

2.74

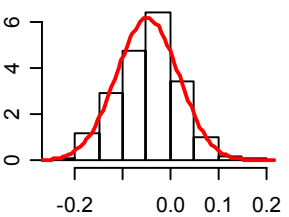

Figura 2.6 - Histogramas em pontos de grade da diagonal secundária das f.a.c.l.'s estimadas no lag 3 para o modelo AR(1) simulado com 200 repetições de séries de tamanho $T=500$. 

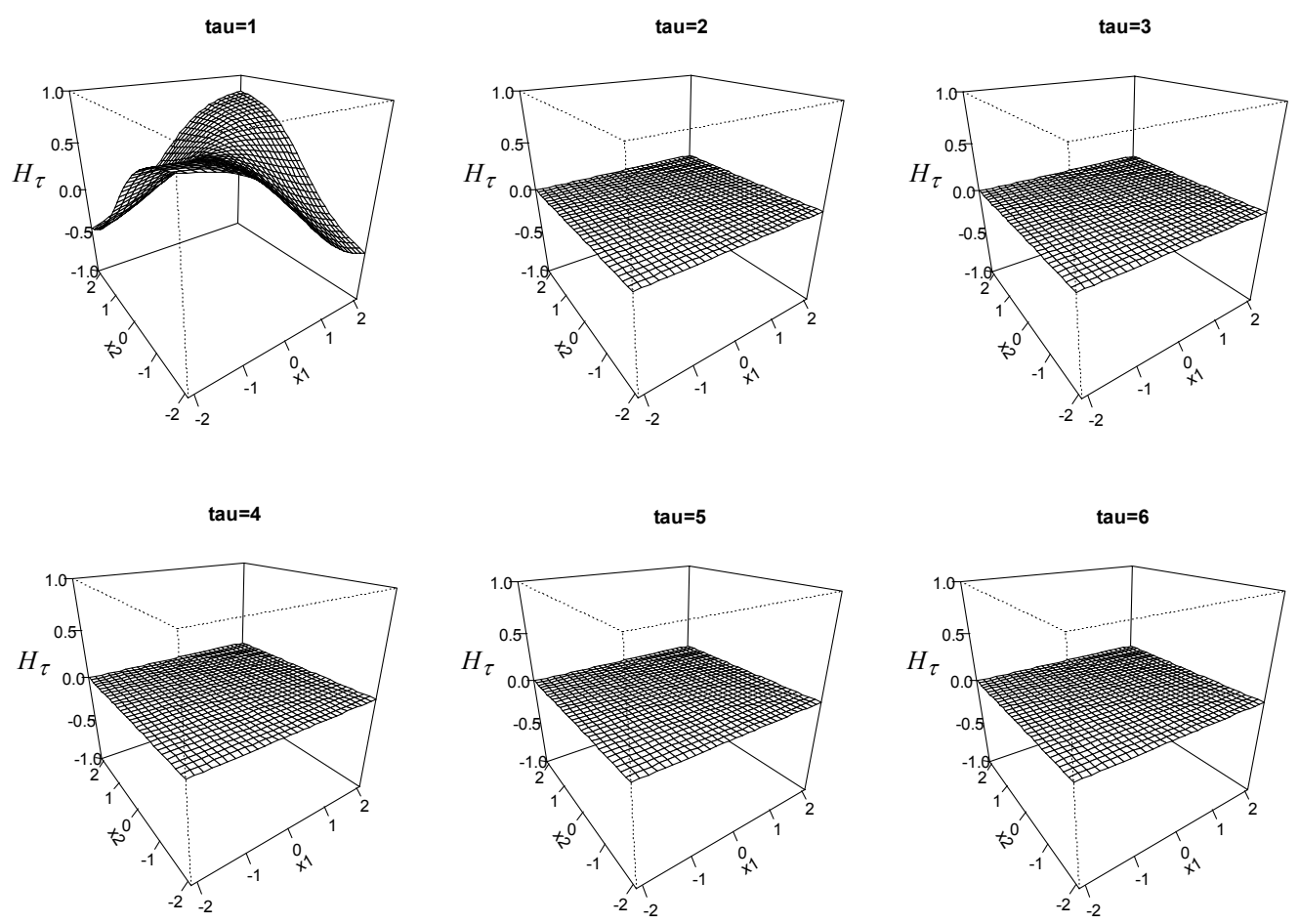

Figura 2.7 - Gráficos de perspectiva da f.a.c.l. teórica nos lags 1 a 6 para o modelo MA(1) simulado com 200 repetições de séries de tamanho $T=500$.
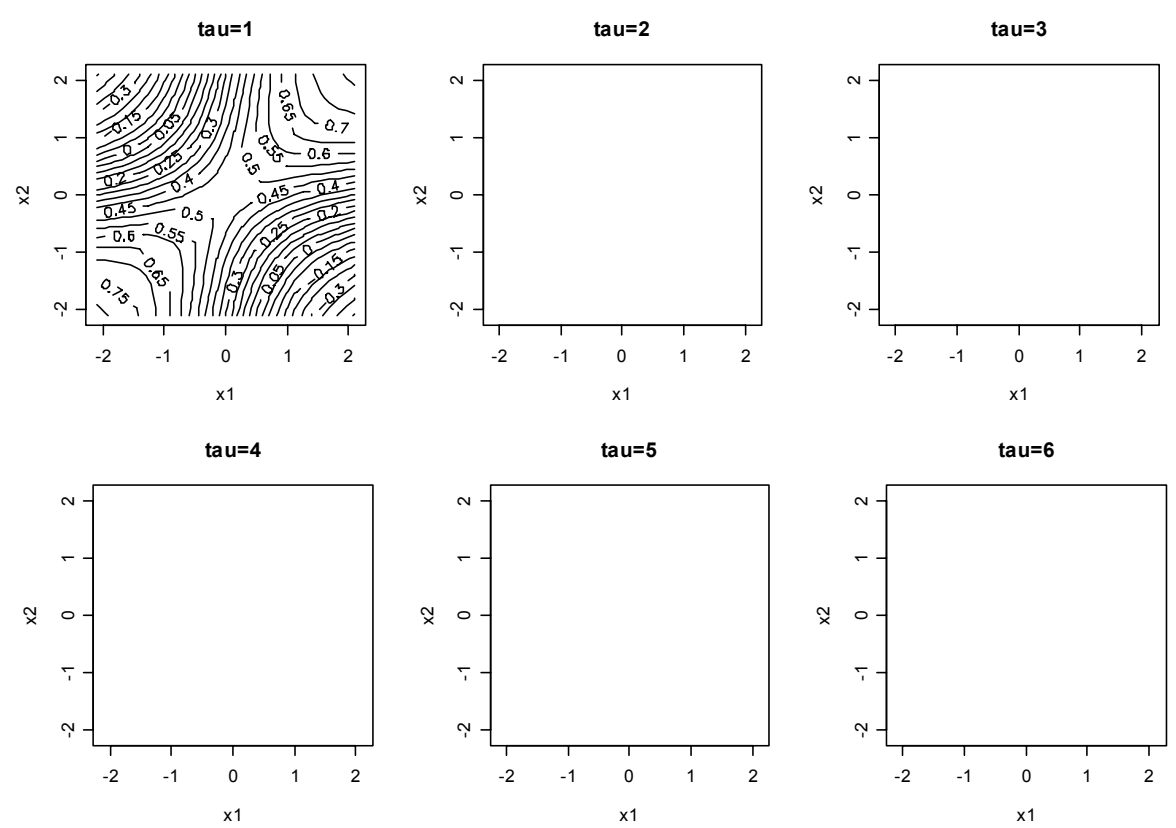

Figura 2.8 - Gráficos de curvas de nível da f.a.c.l. teórica nos lags 1 a 6 para o modelo MA(1) simulado com 200 repetições de séries de tamanho $T=500$. 

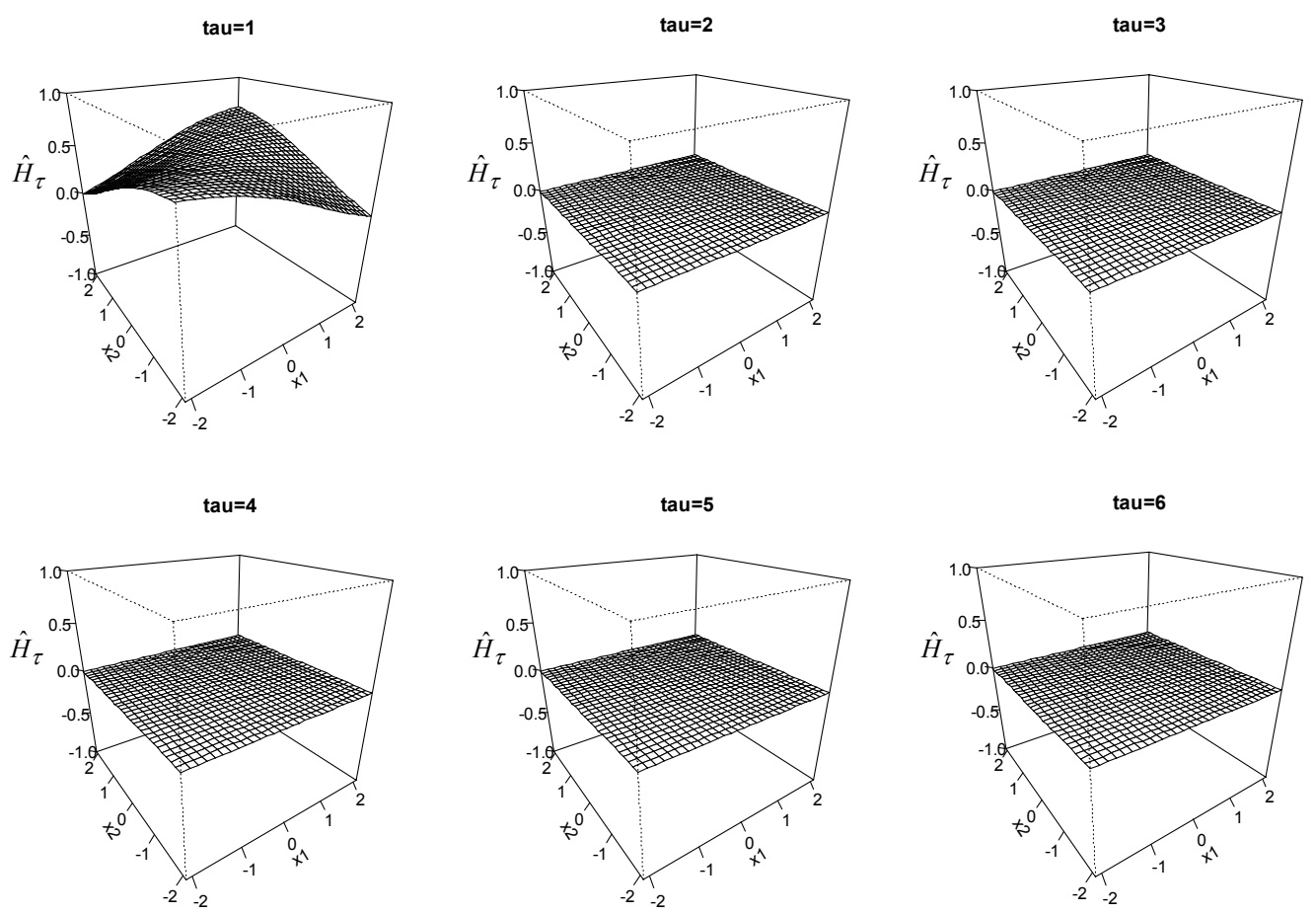

Figura 2.9 - Gráficos de perspectiva da fa.c.l. estimada nos lags 1 a 6 para o modelo MA(1) simulado com 200 repetições de séries de tamanho $T=500$.
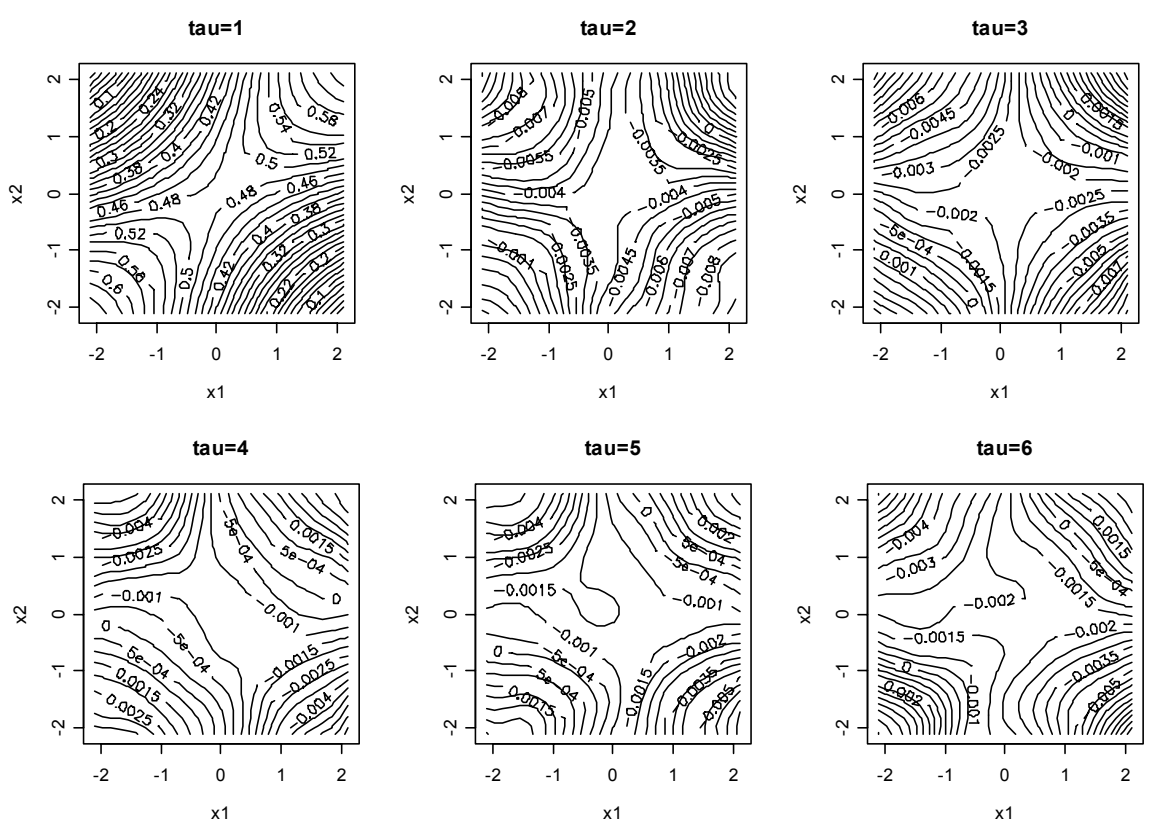

Figura 2.10 - Gráficos de curvas de nível da fa.c.l. estimada nos lags 1 a 6 para o modelo MA(1) simulado com 200 repetições de séries de tamanho $T=500$. 
Tabela 2.5 - Viés da f.a.c.l. estimada para o modelo MA(1) simulado com 200 repetições de séries de tamanho $T=500$.

\begin{tabular}{|c|c|c|c|c|c|c|c|c|}
\hline Lag & Diagonal & $\mathbf{- 2 , 1 1}$ & $\mathbf{- 1 , 4 0}$ & $\mathbf{- 0 , 7 0}$ & $\mathbf{0}$ & $\mathbf{0 , 7 0}$ & $\mathbf{1 , 4 0}$ & $\mathbf{2 , 1 1}$ \\
\hline $\mathbf{1}$ & Principal & $-0,156$ & $-0,132$ & $-0,060$ & $-0,004$ & $-0,060$ & $-0,134$ & $-0,160$ \\
\hline $\mathbf{2}$ & Principal & 0,002 & 0,000 & $-0,003$ & $-0,004$ & $-0,003$ & 0,000 & 0,004 \\
\hline $\mathbf{3}$ & Principal & 0,004 & 0,001 & $-0,002$ & $-0,002$ & $-0,002$ & 0,001 & 0,006 \\
\hline $\mathbf{4}$ & Principal & 0,004 & 0,002 & 0,000 & $-0,001$ & 0,000 & 0,002 & 0,004 \\
\hline $\mathbf{5}$ & Principal & 0,004 & 0,002 & $-0,001$ & $-0,002$ & $-0,001$ & 0,002 & 0,004 \\
\hline $\mathbf{6}$ & Principal & 0,006 & 0,002 & $-0,001$ & $-0,002$ & $-0,002$ & 0,001 & 0,003 \\
\hline $\mathbf{1}$ & Secundária & 0,440 & 0,366 & 0,156 & $-0,004$ & 0,157 & 0,370 & 0,443 \\
\hline $\mathbf{2}$ & Secundária & $-0,009$ & $-0,008$ & $-0,005$ & $-0,004$ & $-0,005$ & $-0,008$ & $-0,011$ \\
\hline $\mathbf{3}$ & Secundária & $-0,010$ & $-0,006$ & $-0,003$ & $-0,002$ & $-0,003$ & $-0,006$ & $-0,010$ \\
\hline $\mathbf{4}$ & Secundária & $-0,007$ & $-0,003$ & $-0,001$ & $-0,001$ & $-0,001$ & $-0,004$ & $-0,006$ \\
\hline $\mathbf{5}$ & Secundária & $-0,007$ & $-0,005$ & $-0,002$ & $-0,002$ & $-0,002$ & $-0,004$ & $-0,006$ \\
\hline $\mathbf{6}$ & Secundária & $-0,010$ & $-0,005$ & $-0,002$ & $-0,002$ & $-0,002$ & $-0,004$ & $-0,008$ \\
\hline
\end{tabular}

Tabela 2.6 - Erro quadrático médio da f.a.c.l. estimada para o modelo MA(1) simulado com 200 repetições de séries de tamanho $T=500$.

\begin{tabular}{|c|c|c|c|c|c|c|c|c|}
\hline Lag & Diagonal & $\mathbf{- 2 , 1 1}$ & $\mathbf{- 1 , 4 0}$ & $\mathbf{- 0 , 7 0}$ & $\mathbf{0}$ & $\mathbf{0 , 7 0}$ & $\mathbf{1 , 4 0}$ & $\mathbf{2 , 1 1}$ \\
\hline $\mathbf{1}$ & Principal & 0,026 & 0,019 & 0,005 & 0,001 & 0,005 & 0,019 & 0,027 \\
\hline $\mathbf{2}$ & Principal & 0,003 & 0,003 & 0,003 & 0,003 & 0,003 & 0,003 & 0,003 \\
\hline $\mathbf{3}$ & Principal & 0,004 & 0,003 & 0,003 & 0,003 & 0,003 & 0,003 & 0,004 \\
\hline $\mathbf{4}$ & Principal & 0,002 & 0,003 & 0,003 & 0,003 & 0,003 & 0,003 & 0,003 \\
\hline $\mathbf{5}$ & Principal & 0,003 & 0,003 & 0,003 & 0,003 & 0,003 & 0,003 & 0,003 \\
\hline $\mathbf{6}$ & Principal & 0,003 & 0,003 & 0,003 & 0,003 & 0,003 & 0,003 & 0,003 \\
\hline $\mathbf{1}$ & Secundária & 0,196 & 0,136 & 0,025 & 0,001 & 0,026 & 0,138 & 0,199 \\
\hline $\mathbf{2}$ & Secundária & 0,003 & 0,003 & 0,003 & 0,003 & 0,003 & 0,003 & 0,003 \\
\hline $\mathbf{3}$ & Secundária & 0,004 & 0,004 & 0,003 & 0,003 & 0,004 & 0,003 & 0,003 \\
\hline $\mathbf{4}$ & Secundária & 0,003 & 0,003 & 0,003 & 0,003 & 0,003 & 0,003 & 0,003 \\
\hline $\mathbf{5}$ & Secundária & 0,003 & 0,003 & 0,003 & 0,003 & 0,003 & 0,003 & 0,003 \\
\hline $\mathbf{6}$ & Secundária & 0,003 & 0,003 & 0,003 & 0,003 & 0,003 & 0,003 & 0,003 \\
\hline
\end{tabular}

Tabela 2.7 - $P$-valor do teste de normalidade de Jarque Bera para a f.a.c.l. estimada para o modelo MA(1) simulado com 200 repetições de séries de tamanho $T=500$.

\begin{tabular}{|c|c|c|c|c|c|c|c|c|}
\hline Lag & Diagonal & $\mathbf{- 2 , 1 1}$ & $\mathbf{- 1 , 4 0}$ & $\mathbf{- 0 , 7 0}$ & $\mathbf{0}$ & $\mathbf{0 , 7 0}$ & $\mathbf{1 , 4 0}$ & $\mathbf{2 , 1 1}$ \\
\hline $\mathbf{1}$ & Principal & 0,880 & 0,196 & 0,429 & 0,391 & 0,742 & 0,553 & 0,606 \\
\hline $\mathbf{2}$ & Principal & 0,000 & 0,002 & 0,082 & 0,324 & 0,124 & 0,000 & 0,000 \\
\hline $\mathbf{3}$ & Principal & 0,000 & 0,000 & 0,001 & 0,005 & 0,001 & 0,000 & 0,000 \\
\hline $\mathbf{4}$ & Principal & 0,275 & 0,756 & 0,137 & 0,058 & 0,078 & 0,733 & 0,110 \\
\hline $\mathbf{5}$ & Principal & 0,010 & 0,364 & 0,876 & 0,727 & 0,872 & 0,622 & 0,001 \\
\hline $\mathbf{6}$ & Principal & 0,001 & 0,647 & 0,854 & 0,611 & 0,871 & 0,736 & 0,119 \\
\hline $\mathbf{1}$ & Secundária & 0,005 & 0,668 & 0,348 & 0,391 & 0,185 & 0,656 & 0,120 \\
\hline $\mathbf{2}$ & Secundária & 0,006 & 0,369 & 0,561 & 0,324 & 0,529 & 0,219 & 0,012 \\
\hline $\mathbf{3}$ & Secundária & 0,000 & 0,178 & 0,012 & 0,005 & 0,016 & 0,153 & 0,064 \\
\hline $\mathbf{4}$ & Secundária & 0,000 & 0,000 & 0,023 & 0,058 & 0,012 & 0,000 & 0,000 \\
\hline $\mathbf{5}$ & Secundária & 0,002 & 0,089 & 0,610 & 0,727 & 0,749 & 0,039 & 0,000 \\
\hline $\mathbf{6}$ & Secundária & 0,000 & 0,015 & 0,429 & 0,611 & 0,488 & 0,015 & 0,000 \\
\hline
\end{tabular}


$-2.11$

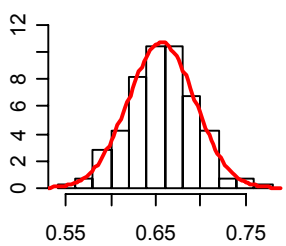

0.7

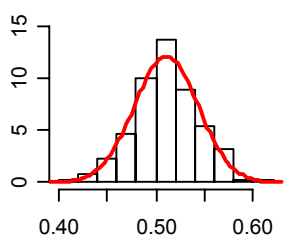

$-1.4$

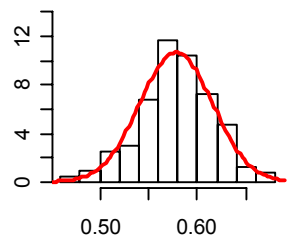

1.4

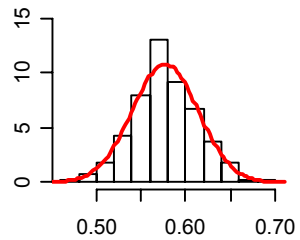

$-0.7$

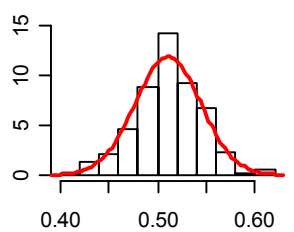

2.11

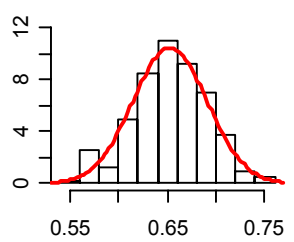

Figura 2.11 - Histogramas em pontos de grade da diagonal principal das f.a.c.l.'s estimadas no $\operatorname{lag} 1$ para o modelo MA(1) simulado com 200 repetições de séries de tamanho $T=500$.
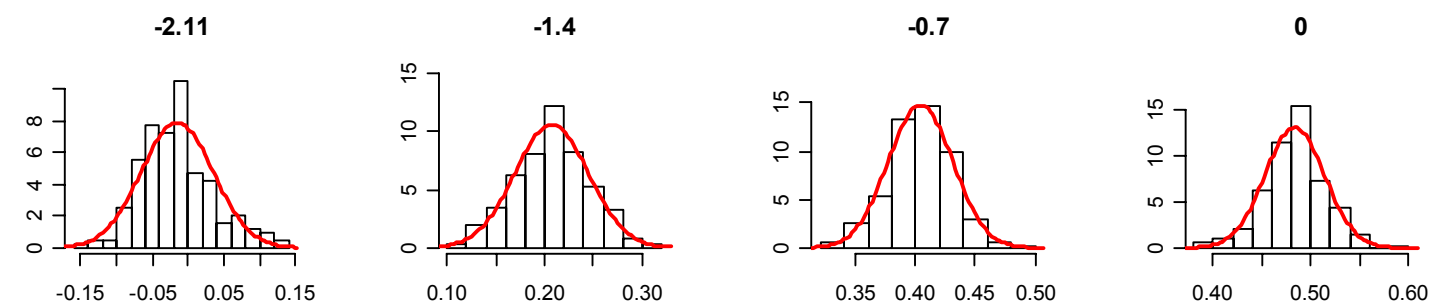

0.7

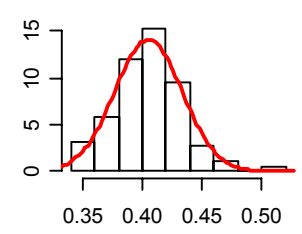

1.4

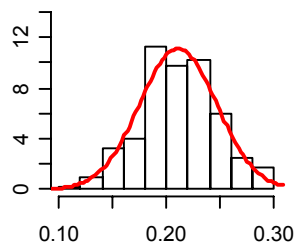

2.11

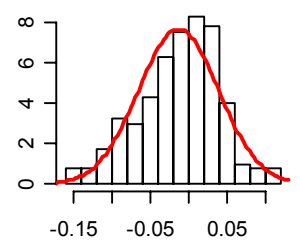

Figura 2.12 - Histogramas em pontos de grade da diagonal secundária das f.a.c.l.'s estimadas no lag 1 para o modelo MA(1) simulado com 200 repetições de séries de tamanho $T=500$. 

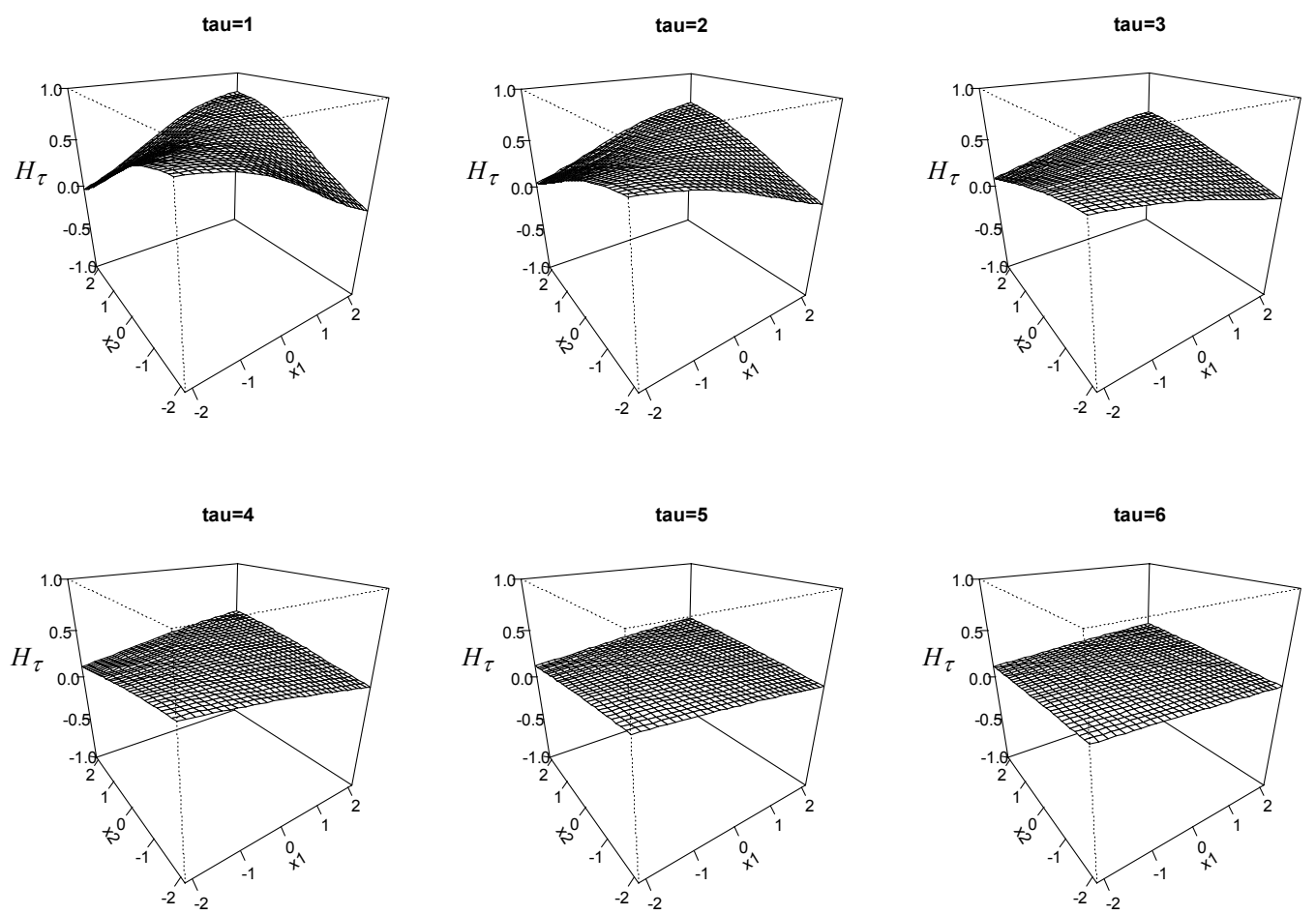

Figura 2.13 - Gráficos de perspectiva da f.a.c.l. teórica nos lags 1 a 6 para o modelo ARMA(1,1) simulado com 200 repetições de séries de tamanho $T=500$.
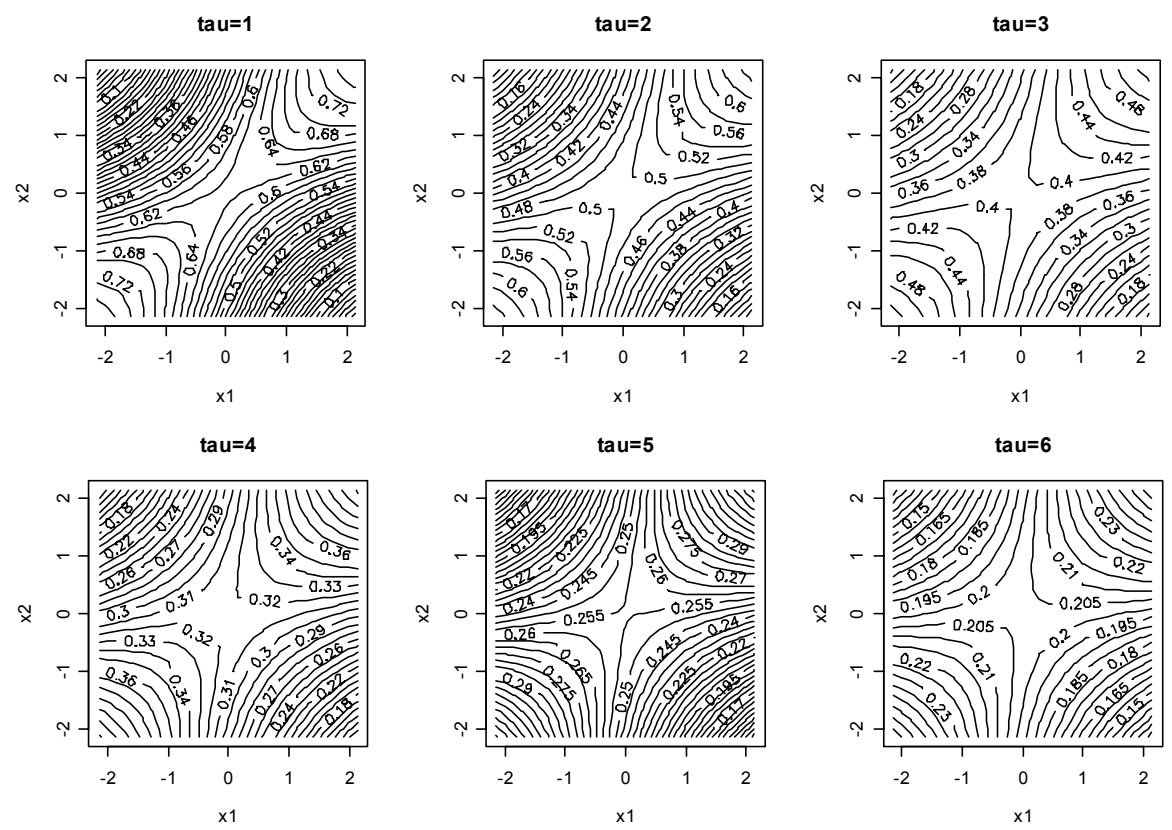

Figura 2.14 - Gráficos de curvas de nível da f.a.c.l. teórica nos lags 1 a 6 para o modelo ARMA(1,1) simulado com 200 repetições de séries de tamanho $T=500$. 

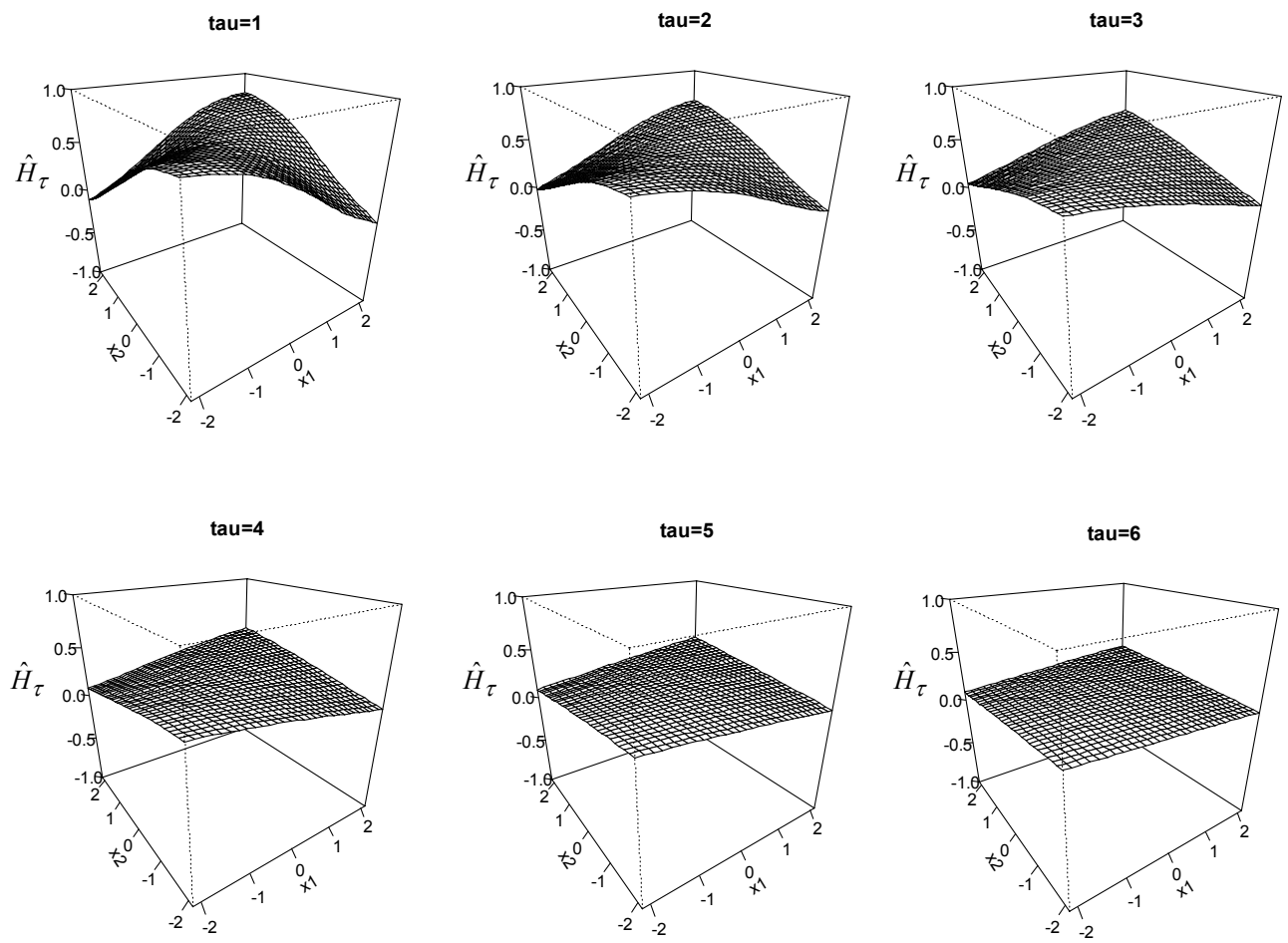

Figura 2.15 - Gráficos de perspectiva da f.a.c.l. estimada nos lags 1 a 6 para o modelo ARMA(1,1) simulado com 200 repetições de séries de tamanho $T=500$.
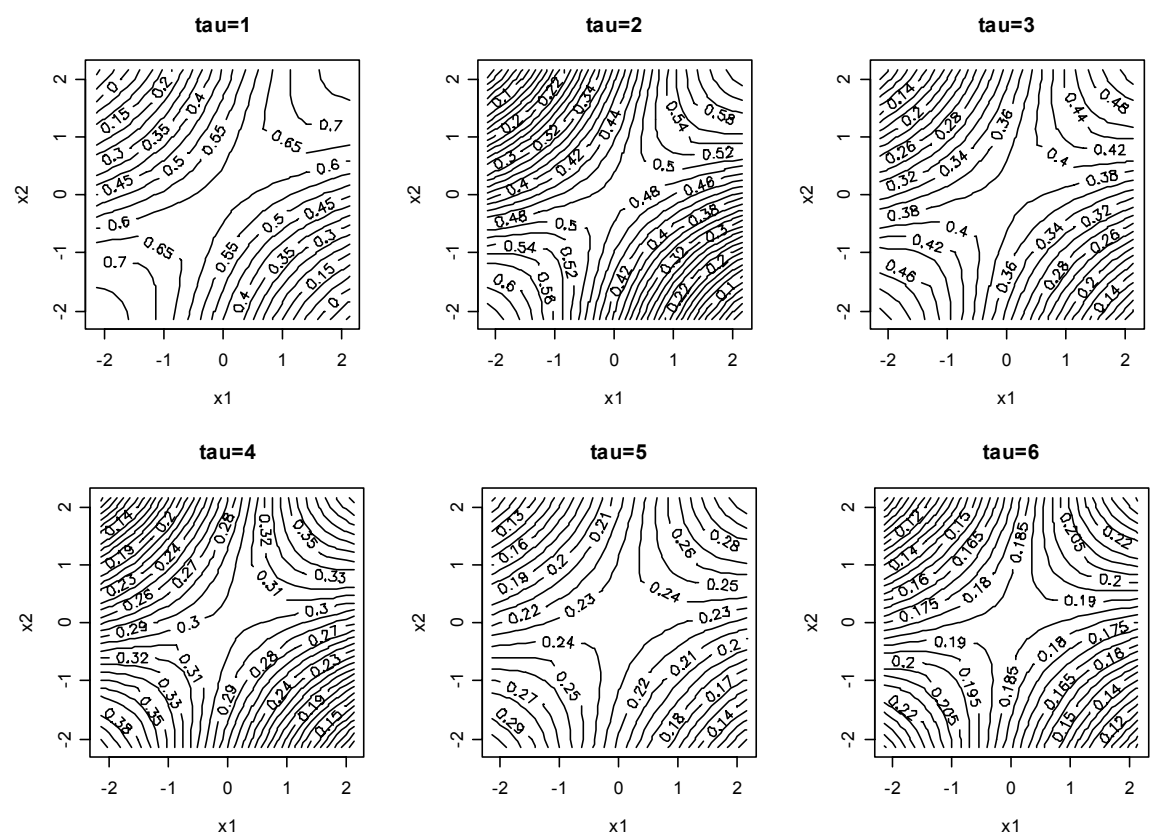

Figura 2.16 - Gráficos de curvas de nível da f.a.c.l. estimada nos lags 1 a 6 para o modelo ARMA(1,1) simulado com 200 repetições de séries de tamanho $T=500$. 
Tabela 2.8 - Viés da f.a.c.l. estimada para o modelo ARMA(1,1) simulado com 200 repetições de séries de tamanho $T=500$.

\begin{tabular}{|c|c|c|c|c|c|c|c|c|}
\hline Lag & Diagonal & $\mathbf{- 2 , 1 4}$ & $\mathbf{- 1 , 4 3}$ & $\mathbf{- 0 , 7 1}$ & $\mathbf{0 , 0 0}$ & $\mathbf{0 , 7 1}$ & $\mathbf{1 , 4 3}$ & $\mathbf{2 , 1 4}$ \\
\hline $\mathbf{1}$ & Principal & 0,011 & 0,006 & $-0,004$ & $-0,010$ & $-0,005$ & 0,003 & 0,008 \\
\hline $\mathbf{2}$ & Principal & 0,006 & 0,001 & $-0,008$ & $-0,013$ & $-0,010$ & $-0,001$ & 0,005 \\
\hline $\mathbf{3}$ & Principal & 0,001 & $-0,003$ & $-0,010$ & $-0,013$ & $-0,012$ & $-0,005$ & 0,003 \\
\hline $\mathbf{4}$ & Principal & $-0,007$ & $-0,010$ & $-0,015$ & $-0,017$ & $-0,016$ & $-0,013$ & $-0,008$ \\
\hline $\mathbf{5}$ & Principal & $-0,013$ & $-0,017$ & $-0,018$ & $-0,019$ & $-0,020$ & $-0,016$ & $-0,012$ \\
\hline $\mathbf{6}$ & Principal & $-0,012$ & $-0,015$ & $-0,016$ & $-0,017$ & $-0,017$ & $-0,014$ & $-0,012$ \\
\hline $\mathbf{1}$ & Secundária & $-0,079$ & $-0,067$ & $-0,033$ & $-0,010$ & $-0,033$ & $-0,066$ & $-0,080$ \\
\hline $\mathbf{2}$ & Secundária & $-0,065$ & $-0,057$ & $-0,026$ & $-0,013$ & $-0,027$ & $-0,048$ & $-0,064$ \\
\hline $\mathbf{3}$ & Secundária & $-0,052$ & $-0,039$ & $-0,020$ & $-0,013$ & $-0,021$ & $-0,039$ & $-0,052$ \\
\hline $\mathbf{4}$ & Secundária & $-0,038$ & $-0,033$ & $-0,021$ & $-0,017$ & $-0,021$ & $-0,033$ & $-0,043$ \\
\hline $\mathbf{5}$ & Secundária & $-0,034$ & $-0,028$ & $-0,021$ & $-0,019$ & $-0,022$ & $-0,031$ & $-0,040$ \\
\hline $\mathbf{6}$ & Secundária & $-0,029$ & $-0,026$ & $-0,020$ & $-0,017$ & $-0,020$ & $-0,027$ & $-0,034$ \\
\hline
\end{tabular}

Tabela 2.9 - Erro quadrático médio da f.a.c.l. estimada para o modelo ARMA $(1,1)$ simulado com 200 repetições de séries de tamanho $T=500$.

\begin{tabular}{|c|c|c|c|c|c|c|c|c|}
\hline Lag & Diagonal & $\mathbf{- 2 , 1 4}$ & $\mathbf{- 1 , 4 3}$ & $\mathbf{- 0 , 7 1}$ & $\mathbf{0 , 0 0}$ & $\mathbf{0 , 7 1}$ & $\mathbf{1 , 4 3}$ & $\mathbf{2 , 1 4}$ \\
\hline $\mathbf{1}$ & Principal & 0,002 & 0,002 & 0,002 & 0,002 & 0,002 & 0,002 & 0,002 \\
\hline $\mathbf{2}$ & Principal & 0,004 & 0,004 & 0,003 & 0,003 & 0,004 & 0,004 & 0,004 \\
\hline $\mathbf{3}$ & Principal & 0,007 & 0,006 & 0,005 & 0,004 & 0,005 & 0,006 & 0,008 \\
\hline $\mathbf{4}$ & Principal & 0,011 & 0,008 & 0,006 & 0,005 & 0,006 & 0,007 & 0,011 \\
\hline $\mathbf{5}$ & Principal & 0,013 & 0,008 & 0,006 & 0,006 & 0,006 & 0,008 & 0,012 \\
\hline $\mathbf{6}$ & Principal & 0,013 & 0,009 & 0,006 & 0,006 & 0,007 & 0,008 & 0,013 \\
\hline $\mathbf{1}$ & Secundária & 0,008 & 0,007 & 0,003 & 0,002 & 0,003 & 0,007 & 0,009 \\
\hline $\mathbf{2}$ & Secundária & 0,007 & 0,005 & 0,003 & 0,003 & 0,003 & 0,005 & 0,007 \\
\hline $\mathbf{3}$ & Secundária & 0,006 & 0,003 & 0,003 & 0,004 & 0,003 & 0,003 & 0,006 \\
\hline $\mathbf{4}$ & Secundária & 0,004 & 0,003 & 0,004 & 0,005 & 0,004 & 0,003 & 0,004 \\
\hline $\mathbf{5}$ & Secundária & 0,003 & 0,003 & 0,005 & 0,006 & 0,005 & 0,003 & 0,003 \\
\hline $\mathbf{6}$ & Secundária & 0,003 & 0,003 & 0,005 & 0,006 & 0,005 & 0,003 & 0,003 \\
\hline
\end{tabular}

Tabela 2.10 - $P$-valor do teste de normalidade de Jarque Bera para a f.a.c.l. estimada para o modelo ARMA(1,1) simulado com 200 repetições de séries de tamanho $T=500$.

\begin{tabular}{|c|c|c|c|c|c|c|c|c|}
\hline Lag & Diagonal & $\mathbf{- 2 , 1 4}$ & $\mathbf{- 1 , 4 3}$ & $\mathbf{- 0 , 7 1}$ & $\mathbf{0 , 0 0}$ & $\mathbf{0 , 7 1}$ & $\mathbf{1 , 4 3}$ & $\mathbf{2 , 1 4}$ \\
\hline $\mathbf{1}$ & Principal & 0,000 & 0,000 & 0,218 & 0,348 & 0,245 & 0,050 & 0,000 \\
\hline $\mathbf{2}$ & Principal & 0,000 & 0,015 & 0,232 & 0,188 & 0,302 & 0,111 & 0,017 \\
\hline $\mathbf{3}$ & Principal & 0,002 & 0,014 & 0,044 & 0,045 & 0,062 & 0,003 & 0,000 \\
\hline $\mathbf{4}$ & Principal & 0,659 & 0,590 & 0,602 & 0,613 & 0,867 & 0,673 & 0,607 \\
\hline $\mathbf{5}$ & Principal & 0,775 & 0,926 & 0,861 & 0,854 & 0,937 & 0,481 & 0,496 \\
\hline $\mathbf{6}$ & Principal & 0,370 & 0,316 & 0,281 & 0,222 & 0,278 & 0,379 & 0,599 \\
\hline $\mathbf{1}$ & Secundária & 0,292 & 0,145 & 0,494 & 0,348 & 0,612 & 0,764 & 0,644 \\
\hline $\mathbf{2}$ & Secundária & 0,168 & 0,476 & 0,460 & 0,188 & 0,504 & 0,021 & 0,949 \\
\hline $\mathbf{3}$ & Secundária & 0,383 & 0,198 & 0,085 & 0,045 & 0,131 & 0,077 & 0,055 \\
\hline $\mathbf{4}$ & Secundária & 0,606 & 0,592 & 0,464 & 0,613 & 0,605 & 0,507 & 0,221 \\
\hline $\mathbf{5}$ & Secundária & 0,814 & 0,554 & 0,686 & 0,854 & 0,826 & 0,250 & 0,475 \\
\hline $\mathbf{6}$ & Secundária & 0,005 & 0,189 & 0,172 & 0,222 & 0,267 & 0,027 & 0,110 \\
\hline
\end{tabular}


$-2.14$

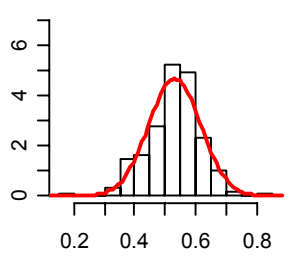

0.71

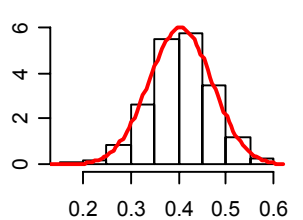

$-1.43$

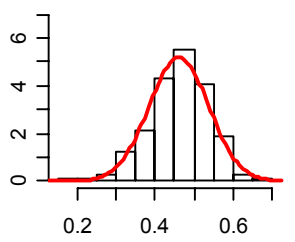

1.43

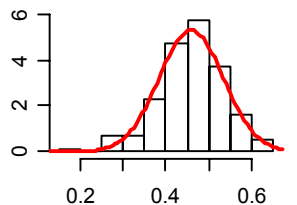

$-0.71$

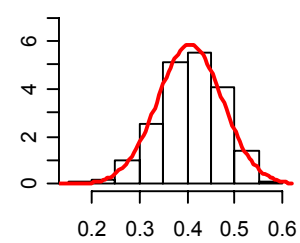

2.14

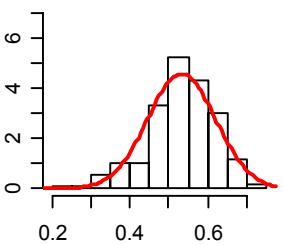

Figura 2.17 - Histogramas em pontos de grade da diagonal principal das f.a.c.l.'s estimadas no lag 3 para o modelo ARMA(1,1) simulado com 200 repetições de séries de tamanho $T=500$.

$-2.14$

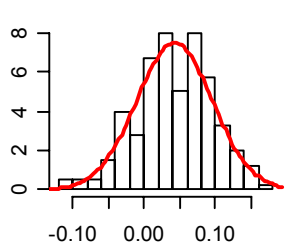

0.71

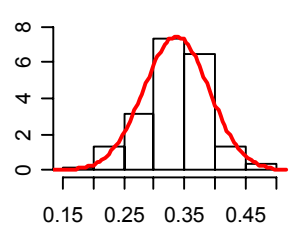

$-1.43$

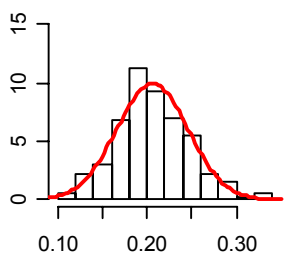

1.43

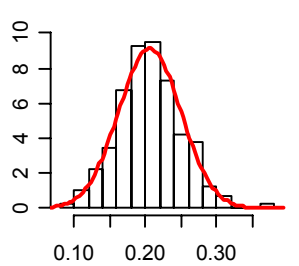

$-0.71$

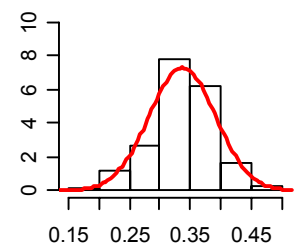

2.14

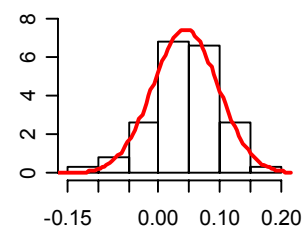

Figura 2.18 - Histogramas em pontos de grade da diagonal secundária das f.a.c.l.'s estimadas no lag 3 para o modelo ARMA $(1,1)$ simulado com 200 repetições de séries de tamanho $T=500$. 


\subsubsection{Aplicações a séries reais}

A seguir, apresenta-se a análise da estrutura de dependência local de duas séries financeiras (retornos) utilizando a função de autocorrelação local, e comparando-a com as ferramentas usuais (f.a.c. dos retornos e f.a.c. dos retornos ao quadrado), além de outra medida de dependência local, no caso, a densidade cópula empírica suavizada via kernel (em que o valores $1,>1$ e $<1$ indicam independência local, dependência positiva local e dependência negativa local, nesta ordem) e respectivos gráficos de dispersão dos postos normalizados (em que a distribuição aleatória desses pontos indica independência entre as duas variáveis aleatórias) e de curvas de nível. As séries avaliadas foram os retornos (ou log-retornos) diários da Petrobrás no período de 3/jan/95 a 27/dez/00, totalizando 1.498 dados, e também os retornos diários do Banespa no mesmo período.

Para estimar a f.a.c.l., utilizamos o kernel Gaussiano com largura de faixa igual ao desvio-padrão amostral dos dados, conforme sugestão de Bjerve e Doksum (1993), e 99,4\% dos dados centrais. Observamos que a largura de faixa sugerida por Ruppert et al. (1995) também foi avaliada em todas as simulações, mostrando resultados similares.

Primeiramente, vamos analisar os retornos diários da Petrobrás. Na Figura 2.19, podemos observar inicialmente o gráfico dos retornos ao longo do tempo e a distribuição dos retornos através do histograma. Em seguida, vemos a f.a.c. dos retornos a qual indica a existência de correlação serial, e a f.a.c. dos retornos ao quadrado que claramente sugere haver dependência entre os retornos. Na Figura 2.20, para os quatro primeiros lags, observamos inicialmente o gráfico de dispersão dos retornos, os quais corroboram a pequena relação de lag 1 existente nos dados. Nos quatro gráficos a seguir, vemos a f.a.c.l. dos retornos, sendo que no lag 1 há clara indicação de dependência positiva local (regiões da superfície acima do plano que corta o eixo vertical no zero). Nos três lags seguintes, parece haver leve dependência negativa ou positiva local. Nos últimos gráficos desta figura, encontram-se as respectivas curvas de nível. Na Figura 2.21, novamente para os lags de $1 \mathrm{a} 4$, apresentamos os gráficos de dispersão dos postos normalizados, sendo que no primeiro lag observamos dependência entre os valores baixos dos postos normalizados. Os quatro gráficos seguintes mostram a densidade cópula empírica suavizada, que mais destacadamente indica dependência positiva entre baixos valores no lag 1, mostrando comportamento similar ao da f.a.c.1..

Agora, analisando os retornos diários do Banespa, através da Figura 2.22 vemos que há correlação positiva no lag 1, e também há evidências de dependência entre os retornos indicada através da fa.c. dos retornos ao quadrado. Ao observarmos os gráficos das f.a.c.l.'s e de suas respectivas curvas de nível na Figura 2.23, podemos observar uma suave dependência positiva no lag 1 entre os retornos de valores maiores, principalmente. Este comportamento é confirmado através dos gráficos de dispersão dos postos normalizados, e também da densidade cópula empírica suavizada e suas curvas de nível correspondentes (Figura 2.24). 


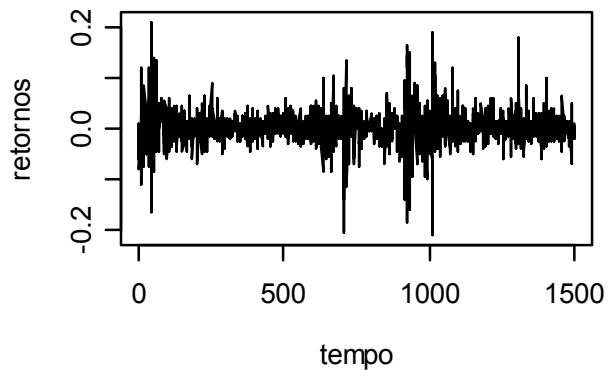

retornos

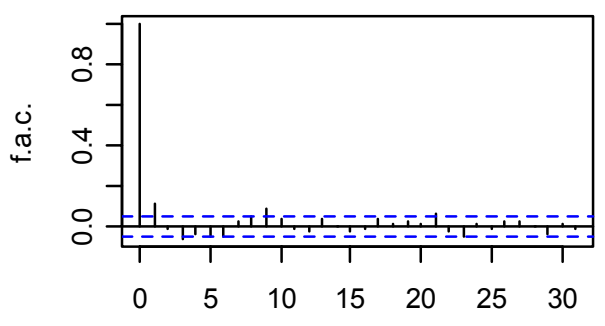

lag

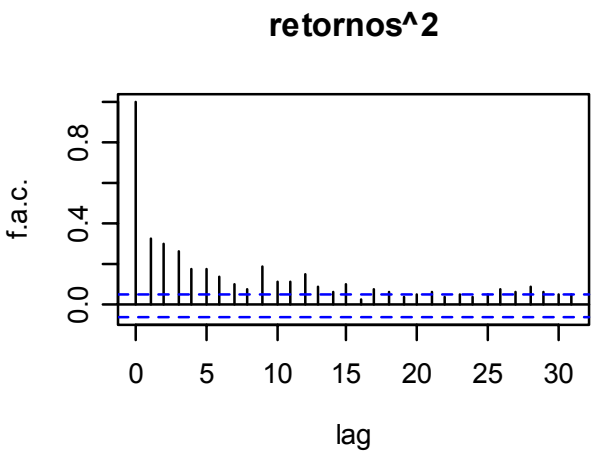

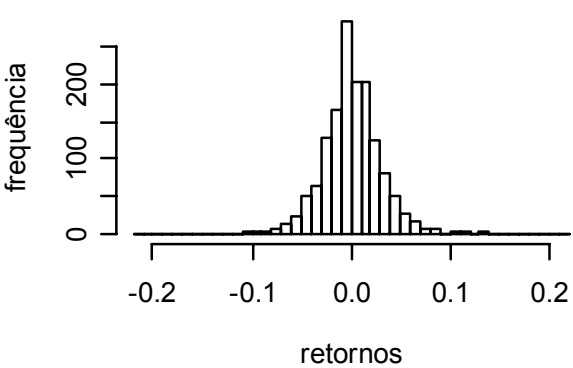

retornos

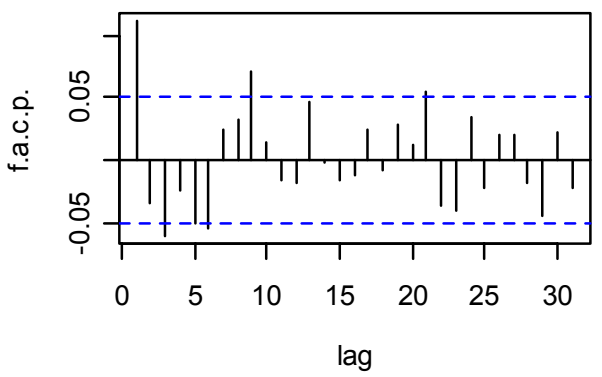

retornos^ 2

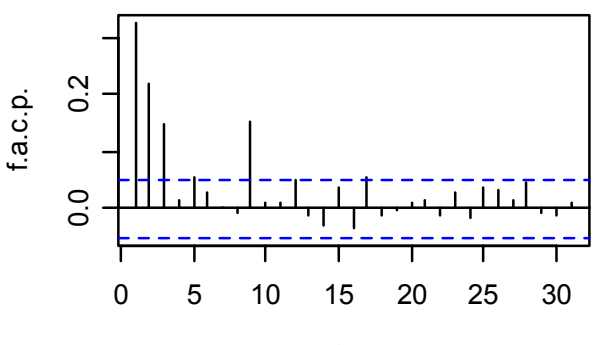

lag

Figura 2.19 - Gráfico no tempo, histograma, f.a.c. e f.a.c.p. dos retornos, além da f.a.c. e f.a.c.p. dos retornos ao quadrado, respectivamente. Os retornos referem-se aos retornos diários da Petrobrás de 3/jan/95 a 27/dez/00. 

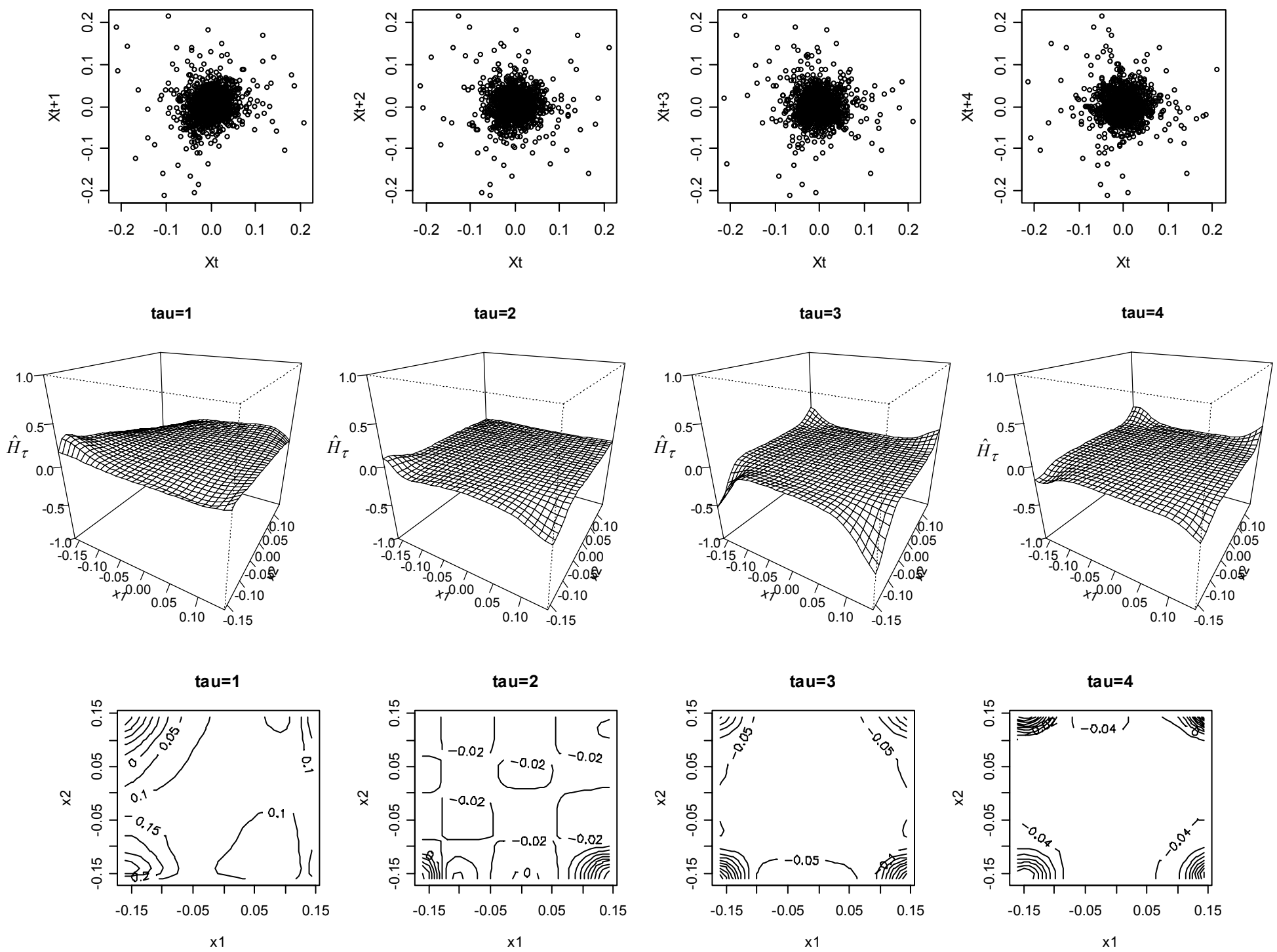

Figura 2.20 - Gráficos de dispersão, da f.a.c.l., e de curvas de nível da f.a.c.l. (considerando os lags de 1 a 4), respectivamente, dos retornos diários da Petrobrás de 3/jan/95 a 27/dez/00. 


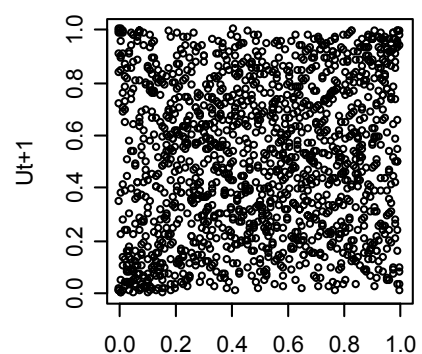

Ut

tau $=1$

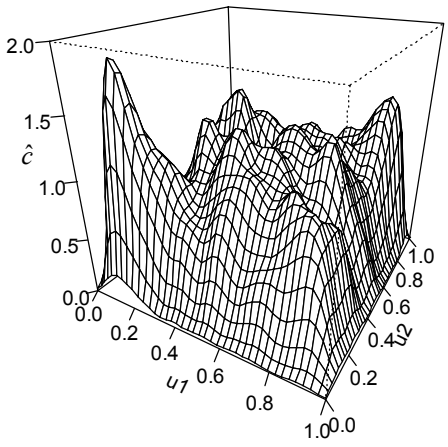

tau $=1$

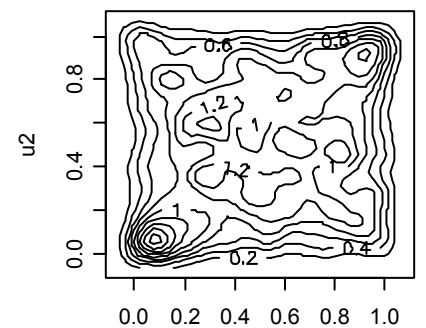

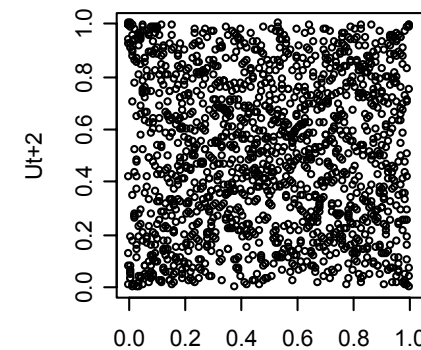

$\mathrm{U}$

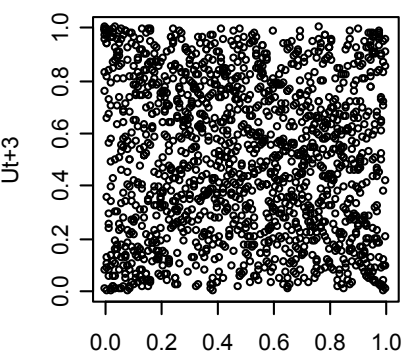

Ut

tau $=3$

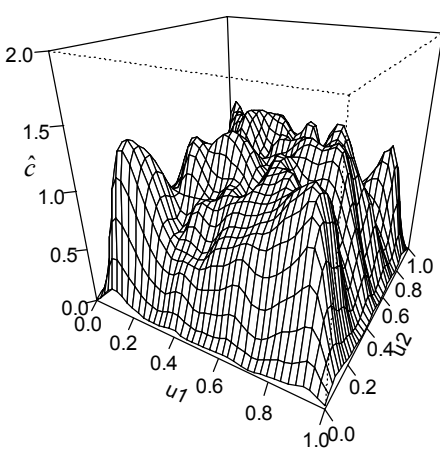

tau=3

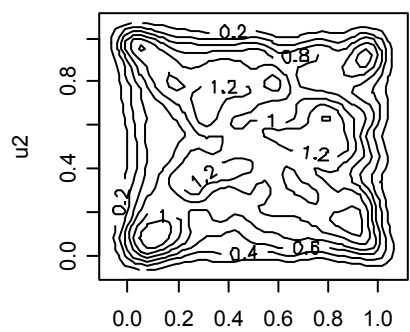

u1

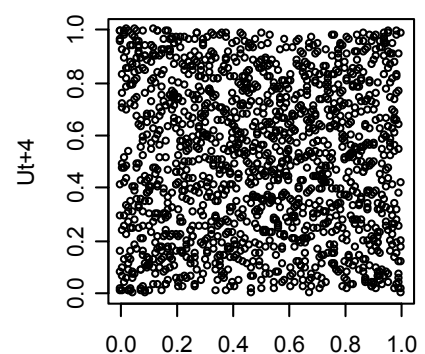

tau $=4$

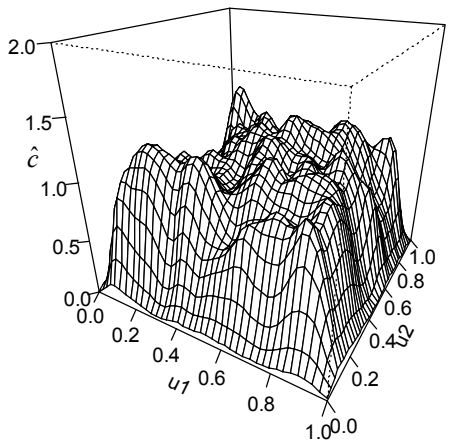

tau=4

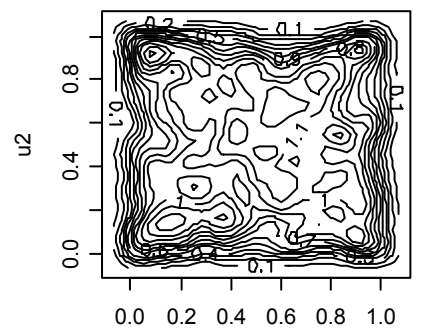

u1

Figura 2.21 - Gráficos de dispersão dos postos normalizados, da densidade cópula empírica suavizada e de suas respectivas curvas de nível (considerando os lags de 1 a 4), respectivamente, dos retornos diários da Petrobrás de 3/jan/95 a 27/dez/00. 


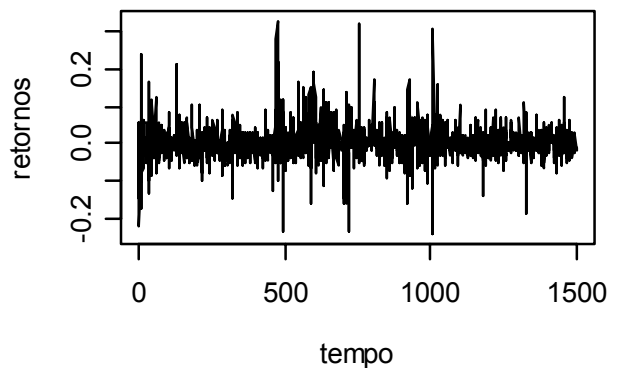

retornos

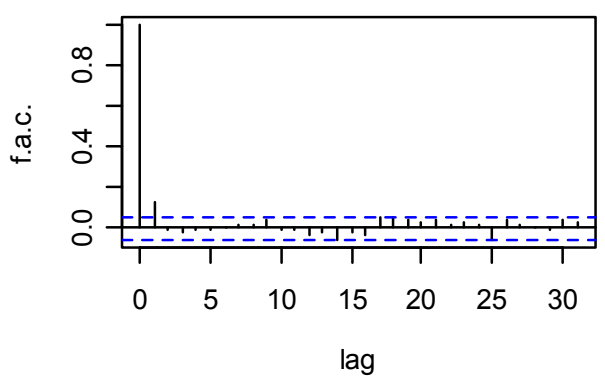

retornos $^{\wedge} 2$

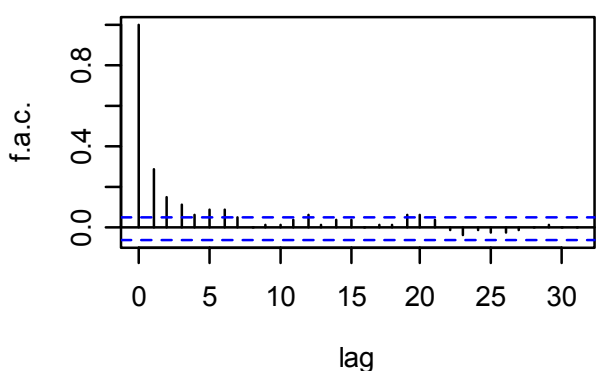

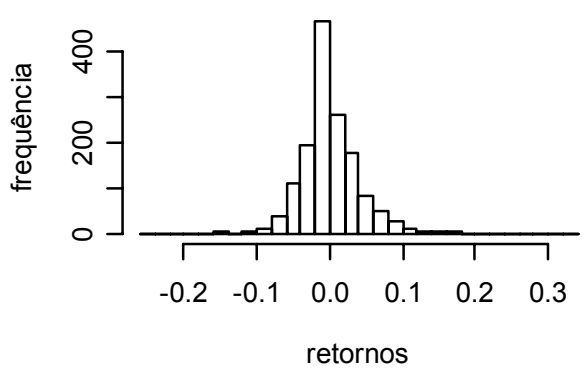

retornos

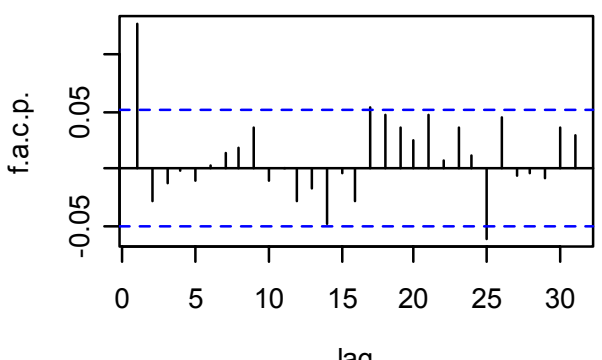

retornos^2

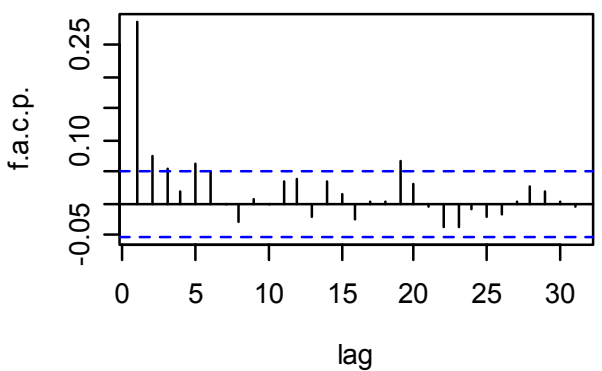

Figura 2.22 - Gráfico no tempo, histograma, f.a.c. e f.a.c.p. dos retornos, além da f.a.c. e f.a.c.p. dos retornos ao quadrado, respectivamente. Os retornos referem-se aos dos retornos diários do Banespa de 3/jan/95 a 27/dez/00. 


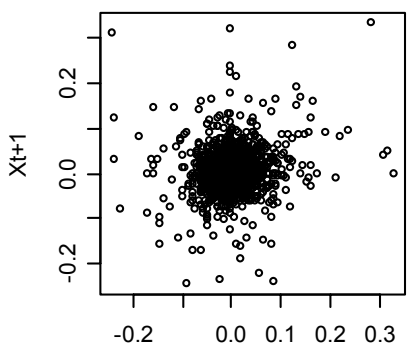

$\mathrm{Xt}$

tau $=$

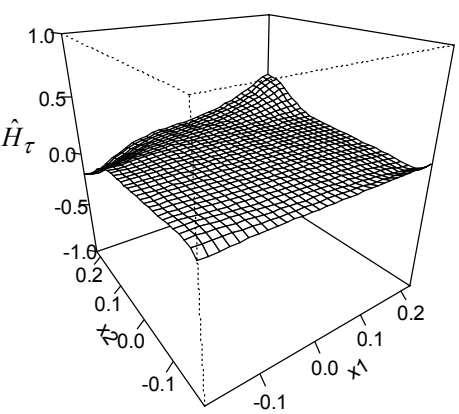

tau=1

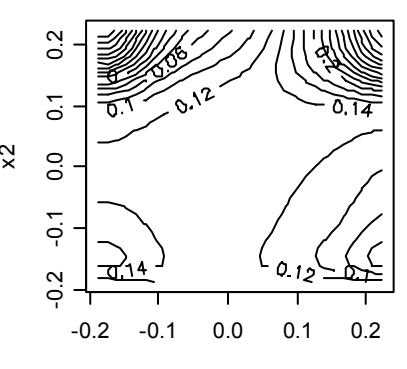

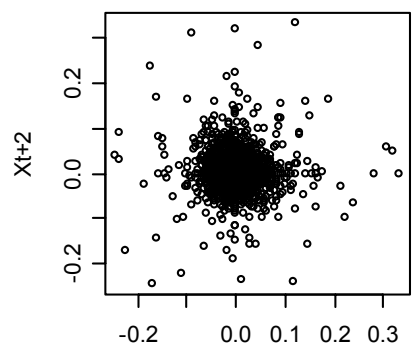

$\mathrm{xt}$

tau=2

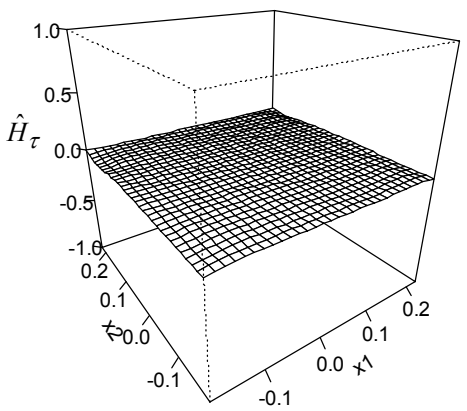

tau=2

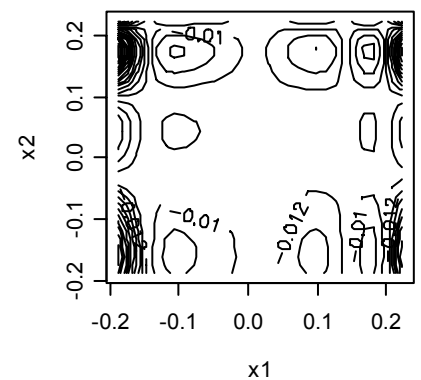

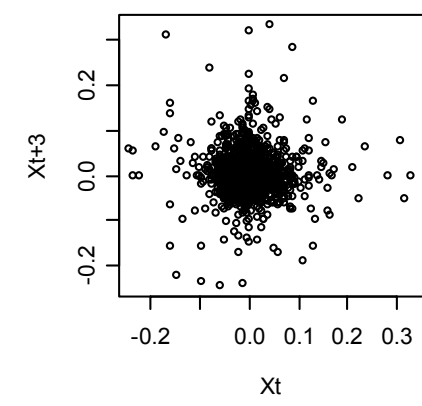

tau=3

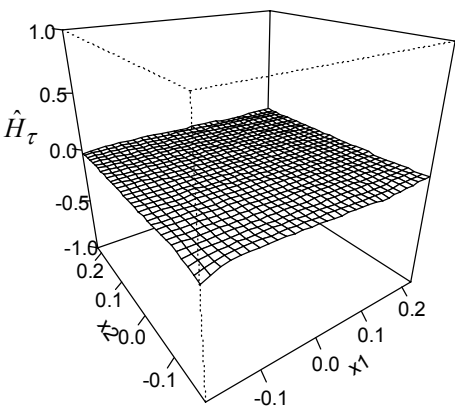

tau=3

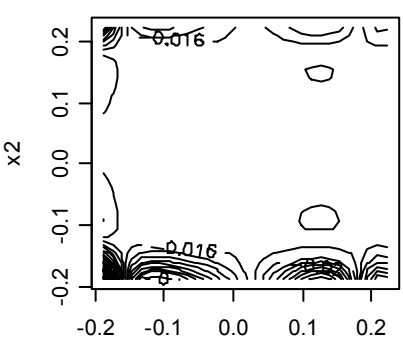

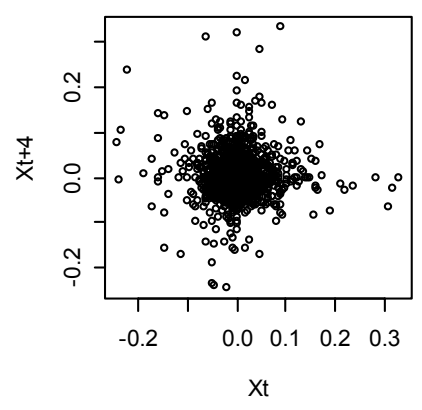

tau=4

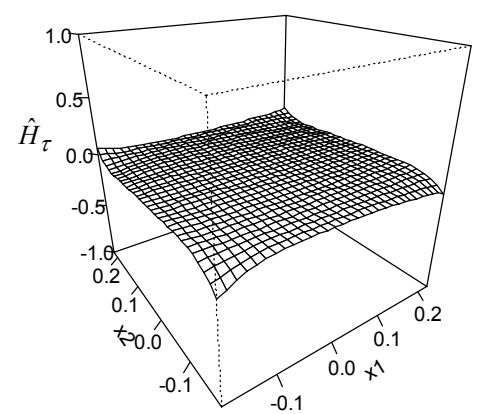

tau=4

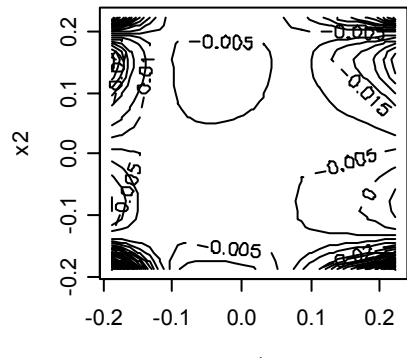

Figura 2.23 - Gráficos de dispersão, da f.a.c.l., e de curvas de nível da f.a.c.l. (considerando os lags de 1 a 4), respectivamente, dos retornos diários do Banespa de 3/jan/95 a 27/dez/00. 


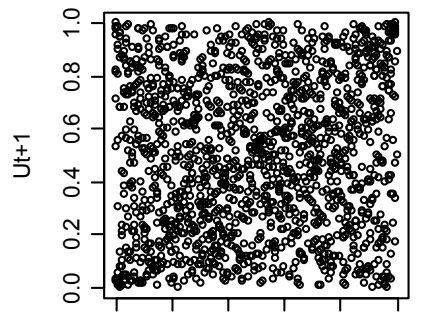

$\begin{array}{llllll}0.0 & 0.2 & 0.4 & 0.6 & 0.8 & 1.0\end{array}$

Ut

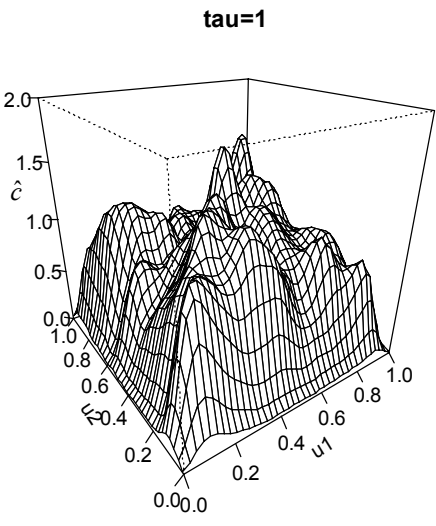

tau $=1$

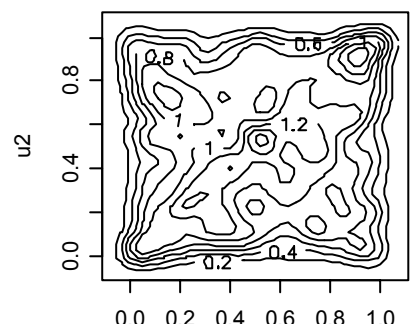

u1

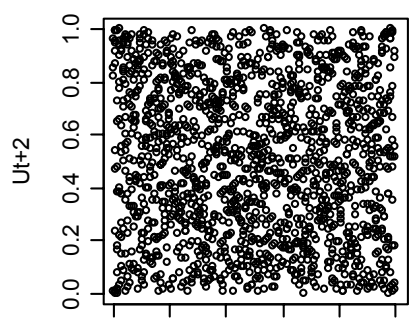

$\begin{array}{llllll}0.0 & 0.2 & 0.4 & 0.6 & 0.8 & 1.0\end{array}$

$\mathrm{Ut}$

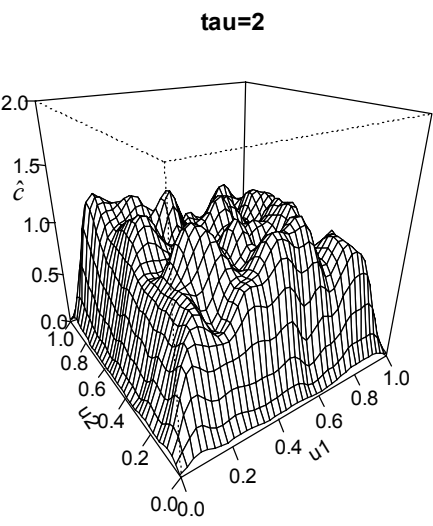

tau $=2$

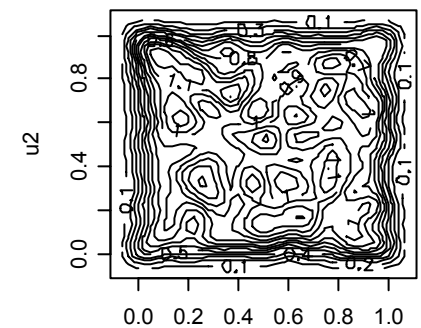

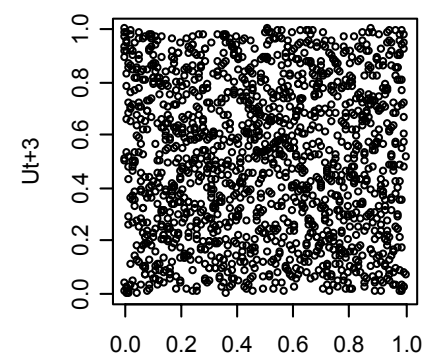

U

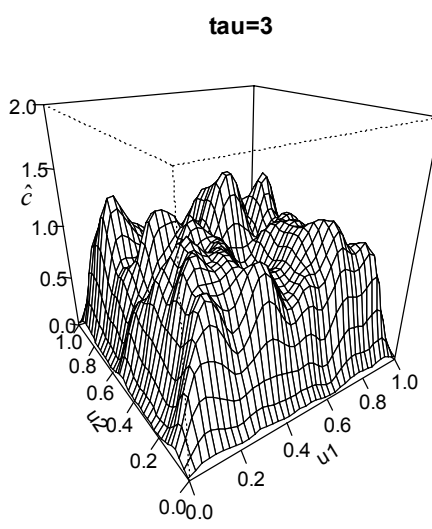

tau $=3$

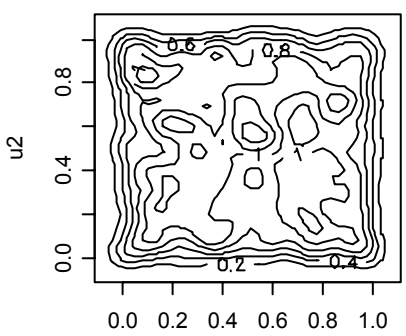

u1

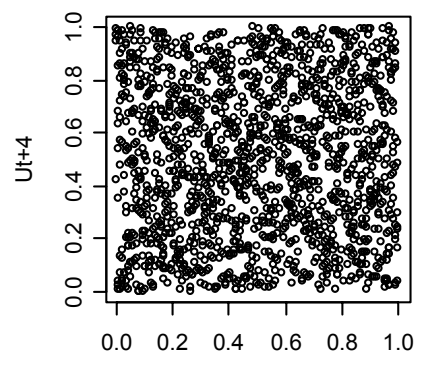

$\mathrm{Ut}$

tau $=4$

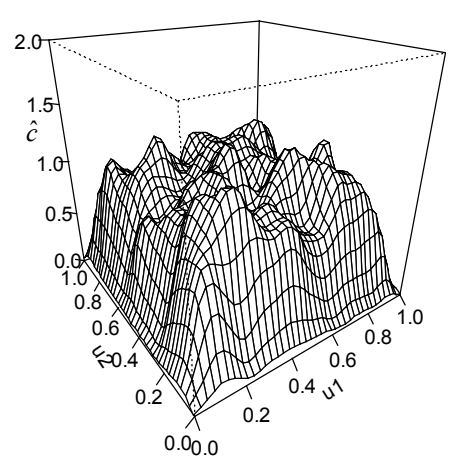

tau $=4$

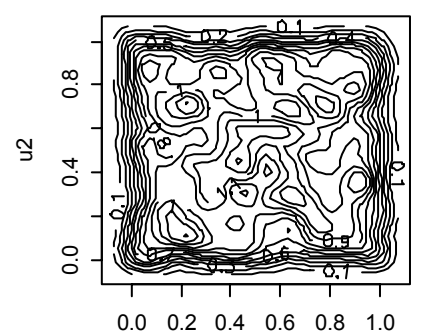

u1

Figura 2.24 - Gráficos de dispersão dos postos normalizados, da densidade cópula empírica suavizada e de suas respectivas curvas de nível (considerando os lags de 1 a 4), respectivamente, dos retornos diários do Banespa de 3/jan/95 a 27/dez/00. 


\subsection{Função de Correlação Cruzada Contemporânea Local}

Considere $\left\{\left(X_{t}, Y_{t}\right), t \in Z\right\}$ um processo a valores contínuos, estacionário estrito e com segundos momentos finitos. Então a distribuição conjunta e as distribuições marginais não dependem do tempo. Portanto, as médias $\left(\mu_{X}\right.$ e $\left.\mu_{Y}\right)$, as variâncias $\left(\sigma_{X}^{2}=\gamma_{X X}(0) \equiv \gamma_{X X}\right.$ e $\left.\sigma_{Y}^{2}=\gamma_{Y Y}(0) \equiv \gamma_{Y}\right)$ e a covariância cruzada contemporânea $\left(\gamma_{X Y}(0) \equiv \gamma_{X Y}\right)$ são constantes. Além disso, as esperanças condicionais $E\left[Y_{t} / X_{t}=x\right]$ e $E\left[X_{t} / Y_{t}=y\right]$ não dependem do tempo, as quais estão bem definidas para $f_{X}(x)>0 \mathrm{e}$ $f_{Y}(y)>0$, respectivamente.

Portanto, a medida de dependência local de Bairamov dada por (1.1), aqui denominada de função de correlação cruzada contemporânea local, não depende do tempo e pode ser escrita como

$$
\begin{aligned}
H_{0}(x, y)= & \frac{\rho_{X Y}+\left(\left(\mu_{X}-E\left[X_{t} / Y_{t}=y\right]\right) / \gamma_{X X}^{1 / 2}\right)\left(\left(\mu_{Y}-E\left[Y_{t} / X_{t}=x\right]\right) / \gamma_{Y Y}^{1 / 2}\right)}{\sqrt{1+\left(\left(\mu_{X}-E\left[X_{t} / Y_{t}=y\right]\right) / \gamma_{X X}^{1 / 2}\right)^{2}} \sqrt{1+\left(\left(\mu_{Y}-E\left[Y_{t} / X_{t}=x\right]\right) / \gamma_{Y Y}^{1 / 2}\right)^{2}}}, \\
& \forall(x, y) \in S, \forall t \in Z .
\end{aligned}
$$

As propriedades válidas para esta função local apresentam-se na proposição a seguir.

Proposição 2.2. Seja $\left\{\left(X_{t}, Y_{t}\right), t \in Z\right\}$ um processo estocástico a valores contínuos, estacionário estrito e com segundos momentos finitos. Então, a função de correlação cruzada contemporânea local, $H(x, y)$, satisfaz às seguintes propriedades:

(i) $\left|H_{0}(x, y)\right| \leq 1, \forall(x, y) \in S$;

(ii) Se $H_{0}(x, y)= \pm 1$ para algum $(x, y) \in S$, então $\rho_{X Y} \neq 0$;

(iii) Se $X_{t}=a Y_{t}+b$ quase certamente, então $H_{0}\left(X_{t}, Y_{t}\right)=\operatorname{sinal}(a)$ quase certamente, $\forall t \in Z$.

(iv) Se $\rho_{X Y}= \pm 1$, então $H\left(X_{t}, Y_{t}\right)= \pm 1$ quase certamente;

(v) Se $U_{t}=a+b X_{t}$ e $V_{t}=c+d Y_{t}$, com $b d \neq 0$, então $H_{U V}(u, v)=\operatorname{sinal}(b d) H_{X Y}(x, y)$, em que $u=a+b x$ e $v=c+d y, \forall(x, y) \in S, \forall t \in Z$. Notação: $H_{0}(x, y)=H_{X Y}(x, y)$;

(vi) Se $X_{t}$ e $Y_{t}$ são independentes, então $H_{0}(x, y)=0, \forall(x, y) \in S$;

(vii) Se $H_{0}(x, y)=0, \forall(x, y) \in S$, então $E\left[X_{t}\right]=E\left[X_{t} / Y_{t}=y\right]$ ou $E\left[Y_{t}\right]=E\left[Y_{t} / X_{t}=x\right], \forall(x, y) \in S$, e $\rho_{X Y}=0$;

(viii) $\mathrm{O}$ ponto $\left(x^{*}, y^{*}\right)$ satisfazendo $\mu_{X}-E\left[X_{t} / Y_{t}=y^{*}\right]=\mu_{Y}-E\left[Y_{t} / X_{t}=x^{*}\right]=0$ é ponto de sela de $H_{0}$, e neste ponto, $H_{0}\left(x^{*}, y^{*}\right)=\rho_{X Y}, \operatorname{com}\left(x^{*}, y^{*}\right) \in S ;$

(ix) $H_{0}\left(\mu_{X}, \mu_{Y}\right)=\rho_{X Y}$ se $\left(X_{t}, Y_{t}\right)$ tem distribuição normal; 
(x) De modo geral, $H_{0}(x, y) \neq H_{0}(y, x),(x, y) \in S$.

Essas propriedades possuem provas imediatas.

Agora, vamos considerar o caso em que o processo $\left(X_{t}, Y_{t}\right)$ é normal com média $\boldsymbol{\mu}=\left(\mu_{X}, \mu_{Y}\right)^{\prime} \mathrm{e}$ $\operatorname{vec}(\boldsymbol{\Gamma}(0))=\left(\gamma_{X X}, \gamma_{Y X}, \gamma_{X Y}, \gamma_{Y Y}\right)^{\prime}$, em que $\gamma_{X Y}=\gamma_{Y X}$. Então $E\left[X_{t} / Y_{t}=y\right]=\mu_{X}+\left(\gamma_{X Y} / \gamma_{Y Y}\right)\left(y-\mu_{Y}\right) \quad \mathrm{e}$ $E\left[Y_{t} / X_{t}=x\right]=\mu_{Y}+\left(\gamma_{X Y} / \gamma_{X X}\right)\left(x-\mu_{X}\right)$. Calculando a respectiva expressão da função de correlação cruzada contemporânea, temos:

$$
\begin{aligned}
H_{0}(x, y) & =\frac{\rho_{X Y}+\left(\left(\mu_{X}-E\left[X_{t+\tau} / Y_{t}=y\right]\right) /\left(\gamma_{X X}\right)^{1 / 2}\right)\left(\left(\mu_{Y}-E\left[Y_{t} / X_{t+\tau}=x\right]\right) /\left(\gamma_{Y Y}\right)^{1 / 2}\right)}{\sqrt{1+\left(\left(\mu_{X}-E\left[X_{t+\tau} / Y_{t}=y\right]\right) /\left(\gamma_{X X}\right)^{1 / 2}\right)^{2}} \sqrt{1+\left(\left(\mu_{Y}-E\left[Y_{t} / X_{t+\tau}=x\right]\right) /\left(\gamma_{Y Y}\right)^{1 / 2}\right)^{2}}}, \\
& =\frac{\rho_{X Y}+\left(\gamma_{X Y}^{2} /\left(\gamma_{X X}^{3 / 2} \gamma_{Y Y}^{3 / 2}\right)\right)\left(y-\mu_{Y}\right)\left(x-\mu_{X}\right)}{\sqrt{1+\left(\gamma_{X Y}^{2} /\left(\gamma_{Y Y}^{2} \gamma_{X X}\right)\right)\left(y-\mu_{Y}\right)^{2}} \sqrt{\left.1+\left(\gamma_{X Y}^{2} / \gamma_{X X}^{2} \gamma_{Y Y}\right)\right)\left(x-\mu_{X}\right)^{2}}}
\end{aligned}
$$

ou seja

$$
H_{0}(x, y)=\frac{\rho_{X Y}\left(1+\left(\rho_{X Y} /\left(\gamma_{X X} \gamma_{Y Y}\right)^{1 / 2}\right)\left(x-\mu_{X}\right)\left(y-\mu_{Y}\right)\right)}{\sqrt{1+\left(\rho_{X Y}^{2} / \gamma_{Y Y}\right)\left(y-\mu_{Y}\right)^{2}} \sqrt{1+\left(\rho_{X Y}^{2} / \gamma_{X X}\right)\left(x-\mu_{X}\right)^{2}}}, \quad \forall(x, y) \in S, \forall t \in Z .
$$

Como estimador desta função local, propomos o mesmo tipo de estimador da função de autocorrelação local (seção 2.4). Ou seja, observados $\left(\left(X_{1}, Y_{1}\right), \ldots,\left(X_{T}, Y_{T}\right)\right)$, então

$$
\hat{H}_{0}(x, y)=\frac{r_{X Y}+\left((\bar{X}-\hat{m}(y ; \tau)) / c_{X X}^{1 / 2}\right)\left((\bar{Y}-\hat{m}(x ; \tau)) / c_{Y Y}^{1 / 2}\right)}{\sqrt{1+\left((\bar{X}-\hat{m}(y ; \tau)) / c_{X X}^{1 / 2}\right)^{2}} \sqrt{1+\left((\bar{Y}-\hat{m}(x ; \tau)) / c_{Y Y}^{1 / 2}\right)^{2}}}
$$

em que

$$
\begin{aligned}
& \bar{X}=\frac{1}{T} \sum_{t=1}^{T} X_{t} \\
& \bar{Y}=\frac{1}{T} \sum_{t=1}^{T} Y_{t} \\
& c_{X X}=\frac{1}{T} \sum_{t=1}^{T}\left(X_{t}-\bar{X}\right)^{2}, \\
& c_{Y Y}=\frac{1}{T} \sum_{t=1}^{T}\left(Y_{t}-\bar{Y}\right)^{2}, \\
& r_{X Y}=\frac{c_{X Y}}{\sqrt{c_{X X} c_{Y Y}}}, \text { em que } c_{X Y}=\frac{1}{T} \sum_{t=1}^{T}\left(X_{t}-\bar{X}\right)\left(Y_{t}-\bar{Y}\right), \\
& \hat{m}(y ; \tau)=\sum_{t=1}^{T} X_{t} K_{2}\left(\frac{y-Y_{t}}{h_{2, T}}\right) / \sum_{t=1}^{T} K_{2}\left(\frac{y-Y_{t}}{h_{2, T}}\right)
\end{aligned}
$$




$$
\hat{m}(x ; \tau)=\sum_{t=1}^{T} Y_{t} K_{1}\left(\frac{x-X_{t}}{h_{1, T}}\right) / \sum_{t=1}^{T} K_{1}\left(\frac{x-X_{t}}{h_{1, T}}\right),
$$

com $K_{i}$ e $h_{i, T}, i=1,2$, como antes, e $(x, y) \in G$.

A seguir, apresenta-se o teorema de consistência do estimador da função de correlação cruzada contemporânea local. Antes, porém, vejamos as condições de regularidade a serem consideradas.

(C1) $X_{t}=\mu_{X}+\sum_{j=-\infty}^{+\infty} \alpha_{j} e_{t-j} \quad$, em que $\quad e_{t} \sim i i d\left(0, \sigma_{e}^{2}\right), \quad E\left[e_{t}^{4}\right]=\eta \sigma_{e}^{4}, \quad \sum_{j=-\infty}^{+\infty}\left|\alpha_{j}\right|<\infty \quad$ e $\sum_{h=-\infty}^{+\infty}\left|\gamma_{X X}(h)\right|<\infty$. Expressões similares para $Y_{t} ;$

(C2) os parâmetros de suavização $h_{i, T}>0, i=1,2$, são tais que $h_{i, T} \rightarrow 0$ e $T h_{i, T} \rightarrow \infty$, quando $T \rightarrow \infty$;

(C3) os kernels $K_{i}, i=1,2$, são funções densidade limitadas, simétricas (em torno do zero) e tal que

$$
\lim _{z \rightarrow \infty} z K_{i}(z)=0 \text { e } \int z^{2} K_{i}(z) d z<+\infty ;
$$

(C4) $K_{i}, \quad i=1,2$, são funções contínuas Lipschitzianas de ordem $\gamma_{i}$ em $\mathfrak{R}$, ou seja, $\left|K_{i}\left(z_{1}\right)-K_{i}\left(z_{2}\right)\right| \leq c_{i}\left|z_{1}-z_{2}\right|^{\gamma_{i}}, \operatorname{com} z_{i} \in \mathfrak{R}, \gamma_{i}>0, i=1,2$;

(C5) $\left(X_{t}, Y_{t}\right)$ é um processo $\alpha$-mixing, com coeficiente apresentando decaimento geométrico, ou seja, $\exists u \in] 0 ; \infty\left[\right.$ e $\exists v \in\left[0 ; 1\left[\right.\right.$ tal que $\alpha(k) \leq u v^{k}, k \geq 1 ;$

(C6) $r_{Y}$ e $f_{X}$ são funções duas vezes continuamente deriváveis e com valores em $\mathfrak{R}$, e tais que

$$
\begin{aligned}
& \left\|f_{X}\right\|_{\infty}=\inf \left\{a: P\left(f_{X}>a\right)=0\right\} \leq b \text { e }\left\|f_{X}^{(2)}\right\|_{\infty} \leq b, \\
& \left\|r_{Y}\right\|_{\infty}=\inf \left\{a: P\left(r_{Y}>a\right)=0\right\} \leq b \text { e }\left\|r_{Y}^{(2)}\right\|_{\infty} \leq b,
\end{aligned}
$$

para algum $b$, em que $r_{Y}(x)=\int y f(x, y) d y$ é o numerador de $E\left[Y_{t} / X_{t}=x\right], \forall t \in Z$;

(C7) $r_{X}$ e $f_{Y}$ são funções duas vezes continuamente deriváveis e com valores em $\mathfrak{R}$, e tais que

$$
\left\|f_{Y}\right\|_{\infty} \leq c,\left\|f_{Y}^{(2)}\right\|_{\infty} \leq c,\left\|r_{X}\right\|_{\infty} \leq c \text { e }\left\|r_{X}^{(2)}\right\|_{\infty} \leq c
$$

para algum $c$, em que $r_{X}(y)=\int x f(x, y) d x$ é o numerador de $E\left[X_{t} / Y_{t}=y\right], \forall t \in Z$;

(C8) $E\left[\exp \left\{d\left|X_{t}\right|^{s_{1}}\right\}\right]<+\infty$, para algum $d>0$, e algum $s_{1}>0, \forall t \in Z$;

(C9) $E\left[\exp \left\{a\left|Y_{t}\right|^{s_{2}}\right\}\right]<+\infty$, para algum $a>0$ e algum $s_{2}>0, \forall t \in Z$;

(C10) $\frac{T h_{i, T}}{(\log T)^{2+1 / s_{i}}} \rightarrow+\infty, T \rightarrow \infty, s_{i}>0, i=1,2$;

(C11) $S$ é um conjunto compacto tal que $\inf _{x \in S} f_{X}(x)>0 \quad$ e $\quad \inf _{y \in S} f_{Y}(y)>0$; 
(C12) $h_{i, T} \cong\left(\frac{(\log T)^{2-\left(1 / s_{i}\right)}}{T}\right)^{1 / 5}, s_{i}>0, i=1,2$.

Teorema 2.2. Seja $\left\{\left(X_{t}, Y_{t}\right), t \in Z\right\}$ um processo estacionário estrito e com segundos momentos finitos. Satisfeitas as condições $(\mathrm{C} 1)$ a (C13) anteriores, então

$$
\hat{H}_{0}(x, y) \underset{T \rightarrow \infty}{\stackrel{P}{\longrightarrow}} H_{0}(x, y) \text {, para cada }(x, y) \in S \text { fixados. }
$$

prova:

Por (C1) e pelo Corolário 6.1.1.2 de Fuller (1976), temos que $\bar{X}$ e $\bar{Y}$ são consistentes para $\mu_{X}$ e $\mu_{Y}$, respectivamente, portanto,

$$
\bar{X} \underset{T \rightarrow \infty}{\stackrel{P}{\longrightarrow}} \mu_{X} \text { e } \bar{Y} \underset{T \rightarrow \infty}{\stackrel{P}{\longrightarrow}} \mu_{Y} .
$$

Por (C1) e pelo Teorema 6.2.2 de Fuller (1976), temos que $c_{X X}$ é consistente para $\gamma_{X X}$, e $c_{Y Y}$ é consistente para $\gamma_{Y Y}$, respectivamente. Ou seja,

$$
c_{X X} \underset{T \rightarrow \infty}{\stackrel{P}{\longrightarrow}} \gamma_{X X} \text { e } \quad c_{Y Y} \underset{T \rightarrow \infty}{\stackrel{P}{\longrightarrow}} \gamma_{Y Y} .
$$

Segundo Fuller (1976), satisfeitas as condições das f.a.c.v.'s serem absolutamente somáveis em (C1), então a função de covariância cruzada contemporânea é assintoticamente não viesada (página 263) e a variância do estimador vai para zero quando $T \rightarrow \infty$ (Teorema 6.5.1). Portanto,

$$
c_{X Y} \underset{T \rightarrow \infty}{\stackrel{P}{\longrightarrow}} \gamma_{X Y} .
$$

Pelo Teorema 3.2 de Bosq (1998), sendo $\left\{\left(X_{t}, Y_{t}\right)\right\}$ um processo estacionário estrito com as condições (C2) a (C12) satisfeitas, então $\hat{m}(x ; \tau) \stackrel{q . c .}{\longrightarrow} m(x ; \tau)$ e $\hat{m}(y ; \tau) \stackrel{q . c .}{\longrightarrow} m(y ; \tau)$, ou seja

$$
\hat{m}(x ; \tau) \underset{T \rightarrow \infty}{\stackrel{P}{\longrightarrow}} m(x ; \tau) \text { e } \hat{m}(y ; \tau) \underset{T \rightarrow \infty}{\stackrel{P}{\longrightarrow}} m(y ; \tau),
$$

para $h_{i, T} \rightarrow 0, T h_{i, T} \rightarrow \infty$, ambos quando $T \rightarrow \infty$, e para cada $x, y \in S$ fixados.

Considere

$$
\begin{aligned}
& \hat{\boldsymbol{\theta}}=\left(\bar{X}, \bar{Y}, c_{X X}, c_{Y Y}, c_{X Y}, \hat{m}(x ; \tau), \hat{m}(y ; \tau)\right)^{\prime}=\left(\hat{\theta}_{1}, \hat{\theta}_{2}, \hat{\theta}_{3}, \hat{\theta}_{4}, \hat{\theta}_{5}, \hat{\theta}_{6}, \hat{\theta}_{7}\right)^{\prime}, \\
& \boldsymbol{\theta}=\left(\mu_{X}, \mu_{Y}, \gamma_{X X}, \gamma_{Y Y}, \gamma_{X Y}, m(x ; \tau), m(y ; \tau)\right)^{\prime}=\left(\theta_{1}, \theta_{2}, \theta_{3}, \theta_{4}, \theta_{5}, \theta_{6}, \theta_{7}\right)^{\prime} .
\end{aligned}
$$

De modo similar ao Teorema 2.1, utilizando o Lema 5.1.3 e o Teorema 5.1.4 de Fuller (1976), temos que uma função contínua (com denominador não nulo) de estimadores que convergem em probabilidade para o verdadeiro parâmetro, também converge em probabilidade para a função contínua dos parâmetros. Portanto, $\hat{H}_{0}(x, y) \stackrel{P}{\longrightarrow} H_{0}(x, y)$, para $h_{i, T} \rightarrow 0$ e $T h_{i, T} \rightarrow \infty, i=1,2$, ambos quando $T \rightarrow \infty$, para cada $x, y \in S$ fixados. 


\subsubsection{Simulações}

Avaliamos a função de correlação cruzada contemporânea local utilizando simulações da função teórica e estimada dos modelos bivariados VAR(1) e VMA(1) estacionários, calculando-se as estatísticas usuais do estimador. Para isto, utilizamos 200 réplicas de Monte Carlo com correlação contemporânea 0,70 e nula, para séries de tamanho 50,100, 200 e 500. Novamente, utilizamos grade de $31 \times 31$ pontos, $90 \%$ dos dados centrais, kernel Gaussiano e largura segundo a condição (C12) do Teorema 2.2.

Inicialmente, consideramos o modelo autoregressivo vetorial estacionário de ordem um dado por

$$
\mathbf{Z}_{t}=\boldsymbol{\Phi}_{0}+\boldsymbol{\Phi}_{1} \mathbf{Z}_{t-1}+\boldsymbol{\varepsilon}_{t},
$$

em que $\quad \mathbf{Z}_{t}=\left(X_{t}, Y_{t}\right), \quad \boldsymbol{\Phi}_{0}=(1,1)^{\prime}, \quad \operatorname{vec}\left(\boldsymbol{\Phi}_{1}\right)=(0,25 ; 0,2 ; 0,2 ; 0,75)^{\prime} \quad$ e $\quad \boldsymbol{\varepsilon}_{t} \sim N(0, \boldsymbol{\Sigma}), \quad$ com $\operatorname{vec}(\boldsymbol{\Sigma})=(0,75 ; 0,5 ; 0,5 ; 1,25)^{\prime}$, ou seja, $\mathbf{Z}_{t}$ tem componentes dependentes. Portanto, os parâmetros da distribuição Gaussiana estacionária são $\boldsymbol{\mu}=(3,05 ; 6,44)^{\prime}$ e $\operatorname{vec}(\boldsymbol{\Gamma}(0))=(1,13 ; 1,49 ; 1,49 ; 3,99)^{\prime}$ (correlação 0,70$)$. Então, a função teórica foi construída com base nesta distribuição, e a função estimada foi elaborada através de 200 réplicas de Monte Carlo com séries de tamanho 500, observadas desse modelo VAR(1). Na Figura 2.25, encontram-se os gráficos: (a) função de correlação cruzada contemporânea local teórica, (b) respectivas curvas de nível, (c) função local estimada e (d) curvas de nível correspondentes. Comparando os dois primeiros gráficos com os dois últimos, verificamos a ocorrência de uma pequena superestimação da medida nos extremos da diagonal secundária. Para alguns pontos de grade da diagonal principal e secundária, podemos verificar na Tabela 2.11 o viés, o erro quadrático médio e o $p$-valor do teste de normalidade de Jarque Bera que rejeitou a normalidade do estimador na diagonal principal e em alguns pontos da diagonal secundária. Além disso, nas Figuras 2.26 e 2.27, apresentam-se os histogramas das funções estimadas na diagonal principal e na diagonal secundária, respectivamente, os quais não indicam forte afastamento da normalidade nos pontos mais centrais.

Repetimos estas simulações para séries de tamanho 50, 100 e 200, cujos resultados principais apresentam-se na Figura B.4 do Apêndice B. Comparando-se as quatro simulações, verificamos que os gráficos das curvas de nível da amostra menor são similares às da amostra maior, e que aumentando-se o tamanho da série, diminuem o viés e o erro quadrático médio, e com $\alpha=5 \%$, a rejeição da normalidade permanece praticamente inalterada na diagonal principal e diminui na diagonal secundária para as duas amostras maiores.

Agora, vamos avaliar o modelo VAR(1) com as componentes de $\mathbf{Z}_{t}$ independentes, no caso, com $\boldsymbol{\Phi}_{0}=(1,1)^{\prime}, \quad \operatorname{vec}\left(\boldsymbol{\Phi}_{1}\right)=(0,25 ; 0 ; 0 ; 0,75)^{\prime} \quad$ e $\operatorname{vec}(\boldsymbol{\Sigma})=(0,75 ; 0 ; 0 ; 1,25)^{\prime}$. Então, os parâmetros da distribuição Gaussiana estacionária são $\boldsymbol{\mu}=(1,33 ; 4)^{\prime}$ e $\operatorname{vec}(\boldsymbol{\Gamma}(0))=(0,8 ; 0 ; 0 ; 2,86)^{\prime}$ (correlação nula). Novamente, para avaliarmos o estimador, construímos a função teórica utilizando esta distribuição e geramos 200 séries de tamanho 500 a partir deste modelo. Na Figura 2.28 temos os gráficos (a) e (c) da função de correlação cruzada contemporânea local teórica e estimada, respectivamente, além de seus correspondentes gráfícos de curvas de nível em (b) e (d), os quais indicam comportamento muito similar. Na Tabela 2.12, apresentam-se 
as estatísticas básicas do estimador. Cabe ressaltar que a normalidade não foi rejeitada, exceto num ponto extremo da diagonal secundária. Nas figuras 2.29 e 2.30, temos os respectivos histogramas.

Avaliando simulações similares, mas agora com séries de tamanho 50, 100 e 200 (veja Figura B.5 do Apêndice B), verificamos a grande similaridade entre os gráficos das curvas de nível. Observamos também que, conforme aumenta o tamanho da série, o viés (em módulo) geralmente diminui, o erro quadrático médio diminui, e a rejeição da normalidade diminui a partir de amostras com tamanho 500.

Comparando as simulações de correlação contemporânea 0,70 com as de correlação contemporânea nula (para os quatro tamanhos de série avaliados), verificamos que o viés, o erro quadrático médio e a rejeição da normalidade diminuem com a redução da correlação.

O modelo VMA(1) estacionário considerado inicialmente foi

$$
\mathbf{Z}_{t}=\boldsymbol{\Theta}_{0}-\boldsymbol{\Theta}_{1} \boldsymbol{\varepsilon}_{t-1}+\boldsymbol{\varepsilon}_{t},
$$

em que $\quad \mathbf{Z}_{t}=\left(X_{t}, Y_{t}\right)^{\prime}, \quad \boldsymbol{\Theta}_{0}=(0,4 ;-1,7)^{\prime}, \quad \operatorname{vec}\left(\boldsymbol{\Theta}_{1}\right)=(0,8 ;-0,4 ; 0,7 ; 0,6)^{\prime} \quad$ e $\quad \boldsymbol{\varepsilon}_{t} \sim N(\mathbf{0}, \boldsymbol{\Sigma}) \quad$ com $\operatorname{vec}(\boldsymbol{\Sigma})=(0,75 ; 0,92 ; 0,92 ; 1,24)^{\prime}$. Então o processo tem distribuição Gaussiana estacionária com parâmetros $\boldsymbol{\mu}=(0,4 ;-1,7)^{\prime}$ e $\operatorname{vec}(\boldsymbol{\Gamma}(0))=(2,87 ; 1,39 ; 1,39 ; 1,37)^{\prime}$, resultando em correlação 0,70 . Para obtermos a função de correlação cruzada contemporânea local estimada, utilizamos 200 séries de tamanho 500 observadas deste modelo. Na Figura 2.31, podemos observar uma pequena superestimação da medida nos extremos da diagonal secundária, sendo que este comportamento pode ser confirmado numericamente através da Tabela 2.13, a qual também mostra a não rejeição da normalidade do estimador (exceto num ponto). Nas figuras 2.32 e 2.33, observamos os histogramas do estimador os quais indicam distribuição aproximadamente normal.

Na Figura B.6 do apêndice B, encontram-se os resultados de simulações similares, mas com séries de tamanho 50, 100 e 200. Comparando estas simulações com a simulação de séries de tamanho 500, podemos observar a semelhança dos gráficos das curvas de nível para $T=50$ e $T=500$. Além disso, com o aumento do tamanho da série, o viés e o erro quadrático médio diminuem, e a rejeição da normalidade diminui a partir de amostras de tamanho 500 .

Também, o modelo VMA(1) estacionário com componentes independentes foi avaliado, no caso, com $\boldsymbol{\Theta}_{0}=(0,4 ;-1,7)^{\prime}, \quad \operatorname{vec}\left(\boldsymbol{\Theta}_{1}\right)=(0,8 ; 0 ; 0 ; 0,6)^{\prime}$ e $\boldsymbol{\varepsilon}_{t} \sim N(\mathbf{0}, \boldsymbol{\Sigma}) \quad \operatorname{com} \quad \operatorname{vec}(\boldsymbol{\Sigma})=(4 ; 0 ; 0 ; 2)^{\prime}$. Então o processo tem distribuição Gaussiana estacionária com parâmetros $\boldsymbol{\mu}=(0,4 ;-1,7)^{\prime}$ e $\operatorname{vec}(\boldsymbol{\Gamma}(0))=(6,56 ; 0 ; 0 ; 2,72)^{\prime}$, resultando em correlação nula. Utilizando 200 séries de tamanho 500, estimamos a função de correlação cruzada contemporânea local, a qual apresenta-se praticamente nula e constante, conforme podemos observar nos gráficos (c) e (d) da Figura 2.34, a qual também contém os gráficos (a) e (b) da função local teórica. Na Tabela 2.14 podemos observar que o viés e o erro quadrático médio do estimador são praticamente nulos, e que há rejeição da normalidade nos pontos mais extremos das diagonais. Este comportamento pode ser observado através dos histogramas, os quais apresentam-se nas figuras 2.35 e 2.36 .

As simulações foram refeitas com séries de tamanhos 50, 100 e 200, cujos resultados apresentam-se na Figura B.7 do Apêndice B. Verificamos que com o aumento do tamanho da série, o viés e o erro quadrático médio diminuem, mas a rejeição da normalidade permanece similar nos pontos mais extremos das diagonais. Além disso, os gráficos das curvas de nível da amostra menor e da amostra maior, são muito semelhantes. 
Com referência ao modelo VMA(1), ao compararmos as quatro simulações de correlação contemporânea 0,70 com as de correlação nula, verificamos que nesta última, o viés é menor, o erro quadrático médio da diagonal principal é maior e da diagonal secundária é menor, e a rejeição da normalidade diminui nos extremos das diagonais, exceto com $T=500$ em que a rejeição passa a ser maior.

Comparando o modelo $\operatorname{VAR}(1)$ com o $\operatorname{VMA}(1)$, verificamos que este último modelo geralmente apresenta viés e erro quadrático médio menor, embora para amostras de tamanho 500 estas estimativas apresentam-se similares. Também, para séries de tamanho 500, o modelo VMA(1) apresenta menor rejeição da normalidade quando a correlação contemporânea é 0,70 , e maior rejeição quando a correlação contemporânea é nula. 


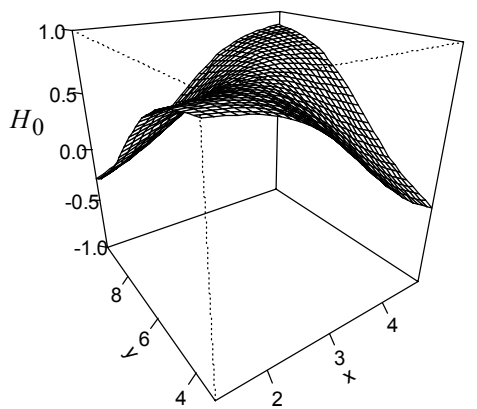

(a)

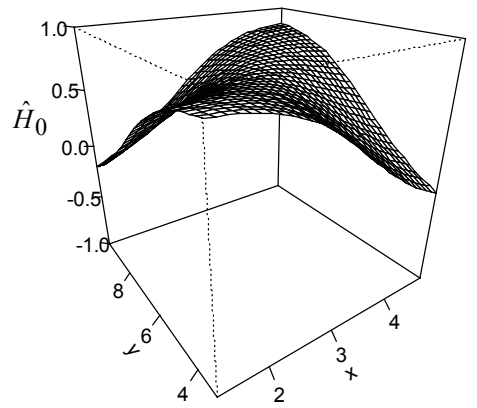

(c)

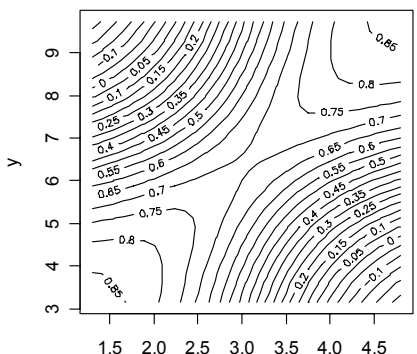

(b)

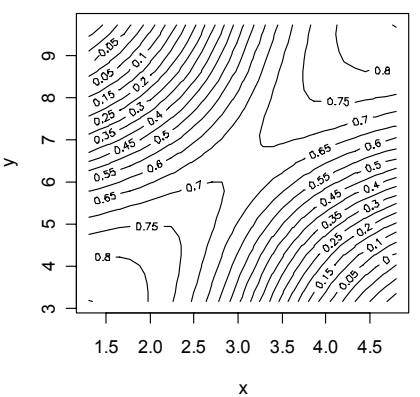

(d)

Figura 2.25 - Considerando a função de correlação cruzada contemporânea local e o modelo VAR(1) com média $(3,05 ; 6,44)^{\prime}, \operatorname{vec}(\Gamma(0))=(1,13 ; 1,49 ; 1,49 ; 3,99)^{\prime}$ (correlação 0,70$)$ e inovações Gaussianas, temos: (a) gráfico da função teórica, (b) gráfico das respectivas curvas de nível, (c) gráfico da função estimada e (d) gráfico das respectivas curvas de nível, estes considerando 200 séries de tamanho 500.

Tabela 2.11 - Viés, erro quadrático médio e $p$-valor do teste de normalidade de Jarque Bera, para sete pontos de grade da diagonal principal e secundária da função de correlação cruzada contemporânea local, considerando o modelo VAR(1) com média $(3,05 ; 6,44)^{\prime}$, $\operatorname{vec}(\Gamma(0))=(1,13 ; 1,49 ; 1,49 ; 3,99)^{\prime}$ (correlação 0,70) e inovações Gaussianas com 200 séries de tamanho 500.

\begin{tabular}{|l|l|l|l|l|r|r|r|}
\hline Grade X & $\mathbf{1 , 3 0}$ & $\mathbf{1 , 8 9}$ & $\mathbf{2 , 4 7}$ & $\mathbf{3 , 0 5}$ & $\mathbf{3 , 6 3}$ & $\mathbf{4 , 2 2}$ & $\mathbf{4 , 8 0}$ \\
\hline Grade Y & $\mathbf{3 , 1 6}$ & $\mathbf{4 , 2 5}$ & $\mathbf{5 , 3 5}$ & $\mathbf{6 , 4 4}$ & $\mathbf{7 , 5 4}$ & $\mathbf{8 , 6 3}$ & $\mathbf{9 , 7 2}$ \\
\hline Viés $^{(1)}$ & $-0,022$ & $-0,023$ & $-0,017$ & $-0,010$ & $-0,015$ & $-0,021$ & $-0,022$ \\
\hline Viés $^{(2)}$ & 0,096 & 0,081 & 0,029 & $-0,010$ & 0,028 & 0,076 & 0,085 \\
\hline EQM $^{(1)}$ & 0,001 & 0,002 & 0,002 & 0,001 & 0,001 & 0,001 & 0,001 \\
\hline EQM $^{(2)}$ & 0,014 & 0,011 & 0,004 & 0,001 & 0,005 & 0,011 & 0,012 \\
\hline P-valor $^{(1)}$ & 0,000 & 0,000 & 0,000 & 0,000 & 0,001 & 0,005 & 0,000 \\
\hline -valor $^{(2)}$ & 0,130 & 0,702 & 0,912 & 0,000 & 0,239 & 0,027 & 0,000 \\
\hline
\end{tabular}

${ }^{(1)}$ diagonal principal ; ${ }^{(2)}$ diagonal secundária. 
$1.3,3.16$

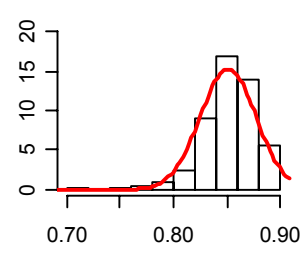

$3.63,7.54$

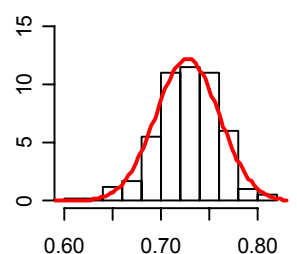

$1.89,4.25$

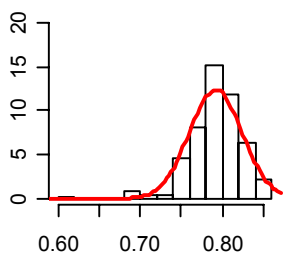

4.22, 8.63

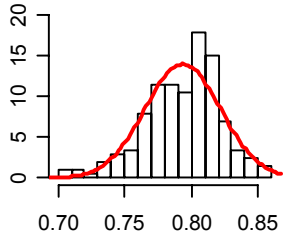

$2.47,5.35$

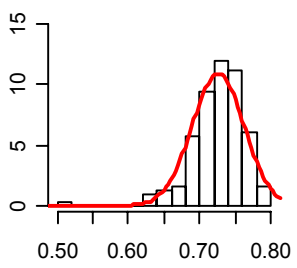

4.8, 9.72

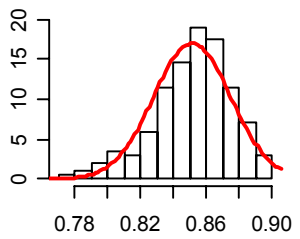

Figura 2.26 - Histogramas em pontos de grade da diagonal principal do estimador da função de correlação cruzada contemporânea local, considerando 200 séries de tamanho 500, observadas do modelo VAR(1) com média $(3,05 ; 6,44)^{\prime}, \operatorname{vec}(\Gamma(0))=(1,13 ; 1,49 ; 1,49 ; 3,99)^{\prime}$ (correlação 0,70$)$ e inovações Gaussianas.

$1.3,9.72$

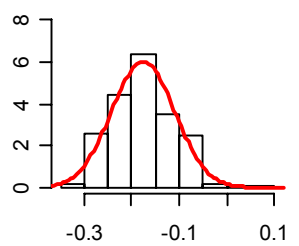

$3.63,5.35$

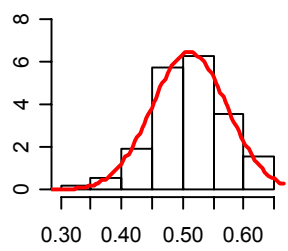

$1.89,8.63$

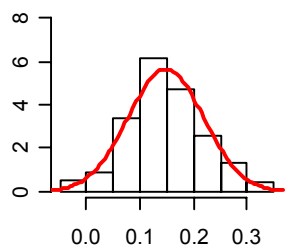

$4.22,4.25$

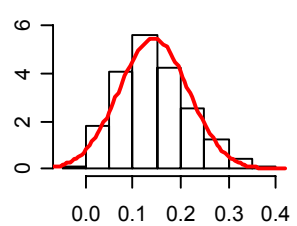

2.47, 7.54

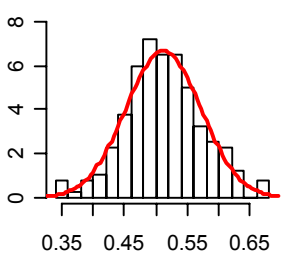

4.8, 3.16

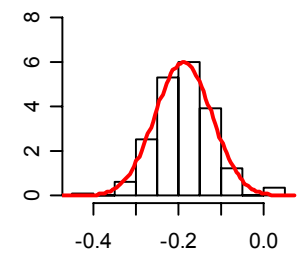

Figura 2.27 - Histogramas em pontos de grade da diagonal secundária do estimador da função de correlação cruzada contemporânea local, considerando 200 séries de tamanho 500, observadas do modelo VAR(1) com média $(3,05 ; 6,44)^{\prime}, \operatorname{vec}(\Gamma(0))=(1,13 ; 1,49 ; 1,49 ; 3,99)^{\prime}$ (correlação 0,70$)$ e inovações Gaussianas. 


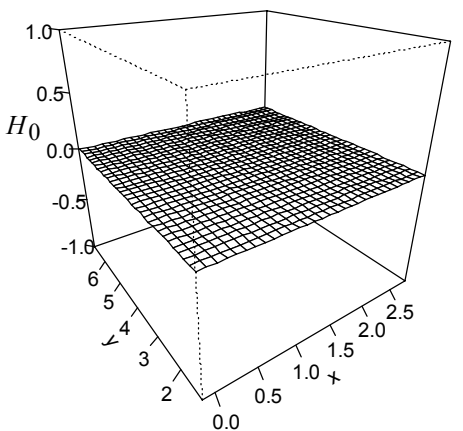

(a)

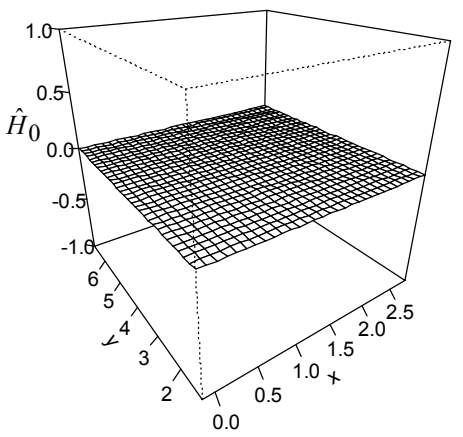

(c)

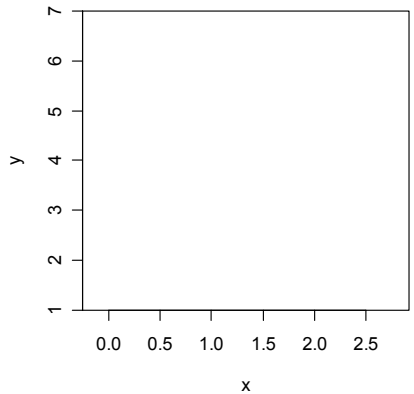

(b)

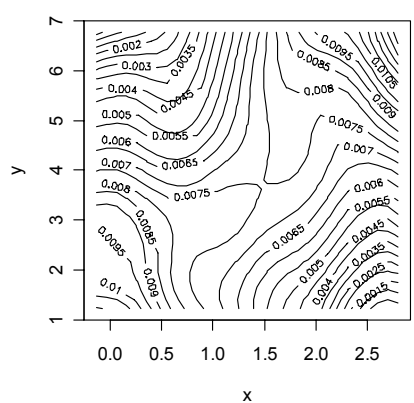

(d)

Figura 2.28 - Considerando a função de correlação cruzada contemporânea local e o modelo VAR(1) com média $(1,33 ; 4)^{\prime}, \operatorname{vec}(\Gamma(0))=(0,80 ; 0 ; 0 ; 2,86)^{\prime}$ (correlação 0,0$)$ e inovações Gaussianas, temos: (a) gráfico da função teórica, (b) gráfico das respectivas curvas de nível, (c) gráfico da função estimada e (d) gráfico das respectivas curvas de nível, estes considerando 200 séries de tamanho 500.

Tabela 2.12 - Viés, erro quadrático médio e $p$-valor do teste de normalidade de Jarque Bera, para sete pontos de grade da diagonal principal e secundária da função de correlação cruzada contemporânea local, considerando o modelo VAR(1) com média $(1,33 ; 4)^{\prime}, \operatorname{vec}(\Gamma(0))=(0,80 ; 0 ; 0 ; 2,86)^{\prime}$

(correlação 0,0) e inovações Gaussianas com 200 séries de tamanho 500.

\begin{tabular}{|l|c|c|c|c|c|c|c|}
\hline Grade X & $\mathbf{- 0 , 1 4}$ & $\mathbf{0 , 3 5}$ & $\mathbf{0 , 8 4}$ & $\mathbf{1 , 3 3}$ & $\mathbf{1 , 8 2}$ & $\mathbf{2 , 3 1}$ & $\mathbf{2 , 8 0}$ \\
\hline Grade Y & $\mathbf{1 , 2 2}$ & $\mathbf{2 , 1 5}$ & $\mathbf{3 , 0 7}$ & $\mathbf{4 , 0 0}$ & $\mathbf{4 , 9 3}$ & $\mathbf{5 , 8 5}$ & $\mathbf{6 , 7 8}$ \\
\hline Viés $^{(1)}$ & 0,011 & 0,009 & 0,008 & 0,007 & 0,008 & 0,008 & 0,013 \\
\hline Viés $^{(2)}$ & 0,000 & 0,004 & 0,007 & 0,007 & 0,005 & 0,004 & 0,000 \\
\hline EQM $^{(1)}$ & 0,004 & 0,003 & 0,003 & 0,003 & 0,003 & 0,003 & 0,004 \\
\hline EQM $^{(2)}$ & 0,003 & 0,003 & 0,003 & 0,003 & 0,003 & 0,003 & 0,003 \\
\hline -valor $^{(1)}$ & 0,117 & 0,411 & 0,269 & 0,321 & 0,502 & 0,428 & 0,357 \\
\hline -valor $^{(2)}$ & 0,074 & 0,155 & 0,274 & 0,321 & 0,337 & 0,505 & 0,026 \\
\hline
\end{tabular}

(1) diagonal principal ; ${ }^{(2)}$ diagonal secundária. 
$-0.14,1.22$

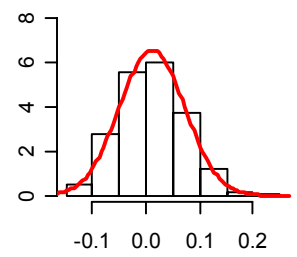

$1.82,4.93$

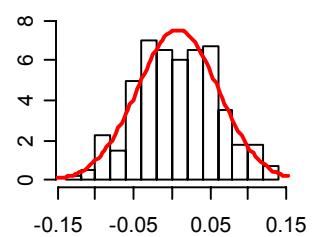

$0.35,2.15$

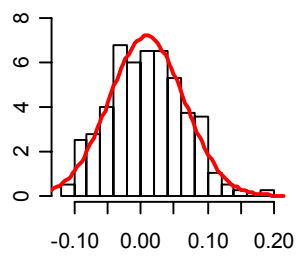

2.31, 5.85

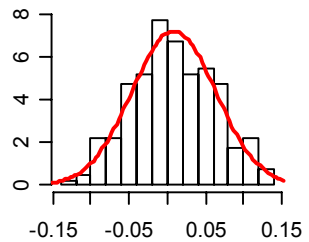

$0.84,3.07$

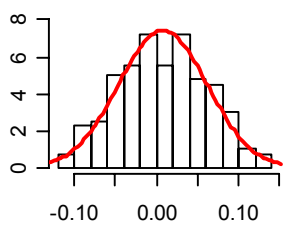

2.8, 6.78

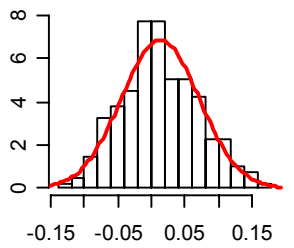

Figura 2.29 - Histogramas em pontos de grade da diagonal principal do estimador da função de correlação cruzada contemporânea local, considerando 200 séries de tamanho 500, observadas do modelo VAR(1) com média $(1,33 ; 4)^{\prime}, \operatorname{vec}(\Gamma(0))=(0,80 ; 0 ; 0 ; 2,86)^{\prime}$ (correlação 0,0$)$ e inovações Gaussianas.

$-0.14,6.78$

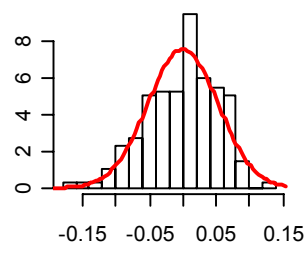

$1.82,3.07$

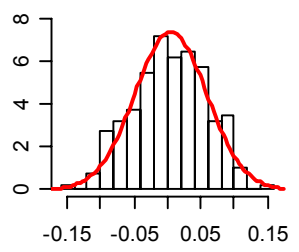

$0.35,5.85$

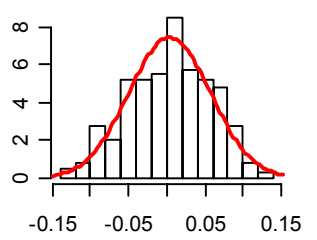

2.31, 2.15

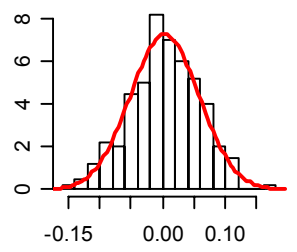

$0.84,4.93$

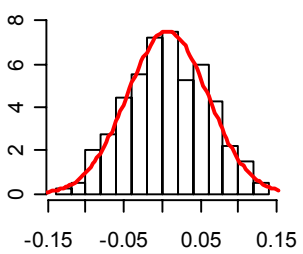

2.8, 1.22

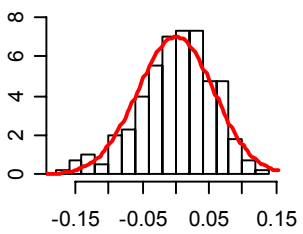

Figura 2.30 - Histogramas em pontos de grade da diagonal secundária do estimador da função de correlação cruzada contemporânea local, considerando 200 séries de tamanho 500, observadas do modelo VAR(1) com média $(1,33 ; 4)^{\prime}, \operatorname{vec}(\Gamma(0))=(0,80 ; 0 ; 0 ; 2,86)^{\prime}$ (correlação 0,0$)$ e inovações Gaussianas. 


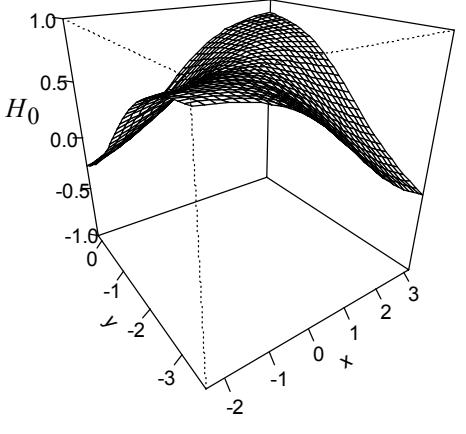

(a)

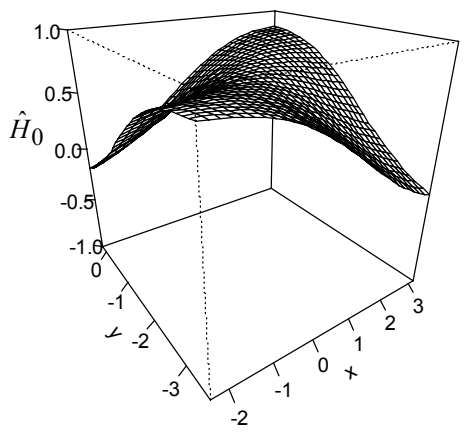

(c)

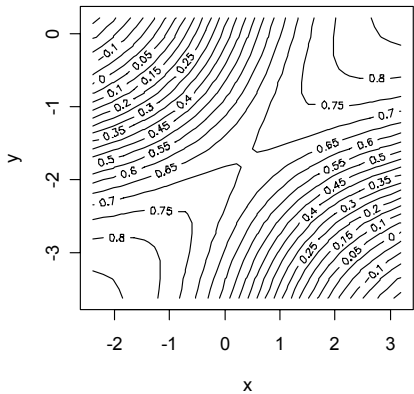

(b)

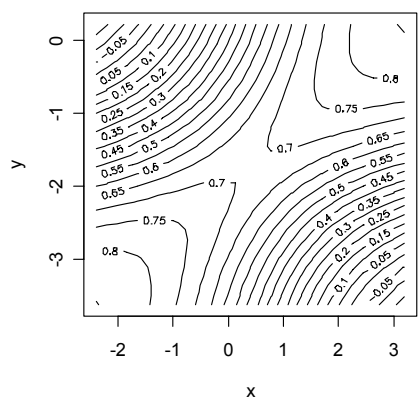

(d)

Figura 2.31 - Considerando a função de correlação cruzada contemporânea local e o modelo VMA(1) com média $(0,4 ;-1,70)^{\prime}, \operatorname{vec}(\Gamma(0))=(2,87 ; 1,39 ; 1,39 ; 1,37)^{\prime}$ (correlação 0,70$)$ e inovações Gaussianas, temos: (a) gráfico da função teórica, (b) gráfico das respectivas curvas de nível, (c) gráfico da função estimada e (d) gráfico das respectivas curvas de nível, estes considerando 200 séries de tamanho 500.

Tabela 2.13 - Viés, erro quadrático médio e $p$-valor do teste de normalidade de Jarque Bera, para sete pontos de grade da diagonal principal e secundária da função de correlação cruzada contemporânea local, considerando o modelo VMA(1) com média $(0,4 ;-1,70)^{\prime}$, vec $(\Gamma(0))=(2,87 ; 1,39 ; 1,39 ; 1,37)^{\prime}$ (correlação 0,70) e inovações Gaussianas com 200 séries de tamanho 500.

\begin{tabular}{|l|c|c|c|c|c|c|c|}
\hline Grade X & $\mathbf{- 2 , 3 9}$ & $\mathbf{- 1 , 4 6}$ & $\mathbf{- 0 , 5 3}$ & $\mathbf{0 , 4 0}$ & $\mathbf{1 , 3 3}$ & $\mathbf{2 , 2 6}$ & $\mathbf{3 , 1 9}$ \\
\hline Grade Y & $\mathbf{- 3 , 6 2}$ & $\mathbf{- 2 , 9 8}$ & $\mathbf{- 2 , 3 4}$ & $\mathbf{- 1 , 7 0}$ & $\mathbf{- 1 , 0 6}$ & $\mathbf{- 0 , 4 2}$ & $\mathbf{0 , 2 2}$ \\
\hline Viés $^{(1)}$ & $-0,019$ & $-0,015$ & $-0,008$ & $-0,004$ & $-0,009$ & $-0,015$ & $-0,017$ \\
\hline Viés $^{(2)}$ & 0,097 & 0,072 & 0,030 & $-0,004$ & 0,030 & 0,073 & 0,088 \\
\hline EQM $^{(1)}$ & 0,001 & 0,001 & 0,000 & 0,000 & 0,000 & 0,001 & 0,001 \\
\hline EQM $^{(2)}$ & 0,012 & 0,008 & 0,003 & 0,000 & 0,003 & 0,009 & 0,011 \\
\hline -valor $^{(1)}$ & 0,218 & 0,414 & 0,515 & 0,905 & 0,438 & 0,154 & 0,812 \\
\hline -valor $^{(2)}$ & 0,366 & 0,987 & 0,000 & 0,905 & 0,763 & 0,473 & 0,167 \\
\hline
\end{tabular}

(1) diagonal principal ; ${ }^{(2)}$ diagonal secundária. 

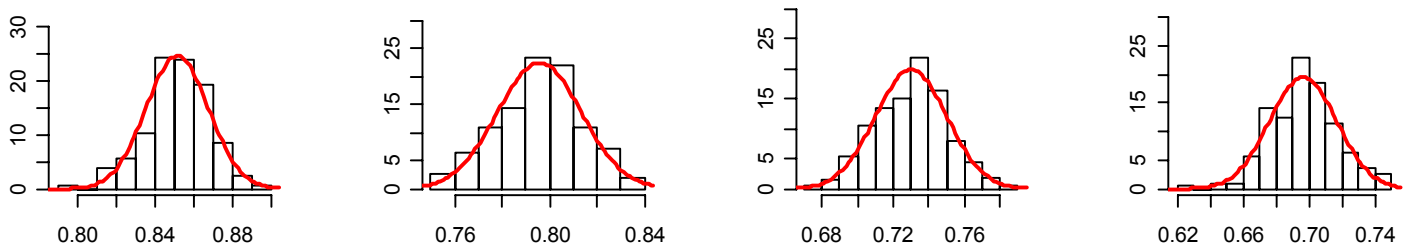

$1.33,-1.06$

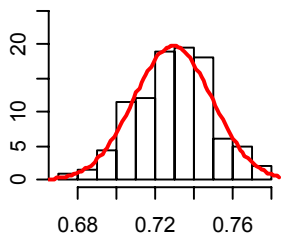

$2.26,-0.42$

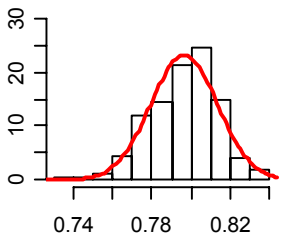

$3.19,0.22$

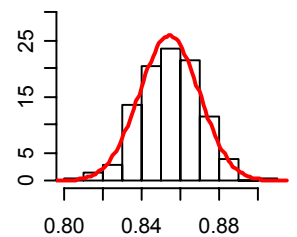

Figura 2.32 - Histogramas em pontos de grade da diagonal principal do estimador da função de correlação cruzada contemporânea local, considerando 200 séries de tamanho 500, observadas do modelo VMA(1)

com média $(0,4 ;-1,70)^{\prime}, \operatorname{vec}(\Gamma(0))=(2,87 ; 1,39 ; 1,39 ; 1,37)^{\prime}$ (correlação 0,70$)$ e inovações Gaussianas.

$-2.39,0.22$

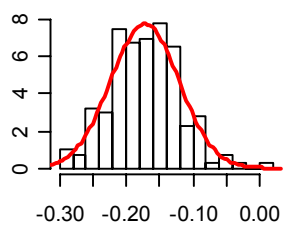

$1.33,-2.34$

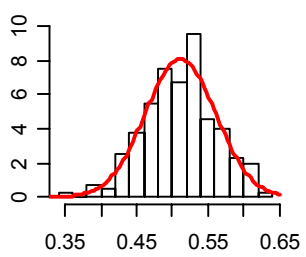

$-1.46,-0.42$

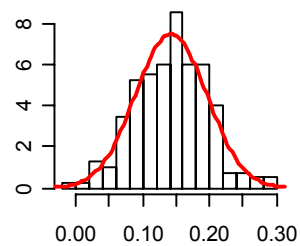

2.26, -2.98

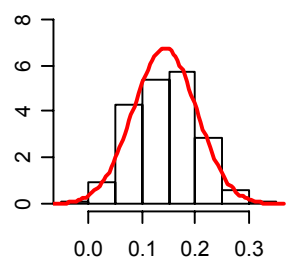

$-0.53,-1.06$

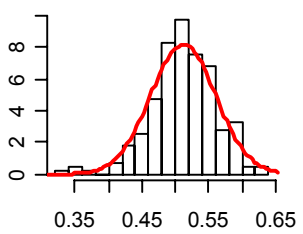

$3.19,-3.62$

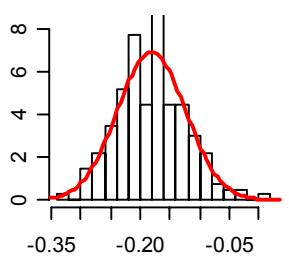

Figura 2.33 - Histogramas em pontos de grade da diagonal secundária do estimador da função de correlação cruzada contemporânea local, considerando 200 séries de tamanho 500, observadas do modelo VMA(1) com média $(0,4 ;-1,70)^{\prime}, \operatorname{vec}(\Gamma(0))=(2,87 ; 1,39 ; 1,39 ; 1,37)^{\prime}$ (correlação 0,70$)$ e inovações Gaussianas. 


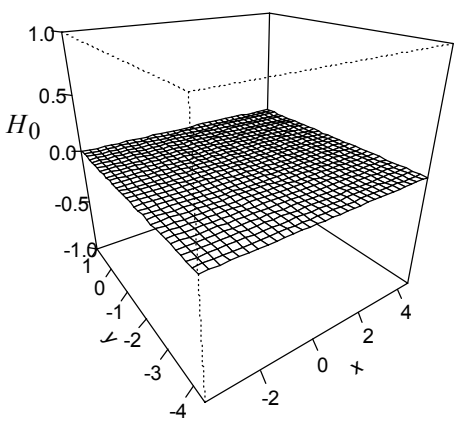

(a)

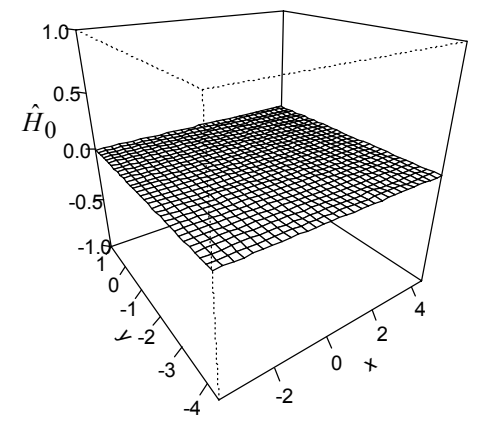

(c)

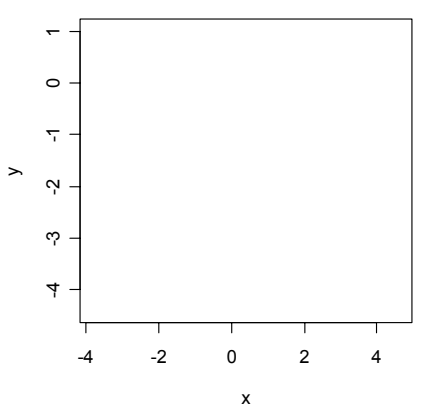

(b)

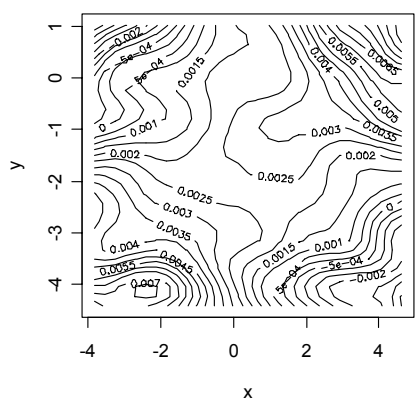

(d)

Figura 2.34 - Considerando a função de correlação cruzada contemporânea local e o modelo VMA(1) com média $(0,4 ;-1,70)^{\prime}, \operatorname{vec}(\Gamma(0))=(6,56 ; 0 ; 0 ; 2,72)^{\prime}$ (correlação 0,0$)$ e inovações Gaussianas, temos: (a) gráfico da função teórica, (b) gráfico das respectivas curvas de nível, (c) gráfico da função estimada e

(d) gráfico das respectivas curvas de nível, estes considerando 200 séries de tamanho 500.

Tabela 2.14 - Viés, erro quadrático médio e $p$-valor do teste de normalidade de Jarque Bera, para sete pontos de grade da diagonal principal e secundária da função de correlação cruzada contemporânea local, considerando o modelo VMA(1) com média $(0,4 ;-1,70)^{\prime}$, vec $(\Gamma(0))=(6,56 ; 0 ; 0 ; 2,72)^{\prime}$ (correlação 0,0) e inovações Gaussianas com 200 séries de tamanho 500.

\begin{tabular}{|l|c|c|c|c|c|c|c|}
\hline Grade X & $\mathbf{- 3 , 8 1}$ & $\mathbf{- 2 , 4 1}$ & $\mathbf{- 1 , 0 0}$ & $\mathbf{0 , 4 0}$ & $\mathbf{1 , 8 0}$ & $\mathbf{3 , 2 1}$ & $\mathbf{4 , 6 1}$ \\
\hline Grade Y & $\mathbf{- 4 , 4 1}$ & $\mathbf{- 3 , 5 1}$ & $\mathbf{- 2 , 6 0}$ & $\mathbf{- 1 , 7 0}$ & $\mathbf{- 0 , 8 0}$ & $\mathbf{0 , 1 1}$ & $\mathbf{1 , 0 1}$ \\
\hline Viés $^{(1)}$ & 0,008 & 0,005 & 0,003 & 0,003 & 0,003 & 0,005 & 0,009 \\
\hline Viés $^{(2)}$ & $-0,004$ & 0,000 & 0,002 & 0,003 & 0,002 & 0,001 & $-0,004$ \\
\hline EQM $^{(1)}$ & 0,003 & 0,003 & 0,003 & 0,003 & 0,003 & 0,003 & 0,003 \\
\hline EQM $^{(2)}$ & 0,003 & 0,003 & 0,003 & 0,003 & 0,003 & 0,003 & 0,003 \\
\hline P-valor $^{(1)}$ & 0,000 & 0,048 & 0,671 & 0,729 & 0,468 & 0,044 & 0,000 \\
\hline -valor $^{(2)}$ & 0,001 & 0,560 & 0,755 & 0,729 & 0,810 & 0,348 & 0,006 \\
\hline
\end{tabular}

(1) diagonal principal ; ${ }^{(2)}$ diagonal secundária. 
$-3.81,-4.41$

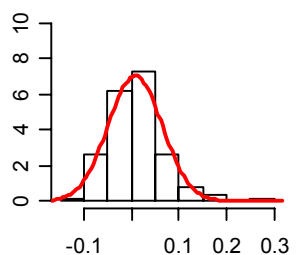

$1.8,-0.8$

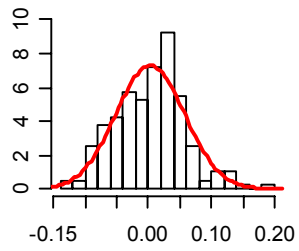

$-2.41,-3.51$

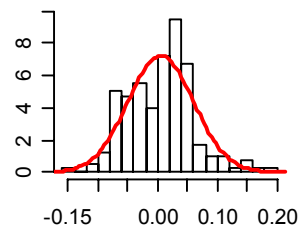

$3.21,0.11$

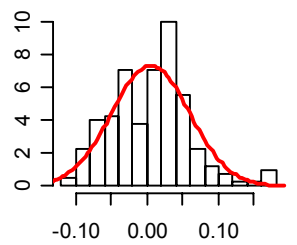

$-1,-2.6$

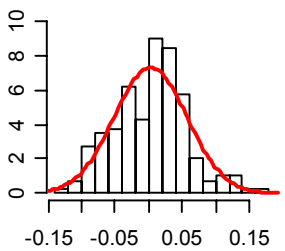

4.61, 1.01

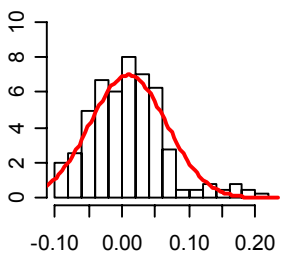

Figura 2.35 - Histogramas em pontos de grade da diagonal principal do estimador da função de correlação cruzada contemporânea local, considerando 200 séries de tamanho 500, observadas do modelo VMA(1) com média $(0,4 ;-1,70)^{\prime}, \operatorname{vec}(\Gamma(0))=(6,56 ; 0 ; 0 ; 2,72)^{\prime}$ (correlação 0,0$)$ e inovações Gaussianas.

$-3.81,1.01$

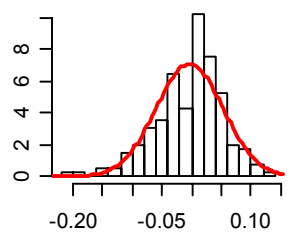

1.8, -2.6

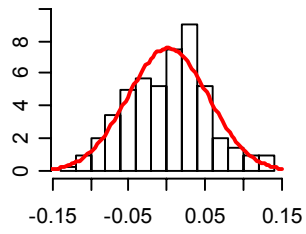

$-2.41,0.11$

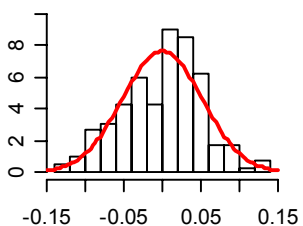

$3.21,-3.51$

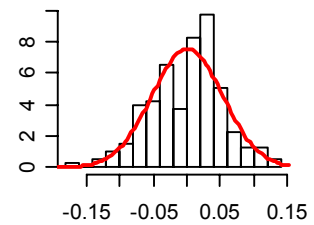

$-1,-0.8$

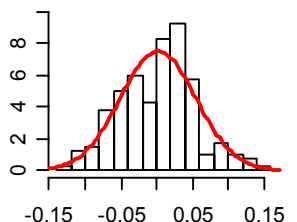

$4.61,-4.41$

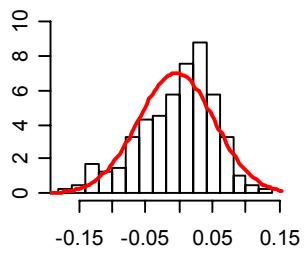

Figura 2.36 - Histogramas em pontos de grade da diagonal secundária do estimador da função de correlação cruzada contemporânea local, considerando 200 séries de tamanho 500, observadas do modelo VAR(1) com média $(0,4 ;-1,70)^{\prime}, \operatorname{vec}(\Gamma(0))=(6,56 ; 0 ; 0 ; 2,72)^{\prime}$ (correlação 0,0$)$ e inovações Gaussianas. 


\subsubsection{Aplicações a séries reais}

A seguir, avaliamos a estrutura de dependência entre os retornos diários da Petrobrás e do Ibovespa, e também do Banespa e do Ibovespa no período de 3/jan/95 a 27/dez/00, totalizando 1.498 dados cada série. Nestas simulações, utilizamos grade com $31 \times 31$ pontos, $99,4 \%$ dos dados centrais, e kernel Gaussiano com largura de faixa igual ao desvio-padrão de cada série,.

Os retornos da Petrobrás $\left(X_{t}\right)$ e do Ibovespa $\left(Y_{t}\right)$ apresentaram correlação linear de Pearson de 0,83 , rho de Spearman de 0,77 e tau de Kendall de 0,59. Na Figura 2.37, observamos que há dependência intra (f.a.c. dos retornos ao quadrado) e inter (f.a.c.c. dos retornos ao quadrado) séries. Na Figura 2.38, os três primeiros gráficos avaliam as séries dos retornos, sendo que no gráfico (a) de dispersão, observamos a indicação de forte relação linear positiva, e no gráfico (b) de perspectiva de $\hat{H}_{0}$ e no gráfico (c) de curvas de nível de $\hat{H}_{0}$, vemos que há dependência positiva tanto maior quanto maior for a proximidade dos dados em relação à diagonal principal, e alguma dependência negativa nos subquadrantes mais extremos da diagonal secundária. Os três últimos gráficos desta figura referem-se aos postos normalizados, sendo que no gráfico de dispersão em (d), vemos a existência de forte dependência positiva entre os valores baixos dos postos normalizados e também entre os valores altos destes (porém, com menor intensidade), além de uma dependência positiva mais suave entre valores intermediários. Esse comportamento é confirmado nos gráficos (e) e (f) de perspectiva e de curvas de nível da densidade cópula empírica suavizada, respectivamente.

A associação global verificada entre os retornos do Banespa $\left(X_{t}\right)$ e do Ibovespa $\left(Y_{t}\right)$ foi de 0,54 para a correlação de Pearson, de 0,48 para o rho de Spearman e 0,34 para o tau de Kendall. Na Figura 2.39, novamente observamos dependência intra e inter séries. Na Figura 2.40, podemos observar relação linear positiva entre os retornos das duas séries no gráfico (a), e dependência positiva e negativa (similar ao caso anterior, porém mais suave) nos gráficos (b) e (c). Além disso, os gráficos (d), (e) e (f) também mostram dependência positiva entre os baixos valores e entre os altos valores dos postos normalizados, além de leve dependência positiva entre valores intermediários. 


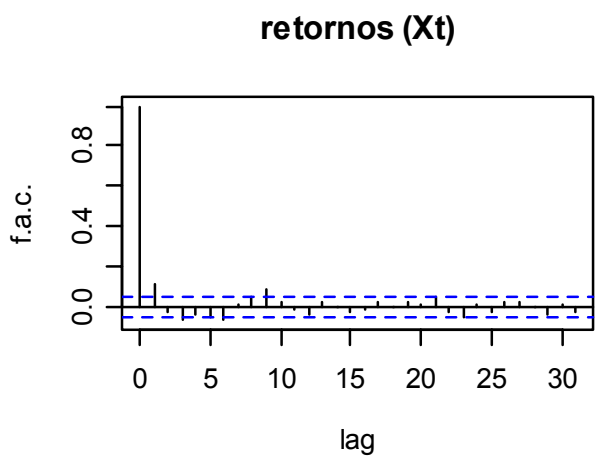

retornos $(\mathrm{Yt})$

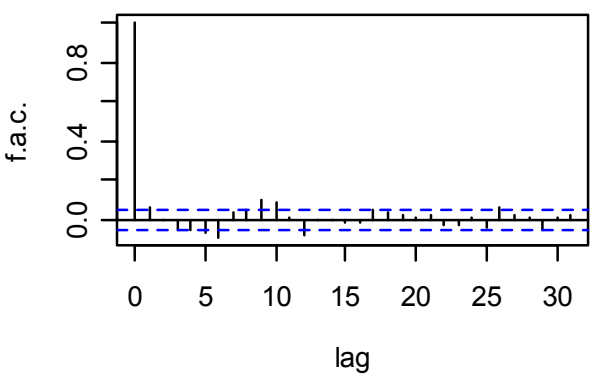

retornos (Xt e Yt)

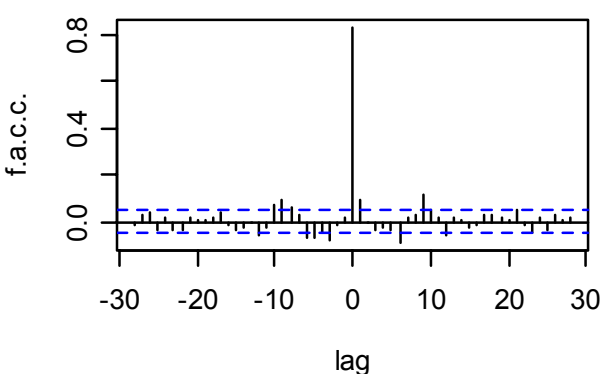

retornos^2 $\left(X^{\wedge} 2\right)$

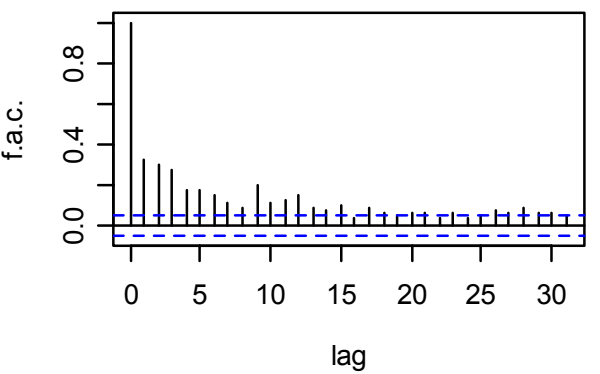

retornos^ $2\left(\mathrm{Yt}^{\wedge} 2\right)$

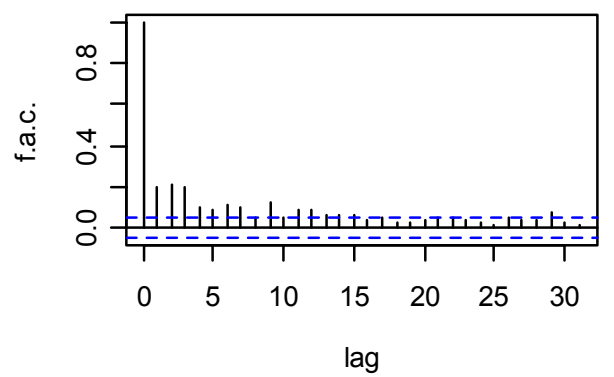

retornos^2 $\left(\mathrm{Xt}^{\wedge} 2\right.$ e $\left.\mathrm{Yt}^{\wedge} 2\right)$

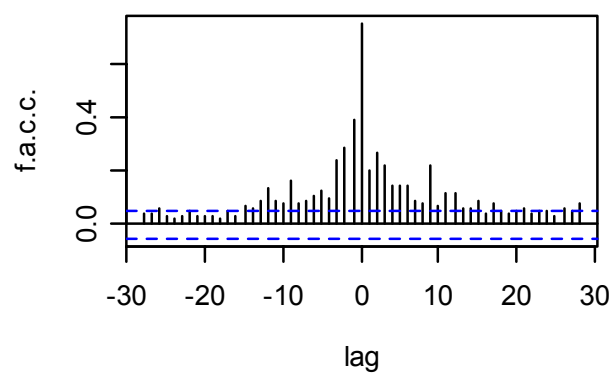

Figura 2.37 - Função de autocorrelação dos retornos e dos retornos ao quadrado da Petrobrás $\left(X_{t}\right)$ e do Ibovespa $\left(Y_{t}\right)$ (período de 3/jan/95 a 27/dez/00), e função de autocorrelação cruzada desses retornos e desses retornos ao quadrado. 


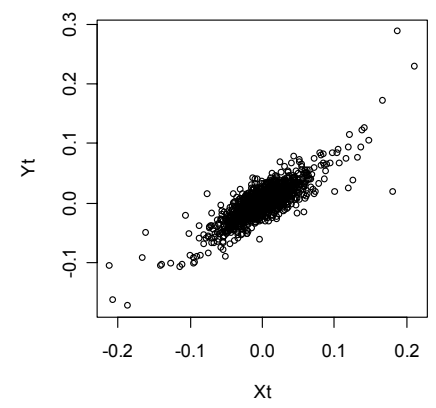

(a)

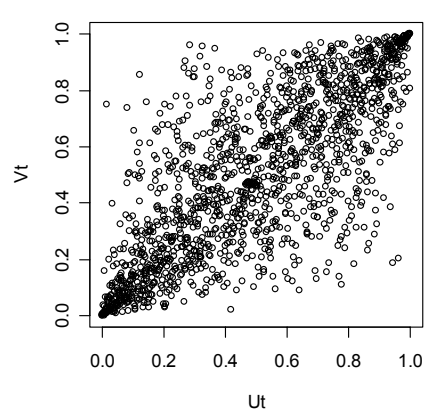

(d)

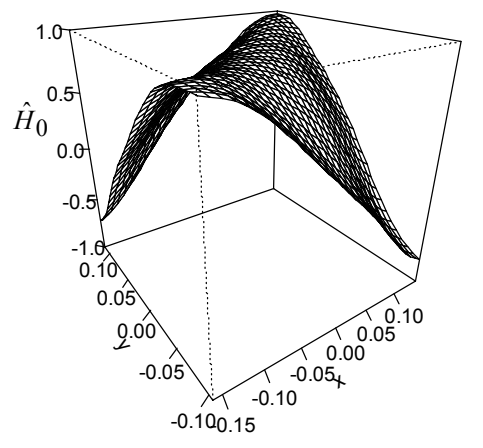

(b)

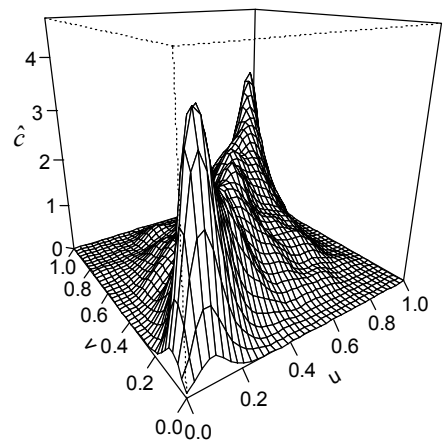

(e)

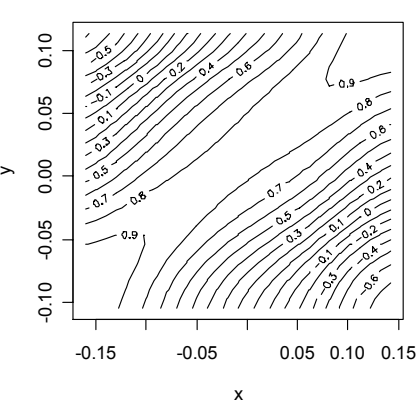

(c)

Figura 2.38 - Para os retornos diários da Petrobrás $\left(X_{t}\right)$ e do Ibovespa $\left(Y_{t}\right)$, de 3/jan/95 a 27/dez/00, temos:

(a) gráfico de dispersão, (b) função de correlação cruzada contemporânea local estimada e respectivo (c) gráfico de curvas de nível. Para os correspondentes postos normalizados, temos o (d) gráfico de dispersão, (e) densidade cópula estimada e respectivas (f) curvas de nível. 
retornos (Xt)

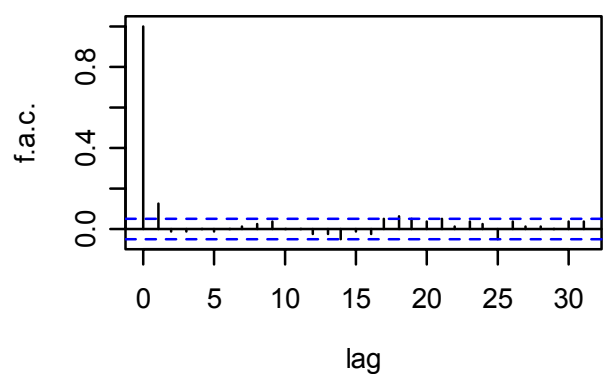

retornos (Yt)

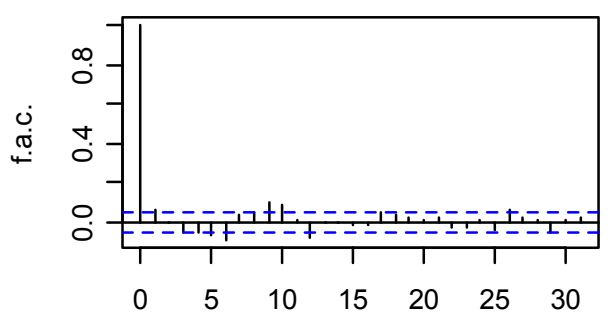

lag

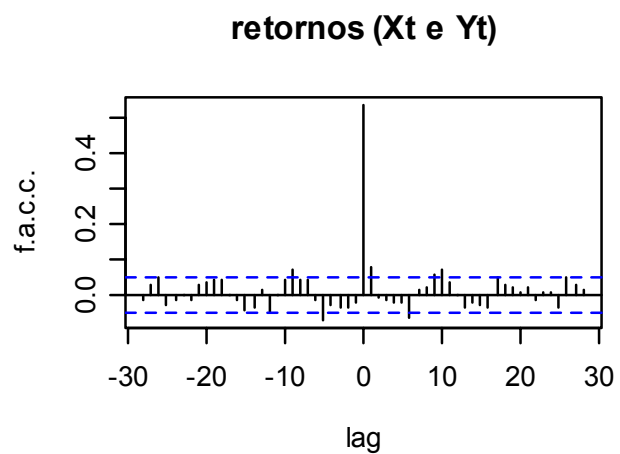

retornos ${ }^{\wedge} 2\left({X t^{\wedge}}^{\wedge}\right)$

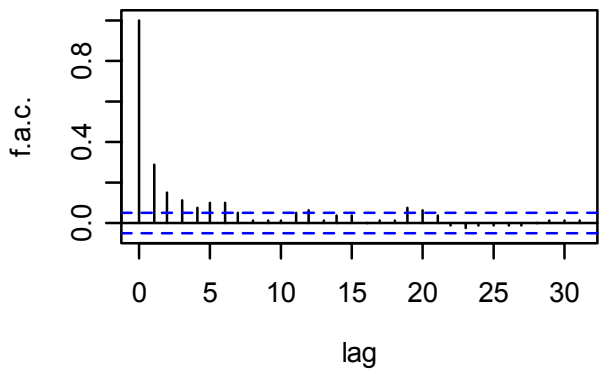

retornos^2 $\left(\mathrm{Yt}^{\wedge} 2\right)$

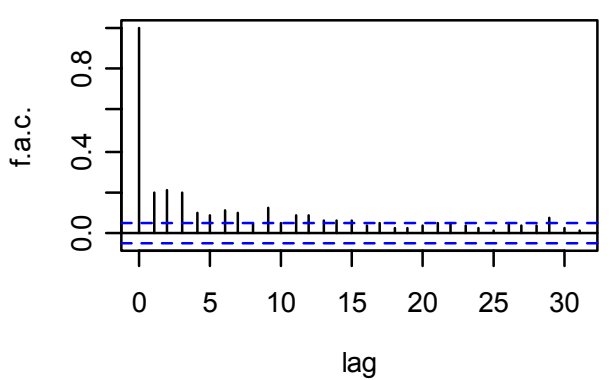

retornos^2 (Xt^2 e Yt^2)

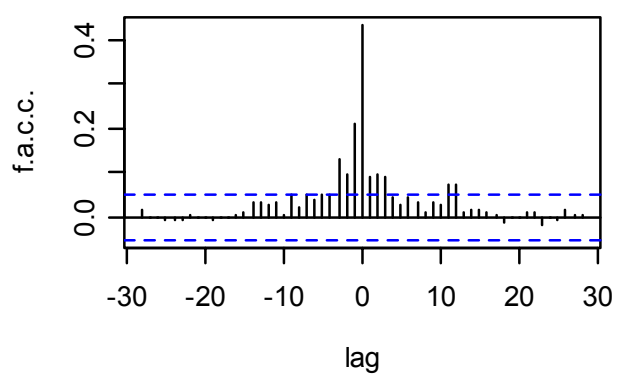

Figura 2.39 - Função de autocorrelação dos retornos e dos retornos ao quadrado da Banespa $\left(X_{t}\right)$ e do Ibovespa $\left(Y_{t}\right)$ (período de 3/jan/95 a 27/dez/00), e função de autocorrelação cruzada desses retornos e desses retornos ao quadrado. 


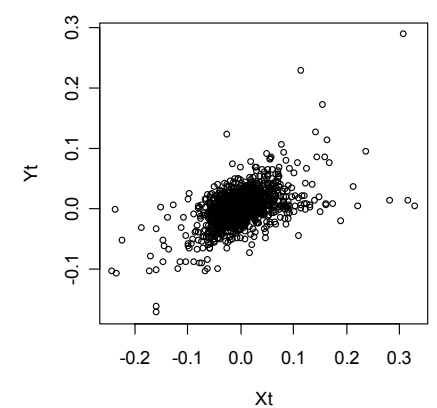

(a)

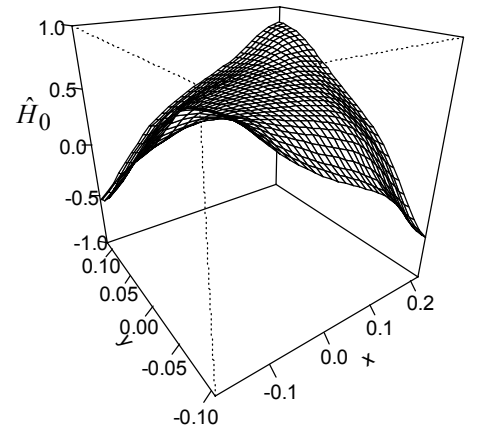

(b)

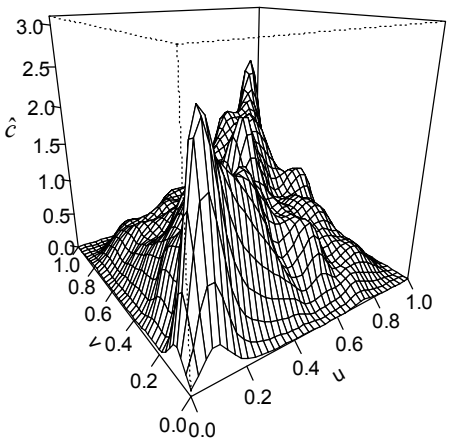

(e)

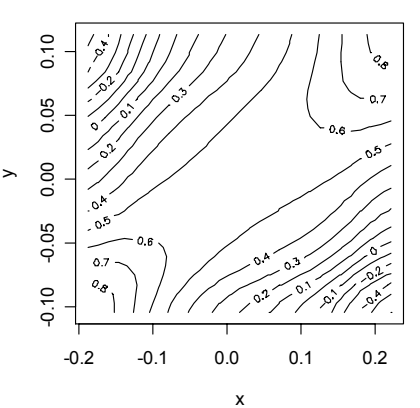

(c)

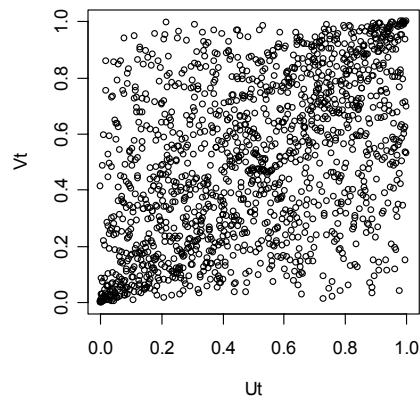

(d)

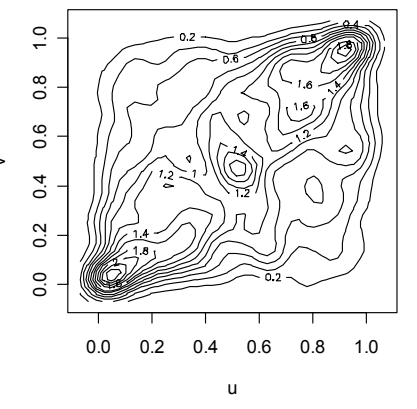

(f)

Figura 2.40 - Para os retornos diários da Banespa $\left(X_{t}\right)$ e do Ibovespa $\left(Y_{t}\right)$, de 3/jan/95 a 27/dez/00, temos: (a) gráfico de dispersão, (b) função de correlação cruzada contemporânea local estimada e respectivo (c) gráfico de curvas de nível. Para os correspondentes postos normalizados, temos o (d) gráfico de dispersão, (e) densidade cópula estimada e respectivas (f) curvas de nível. 


\section{Capítulo 3}

\section{Curva de Correlação}

\subsection{Curva de Correlação Cruzada Contemporânea}

\subsubsection{Introdução}

Considere $\left\{\left(X_{t}, Y_{t}\right), t \in Z\right\}$ um processo estacionário estrito com $X_{t}$ a valores contínuos, tal que $X_{t} \mathrm{e}$ $Y_{t}$ possuem segundos momentos finitos e valores em $S$. Sejam

$$
\begin{aligned}
& m(x)=E\left[Y_{t} / X_{t}=x\right], \\
& \sigma^{2}(x)=\operatorname{Var}\left[Y_{t} / X_{t}=x\right],
\end{aligned}
$$

respectivamente, a esperança e a variância condicional de $Y_{t}$ dado que $X_{t}=x$, com $x \in S$, as quais estão bem definidas. Como em geral a função $m(x)$ não é necessariamente linear e a variância condicional $\sigma^{2}(x)$ não é necessariamente homoscedástica, iremos considerar o modelo

$$
Y_{t}=m\left(X_{t}\right)+\sigma\left(X_{t}\right) \varepsilon_{t}, \text { com } \varepsilon_{t} \sim \operatorname{iid}(0,1) \text { e } \varepsilon_{t} \perp X_{s}, s<t
$$

sendo $m($.$) o "drift" e \sigma($.$) a "volatilidade" (Fan e Yao, 1998).$

Vejamos então como fica a expressão da curva de correlação (1.2).

Como o processo é estacionário estrito, então a distribuição de $\left(X_{t}, Y_{t}\right)$ é invariante sob translações do tempo, e $f_{Y_{t} / X_{t}}(y / x)$ (com $\left.f_{X_{t}}(x)>0\right)$ e $f_{X_{t}}(x)$ não dependem do tempo, $\forall x, y \in S$. Portanto, $\beta(x)=\partial / \partial x E\left[Y_{t} / X_{t}=x\right]=\partial / \partial x\left(\int y f_{Y_{t} / X_{t}}(y / x) d y\right), \sigma^{2}(x)=\operatorname{Var}\left[Y_{t} / X_{t}=x\right]=\int\left(y-\mu_{Y}\right)^{2} f_{Y_{t} / X_{t}}(y / x) d y \mathrm{e}$ $\gamma_{0}=\operatorname{Var}\left[X_{t}\right]$ são invariantes no tempo.

Então, a curva de correlação (1.2) pode ser escrita como

$$
\rho_{0}(x)=\frac{\beta_{0}(x) \sqrt{\gamma_{0}}}{\sqrt{\beta_{0}^{2}(x) \gamma_{0}+\sigma_{0}^{2}(x)}}, \forall x \in S \operatorname{com} f(x)>0, \forall t \in Z,
$$

em que $\beta_{0}(x)=\beta(x)$ e $\sigma_{0}^{2}(x)=\sigma^{2}(x)$ são como acima, e $f(x)=f_{X_{t}}(x)$.

Denominamos (3.2) de curva de correlação cruzada contemporânea.

\subsubsection{Propriedades}

Para o processo em estudo, a curva de correlação cruzada contemporânea (3.2) satisfaz às seguintes propriedades: 
(i) $-1 \leq \rho_{0}(x) \leq+1, \forall x \in S$;

prova:

Se $Y_{t}=g\left(X_{t}\right)$, então a variância condicional é nula (veja a propriedade (iii) a seguir), e $\rho_{0}(x)=1 \times \operatorname{sinal}\left(g^{\prime}(x)\right)$. Caso contrário, $-1<\rho_{0}(x)<+1$, que pode ser observado diretamente da expressão de $\rho_{0}(x)$ reescrita como $\rho_{0}(x)=\sqrt{\beta_{0}^{2}(x) \gamma_{0} /\left(\beta_{0}^{2}(x) \gamma_{0}+\sigma_{0}^{2}(x)\right)}$.

(ii) $\rho_{0}(x)=0, \forall x \in S$ quando $X_{t}$ e $Y_{t}$ são independentes;

prova:

Se $X_{t}$ e $Y_{t}$ são independentes, então $\beta_{0}(x)=\frac{\partial}{\partial x} E\left[Y_{t} / X_{t}=x\right]=\frac{\partial}{\partial x} E\left[Y_{t}\right]=0$, portanto, $\rho_{0}(x)=0$.

(iii) $\rho_{0}(x) \equiv \pm 1, \forall x \in S$ quando $Y_{t}$ é uma função de $X_{t}$, com $Y_{t}$ sendo não constante;

prova:

Suponha que $\quad Y_{t}=g\left(X_{t}\right)$. Então, $\quad E\left[Y_{t} / X_{t}=x\right]=E\left[g\left(X_{t}\right) / X_{t}=x\right]=g(x) \quad \mathrm{e} \quad \operatorname{Var}\left[Y_{t} / X_{t}=x\right]=$ $\operatorname{Var}\left[g\left(X_{t}\right) / X_{t}=x\right]=0$. Portanto, $\rho_{0}(x)=\frac{g^{\prime}(x) \sqrt{\gamma_{0}}}{\sqrt{g^{\prime 2}(x) \gamma_{0}}}= \pm 1$ para $g^{\prime}(x)$ e $\gamma_{0}$ não nulos, sendo $\rho_{0}(x)=1$ quando $g^{\prime}(x)>0$, e $\rho_{0}(x)=-1$ quando $g^{\prime}(x)<0$. Para $g^{\prime}(x)=0$, iremos considerar $0 / 0=1$.

(iv) $\rho_{0}(x)= \pm 1$ para quase todo $x$, implica que $Y_{t}$ é uma função de $x$;

prova:

Se $\rho_{0}(x)= \pm 1$ para quase todo $x$, então $\sigma_{0}^{2}(x)=0$, que implica em $Y_{t}=g(x)$ para quase todo $x$, para alguma função $g$.

(v) $U_{t}=a+b X_{t}$ e $V_{t}=c+d Y_{t}$ com $b d \neq 0$, então

$$
\rho_{U_{t} V_{t}}(u)=\operatorname{sinal}(b d) \rho_{X_{t} Y_{t}}(x), \forall u \in S \text { em que } x=\frac{u-a}{b} .
$$

Notação: $\rho_{X_{t} Y_{t}}(x)$ indica $\rho_{0}(x)$ para $\left(X_{t}, Y_{t}\right)$ com o condicionamento $Y_{t} / X_{t}=x$;

prova: Imediata.

(vi) $\rho_{X_{t} Y_{t}}(x) \neq \rho_{Y_{t} X_{t}}(x), x \in S, t \in Z$, de modo geral;

prova: Imediata.

(vii) $\rho_{0}(x)=\rho_{X Y}, \forall x \in S$ no caso normal bivariado.

prova:

Se $\left(X_{t}, Y_{t}\right) \sim N_{2}$, então $Y_{t} / X_{t}=x \sim N\left(\mu_{Y}+\gamma_{X Y}\left(x-\mu_{X}\right) / \gamma_{0}, \sigma_{Y}^{2}\left(1-\rho_{X Y}^{2}\right)\right)$ e $\rho_{X Y}=\gamma_{X Y} / \sqrt{\gamma_{0}} \sigma_{Y}$.

Portanto, $\beta_{0}(x)=\frac{\partial}{\partial x} E\left[Y_{t} / X_{t}=x\right]=\frac{\gamma_{X Y}}{\gamma_{0}}=\rho_{X Y} \frac{\sigma_{Y}}{\sqrt{\gamma_{0}}}$. Então, 


$$
\rho_{0}(x)=\frac{\beta_{0}(x) \sqrt{\gamma_{0}}}{\sqrt{\beta_{0}^{2}(x) \gamma_{0}+\sigma_{0}^{2}(x)}}=\frac{\rho_{X Y} \sigma_{Y} \sqrt{\gamma_{0}} / \sqrt{\gamma_{0}}}{\sqrt{\rho_{X Y} \sigma_{Y}^{2} \gamma_{0} / \gamma_{0}+\sigma_{Y}^{2}\left(1-\rho_{X Y}^{2}\right)}}=\rho_{X Y} .
$$

\subsubsection{Estimador}

Modelos não lineares para séries temporais podem ser estimados por meio de uma abordagem não paramétrica. $\mathrm{O}$ ajuste polinomial local é uma técnica de regressão não paramétrica conveniente para estimar a $k$-ésima derivada $m^{(k)}(x)$ da função de regressão $m(x)=E\left[Y_{t} / X_{t}=x\right]$. A forma da função $m($.) não é conhecida, porém, precisamos supor que ela tenha um certo grau de suavidade, ou seja, $m(x)$ possui um certo número de derivadas no ponto $x_{0}$. Além disso, considerando o enfoque local, um ponto distante de $x_{0}$ fornece pouca informação sobre $m(x)$. Então, supondo que $m(x)$ tenha $p+1$ derivadas no ponto $x_{0}$, a expansão de Taylor para $x$ numa vizinhança local de $x_{0}$, é dada por

$$
m(x)=m^{(0)}\left(x_{0}\right)+m^{(1)}\left(x_{0}\right)\left(x-x_{0}\right)+m^{(2)}\left(x_{0}\right) \frac{\left(x-x_{0}\right)^{2}}{2 !}+\ldots+m^{(p)}\left(x_{0}\right) \frac{\left(x-x_{0}\right)^{p}}{p !}+O\left\{\left(x-x_{0}\right)^{p+1}\right\} .
$$

Em termos de modelagem estatística, localmente ao redor de $x_{0}, m(x)$ é modelada como

$$
m(x) \approx \sum_{k=0}^{p} \alpha_{k}\left(x-x_{0}\right)^{k}
$$

com $\alpha_{k}=m^{(k)}\left(x_{0}\right) / k$ !. Então, observados $\left\{\left(X_{t}, Y_{t}\right), t=1, \ldots, T\right\}$, este modelo é ajustado utilizando-se o método de mínimos quadrados ponderados, minimizando-se

$$
\sum_{t=1}^{T}\left(Y_{t}-\sum_{k=0}^{p} \alpha_{k}\left(X_{t}-x_{0}\right)^{k}\right)^{2} K_{h_{T}}\left(X_{t}-x_{0}\right)
$$

em que $K$ é uma função kernel e $h_{T}$ é a largura de faixa que controla o tamanho da vizinhança local. A solução deste método é dada por $\hat{\alpha}_{k}=\hat{m}^{(k)}\left(x_{0}\right) / k$ !, ou seja, $\hat{m}^{(k)}\left(x_{0}\right)=\hat{\alpha}_{k} \times k !, k=0, \ldots, p$.

Utilizando a notação matricial, temos a seguinte representação:

$$
\min _{\boldsymbol{\alpha}}(\mathbf{y}-\mathbf{X} \boldsymbol{\alpha})^{\prime} \mathbf{W}(\mathbf{y}-\mathbf{X} \boldsymbol{\alpha})
$$

em que

$$
\begin{aligned}
& \mathbf{y}=\left[\begin{array}{c}
Y_{1} \\
\vdots \\
Y_{T}
\end{array}\right], \quad \mathbf{X}=\left[\begin{array}{cccc}
1 & \left(X_{1}-x_{0}\right) & \ldots & \left(X_{1}-x_{0}\right)^{p} \\
\vdots & \vdots & & \vdots \\
1 & \left(X_{T}-x_{0}\right) & \ldots & \left(X_{T}-x_{0}\right)^{p}
\end{array}\right], \quad \boldsymbol{\alpha}=\left(\alpha_{0}, \ldots, \alpha_{p}\right)^{\prime}, \\
& \mathbf{W}=\operatorname{diag}\left\{K_{h_{T}}\left(X_{t}-x_{0}\right)\right\}: \text { matriz diagonal }(T \times T) \text { de pesos, }
\end{aligned}
$$

e cujo vetor solução é dado por $\hat{\boldsymbol{\alpha}}=\left(\mathbf{X}^{\prime} \mathbf{W} \mathbf{X}\right)^{-1} \mathbf{X}^{\prime} \mathbf{W} \mathbf{y}, \hat{\boldsymbol{\alpha}}=\left(\hat{\alpha}_{0}, \ldots, \hat{\alpha}_{p}\right)^{\prime}$.

Vejamos como estimar a derivada (de ordem $k=1$ ) da esperança condicional, $\beta\left(x_{0}\right)$, com $x_{0} \in G$, sendo $G$ o conjunto dos pontos de uma grade (univariada). Considerando um kernel $K_{1}$ e uma largura de faixa $h_{1}=\hat{h}_{T}\left(X_{1}, \ldots, X_{T} ; Y_{1}, \ldots, Y_{T}\right)$, ajustamos um polinômio de grau $p=2(p=k+1$ - mais detalhes no final desta seção), ou seja, minimizamos 


$$
\sum_{t=1}^{T}\left(Y_{t}-m^{(0)}\left(x_{0}\right)-m^{(1)}\left(x_{0}\right)\left(X_{t}-x_{0}\right)-m^{(2)}\left(x_{0}\right) \frac{\left(X_{t}-x_{0}\right)^{2}}{2}\right)^{2} \frac{1}{h_{1}} K_{1}\left(\frac{X_{t}-x_{0}}{h_{1}}\right),
$$

e, então, $\hat{\beta}\left(x_{0}\right)=\hat{m}^{(1)}\left(x_{0}\right)$.

Agora, vejamos como estimar a variância condicional $\sigma^{2}\left(x_{0}\right)$. Sabemos que $\sigma^{2}(x)$ pode ser escrita como $\operatorname{Var}\left[Y_{t} / X_{t}=x\right]=E\left[Y_{t}^{2} / X_{t}=x\right]-E^{2}\left[Y_{t} / X_{t}=x\right]$, que naturalmente motiva 0 estimador $\hat{E}\left[Y_{t}^{2} / X_{t}=x_{0}\right]-\left(\hat{E}\left[Y_{t} / X_{t}=x_{0}\right]\right)^{2}$. Mas, segundo Fan e Yao (1998) este estimador nem sempre é não negativo, principalmente quando diferentes larguras de faixa são utilizadas para estimar cada uma das componentes. Então, tanto Bjerve e Doksum (1993) quanto Fan e Yao (2005), dentre outros, utilizam um estimador com base em resíduos ao quadrado, ou seja, $\hat{\sigma}^{2}\left(x_{0}\right)=\hat{E}\left[\hat{r}_{t}^{2} / X_{t}=x_{0}\right]=\hat{E}\left[\left\{Y_{t}-\hat{m}\left(X_{t}\right)\right\}^{2} / X_{t}=x_{0}\right]$ (veja detalhes a seguir). Pode-se mostrar que este estimador é completamente adaptativo em regressão, isto é, sem conhecer $m\left(\right.$.), podemos estimar assintoticamente a variância condicional $\sigma^{2}($.) tão bem quanto se $m($.) fosse conhecida. De fato, pode-se provar que $E\left[r_{t}^{2} / X_{t}\right] \approx \sigma^{2}\left(X_{t}\right)$ (veja Fan e Yao, 2005 - página 375).

Então, para estimarmos $\sigma^{2}\left(x_{0}\right)=E\left[\left\{Y_{t}-m\left(X_{t}\right)\right\}^{2} / X_{t}=x_{0}\right]$, inicialmente calculamos $\hat{m}\left(X_{t}\right), t=1, \ldots, T$, usando a largura de faixa $h_{1}$ dada anteriormente, e então calculamos $\hat{r}_{t}^{2}=Y_{t}-\hat{m}\left(X_{t}\right), t=1, \ldots, T$. Por fim, obtemos $\hat{\sigma}^{2}\left(x_{0}\right)$ através da minimização do seguinte polinômio linear, o qual utiliza um kernel $K_{2}$ e uma nova largura de faixa $h_{2}=\hat{h}_{T}\left(X_{1}, \ldots, X_{T} ; \hat{r}_{1}^{2}, \ldots, \hat{r}_{T}^{2}\right)$ :

$$
\sum_{t=1}^{T}\left\{\hat{r}_{t}^{2}-m^{(0)}\left(x_{0}\right)-m^{(1)}\left(x_{0}\right)\left(X_{t}-x_{0}\right)\right\}^{2} \frac{1}{h_{2}} K_{2}\left(\frac{X_{t}-x_{0}}{h_{2}}\right) .
$$

Então, $\hat{\sigma}^{2}\left(x_{0}\right)=\hat{m}^{(0)}\left(x_{0}\right)$.

Neste algorítmo, o método de seleção da largura de faixa é flexível.

Portanto, observados $\left\{\left(X_{t}, Y_{t}\right), t=1, \ldots, T\right\}$, a estimação da curva de correlação cruzada contemporânea (3.2) pode ser feita através do seguinte estimador:

$$
\hat{\rho}_{0}(x)=\frac{\hat{\beta}_{0}(x) \sqrt{c_{0}}}{\sqrt{\hat{\beta}_{0}^{2}(x) c_{0}+\hat{\sigma}_{0}^{2}(x)}}
$$

em que

$$
c_{0}=\hat{\gamma}_{0}=\frac{1}{T} \sum_{t=1}^{T}\left(X_{t}-\bar{X}\right)^{2},
$$

$\hat{\beta}_{0}(x)=\hat{m}^{(1)}(x)$ utilizando um polinômio quadrático local com kernel $K_{1}$, largura de faixa $h_{1}$ e o conjunto de dados $\left\{\left(X_{t}, Y_{t}\right), t=1, \ldots, T\right\}$,

$\hat{\sigma}_{0}^{2}(x)=\hat{m}^{(0)}(x)$ utilizando um polinômio linear local, um kernel $K_{2}$, uma largura de faixa $h_{2}$ e o conjunto de dados $\left\{\left(X_{t}, \hat{r}_{t}^{2}\right), t=1, \ldots, T\right\}$, sendo $\hat{r}_{t}=Y_{t}-\hat{m}\left(X_{t}\right)$ com $\hat{m}\left(X_{t}\right)$ sendo previsto após o ajuste de um polinômio linear local nos pontos de grade $x \in G$, que utiliza o kernel $K_{1}$, a largura de faixa $h_{l}$ e o conjunto de dados $\left\{\left(X_{t}, Y_{t}\right), t=1, \ldots, T\right\}$. 
Vejamos algumas considerações referentes à implementação do estimador polinomial local.

Precisamos escolher a largura de faixa $h_{T}$, o kernel $K$ e a ordem $p$ do polinômio, sendo que estes parâmetros são naturalmente relacionados entre si.

A largura de faixa $h_{T}$ controla a complexidade do ajuste polinomial local (quando $h_{T}=\infty$, este ajuste torna-se um ajuste polinomial global). Para seqüências estacionárias de dados sob certas condições mixing, a suavização no domínio do espaço apresenta desempenho similar à regressão não paramétrica para dados independentes. Aqui, a largura de faixa pode ser escolhida através dos seguintes critérios: validação cruzada, método de substituição pré-assintótico de Fan e Gijbels (1995) e método de substituição assintótico de Ruppert et al. (1995).

Quanto ao kernel, é mostrado por Fan et al. (1996) que, para todas as escolhas de $p$ e $k$, o kernel ótimo é o de Epanechnikov e, como mostrado em Fan e Yao (2005, §5.5), outros kernels têm aproximadamente a mesma eficiência para uso prático de $p$ e $k$. Portanto, esta escolha não é crítica.

Finalmente, a ordem do polinômio $p=k+1$ é recomendada por Fan e Gijbels (1996), com base em considerações teóricas e práticas. Portanto, se o objetivo principal é estimar a função de regressão $m(x)$, optamos pelo ajuste linear local, se o objetivo é estimar $m^{(1)}(x)$, o ajuste quadrático local é o recomendado, e assim sucessivamente.

\subsubsection{Consistência do estimador}

Sejam as seguintes condições de regularidade:

(C1) $X_{t}=\mu+\sum_{j=-\infty}^{+\infty} \alpha_{j} e_{t-j}$, em que $e_{t} \sim i i d\left(0, \sigma^{2}\right), E\left[e_{t}^{4}\right]=\eta \sigma^{4}, \sum_{j=-\infty}^{+\infty}\left|\alpha_{j}\right|<\infty$ e $\sum_{k=-\infty}^{+\infty}\left|\gamma_{k}\right|<\infty$;

(C2) O kernel $K_{1}$ é uma função limitada e com suporte limitado;

(C3) $h_{1}=O\left\{T^{-1 /(2 p+3)}\right\}$, sendo $p$ a ordem do polinômio;

(C4) A densidade condicional $f_{X_{t}, X_{t+k} / Y_{t}, Y_{t+k}}\left(x_{1}, x_{2} / y_{1}, y_{2}\right) \leq A_{1}<\infty, \forall t \in Z, \forall k \geq 1$;

(C5) Para processos $\rho$-mixing é suposto que $\sum_{k} \rho_{k}<\infty$ e $E\left[Y_{t}^{2}\right]<\infty, \forall t \in Z$. Para processos $\alpha$-mixing é suposto que $\sum_{k} k^{a}[\alpha(k)]^{1-2 / \lambda}<\infty, E\left|Y_{t}\right|^{\lambda}<\infty$ e $f_{X_{t} / Y_{t}}(x / y) \leq A_{2}<\infty$, para algum $\lambda>2$ e $a>1-2 / \lambda, \forall t \in Z$;

(C6) Para processos $\rho$-mixing e $\alpha$-mixing é suposto, respectivamente, que $\left(T / h_{1}\right)^{1 / 2} \rho\left(s_{T}\right) \rightarrow 0$ e $\left(T / h_{1}\right)^{1 / 2} \alpha\left(s_{T}\right) \rightarrow 0$, conforme $T \rightarrow \infty$. Aqui, $s_{T}$ é uma seqüência de inteiros positivos tal que $s_{T} \rightarrow \infty$ e $s_{T}=o\left\{\left(T h_{1}\right)^{1 / 2}\right\}$, conforme $T \rightarrow \infty$;

(C7) $\sigma_{0}^{2}($.) e $f($.$) são contínuas no ponto x$, e $f(x)>0$;

(C8) Para um dado ponto $x, f(x)>0, \sigma_{0}^{2}(x)>0$ e as funções $E\left[Y_{t}^{k} / X_{t}=x\right], \forall t \in Z$, são contínuas em $x$ para $k=3,4$. Além disso, $\ddot{m}(z) \equiv \partial^{2} m(z) / \partial z^{2}$ e $\ddot{\sigma}_{0}^{2}(z) \equiv \partial^{2}\left\{\sigma_{0}^{2}(z)\right\} / \partial z^{2}$ são uniformemente contínuas num conjunto aberto contendo o ponto $x$; 
(C9) $E\left[Y_{t}^{4(1+\delta)}\right]<\infty, \forall t \in Z$, em que $\delta \in[0,1)$ é uma constante ;

(C10) As funções kernels $K_{1}$ e $K_{2}$ são funções densidades simétricas, cada uma com suporte limitado. Mais ainda, $\quad\left|K_{1}\left(x_{1}\right)-K_{1}\left(x_{2}\right)\right| \leq c\left|x_{1}-x_{2}\right|, \quad\left|K_{2}\left(x_{1}\right)-K_{2}\left(x_{2}\right)\right| \leq c\left|x_{1}-x_{2}\right|, \quad$ e $\quad$ também $\left|f\left(x_{1}\right)-f\left(x_{2}\right)\right| \leq c\left|x_{1}-x_{2}\right|$ para $x_{1}, x_{2} \in R$

(C11) O processo $\left(X_{t}, Y_{t}\right)$ é absolutamente regular, isto é

$$
\beta(k) \equiv \sup _{i \geq 1} E\left\{\sup _{A \in F_{i+k}^{\infty}}\left|P\left[A / \mathcal{F}_{1}^{i}\right]-P[A]\right|\right\} \rightarrow 0, \text { conforme } k \rightarrow \infty,
$$

em que $\mathcal{F}_{u}^{v}$ é a $\sigma$-álgebra gerada por $\left\{\left(X_{t}, Y_{t}\right): t=u, \ldots, v\right\} \quad(v \geq u)$. Mais ainda, para o mesmo $\delta$ da condição (C9), $\sum_{k=1}^{\infty} k^{2} \beta^{\delta /(1+\delta)}(k)<\infty$. Convenção: $0^{0}=0$;

(C12) Conforme $T \rightarrow \infty, h_{i} \rightarrow 0$ e $\liminf T h_{i}^{4}>0, i=1,2$.

Teorema 3.1. Considere um processo $\left\{\left(X_{t}, Y_{t}\right), t \in Z\right\}$ estritamente estacionário, com $X_{t}$ e $Y_{t}$ possuindo segundos momentos finitos. Satisfeitas as condições (C1) a (C12), temos que

$$
\hat{\rho}_{0}(x) \underset{\mathrm{T} \rightarrow \infty}{\stackrel{P}{\longrightarrow}} \rho_{0}(x) \text {, para cada } x \in S \text { fixado. }
$$

prova:

Por (C1) e pelo Teorema 6.2.2 de Fuller (1976), $c_{\tau}$ é consistente para $\gamma_{\tau}$, portanto, $c_{0} \underset{T \rightarrow \infty}{\stackrel{P}{\longrightarrow} \gamma_{0}}$.

Agora, vamos verificar a convergência de $\hat{\beta}_{0}(x)$. Pelo Teorema 6.3 de Fan e Yao (2005), temos que sob as condições de regularidade (C2) a (C7), para $T \rightarrow \infty$,

$$
\sqrt{T h_{1}^{2 v+1}}\left[\hat{m}^{(v)}(x)-m^{(v)}(x)-\int u^{p+1} K_{v}^{*}(u) d u \frac{v ! m^{(p+1)}(x)}{(p+1) !} h_{1}^{p+1-v}\right] \underset{D}{\longrightarrow} N\left(0, \frac{(v !)^{2} \sigma_{0}^{2}(x) \int K_{v}^{* 2}(u) d u}{f(x)}\right),
$$

com $K_{v}^{*}(u)=\mathbf{e}_{v+1}^{T} \mathbf{S}^{-1}\left(1, u, \ldots, u^{p}\right)^{T} K(u)$, sendo $\mathbf{e}_{v+1}$ o vetor unitário com 1 na $(v+1)$-ésima posição e $\mathbf{S}$ uma matriz $(p+1) \times(p+1)$ cujo elemento $(i, j)$ é $\mu_{i+j-2}=\int_{-\infty}^{+\infty} u^{i+j-2} K(u) d u$. E quando $T \rightarrow \infty, h_{1}^{p+1-v} \rightarrow 0$.

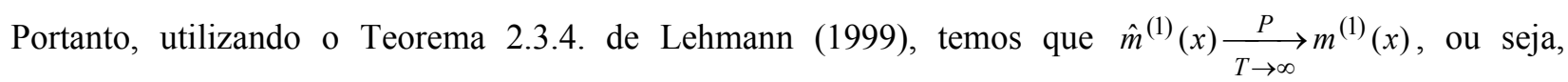
$\hat{\beta}_{0}(x) \underset{T \rightarrow \infty}{\stackrel{P}{\longrightarrow}} \beta_{0}(x)$.

Satisfeitas as condições de regularidade (C8) a (C12), o Teorema 8.5 de Fan e Yao (2005) é válido, ou seja, para $T \rightarrow \infty$,

$$
\sqrt{T h_{2}}\left(\hat{\sigma}_{0}^{2}(x)-\sigma_{0}^{2}(x)-\theta_{T}\right) \stackrel{D}{\longrightarrow} N\left(0, \frac{\sigma_{0}^{4}(x) \lambda^{2}(x)}{f(x)} \int K_{2}^{2}(u) d u\right)
$$


$\operatorname{com} \theta_{T}=\left(h_{2}^{2} / 2\right) \ddot{\sigma}_{0}^{2}(x) \int u^{2} K_{2}(u) d u+o\left(h_{1}^{2}+h_{2}^{2}\right), \lambda^{2}(x)=E\left[\left(e_{t}^{2}-1\right)^{2} / X_{t}=x\right]$ e $e_{t}=\left(Y_{t}-m\left(X_{t}\right)\right) / \sigma_{0}\left(X_{t}\right)$. Como para $T \rightarrow \infty, \quad h_{2}^{2} \rightarrow 0$, novamente pelo Teorema 2.3.4. de Lehmann (1999), temos que $\hat{\sigma}_{0}^{2}(x) \underset{T \rightarrow \infty}{\stackrel{P}{\longrightarrow}} \sigma_{0}^{2}(x)$.

Considere $\hat{\boldsymbol{\theta}}=\left(\hat{\beta}_{0}(x), \hat{\sigma}_{0}^{2}(x), c_{0}\right)^{\prime}=\left(\hat{\theta}_{1}, \hat{\theta}_{2}, \hat{\theta}_{3}\right)^{\prime} \quad$ e $\quad \boldsymbol{\theta}=\left(\beta_{0}(x), \sigma_{0}^{2}(x), \gamma_{0}\right)^{\prime}=\left(\theta_{1}, \theta_{2}, \theta_{3}\right)^{\prime}$. Verificamos, para todo $x \in S$ com $f(x)>0$, que $\hat{\theta}_{j} \stackrel{P}{\longrightarrow} \theta_{j}, j=1,2,3$, com $h_{i} \rightarrow 0$ e $\liminf T h_{i}^{4}>0, i=1,2$, conforme $T \rightarrow \infty$. Portanto, usando o Lema 5.1.3 e o Teorema 5.1.4 de Fuller (1976), temos que $\hat{\rho}_{0}(x) \stackrel{P}{\longrightarrow} \rho_{0}(x)$, conforme $T \rightarrow \infty$, para cada $x \in S$ fixado.

Fan e Yao (2005, página 395) observam que as condições dos suportes de $K_{1}($.$) e K_{2}($.$) serem$ limitados, facilita as provas dos teoremas. Na prática, o kernel Gaussiano pode ser utilizado. Além disso, a suposição sobre a taxa de convergência de $\beta(k)$ é para conveniência técnica, sendo que o resultado também pode ser estabelecido para outros tipos de coeficientes mixing. Finalmente, as taxas de convergência de $h_{1} \mathrm{e}$ $h_{2}$ da condição (C12) não são as menores possíveis.

\subsubsection{Simulações}

A curva de correlação cruzada contemporânea foi estudada utilizando-se os modelos bivariados VAR(1) e VMA(1) estacionários. Para cada modelo, consideramos 200 réplicas de Monte Carlo com diferentes tamanhos de séries (200, 500 e 1.000), grade com 199 pontos, 90\% dos dados centrais, kernel Gaussiano e largura de faixa ótima de Ruppert et al. (1995).

O modelo VAR(1) estacionário considerado foi

$$
\mathbf{Z}_{t}=\boldsymbol{\Phi}_{0}+\boldsymbol{\Phi}_{1} \mathbf{Z}_{t}+\boldsymbol{\varepsilon}_{t},
$$

em que $\quad \mathbf{Z}_{t}=\left(X_{t}, Y_{t}\right)^{\prime}, \quad \boldsymbol{\Phi}_{0}=(1,1)^{\prime}, \quad \operatorname{vec}\left(\boldsymbol{\Phi}_{1}\right)=(0,25 ; 0,2 ; 0,2 ; 0,75)^{\prime} \quad$ e $\quad \boldsymbol{\varepsilon}_{t} \sim N(\mathbf{0}, \boldsymbol{\Sigma}) \quad$ com $\operatorname{vec}(\boldsymbol{\Sigma})=(0,75 ; 0,5 ; 0,5 ; 1,25)^{\prime}$. Portanto, o processo tem distribuição Gaussiana estacionária com parâmetros $\boldsymbol{\mu}=(3,05 ; 6,44)^{\prime}$ e $\operatorname{vec}(\boldsymbol{\Gamma}(0))=(1,13 ; 1,49 ; 1,49 ; 3,99)^{\prime}$, ou seja, coeficiente de correlação 0,70 . Utilizando 200 repetições com séries de tamanho 1.000, obtivemos a curva estimada, a qual apresenta comportamento praticamente constante ao longo da grade avaliada. Esta curva e a teórica apresentam-se na Figura 3.1. Além disso, calculamos o viés, o erro quadrático médio e o $p$-valor do teste de normalidade de Jarque Bera, cujos resultados apresentam-se na Tabela 3.1. Nesta, podemos observar que há uma leve subestimação da medida $\mathrm{e}$ um baixo erro quadrático médio, além de rejeição da normalidade do estimador. Na Figura 3.2, temos os histogramas das curvas estimadas, os quais indicam afastamento da normalidade.

No Apêndice C (Figura C.1), temos os resultados das simulações também para séries de tamanho $200 \mathrm{e}$ 500. Comparando as três simulações, vemos que com o aumento do tamanho da série, o viés e o erro quadrático médio diminuem, mas a rejeição da normalidade permanece inalterada (com três casas decimais).

O modelo VMA(1) estacionário considerado foi

$$
\mathbf{Z}_{t}=\boldsymbol{\Theta}_{0}-\boldsymbol{\Theta}_{1} \boldsymbol{\varepsilon}_{t-1}+\boldsymbol{\varepsilon}_{t}
$$


em que $\quad \mathbf{Z}_{t}=\left(X_{t}, Y_{t}\right)^{\prime}, \quad \boldsymbol{\Theta}_{0}=(0,4 ;-1,7)^{\prime}, \quad \operatorname{vec}\left(\boldsymbol{\Theta}_{1}\right)=(0,8 ;-0,4 ; 0,7 ; 0,6)^{\prime} \quad$ e $\quad \boldsymbol{\varepsilon}_{t} \sim N(\mathbf{0}, \boldsymbol{\Sigma}) \quad \operatorname{com}$ $\operatorname{vec}(\boldsymbol{\Sigma})=(0,75 ; 0,92 ; 0,92 ; 1,24)^{\prime}$. Então o processo tem distribuição Gaussiana estacionária com parâmetros $\boldsymbol{\mu}=(0,4 ;-1,7)^{\prime}$ e $\operatorname{vec}(\boldsymbol{\Gamma}(0))=(2,87 ; 1,39 ; 1,39 ; 1,37)^{\prime}$, resultando em correlação 0,70 . Utilizando 200 repetições de séries de tamanho 1.000, a curva de autocorrelação foi estimada, a qual apresenta-se praticamente constante conforme podemos verificar na Figura 3.3. Na Tabela 3.2 observamos a ocorrência de uma pequena subestimação da curva, baixo erro quadrático médio e rejeição da normalidade. Na Figura 3.4, vemos os histogramas os quais apresentam afastamento da normalidade.

Nas Figura C.2 do Apêndice C, apresentam-se os resultados das simulações para séries de tamanhos 200 e 500, além da anterior. Comparando estas com a anterior, observamos que com o aumento do tamanho da série, o viés geralmente diminui e o erro quadrático médio diminui, permanecendo a rejeição da normalidade.

Comparando o modelo VAR(1) com o VMA(1) avaliados acima, verificamos que este último modelo geralmente apresenta viés menor e erro quadrático médio maior para as duas amostras menores. Já para amostras de tamanho 1.000, o comportamento apresenta-se alternado. 


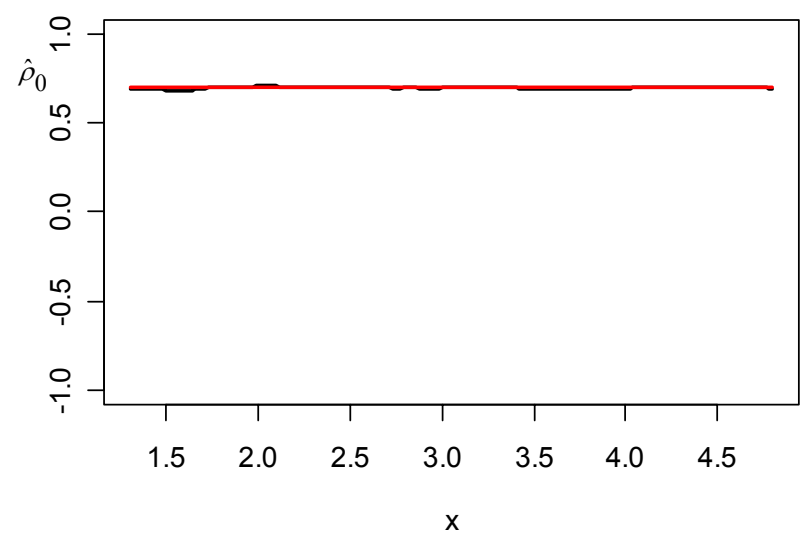

Figura 3.1 - Curva de correlação cruzada contemporânea estimada (linha) e teórica (reta), utilizando 200 séries de tamanho 1.000, observadas de um modelo VAR(1) com média $(3,05 ; 6,44)^{\prime}$, $\operatorname{vec}(\Gamma(0))=(1,13 ; 1,49 ; 1,49 ; 3,99)^{\prime}$ (correlação 0,70$)$ e inovações Gaussianas.

Tabela 3.1 - Viés, erro quadrático médio e $p$-valor do teste de normalidade de Jarque Bera de $\hat{\rho}_{0}(x)$, considerando 200 séries de tamanho 1.000 , observadas do modelo VAR(1) com média $(3,05 ; 6,44)^{\prime}$, $\operatorname{vec}(\Gamma(0))=(1,13 ; 1,49 ; 1,49 ; 3,99)^{\prime}$ (correlação 0,70$)$ e inovações Gaussianas.

\begin{tabular}{|l|r|r|r|r|r|r|r|}
\hline Grade & $\mathbf{1 , 3 0}$ & $\mathbf{1 , 8 9}$ & $\mathbf{2 , 4 7}$ & \multicolumn{1}{c|}{$\mathbf{3 , 0 5}$} & $\mathbf{3 , 6 3}$ & $\mathbf{4 , 2 2}$ & \multicolumn{1}{c|}{$\mathbf{4 , 8 0}$} \\
\hline Viés & $-0,014$ & $-0,001$ & $-0,001$ & $-0,003$ & $-0,012$ & $-0,001$ & $-0,013$ \\
\hline EQM & 0,013 & 0,008 & 0,003 & 0,003 & 0,008 & 0,005 & 0,021 \\
\hline $\boldsymbol{P}$-valor & 0,000 & 0,000 & 0,000 & 0,000 & 0,000 & 0,000 & 0,000 \\
\hline
\end{tabular}

1.3

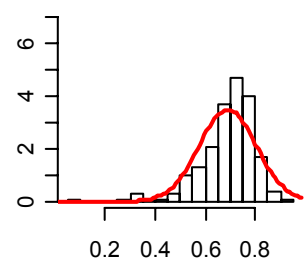

3.63

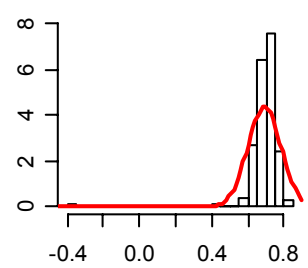

1.89

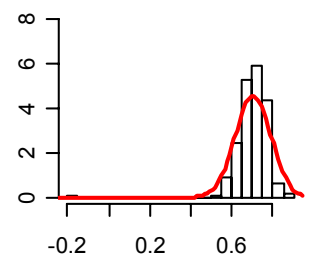

4.22

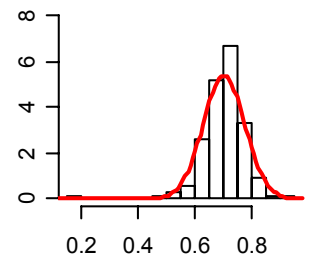

2.47

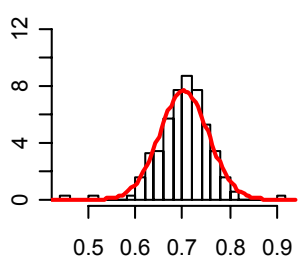

4.8

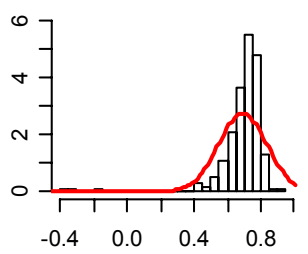

Figura 3.2 - Histogramas em pontos de grade de $\hat{\rho}_{0}(x)$, considerando 200 séries de tamanho 1.000, observadas do modelo VAR(1) com média $(3,05 ; 6,44)^{\prime}, \operatorname{vec}(\Gamma(0))=(1,13 ; 1,49 ; 1,49 ; 3,99)^{\prime}$ (correlação 0,70) e inovações Gaussianas. 


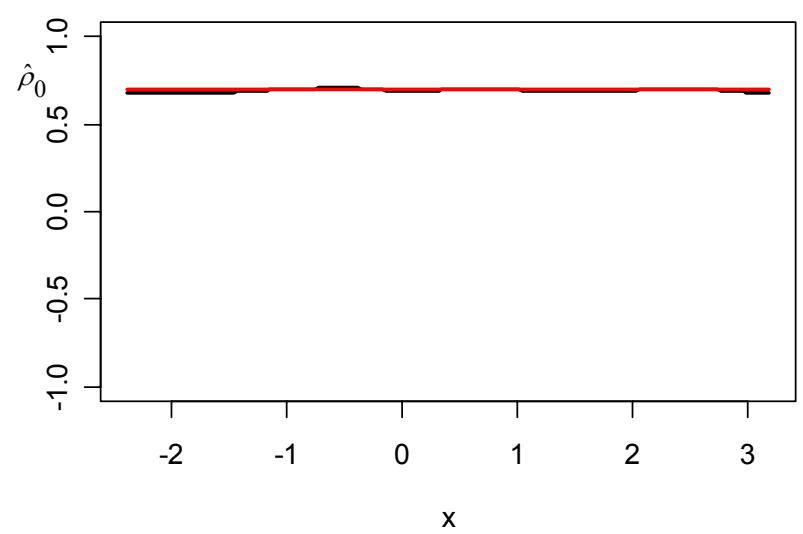

Figura 3.3 - Curva de correlação cruzada contemporânea estimada (linha) e teórica (reta), utilizando 200 séries de tamanho 1.000, observadas de um modelo VMA(1) com média $(0,4 ;-1,70)^{\prime}$, $\operatorname{vec}(\Gamma(0))=(2,87 ; 1,39 ; 1,39 ; 1,37)^{\prime}$ (correlação 0,70$)$ e inovações Gaussianas.

Tabela 3.2 - Viés, erro quadrático médio e $p$-valor do teste de normalidade de Jarque Bera de $\hat{\rho}_{0}(x)$, considerando 200 séries de tamanho 1.000 , observadas do modelo VMA(1) com média $(0,4 ;-1,70)^{\prime}$, $\operatorname{vec}(\Gamma(0))=(2,87 ; 1,39 ; 1,39 ; 1,37)^{\prime}$ (correlação 0,70$)$ e inovações Gaussianas.

\begin{tabular}{|c|c|c|c|c|c|c|c|}
\hline Grade & $-2,39$ & $-1,46$ & $-0,53$ & 0,40 & 1,33 & 2,26 & 3,19 \\
\hline Viés & $-0,022$ & $-0,017$ & 0,005 & $-0,005$ & $-0,013$ & $-0,003$ & $-0,022$ \\
\hline EQM & 0,026 & 0,014 & 0,002 & 0,004 & 0,007 & 0,005 & 0,017 \\
\hline$P$-valor & 0,000 & 0,000 & 0,000 & 0,000 & 0,000 & 0,000 & 0,000 \\
\hline
\end{tabular}

$-2.39$

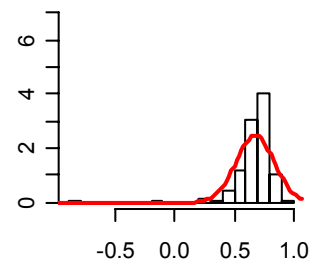

1.33

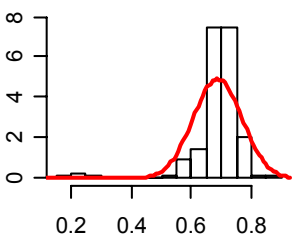

$-1.46$

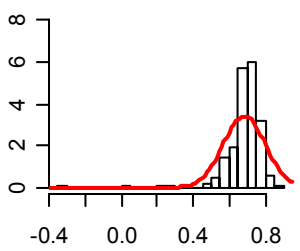

2.26

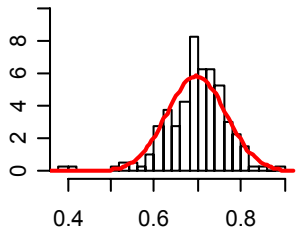

$-0.53$

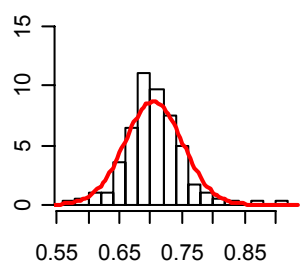

3.19

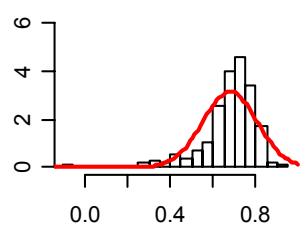

Figura 3.4 - Histogramas em pontos de grade de $\hat{\rho}_{0}(x)$, considerando 200 séries de tamanho 1.000, observadas do modelo VMA(1) com média $(0,4 ;-1,70)^{\prime}, \operatorname{vec}(\Gamma(0))=(2,87 ; 1,39 ; 1,39 ; 1,37)^{\prime}$ (correlação 0,70) e inovações Gaussiana. 


\subsubsection{Aplicações a séries reais}

A seguir analisamos a curva de correlação cruzada contemporânea considerando os retornos diários de 2/jan/95 a 27/dez/00 (1.498 dados) da Petrobrás e do Ibovespa, e também do Banespa e Ibovespa. Os parâmetros utilizados nestas simulações são os mesmos da seção anterior, porém agora com grade de 401 pontos e largura de faixa igual ao desvio padrão da série condicionada (Bjerve e Doksum, 1993).

Na seção 2.2.2 vimos que os retornos da Petrobrás $\left(X_{\mathrm{t}}\right)$ e do Ibovespa $\left(Y_{\mathrm{t}}\right)$ apresentaram correlação linear de Pearson 0,83 , função de correlação cruzada contemporânea local estimada $\left(\hat{H}_{0}\right)$ indicando forte dependência positiva entre as séries (Figura 2.38), e os gráficos com base em postos normalizados indicaram forte dependência positiva entre os valores mais altos e também entre os valores mais baixos. No gráfico (a) da Figura 3.5, observamos o gráfico de dispersão dos retornos da Petrobrás $\left(X_{\mathfrak{t}}\right)$ do Ibovespa $\left(Y_{\mathfrak{t}}\right)$. Nesta mesma figura, o gráfico (b) refere-se ao coeficiente angular local estimado o qual apresenta-se positivo, porém, com tendência decrescente até aproximadamente o valor de grade 0.03 , a partir de onde se inicia uma tendência crescente. A variância condicional local (gráfico (c)) apresenta-se maior nos extremos da grade. Por fim, a curva de correlação (gráfico (d)) que possui valores entre 0,79 e 0,87 , indica um pequeno decréscimo a partir do valor de grade -0,01, aproximadamente. Tal fato deve-se ao comportamento decrescente do coeficiente angular local. Portanto, a correlação entre os retornos da Petrobrás e do Ibovespa, passa a ser ligeiramente menor com o aumento dos possíveis valores de retorno da Petrobrás, a partir do valor $-0,01$.

As medidas de associação global (correlação linear de Pearson 0,54, por exemplo) e as medidas de dependência local verificadas na seção 2.2.2 para os retornos do Banespa $\left(X_{\mathrm{t}}\right)$ e do Ibovespa $\left(Y_{\mathrm{t}}\right)$, indicaram associação positiva e dependência local positiva entre os valores mais altos e os valores mais baixos, assim como no caso acima, porém com menor intensidade. Observando o gráfico (b) da Figura 3.6, vemos que o coeficiente angular local é positivo (mas com valores menores que o caso acima), apresentando tendência decrescente ao longo da grade. Já a variância condicional local (gráfico (c)), apresenta valores maiores nos pontos de grade maiores quando comparado com os menores. Finalmente, no gráfico (d) vemos a curva de correlação cruzada contemporânea (com valores entre 0,31 e 0,67 ) a qual apresenta tendência decrescente também a partir do ponto de grade $-0,01$, aproximadamente, porém, esta tendência é mais acentuada que no caso acima devido a maior variância nos valores de grade maiores. Ou seja, a correlação entre os retornos do Banespa e do Ibovespa passa a ser menor quanto maior for o possível valor do retorno do Banespa, a partir de $-0,01$.

A diferença de comportamento desta medida local em relação às outras medidas locais vistas na seção 2.2.2, deve-se ao fato de $\rho_{0}$ ser local em $X=x$, enquanto que as demais são locais em $X=x$ e $Y=y$. 


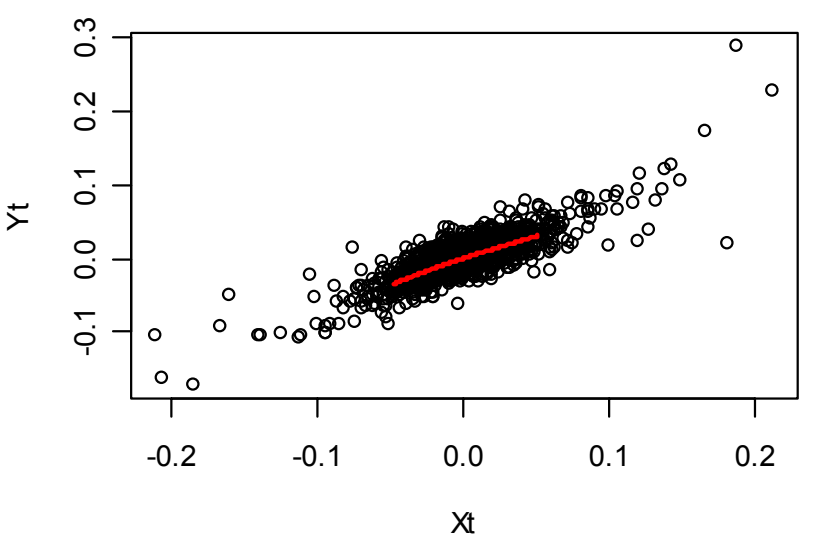

(a)

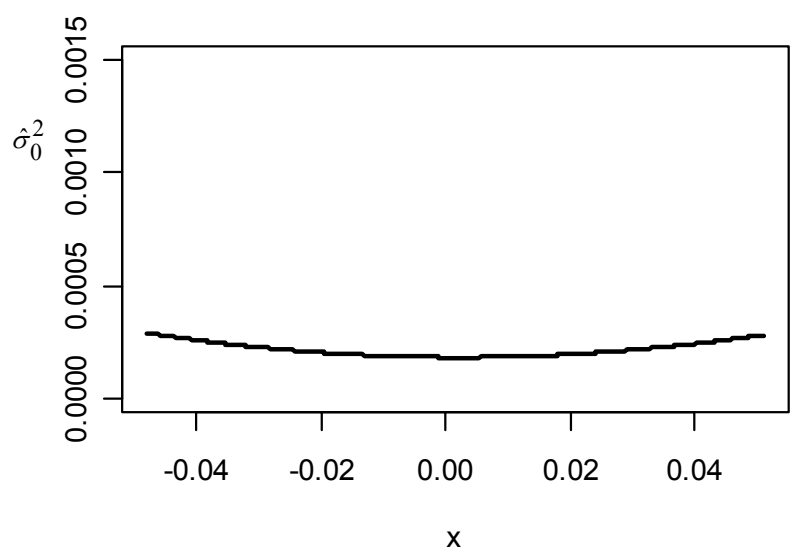

(c)

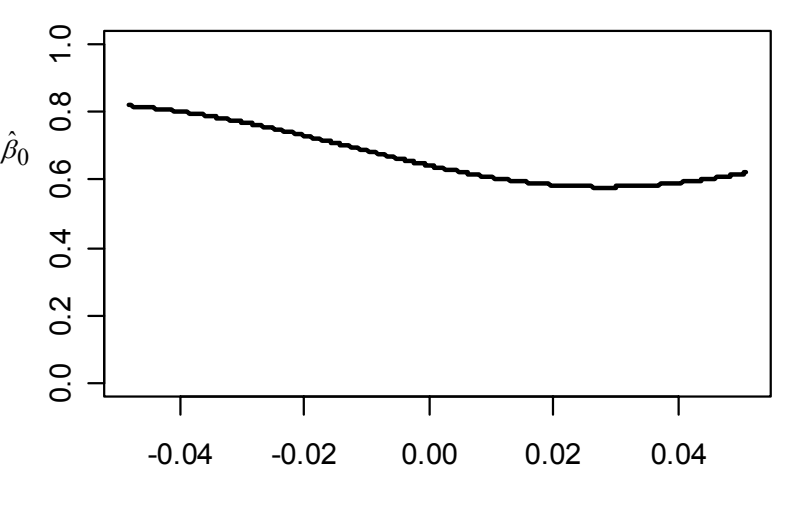

(b)

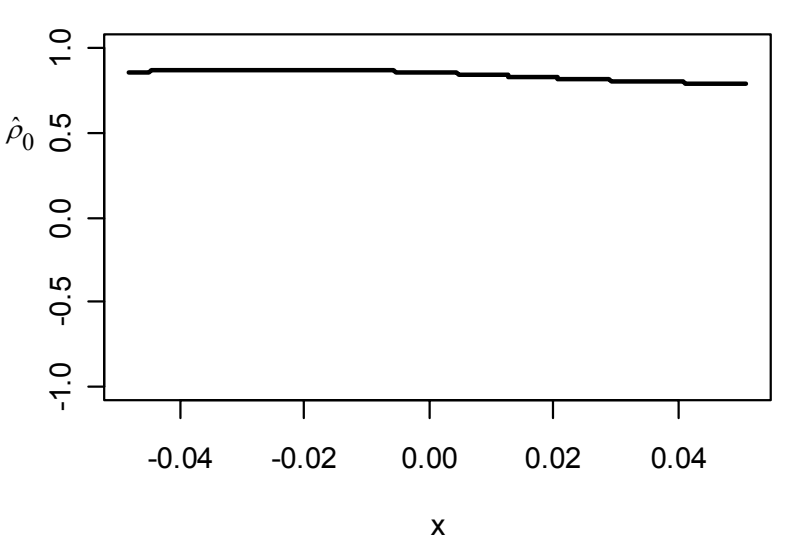

(d)

Figura 3.5 - (a) Gráfico de dispersão dos retornos do Petrobrás $\left(X_{\mathrm{t}}\right)$ e da Ibovespa $\left(Y_{\mathrm{t}}\right)$ no período de 2/jan/95 a 27/dez/00 (correlação 0,83), (b) coeficiente angular local estimado $\hat{\beta}_{0}(x)$, (c) variância condicional local estimada $\hat{\sigma}_{0}^{2}(x)$ e (d) curva de correlação cruzada contemporânea estimada $\hat{\rho}_{0}(x)$. 


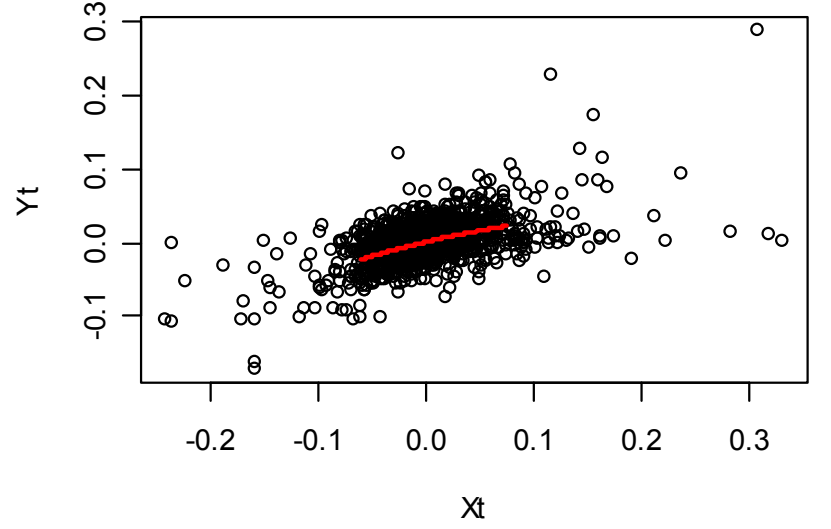

(a)

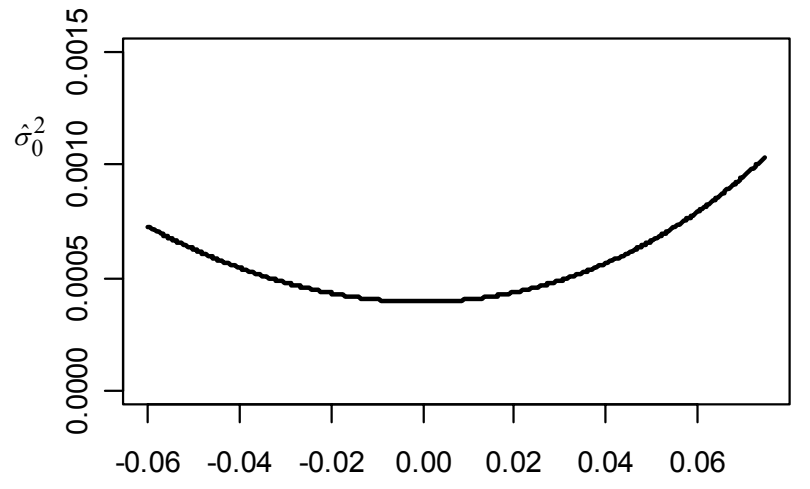

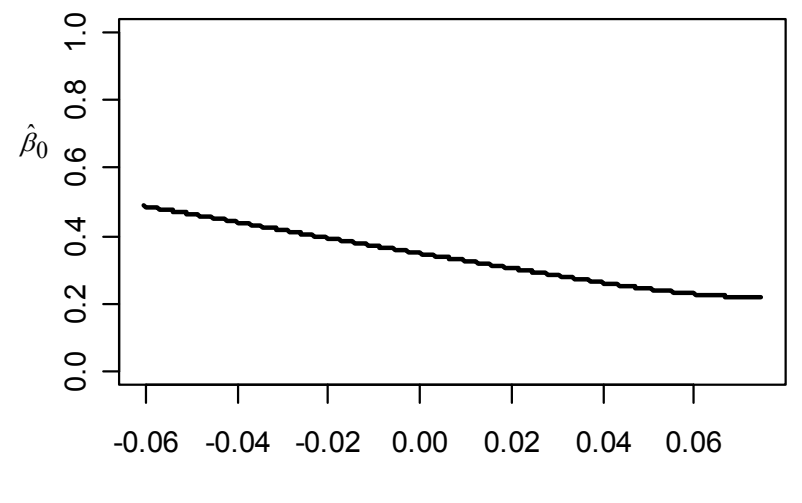

(b)

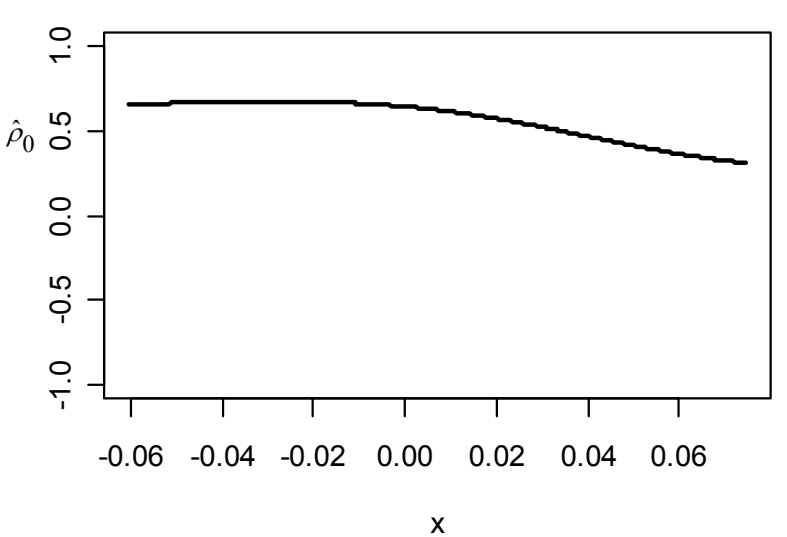

(d)

Figura 3.6 - (a) Gráfico de dispersão dos retornos do Banespa $\left(X_{\mathrm{t}}\right)$ e do Ibovespa $\left(Y_{\mathrm{t}}\right)$ no período de 2/jan/95 a 27/dez/00 (correlação 0,54), (b) coeficiente angular local estimado $\hat{\beta}_{0}(x)$, (c) variância condicional local estimada $\hat{\sigma}_{0}^{2}(x)$ e (d) curva de correlação cruzada contemporânea estimada $\hat{\rho}_{0}(x)$. 


\subsection{Curva de Autocorrelação}

Considere o seguinte modelo utilizado em séries financeiras:

$$
X_{t}=m\left(X_{t-1}, \ldots, X_{t-p}\right)+\sigma\left(X_{t-1}, \ldots, X_{t-p}\right) \varepsilon_{t}, \varepsilon_{t} \sim \operatorname{iid}(0,1) \text { e } \varepsilon_{t} \perp X_{t-k}, k \geq 1,
$$

em que $m\left(\right.$.) e $\sigma^{2}$ (.) representam, respectivamente, a esperança e a variância condicional de $X_{t}$ dado $X_{t-1}, \ldots, X_{t-p}$. Este modelo pode ser denominado CHARN (de "conditional heteroscedastic autoregressive nonlinear" segundo Yang et al., 1999) ou modelo tipo ARCH (Masry e Tjøstheim, 1995).

Se utilizarmos modelos paramétricos para estimar as funções $m($.$) e \sigma($.$) , temos que um modelo$ particular é o ARCH. Para ver isto, considere $m\left(\right.$.) linear e $\sigma^{2}\left(X_{t-1}, \ldots, X_{t-p}\right)=\alpha_{0}+\alpha_{1} X_{t-1}^{2}+\ldots+\alpha_{p} X_{t-p}^{2}$, então, o modelo resultante é o $\mathrm{ARCH}(\mathrm{p})$ definido por Engle (1982). Outra abordagem é utilizar técnicas não paramétricas para estimar estas funções, e então este modelo pode ser denominado NARCH (de "nonparametric autoregressive conditional heteroscedastic", conforme Fan e Yao, 2005).

Sob certas condições no crescimento de $m($.$) e \sigma\left(\right.$.), o processo $\left\{X_{\mathrm{t}}\right\}$ gerado pelo modelo

$$
X_{t+i_{p+1}}=m\left(X_{t+i_{1}}, \ldots, X_{t+i_{p}}\right)+\sigma\left(X_{t+i_{1}}, \ldots, X_{t+i_{p}}\right) \varepsilon_{t+i_{p+1}}, 0 \leq i_{1}<i_{2}<\ldots<i_{p+1},
$$

(que é um modelo que possibilita uma quantidade menor de variáveis aleatórias defasadas para a mesma ordem $p$ do modelo anterior) é estritamente estacionário e $\alpha$-mixing, com coeficiente mixing apresentando decaimento exponencial (Masry e Tjøstheim, 1995 - Lema 3.1).

Observamos que um processo $\operatorname{GARCH}(\mathrm{p}, \mathrm{q})$ representado por $X_{t}=\sigma_{t} \varepsilon_{t}$, $\sigma_{t}^{2}=\alpha_{0}+\sum_{i=1}^{p} \alpha_{i} X_{t-i}^{2}+\sum_{j=1}^{q} \beta_{j} \sigma_{t-j}^{2}$, em que $\varepsilon_{t} \sim \operatorname{IID}(0,1), \alpha_{i} \geq 0, \beta_{j} \geq 0$, é $\alpha$-mixing com coeficiente mixing apresentando decaimento exponencial se (i) $\sum_{i=1}^{p} \alpha_{i}+\sum_{j=1}^{q} \beta_{j}<1$ e (ii) a função de densidade de $\varepsilon_{t}$ é positiva num intervalo contendo o zero (Fan e Yao, 2005 - página 70).

Sabemos que se $m($.) é linear e $\sigma($.) é constante, então recaímos no modelo AR(p). E se o processo é Gaussiano estacionário com média zero, então $m($.$) é linear e \sigma($.$) é constante (Fan e Yao, 2005). Além$ disso, um processo linear usual é $\alpha$-mixing com coeficiente mixing apresentando decaimento geométrico (Bosq, 1998 - páginas 18 e 46).

A partir de agora, iremos nos concentrar na subclasse dos sistemas não lineares tipo ARCH em que $m($. e $\sigma($.$) são funções de uma única variável aleatória defasada.$

Seja $\left\{X_{t}, t \in Z\right\}$ um processo estacionário estrito e de segunda ordem a valores contínuos, que pode ser considerado gerado a partir do modelo (3.1) em que $Y_{t}=X_{t+\tau}$, ou seja

$$
X_{t+\tau}=m\left(X_{t}\right)+\sigma\left(X_{t}\right) \varepsilon_{t+\tau}, \operatorname{com} \varepsilon_{t} \sim \operatorname{iid}(0,1) \text { e } \varepsilon_{t+k} \perp X_{t}, k \geq 1,
$$

sendo que $m(x)=E\left[X_{t+\tau} / X_{t}=x\right]$ e $\sigma^{2}(x)=\operatorname{Var}\left[X_{t+\tau} / X_{t}=x\right]$ estão bem definidas.

A seguir, vamos derivar a expressão da curva de correlação (1.2).

Como o processo é estacionário estrito, vimos na seção 2.1 que a distribuição de $\left(X_{t}, X_{t+\tau}\right)$ só depende da defasagem $\tau$, e então $\beta(x)=\partial / \partial x E\left[X_{t+\tau} / X_{t}=x\right]$ e $\sigma^{2}(x)=\operatorname{Var}\left[X_{t+\tau} / X_{t}=x\right]$ também só dependem da defasagem $\tau$. 
Portanto, a curva de correlação (1.2) pode ser escrita da seguinte forma

$$
\rho_{\tau}(x)=\frac{\beta_{\tau}(x) \sqrt{\gamma_{0}}}{\sqrt{\beta_{\tau}^{2}(x) \gamma_{0}+\sigma_{\tau}^{2}(x)}}, \forall x \in S \operatorname{com} f(x)>0, \forall t, \tau \in Z,
$$

em que $\beta_{\tau}(x)$ e $\sigma_{\tau}^{2}(x)$ são como acima, e $\gamma_{0}=\operatorname{Var}\left[X_{t}\right]$.

Denominamos esta medida local de curva de autocorrelação.

As seguintes propriedades são válidas para a curva de autocorrelação (3.5):

(i) $-1 \leq \rho_{\tau}(x) \leq+1, \forall \tau \in Z, \forall x \in S$;

prova:

Para a situação em que a variância condicional é nula (veja a propriedade a seguir), temos que $\rho_{\tau}(x)=1 \times \operatorname{sinal}(x)$. Caso contrário, como $\rho_{\tau}(x)$ pode ser reescrita como $\rho_{\tau}(x)=\sqrt{\beta_{\tau}^{2}(x) \gamma_{0} /\left(\beta_{\tau}^{2}(x) \gamma_{0}+\sigma_{\tau}^{2}(x)\right)}$, então é imediato que $-1 \leq \rho_{\tau}(x) \leq+1$.

(ii) $\rho_{\tau}(x)=1 \times \operatorname{sinal}(x)$ se e somente se $\tau=0, \forall x \in S$, se o processo é não constante; prova:

Se $\tau=0$, então $E\left[X_{t} / X_{t}=x\right]=x$ e $\beta_{0}(x)=1 \times \operatorname{sinal}(x)$. Além disso, $\sigma_{0}^{2}(x)=\operatorname{Var}\left[X_{t} / X_{t}=x\right]=0$. Portanto, $\rho_{0}(x)=\operatorname{sinal}(x)$. Se $\rho_{\tau}(x)= \pm 1, \forall x \in S$, então $\sigma_{\tau}^{2}(x)=0$, que ocorre somente quando $\tau=0$.

(iii) $\rho_{-\tau}(x)=\rho_{\tau}(x), \forall \tau \in Z, \forall x \in S$;

prova:

Como o processo é estacionário estrito, então $f_{X_{t}, X_{t+\tau}}\left(x_{1}, x_{2}\right)=f_{X_{t-\tau}, X_{t}}\left(x_{1}, x_{2}\right)$ que implica em $E\left[X_{t+\tau} / X_{t}=x\right]=E\left[X_{t} / X_{t-\tau}=x\right]$ e $\operatorname{Var}\left[X_{t+\tau} / X_{t}=x\right]=\operatorname{Var}\left[X_{t} / X_{t-\tau}=x\right]$. Portanto, $\rho_{\tau}(x)=\rho_{-\tau}(x)$.

(iv) $\rho_{\tau}(x)=\rho_{\tau}, \forall \tau \in Z, \forall x \in S$ se $\left\{X_{t}\right\}$ é um processo Gaussiano.

prova:

Se o processo é Gaussiano, então $X_{t+\tau} / X_{t}=x \sim N\left(\mu+\rho_{\tau}(x-\mu), \gamma_{0}\left(1-\rho_{\tau}^{2}\right)\right), \quad \forall t, \tau \in Z$. Portanto, $\beta_{\tau}(x)=\rho_{\tau}$, e então

$$
\rho_{\tau}(x)=\frac{\beta_{\tau}(x) \sqrt{\gamma_{0}}}{\sqrt{\beta_{\tau}^{2}(x) \gamma_{0}+\sigma_{\tau}^{2}(x)}}=\frac{\rho_{\tau} \sqrt{\gamma_{0}}}{\sqrt{\rho_{\tau}^{2} \gamma_{0}+\gamma_{0}\left(1-\rho_{\tau}^{2}\right)}}=\rho_{\tau} .
$$

A seguir encontram-se as expressões de $\rho_{\tau}(x), \forall x \in S$ e $\forall \tau \in Z$, para alguns modelos simples.

\section{(i) Modelo AR(1)}

Considere um processo $\left\{X_{t}, t \in Z\right\}$ estacionário estrito e de segunda ordem, seguindo um modelo $\operatorname{AR}(1)$ representado por $X_{t}=\phi_{0}+\phi_{1} X_{t-1}+a_{t}, a_{t} \sim i i d\left(0, \sigma_{a}^{2}\right)$. Pode-se verificar que $\rho_{\tau}(x)=\rho_{\tau}, \forall x \in S, \tau \in Z$ (veja prova no Apêndice $\mathrm{B}$ ). Portanto, neste modelo, a curva de autocorrelação reduz-se à função de autocorrelação. 


\section{(ii) Modelo MA(1)}

Considere um processo $\left\{X_{t}, t \in Z\right\}$ estacionário estrito e de segunda ordem, seguindo um modelo MA(1) com a seguinte representação: $X_{t}=\theta_{0}-\theta_{1} a_{t-1}+a_{t}, a_{t} \sim \operatorname{iid}\left(0, \sigma_{a}^{2}\right)$. A curva de autocorrelação para este modelo é dada por (veja Apêndice B):

$$
\rho_{\tau}(x)= \begin{cases}1 & , \tau=0 \\ -\sqrt{\frac{\theta_{1}^{2}\left(1+\theta_{1}^{2}\right)}{2 \theta_{1}^{4}+\theta_{1}^{2}+1}} & ,|\tau|=1, \quad \forall x \in S, \\ 0 & ,|\tau| \geq 2\end{cases}
$$

que é constante em relação a $x$.

\section{(iii) Modelo ARCH(1)}

Considere um processo $\left\{X_{t}, t \in Z\right\}$ estacionário estrito e de segunda ordem, seguindo um modelo $\mathrm{ARCH}(1)$ representado por $X_{t}=\sigma_{t} \varepsilon_{t}, \sigma_{t}^{2}=\alpha_{0}+\alpha_{1} X_{t-1}^{2}$ e $\varepsilon_{t} \sim \operatorname{iid}(0,1)$. Como neste modelo a componente da esperança condicional é nula, então a derivada desta em relação a $x$ (coeficiente angular) também será nula. Portanto, a curva de autocorrelação será nula.

Para o processo em estudo, sugerimos um estimador similar ao estimador (3.3), em que consideramos $Y_{t}=X_{t+\tau}, t=1, \ldots, T-\tau, \tau=0, \ldots, T-1$.

Portanto, observados $\left(X_{1}, \ldots, X_{T}\right)$, a curva de autocorrelação (3.5) pode ser estimada através de:

$$
\hat{\rho}_{\tau}(x)=\frac{\hat{\beta}_{\tau}(x) \sqrt{c_{0}}}{\sqrt{\hat{\beta}_{\tau}^{2}(x) c_{0}+\hat{\sigma}_{\tau}^{2}(x)}}
$$

em que

$c_{0}=\hat{\gamma}_{0}=\frac{1}{T} \sum_{t=1}^{T}\left(X_{i}-\bar{X}\right)^{2}$,

$\hat{\beta}_{\tau}(x)=\hat{m}^{(1)}(x)$ utilizando um polinômio quadrático local com kernel $K_{l}$, largura de faixa $h_{1}$ e o conjunto de dados $\left\{\left(X_{t}, X_{t+\tau}\right), t=1, \ldots, T-\tau\right\}$,

$\hat{\sigma}_{\tau}^{2}(x)=\hat{m}^{(0)}(x)$ utilizando um polinômio linear local, um kernel $K_{2}$, uma largura de faixa $h_{2}$ e o conjunto de dados $\left\{\left(X_{t}, \hat{r}_{t}^{2}\right), t=1, \ldots, T-\tau\right\}$, sendo $\hat{r}_{t}=X_{t+\tau}-\hat{m}\left(X_{t}\right)$ com $\hat{m}\left(X_{t}\right)$ sendo previsto após o ajuste de um polinômio linear local nos pontos de grade $x \in G$, que utiliza o kernel $K_{l}$, a largura de faixa $h_{1}$ e o conjunto de dados $\left\{\left(X_{t}, X_{t+\tau}\right), t=1, \ldots, T-\tau\right\}$.

Considere as condições de regularidade (C1) a (C12) da seção 3.1.4 devidamente adptadas.

Teorema 3.2. Considere um processo $\left\{X_{t}, t \in Z\right\}$ estritamente estacionário e de segunda ordem. Satisfeitas as condições (C1) a (C12), então

$$
\hat{\rho}_{\tau}(x) \underset{\mathrm{T} \rightarrow \infty}{\stackrel{P}{\longrightarrow}} \rho_{\tau}(x) \text {, para cada } x \in S \text { fixado. }
$$

prova: Similar à do Teorema 3.1 . 


\subsubsection{Simulações}

Avaliamos o comportamento da curva de autocorrelação utilizando o modelo AR(1)-ARCH(1) dado por

$$
\begin{aligned}
& X_{t}=\phi_{1} X_{t-1}+a_{t}, \\
& a_{t}=\sigma_{t} \varepsilon_{t} \\
& \sigma_{t}^{2}=\alpha_{0}+\alpha_{1} a_{t-1}^{2},
\end{aligned}
$$

em que $\varepsilon_{t} \sim N(0,1), \phi_{0}=0, \phi_{1}=0,8, \alpha_{0}=0,1$ e $\alpha_{1}=0,8$. Para tanto, utilizamos 200 repetições de séries de tamanho 200, 500 e 1.000. A grade, o percentual de dados e a largura de faixa utilizados foram iguais aos da seção 3.1.5.

Referente a simulação com séries de tamanho 1.000, observamos na Figura 3.11 a curva de autocorrelação média estimada para os lags 1 a 4. Com o aumento do lag, vemos que a curva de autocorrelação diminui de intensidade (possivelmente devido à redução da dependência da parte autoregressiva do modelo) e torna-se mais linear (devido à suavização da componente da volatidade do modelo). Na Tabela 3.4, vemos que com o aumento da defasagem, a variância do estimador aumenta e a rejeição da normalidade permanece inalterada. Na Figura 3.12, apresentam-se histogramas do estimador no lag 2 em alguns pontos de grade, os quais indicam afastamento da normalidade.

A variância do estimador diminui com o aumento da defasagem, enquanto que o nível descritivo (nulo) permanece inalterado, conforme podemos observar através da comparação da simulação anterior com as simulações contendo séries de tamanho 200 e 500 (Figura C.3 do Apêndice C).

Tabela 3.3 - Viés, erro quadrático médio e $p$-valor do teste de normalidade de Jarque Bera de $\hat{\rho}_{\tau}(x)$, considerando 200 séries de tamanho 1.000, observadas do modelo AR(1)-ARCH(1), com inovações Gaussianas.

\begin{tabular}{|l|c|c|c|c|c|c|c|c|}
\hline Medidas & $\boldsymbol{L a g}$ & $\mathbf{- 1 , 5 3}$ & $\mathbf{- 1 , 0 2}$ & $\mathbf{- 0 , 5 1}$ & $\mathbf{0 , 0 0}$ & $\mathbf{0 , 5 1}$ & $\mathbf{1 , 0 2}$ & $\mathbf{1 , 5 3}$ \\
\hline Variância & $\mathbf{1}$ & 0,042 & 0,008 & 0,005 & 0,002 & 0,009 & 0,039 & 0,080 \\
\hline Variância & $\mathbf{2}$ & 0,066 & 0,055 & 0,034 & 0,013 & 0,026 & 0,050 & 0,101 \\
\hline Variância & $\mathbf{3}$ & 0,095 & 0,091 & 0,037 & 0,017 & 0,032 & 0,056 & 0,120 \\
\hline Variância & $\mathbf{4}$ & 0,136 & 0,084 & 0,055 & 0,038 & 0,041 & 0,095 & 0,124 \\
\hline $\boldsymbol{P}$-valor & $\mathbf{1}$ & 0,000 & 0,000 & 0,000 & 0,000 & 0,000 & 0,000 & 0,000 \\
\hline $\boldsymbol{P}$-valor & $\mathbf{2}$ & 0,000 & 0,000 & 0,000 & 0,000 & 0,000 & 0,000 & 0,000 \\
\hline $\boldsymbol{P}$-valor & $\mathbf{3}$ & 0,000 & 0,000 & 0,000 & 0,000 & 0,000 & 0,000 & 0,000 \\
\hline $\boldsymbol{P}$-valor & $\mathbf{4}$ & 0,000 & 0,000 & 0,000 & 0,000 & 0,000 & 0,000 & 0,000 \\
\hline
\end{tabular}


tau=1

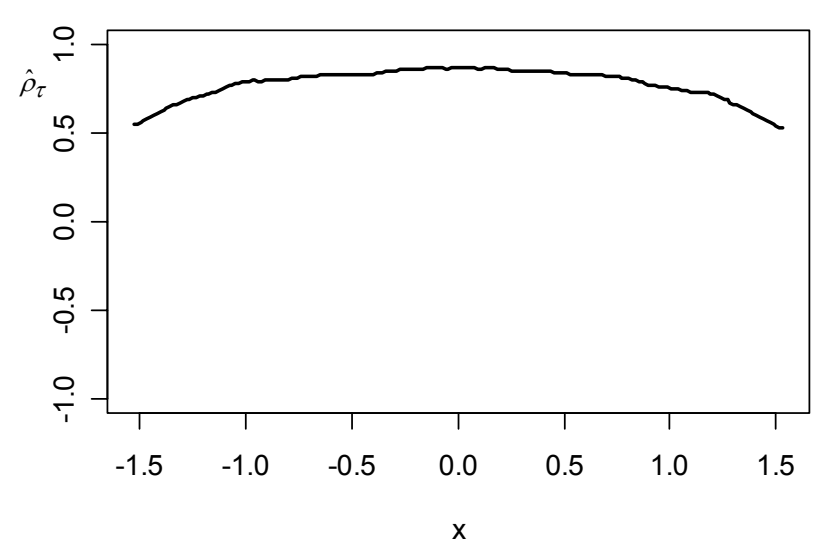

tau=3

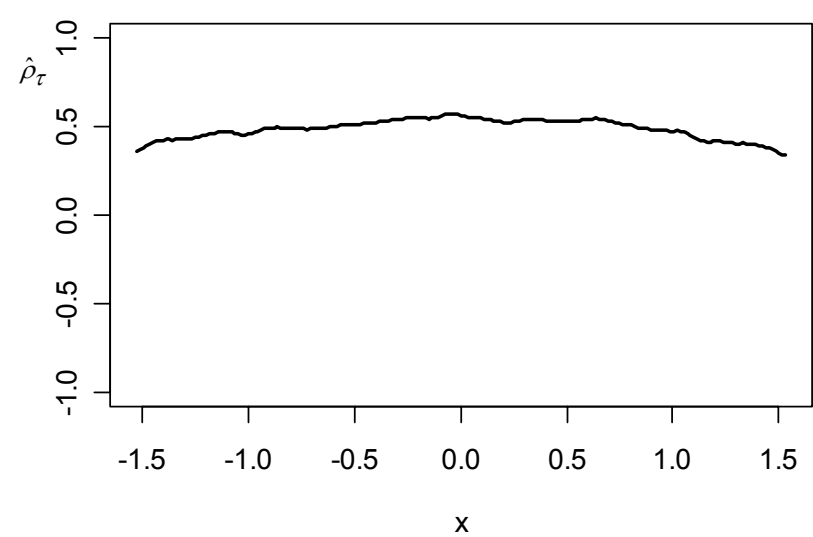

tau=2

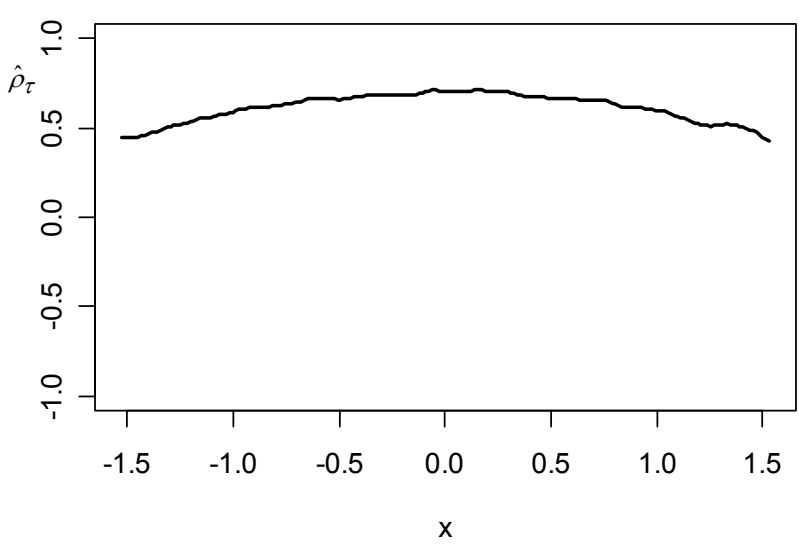

$\operatorname{tau}=4$

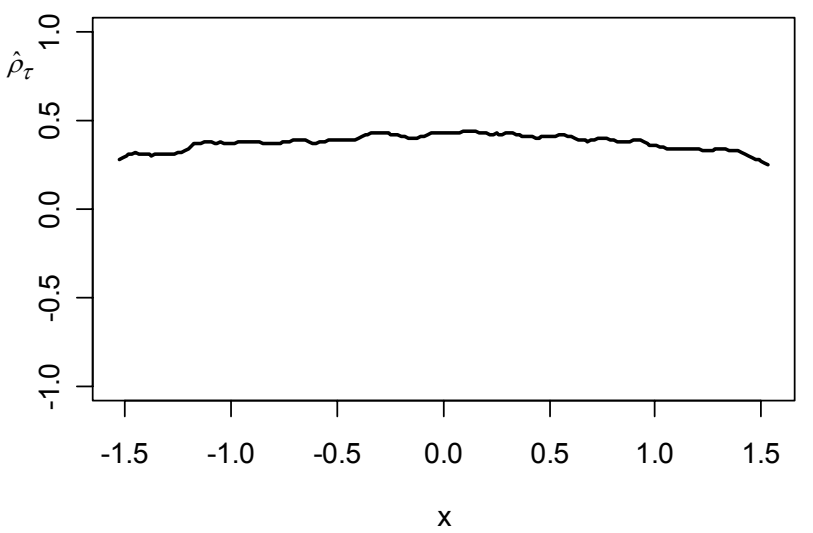

Figura 3.7 - Curva de autocorrelação média para os lags 1 a 4, considerando 200 séries de tamanho 1.000, observadas de um modelo $\mathrm{AR}(1)-\mathrm{ARCH}(1) \operatorname{com} \phi_{0}=0, \phi_{1}=0,8, \alpha_{0}=0,1$ e $\alpha_{1}=0,8$, e inovações Gaussianas. 
$-1.16$

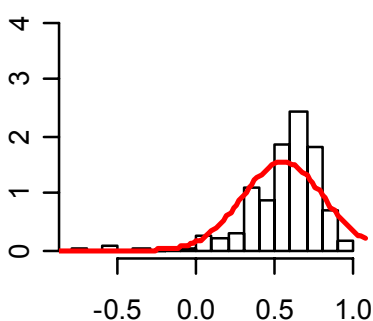

0.39

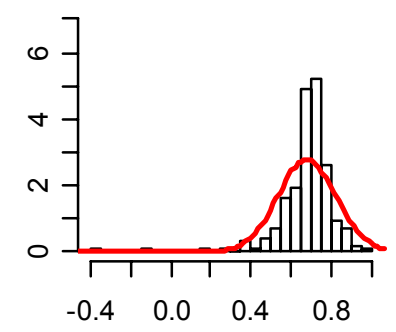

$-0.78$

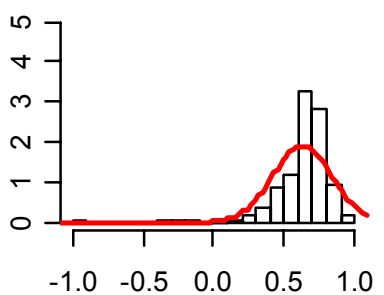

0.78

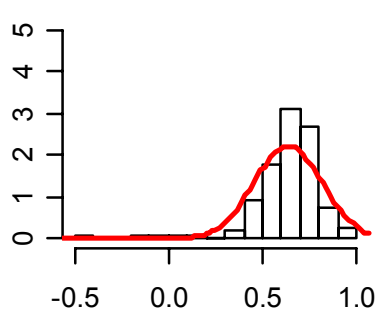

$-0.39$

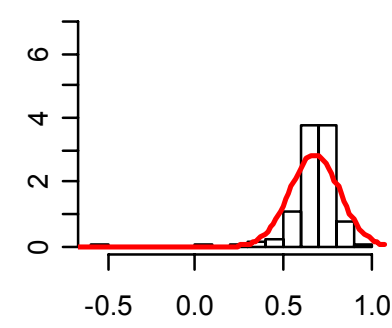

1.16

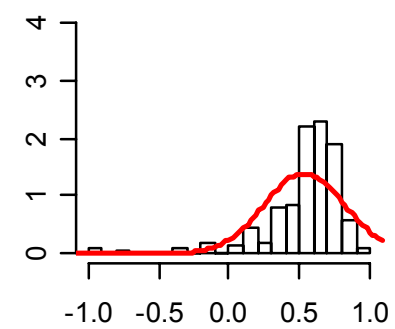

Figura 3.8 - Histogramas em pontos de grade de $\hat{\rho}(x)$ para o lag 2, considerando 200 séries de tamanho 1.000, observadas do modelo $\operatorname{AR}(1)-\operatorname{ARCH}(1) \operatorname{com} \phi_{0}=0, \phi_{1}=0,8, \alpha_{0}=0,1$ e $\alpha_{1}=0,8$, e inovações Gaussianas. 


\subsubsection{Aplicações a séries reais}

A seguir analisamos a curva de autocorrelação considerando os retornos diários da Petrobrás e do Banespa (2/jan/95 a 27/dez/00), considerando a série adiantada em 1, 2 ou 3 dias dado a série. Os parâmetros utilizados nestas simulações são os mesmos da seção 3.1.6.

Para os retornos da Petrobrás, para o lag 1, vemos no último gráfico da Figura 3.9 que há indicações da correlação local positiva apresentar inicialmente uma tendência crescente, seguida de uma leve tendência decrescente. Já a curva de autocorrelação no lag 2 (gráfico (d) da Figura 3.10) apresenta inicialmente valores negativos e com uma leve tendência crescente, permanecendo constante e positiva para os valores de grade próximos de zero, e então ocorre uma tendência decrescente atingindo novamente valores negativos. Por fim, para a defasagem 3, vemos que a curva de autocorrelação (último gráfico da Figura 3.11) apresenta uma suave oscilação em torno do valor zero ao longo da grade.

Nas figuras 3.12 a 3.14, para as defasagens 1 a 3, respectivamente, apresentam-se as curvas de autocorrelação (gráfico (d)), dentre outros, para os retornos diários do Banespa. No lag 1, a curva de autocorrelação positiva apresenta uma leve tendência crescente para os maiores valores da grade. Já no lag 2, a correlação local apresenta inicialmente valores negativos alcançando valores positivos, pois há tendência crescente a partir de valores de grade $-0,01$ aproximadamente. A curva de autocorrelação apresenta-se praticamente constante no lag 3 . 


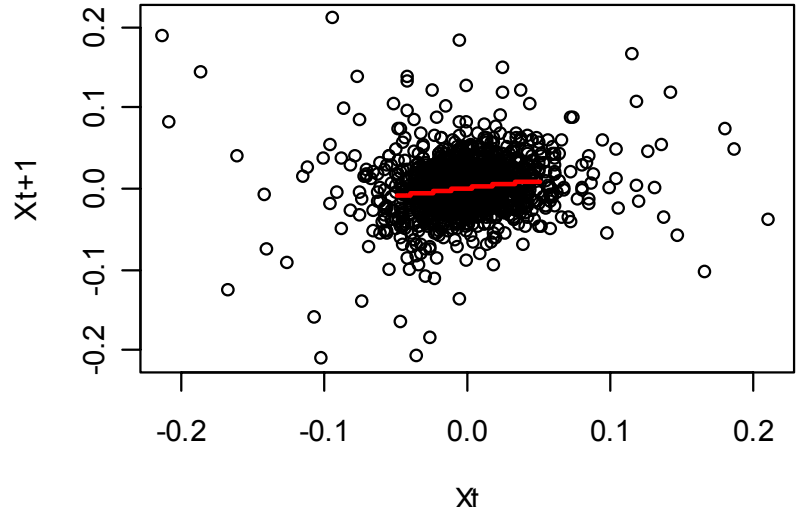

(a)

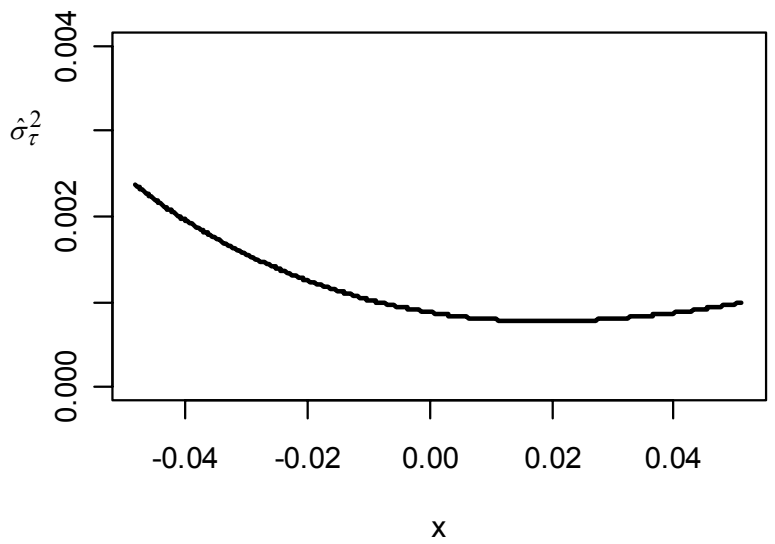

(c)

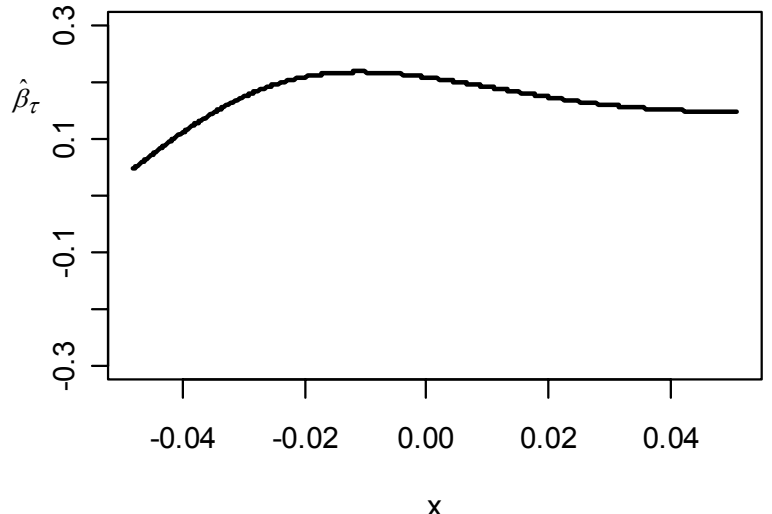

(b)

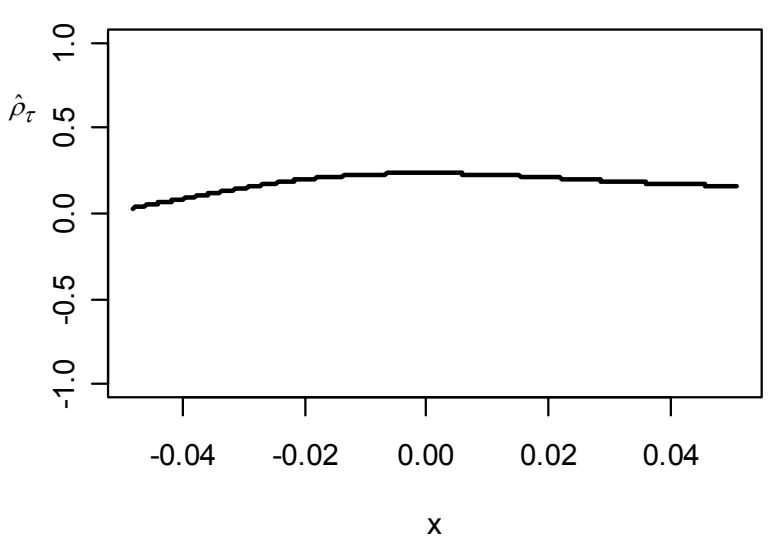

(d)

Figura 3.9 - Para os retornos da Petrobrás $\left(X_{\mathrm{t}}\right)$ no período de $2 /$ jan/95 a 27/dez/00 com defasagem 1 (correlação 0,111), temos: (a) gráfico de dispersão, (b) coeficiente angular local estimado $\hat{\beta}_{\tau}(x)$, (c) variância condicional local estimada $\hat{\sigma}_{\tau}^{2}(x)$, e (d) curva de autocorrelação estimada $\hat{\rho}_{\tau}(x)$. 


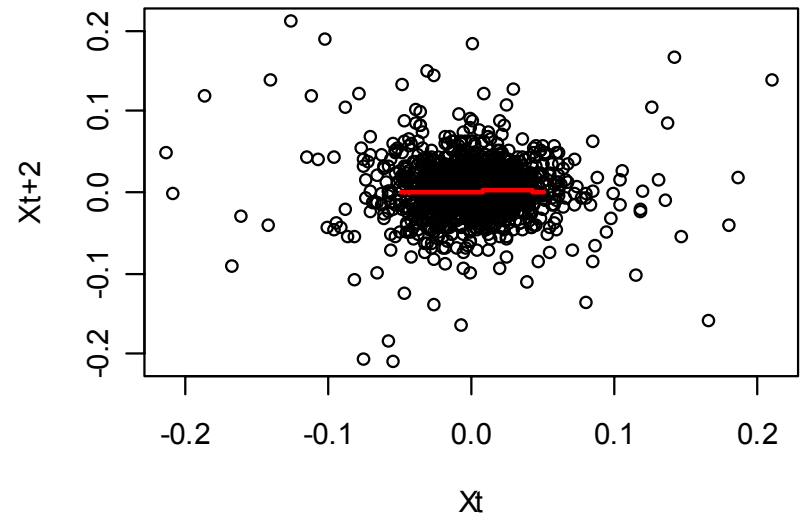

(a)

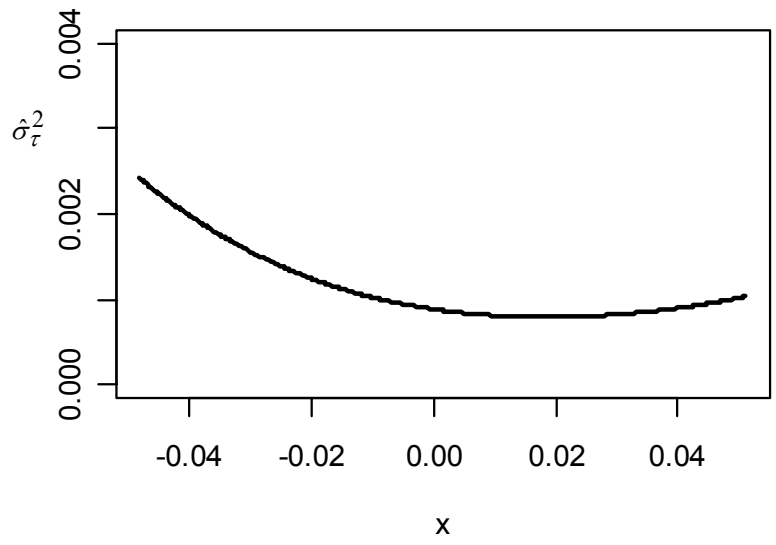

(c)

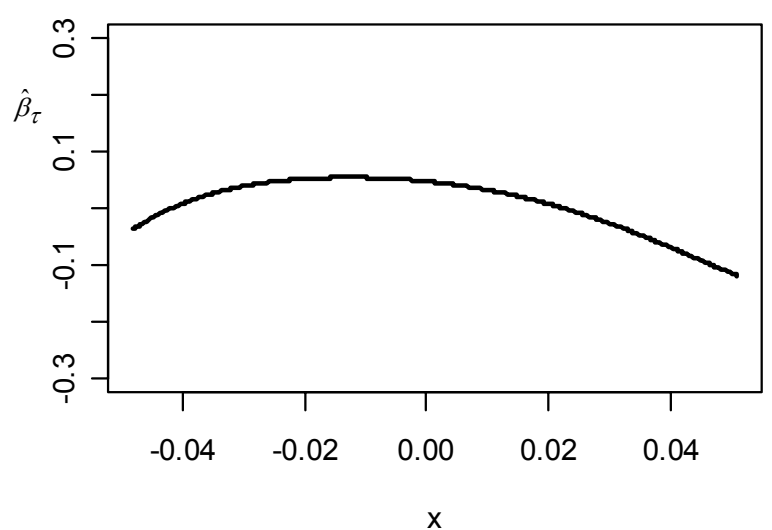

(b)

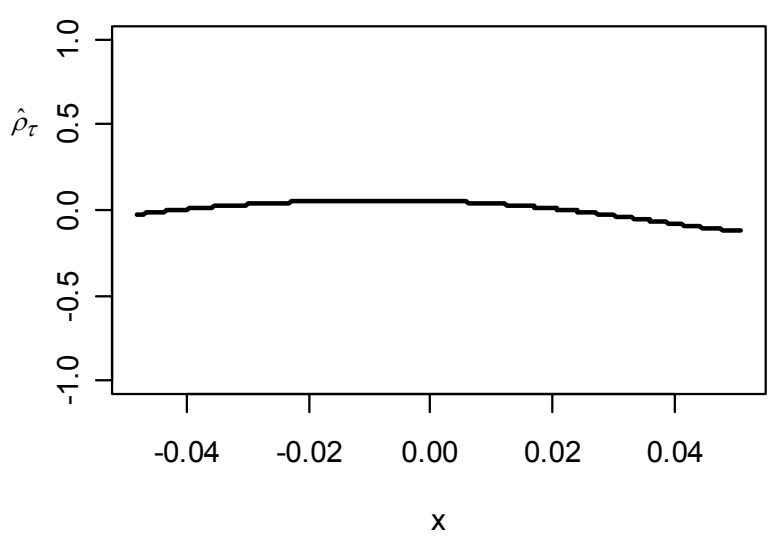

(d)

Figura 3.10 - Para os retornos da Petrobrás $\left(X_{\mathrm{t}}\right)$ no período de 2/jan/95 a 27/dez/00 com defasagem 2 (correlação -0,020), temos: (a) gráfico de dispersão,

(b) coeficiente angular local estimado $\hat{\beta}_{\tau}(x)$, (c) variância condicional local estimada $\hat{\sigma}_{\tau}^{2}(x)$, e (d) curva de autocorrelação estimada $\hat{\rho}_{\tau}(x)$. 


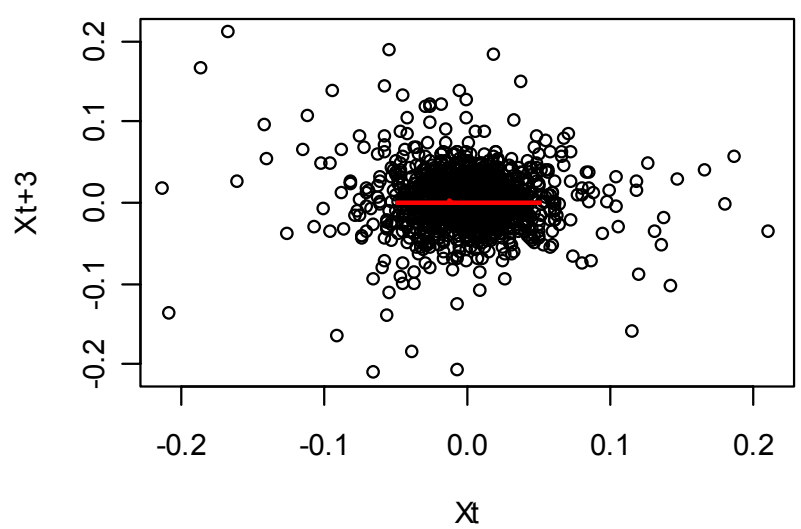

(a)

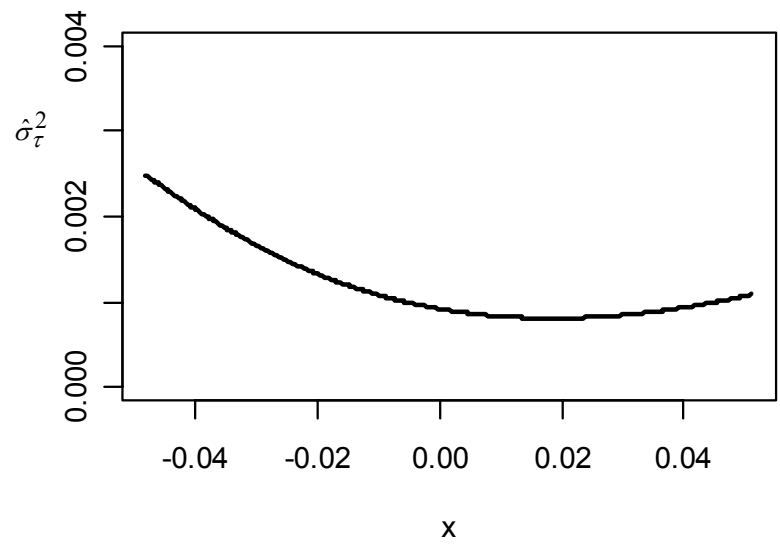

(c)

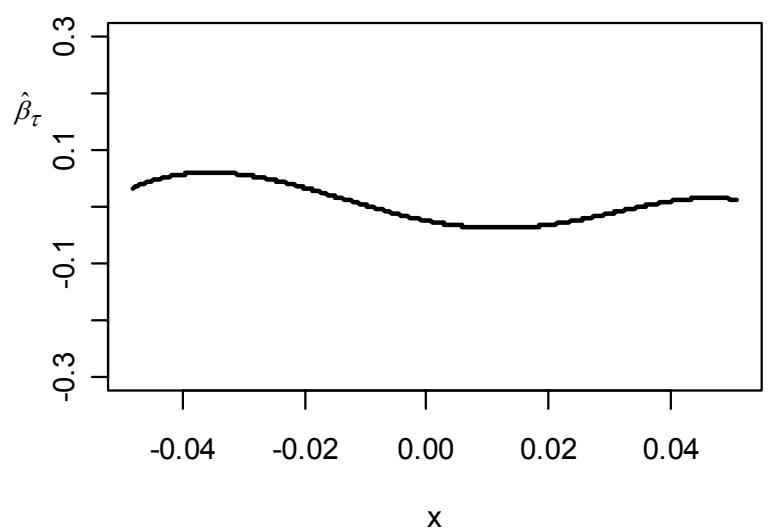

(b)

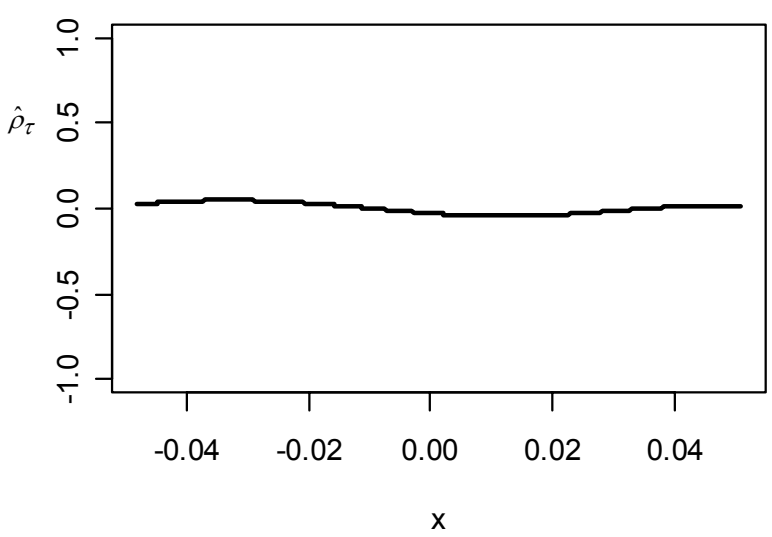

(d)

Figura 3.11 - Para os retornos da Petrobrás $\left(X_{\mathfrak{t}}\right)$ no período de 2/jan/95 a 27/dez/00 com defasagem 3 (correlação -0,065), temos: (a) gráfico de dispersão (b) coeficiente angular local estimado $\hat{\beta}_{\tau}(x)$, (c) variância condicional local estimada $\hat{\sigma}_{\tau}^{2}(x)$, e (d) curva de autocorrelação estimada $\hat{\rho}_{\tau}(x)$. 


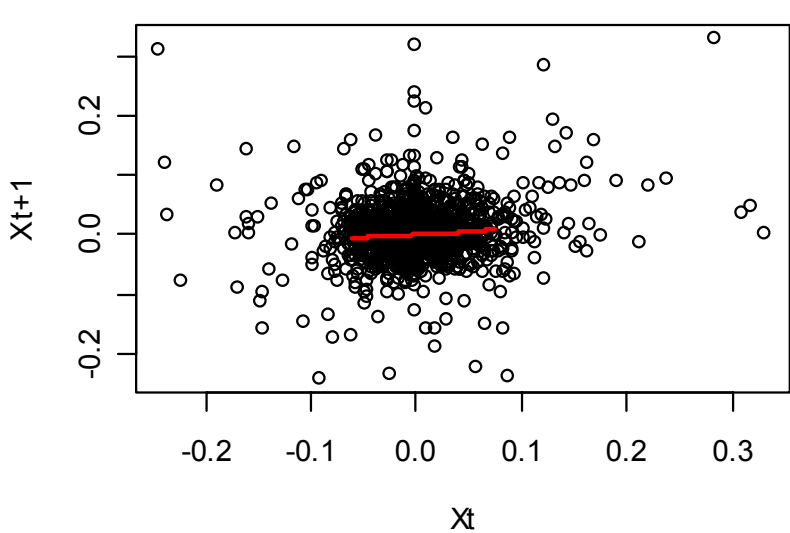

(a)

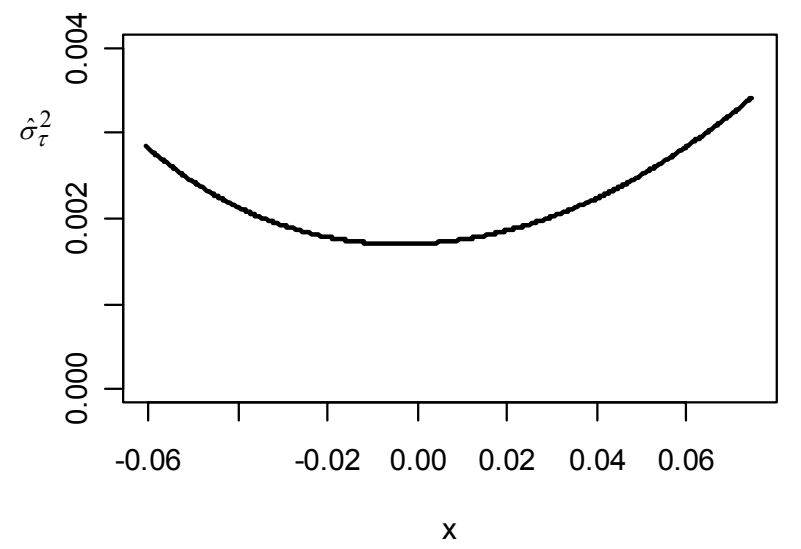

(c)

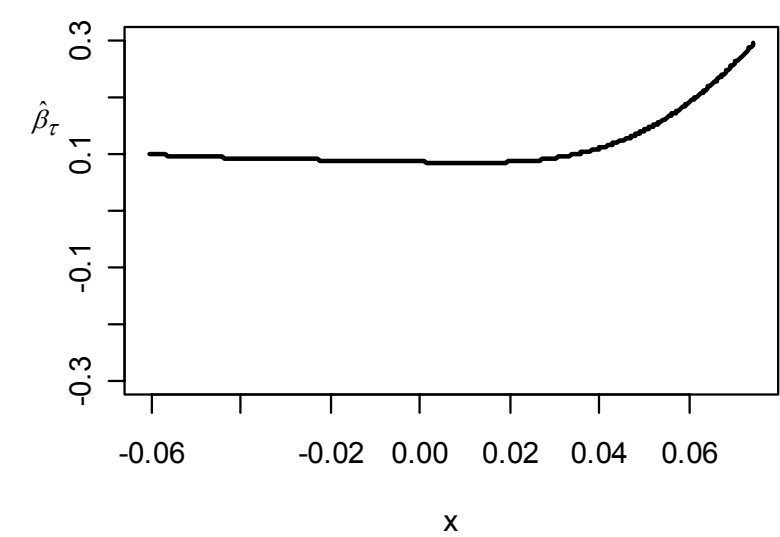

(b)

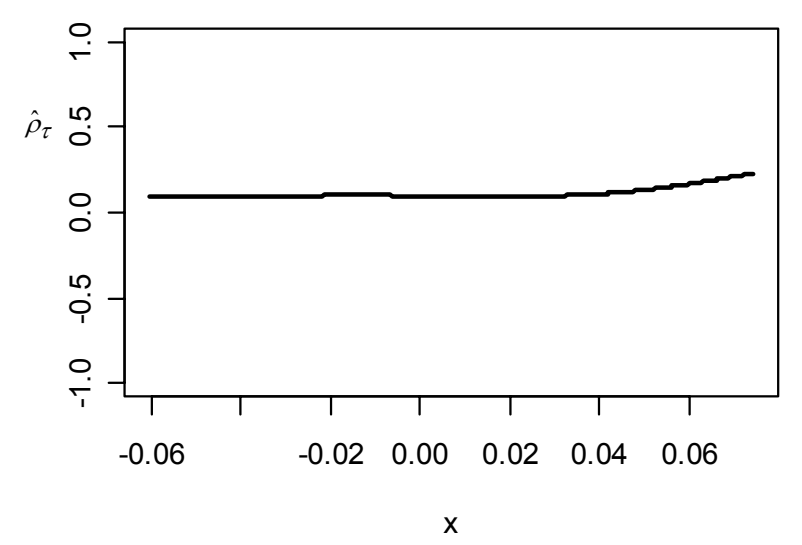

(d)

Figura 3.12 - Para os retornos do Banespa $\left(X_{\mathrm{t}}\right)$ no período de 2/jan/95 a 27/dez/00 com defasagem 1 (correlação 0,125), temos: (a) gráfico de dispersão

(b) coeficiente angular local estimado $\hat{\beta}_{\tau}(x)$, (c) variância condicional local estimada $\hat{\sigma}_{\tau}^{2}(x)$, e (d) curva de autocorrelação estimada $\hat{\rho}_{\tau}(x)$. 


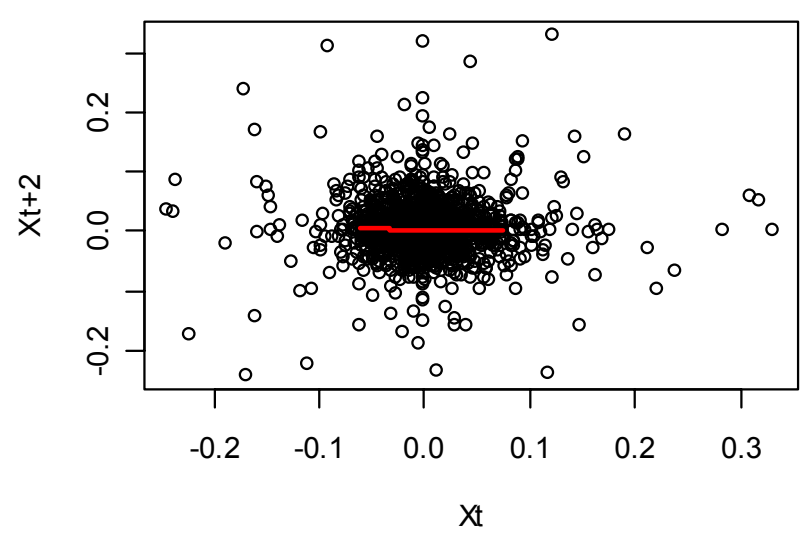

(a)

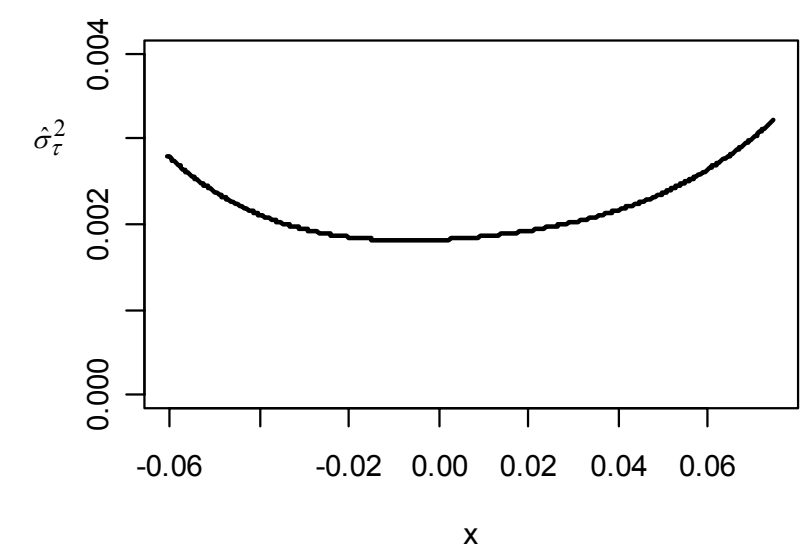

(c)

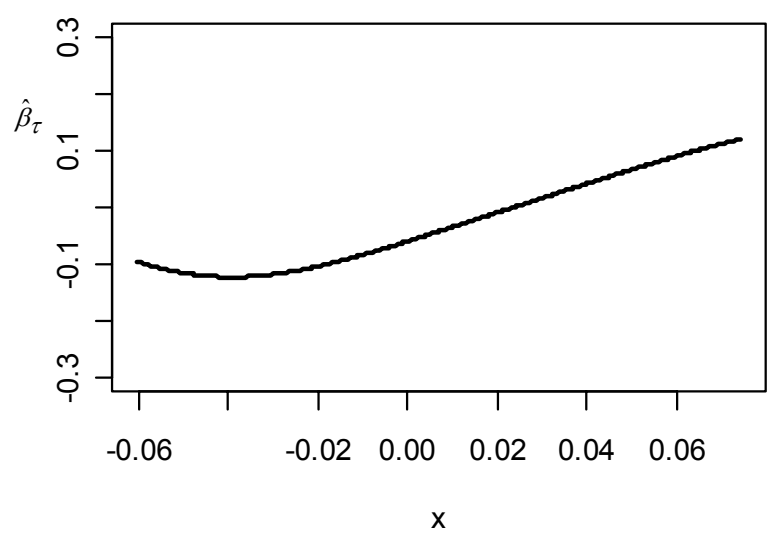

(b)

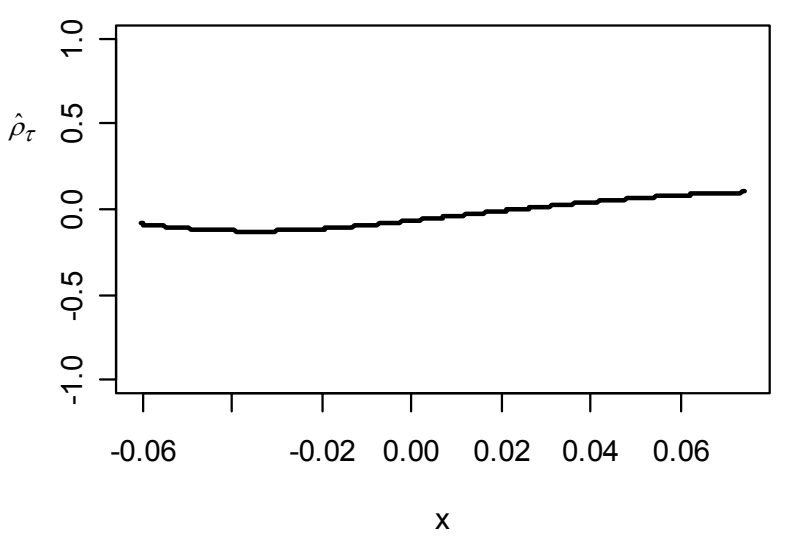

(d)

Figura 3.13 - Para os retornos do Banespa $\left(X_{\mathrm{t}}\right)$ no período de 2/jan/95 a 27/dez/00 com defasagem 2 (correlação 0,011), temos: (a) gráfico de dispersão,

(b) coeficiente angular local estimado $\hat{\beta}_{\tau}(x),(\mathrm{c})$ variância condicional local estimada $\hat{\sigma}_{\tau}^{2}(x)$, e (d) curva de autocorrelação estimada $\hat{\rho}_{\tau}(x)$. 


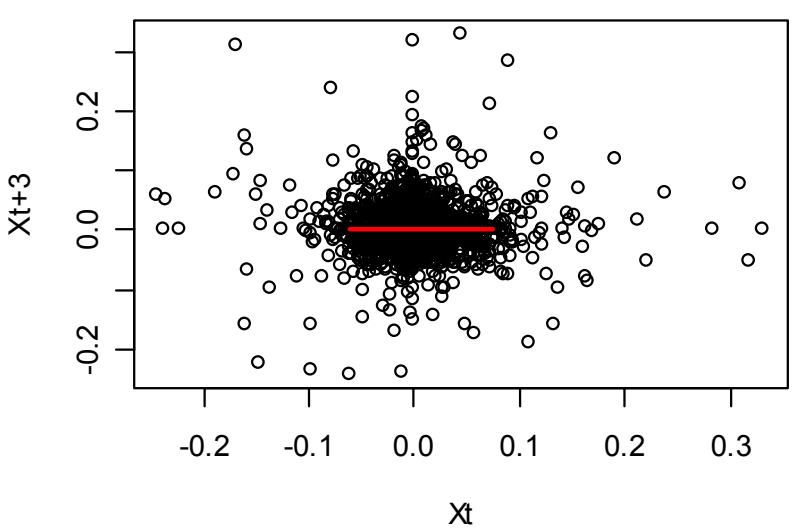

(a)

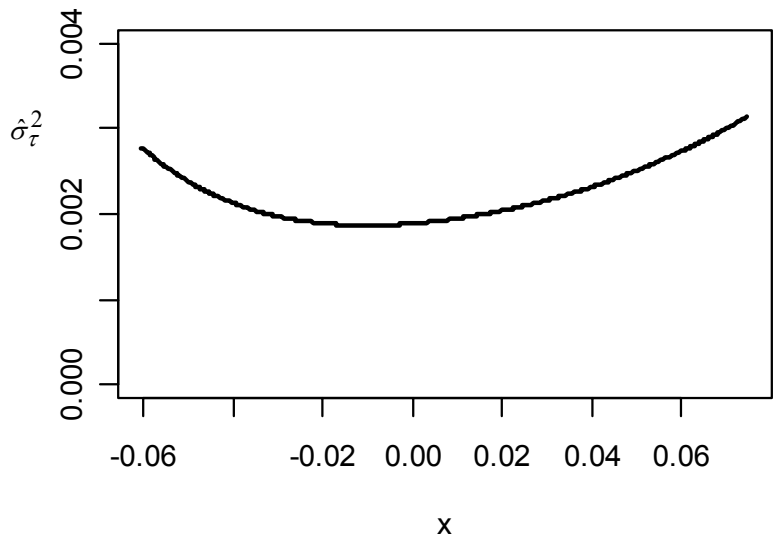

(c)

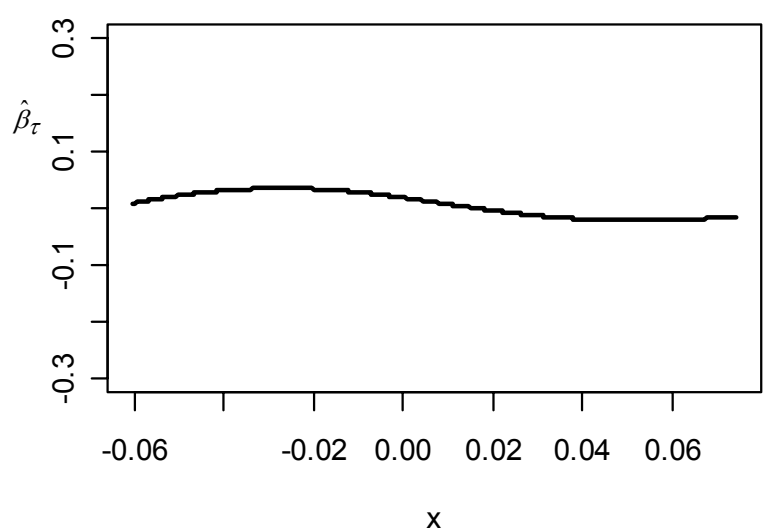

(b)

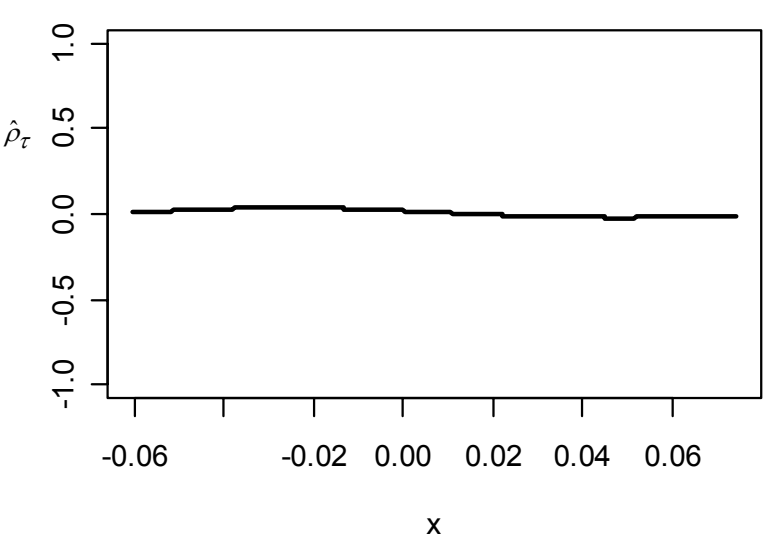

(d)

Figura 3.14 - Para os retornos do Banespa $\left(X_{\mathrm{t}}\right)$ no período de 2/jan/95 a 27/dez/00 com defasagem 3 (correlação 0,017), temos: gráfico de dispersão,

(b) coeficiente angular local estimado $\hat{\beta}_{\tau}(x)$, (c) variância condicional local estimada $\hat{\sigma}_{\tau}^{2}(x)$, e (d) curva de autocorrelação estimada $\hat{\rho}_{\tau}(x)$. 


\section{Capítulo 4}

\section{Função de Dependência de Sibuya}

\subsection{Função de Dependência Local}

Considere um vetor aleatório $(X, Y)$ contínuo. Então, a função de dependência local é dada por (1.3) ou então por (1.4).

\subsubsection{Estimadores}

A seguir, utilizando os estimadores suavizados (via funções kernel) das distribuições conjunta e marginais, propomos um estimador suavizado $\hat{\Lambda}$ para $\Lambda$ dada por (1.3), o qual é construído através do método plug-in. Além disso, derivamos um estimador empírico e outro suavizado para esta função escrita em termos de cópula, dada por (1.4).

Suponha que uma amostra aleatória $\left\{\left(X_{i}, Y_{i}\right), i=1, \ldots, n\right\}$ seja observada do vetor aleatório $(X, Y)$. Para construírmos o estimador $\hat{\Lambda}$, devemos escolher a técnica para gerar kernels multivariados e a classe de parâmetros de suavização, além do kernel univariado $\kappa$ e a largura de faixa ótima.

A forma mais geral de se definir um kernel $k$ p-dimensional (geralmente uma função densidade de probabilidade $p$-variada) é $k_{\mathbf{H}}(\mathbf{u})=|\mathbf{H}|^{-1 / 2} k\left(\mathbf{H}^{-1 / 2} \mathbf{u}\right)$ com $\int k(\mathbf{u}) d \mathbf{u}=1$ sendo $\mathbf{H}=\left\{h_{i j}^{2}\right\}$ a matriz de larguras de faixa. Existem duas técnicas usuais para gerar kernels multivariados (de ordem $p$ ) a partir de um kernel $\kappa$ univariado simétrico: o kernel simétrico radial ou esférico $k(\mathbf{u})=c_{\kappa, p} \kappa\left\{\left(\mathbf{u}^{T} \mathbf{u}\right)^{1 / 2}\right\} \quad \operatorname{com}$ $c_{\kappa, p}^{-1}=\int \kappa\left\{\left(\mathbf{u}^{T} \mathbf{u}\right)^{1 / 2}\right\} d \mathbf{u}$, e o kernel produto $k(\mathbf{u})=\prod_{i=1}^{p} \kappa\left(u_{i}\right)$. Observamos, no entanto, que o kernel produto também pode ser definido como sendo o produto de diferentes kernels (Härdle, 1991).

Wand e Jones (1995) estudaram a eficiência relativa dessas duas técnicas considerando os kernels beta (uniforme, Epanechnikov, biweight, triweight), e verificaram que existe pouca diferença de desempenho no caso dos kernels bivariados.

As classes hierárquicas de $\mathbf{H}$ são três: (i) $\mathbf{H} \in F$, com $F$ sendo a classe das matrizes simétricas positivas definidas $p \times p$, então, $\mathbf{H}$ possui $p(p+1) / 2$ entradas independentes; neste caso, a suavização pode ter diferentes direções em relação ao eixo das coordenadas; (ii) $\mathbf{H} \in \mathscr{D}, \mathscr{D} \subset \mathcal{F}$, com $\mathcal{D}$ sendo a subclasse das matrizes positivas definidas diagonais $p \times p$, portanto, $\mathbf{H}=\operatorname{diag}\left(h_{1}^{2}, \ldots, h_{p}^{2}\right)$; aqui, a suavização pode ser diferente nas $p$ direções das coordenadas; (iii) $\mathbf{H} \in S, S \subset F$, sendo a subclasse das matrizes positivas definidas e proporcionais à matriz identidade, ou seja, $\mathbf{H}=h^{2} \mathbf{I}$; neste caso, a suavização é igual em todas as coordenadas.

Se os dados apresentam distribuição normal multivariada com covariâncias não nulas (há rotação na massa de probabilidades), então é indicado o uso de $\mathbf{H} \in F$, no caso, $\mathbf{H}=h^{2} \mathbf{S}$ com $\mathbf{S}$ sendo a matriz de 
covariâncias amostrais. Caso contrário, se esta distribuição apresentar covariâncias nulas, então a eficiência das estimativas é similar se utilizarmos $\mathbf{H} \in \mathcal{F}$ ou $\mathbf{H} \in \mathcal{D}$. Por outro lado, se a distribuição dos dados não é normal multivariada, devemos tomar muito cuidado com o uso da parametrização $\mathbf{H} \in F$, pois ela pode resultar em estimativas muito ruins (Wand e Jones, 1995 - página 107).

Para $\hat{\Lambda}$ iremos considerar o kernel bivariado tipo simétrico (ou produto) e larguras de faixa $\mathbf{H}=\operatorname{diag}\left(h_{1}^{2}, h_{2}^{2}\right)$.

Então, seja $k: \Re^{2} \rightarrow \Re$ uma função kernel bivariada tal que $\iint k(u, v) d u d v=1 \quad \mathrm{e}$ $k\left(x, y ; h_{1}, h_{2}\right)=\frac{1}{h_{1} h_{2}} k\left(\frac{x}{h_{1}}, \frac{y}{h_{2}}\right)$, com $h_{j}>0, j=1,2$, sendo funções de $n$ tais que $h_{j} \rightarrow 0$ conforme $n \rightarrow \infty$. Então, o estimador da densidade conjunta $f(x, y)$ é dado por $\hat{f}(x, y)=\frac{1}{n} \sum_{i=1}^{n} k\left(x-X_{i}, y-Y_{i} ; h_{1}, h_{2}\right)$ $=\frac{1}{n h_{1} h_{2}} \sum_{i=1}^{n} k\left(\frac{x-X_{i}}{h_{1}}, \frac{y-Y_{i}}{h_{2}}\right)$. Considere $K(x, y)=\int_{-\infty}^{x} \int_{-\infty}^{y} k(u, v) d u d v$ a função primitiva de $k(x, y)$ com $K\left(x, y ; h_{1}, h_{2}\right)=K\left(\frac{x}{h_{1}}, \frac{y}{h_{2}}\right)$. Então, o estimador suavizado da função de distribuição conjunta $F(x, y)$ é dado por $\hat{F}(x, y)=\int_{-\infty}^{x} \int_{-\infty}^{y} \hat{f}(u, v) d u d v=\frac{1}{n} \sum_{i=1}^{n} K\left(x-X_{i}, y-Y_{i} ; h_{1}, h_{2}\right)=\frac{1}{n} \sum_{i=1}^{n} K\left(\frac{x-X_{i}}{h_{1}}, \frac{y-Y_{i}}{h_{2}}\right)$.

Sejam as funções kernels $k_{j}(),. j=1,2$, simétricas, limitadas e reais, tais que $\int k_{j}(u) d u=1$ e $k_{j}\left(u ; h_{j}\right)=\frac{1}{h_{j}} k_{j}\left(\frac{u}{h_{j}}\right)$. Então, os estimadores das densidades marginais $f_{1}(x)$ e $f_{2}(y)$ são dados por $\hat{f}_{1}(x)=\frac{1}{n} \sum_{i=1}^{n} k_{1}\left(x-X_{i} ; h_{1}\right)=\frac{1}{n h_{1}} \sum_{i=1}^{n} k_{1}\left(\frac{x-X_{i}}{h_{1}}\right)$ e $\hat{f}_{2}(y)=\frac{1}{n h_{2}} \sum_{i=1}^{n} k_{2}\left(\frac{y-Y_{i}}{h_{2}}\right)$. Agora, considerando as primitivas $K_{j}($.$) de k_{j}(),. j=1,2$, dadas por $K_{j}(w)=\int_{-\infty}^{w} k_{j}(u) d u \quad \operatorname{com} K_{j}\left(w ; h_{j}\right)=K_{j}\left(\frac{w}{h_{j}}\right)$, então o estimador suavizado das funções de distribuição marginais $F_{1}(x)$ e $F_{2}(y)$ são dados por $\hat{F}_{1}(x)=\int_{-\infty}^{x} \hat{f}(u) d u$ $=\frac{1}{n} \sum_{i=1}^{n} K_{1}\left(x-X_{i} ; h_{1}\right)=\frac{1}{n} \sum_{i=1}^{n} K_{1}\left(\frac{x-X_{i}}{h_{1}}\right)$ e $\hat{F}_{2}(y)=\frac{1}{n} \sum_{i=1}^{n} K_{2}\left(\frac{y-Y_{i}}{h_{2}}\right)$.

Portanto, um estimador suavizado $\hat{\Lambda}$ de $\Lambda$ dada por (1.3) é

$$
\hat{\Lambda}\left(\hat{F}_{1}(x), \hat{F}_{2}(y)\right)=\frac{\hat{F}(x, y)}{\hat{F}_{1}(x) \hat{F}_{2}(y)}, \forall(x, y) \in G, \operatorname{com} \hat{F}_{1}(x), \hat{F}_{2}(y)>0,
$$

em que $\hat{F}(.,),. \hat{F}_{1}($.$) e \hat{F}_{2}($.$) são como acima, e G$ é um conjunto de pontos bivariados de grade em $\Re^{2}$.

Agora, vejamos dois estimadores não paramétricos para $\Lambda$ dada por (1.4). 
Considerando os estimadores empíricos da distribuição conjunta e das distribuições marginais de $X$ e $Y$ dados, respectivamente, por $\quad F_{n}(x, y)=\frac{1}{n} \sum_{i=1}^{n} \mathrm{I}\left(X_{i} \leq x, Y_{i} \leq y\right), \quad F_{1 n}(x)=\frac{1}{n} \sum_{i=1}^{n} I\left(X_{i} \leq x\right) \quad$ (ou $\left.F_{1 n}=F_{n}(x,+\infty)\right)$ e $F_{2 n}(y)=\frac{1}{n} \sum_{i=1}^{n} I\left(Y_{i} \leq y\right) \quad\left(\right.$ ou $\left.F_{2 n}=F_{n}(+\infty, y)\right)$, então conforme Fermanian et al. (2004), utilizando o método plug-in temos que $C_{n}(u, v)=F_{n}\left(F_{1 n}^{-1}(u), F_{2 n}^{-1}(v)\right), \forall(u, v) \in[0,1]^{2}$, é a chamada cópula empírica. Portanto, um estimador empírico para (1.4) é dado por

$$
\Lambda_{n}(u, v)=\frac{C_{n}(u, v)}{u v}, \forall(u, v) \in G,
$$

em que $F_{1 n}^{-1}(u)=\inf \left\{x: F_{1 n}(x) \geq u\right\}, F_{2 n}^{-1}(v)=\inf \left\{y: F_{2 n}(y) \geq v\right\}$ e $G$ é um conjunto de pontos bivariados de grade em $(0,1]^{2}$.

Mas, para obtermos melhores resultados, propomos o uso de um estimador suavizado via kernel o qual utiliza o método plug-in. Considere $\hat{F}, \hat{F}_{1}$ e $\hat{F}_{2}$ dados acima. Então, conforme Fermanian e Scaillet (2003), o estimador suavizado para a cópula é dado por $\hat{C}(u, v)=\hat{F}\left(\hat{F}_{1}^{-1}(u), \hat{F}_{2}^{-1}(v)\right)$ em que $\hat{F}_{1}^{-1}(u)=\inf \left\{x: \hat{F}_{1}(x) \geq u\right\}$ e $\hat{F}_{2}^{-1}(v)=\inf \left\{y: \hat{F}_{2}(y) \geq v\right\}$. Como conseqüência, o correspondente estimador suavizado para (1.4) resulta em

$$
\hat{\Lambda}(u, v)=\frac{\hat{C}(u, v)}{u v}, \forall(u, v) \in G .
$$

Para estimar a função densidade, sabemos que o kernel ótimo é o Epanechnikov, mas que outros kernels apresentam desempenhos similares. Para estimar a função de distribuição, o kernel ótimo é o kernel uniforme (Jones, 1990), mas aqui também, o desempenho de outros kernels são similares. Portanto, a escolha do kernel pode ser de acordo com a suavidade da função ou a facilidade computacional, por exemplo.

Já a escolha do valor do parâmetro de suavização é o ponto crítico, tanto para a estimação da densidade quanto para a estimação da distribuição. Porém, a ordem do parâmetro de suavização ótimo para estimar a função densidade univariada é $n^{-1 / 5}$ (e para a $p$-variada é $n^{-1 /(p+4)}$ - Silverman, 1986), enquanto que para a função de distribuição univariada é $n^{-1 / 3}$. Por exemplo, o parâmetro de suavização ótimo para a função de distribuição univariada foi derivado por Azzalini (1981) através da minimização do erro quadrático médio. Este autor sugere $h=1,3 \sigma n^{-1 / 3}$ para a estimação nos extremos das caudas da distribuição e $h=0,5 \sigma n^{-1 / 3}$ para a estimação nas demais regiões. Já Hansen (2004), através da minimização o erro quadrático médio integrado assintótico, obteve a largura de faixa de referência $\hat{h}=1,59 \hat{o n}^{-1 / 3}$, se a distribuição dos dados é normal e se o kernel é o Gaussiano.

Observamos que, se $h=0$, então o estimador suavizado torna-se o estimador empírico.

\subsubsection{Consistência dos estimadores}

A seguir, vamos obter as propriedades dos estimadores (4.1), (4.2) e (4.3).

Consideremos primeiramente (4.1). 
Sejam as condições de regularidade a seguir. Observamos que estamos supondo a mesma largura de faixa $h$ para cada marginal por conveniência de notação, mas esta suposição pode ser facilmente removida (Fermanian et al., 2004).

(C1) $F$ tem derivada limitada de ordem $q$;

(C2) $F_{1}$ e $F_{2}$ são Lipschitzianas;

(C3) $h \rightarrow 0$, quando $n \rightarrow \infty$;

(C4) $\lim _{n \rightarrow \infty} \sqrt{n} h^{q}=0$;

(C5) $\int_{\mathfrak{R}} \int_{\mathfrak{R}} x^{k} y^{l} k(x, y) d x d y=0,1 \leq k+l<q$;

(C6) $\int_{\Re} \int_{\Re}|x|^{k}|y|^{l}|k(x, y)| d x d y<\infty, 1 \leq k+l \leq q$;

(C7) $\int_{\mathfrak{R}} \int_{\mathfrak{R}}(|x|+|y|) d K(x, y)<\infty$.

Teorema 4.1. Satisfeitas as condições (C1) a (C7) anteriores, então

$$
\hat{\Lambda}\left(\hat{F}_{1}(x), \hat{F}_{2}(y)\right) \underset{n \rightarrow \infty}{\stackrel{P}{\longrightarrow}} \Lambda\left(F_{1}(x), F_{2}(y)\right) \text {, para cada }(x, y) \in \mathfrak{R}^{2} \text { fixado, }
$$

$\operatorname{com} \hat{F}_{1}(x), \hat{F}_{2}(y)>0$ e $F_{1}(x), F_{2}(y)>0$.

prova:

Satisfeitas as condições (C1) e (C4) a (C6), então o Lema 8 de Fermanian et al. (2004) é válido, o qual resulta numa das condições do Lema 7 destes autores. Agora, considere válidas também as condições (C2), (C3) e (C7), então o mencionado Lema 7 é válido, ou seja, o processo suavizado $\{\sqrt{n}(\hat{F}(x, y)-F(x, y)), x, y \in \mathfrak{R}\}$, converge fracamente para uma ponte Browniana em $D\left(\Re^{2}\right)$, conforme $n \rightarrow \infty$, sendo $D\left(\Re^{2}\right)$ o espaço das funções em $\mathfrak{R}^{2}$ que são contínuas à direita e têm limites à esquerda. Portanto, pelo Teorema 2.3.4. de Lehmann (1999), $\hat{F}(x, y) \stackrel{P}{\longrightarrow} F(x, y)$ quando $n \rightarrow \infty$. E como $\hat{F}_{1}(x)=\hat{F}(x, \infty), \quad \hat{F}_{2}(y)=\hat{F}(\infty, y)$, $F_{1}(x)=F(x, \infty)$ e $F_{2}(y)=F(\infty, y)$, então pelo Teorema 5.1.4 de Fuller, temos que $\hat{F}_{1}(x) \stackrel{P}{\longrightarrow} F_{1}(x)$ e $\hat{F}_{2}(y) \stackrel{P}{\longrightarrow} F(y)$, conforme $n \rightarrow \infty$.

Agora, considere $\hat{\boldsymbol{\theta}}=\left(\hat{\theta}_{1}, \hat{\theta}_{2}, \hat{\theta}_{3}\right)^{\prime}=\left(\hat{F}(x, y), \hat{F}_{1}(x), \hat{F}_{2}(y)\right)^{\prime}, \quad \boldsymbol{\theta}=\left(\theta_{1}, \theta_{2}, \theta_{3}\right)^{\prime}=\left(F(x, y), F_{1}(x), F_{2}(y)\right)^{\prime} \quad$ e $g(\mathbf{w}): \mathfrak{R}^{3} \rightarrow \Re$ uma função contínua definida por $g(\mathbf{w})=w_{1} /\left(w_{2} w_{3}\right)$ com $\mathbf{w}=\left(w_{1}, w_{2}, w_{3}\right)^{\prime}$ e $w_{1}, w_{2}>0$. Vimos que $\hat{\theta}_{i} \stackrel{P}{\longrightarrow} \theta_{i}, i=1,2,3$, quando $n \rightarrow \infty$. Então, pelo Lema 5.1.3 de Fuller (1976), $\hat{\boldsymbol{\theta}} \stackrel{P}{\longrightarrow} \boldsymbol{\theta}$, e pelo Teorema 5.1.4 de Fuller, $g(\hat{\boldsymbol{\theta}}) \stackrel{P}{\longrightarrow} g(\boldsymbol{\theta})$, ou seja, $\hat{F}(x, y) /\left(\hat{F}_{1}(x) \hat{F}_{2}(y)\right) \stackrel{P}{\longrightarrow} F(x, y) /\left(F_{1}(x) F_{2}(y)\right)$. Portanto, $\hat{\Lambda}\left(\hat{F}_{1}(x), \hat{F}_{2}(y)\right) \stackrel{P}{\longrightarrow} \Lambda\left(F_{1}(x), F_{2}(y)\right)$, conforme $n \rightarrow \infty$, para todo $(x, y) \in \mathfrak{R}^{2}$ fixado.

A seguir obtemos a consistência do estimador empírico (4.2). 
Teorema 4.2. Suponha que $F$ tenha funções distribuições marginais contínuas e que a função cópula $C(u, v)$ tenha derivadas parciais contínuas. Então

$$
\Lambda_{n}(u, v) \underset{n \rightarrow \infty}{\stackrel{P}{\longrightarrow}} \Lambda(u, v), \text { para cada }(u, v) \in(0,1]^{2} \text { fixado. }
$$

prova:

Pelo Teorema 3 de Fermanian et al. (2004), satisfeitas as suposições deste teorema, então o processo da cópula empírica $\left\{Z_{n}(u, v) \equiv \sqrt{n}\left(C_{n}(u, v)-C(u, v)\right), 0 \leq u, v \leq 1\right\}$, converge fracamente para um processo Gaussiano $\left\{G_{C}(u, v), 0 \leq u, v \leq 1\right\}$ em $l^{\infty}\left([0,1]^{2}\right)$, que é o espaço das funções limitadas quase certamente em $[0,1]^{2}$ com norma do supremo. Então, pelo Teorema 2.3.4 de Lehmann (1999), se $n \rightarrow \infty$ então $C_{n}(u, v) \stackrel{P}{\longrightarrow} C(u, v)$, para todo $(u, v) \in(0,1]^{2}$ fixado. E como uma função desse estimador também converge em probabilidade, obtemos o resultado.

Finalmente, obtemos a consistência do estimador (4.3).

Teorema 4.3. Satisfeitas as condições $(\mathrm{C} 1)$ a (C7) anteriores, e supondo que $C$ tenha derivadas parciais contínuas, então

$$
\hat{\Lambda}(u, v) \underset{n \rightarrow \infty}{\stackrel{P}{\longrightarrow}} \Lambda(u, v) \text {, para cada }(u, v) \in(0,1]^{2} \text { fixado. }
$$

prova:

Satisfeitas as condições (C1) a (C7), com $C$ possuindo derivadas parciais contínuas, então o Teorema 10 de Fermanian et al. (2004) é válido, ou seja, o processo da cópula suavizada $\{\hat{Z}(u, v) \equiv \sqrt{n}(\hat{C}(u, v)-C(u, v)), 0 \leq u, v \leq 1\} \quad$ converge fracamente para um processo Gaussiano $\left\{G_{C}(u, v), 0 \leq u, v \leq 1\right\}$ em $l^{\infty}\left([0,1]^{2}\right)$. E para $n \rightarrow \infty$, então $\hat{C}(u, v) \stackrel{P}{\longrightarrow} C(u, v)$, e pelo Teorema 5.1 .4 de Fuller, $\hat{\Lambda}(u, v) \stackrel{P}{\longrightarrow} \Lambda(u, v)$, para todo $(u, v) \in(0,1]^{2}$ fixado.

\subsubsection{Simulações}

Para estudarmos o comportamento da função de dependência de Sibuya $\Lambda$, elaboramos simulações da sua medida teórica e de seu estimador suavizado, inicialmente dados por (1.3) e (4.1), respectivamente, e posteriormente através de (1.4) e (4.3). Então, calculamos o viés, o erro quadrático médio e o $p$-valor do teste de normalidade de Jarque Bera em alguns pontos da diagonal principal da grade bivariada, considerando simulações com correlação 0,70 e correlação nula para cada estimador. Em ambos os casos, 200 réplicas de amostras com diferentes tamanhos foram avaliadas. Nas simulações, utilizamos grade bivariada de $31 \times 31$ pontos, estimador $\hat{\Lambda}$ com kernel produto, $90 \%$ dos dados centrais, kernel Gaussiano e largura de faixa ótima de Hansen (2004).

Primeiramente, simulamos a medida com correlação 0,70. A função teórica (1.3) foi construída considerando um vetor aleatório $(X, Y)$ com distribuição normal de média $(3,05 ; 6,44)^{\prime}$ e $\operatorname{vec}(\boldsymbol{\Sigma})=(1,13 ; 1,49 ; 1,49 ; 3,99)^{\prime}$. A correspondente função estimada calculada através de (4.1), foi elaborada 
considerando 200 amostras de tamanho 1.000 observadas deste vetor aleatório. Na Figura 4.1, o gráfico de perspectiva e o gráfico de curvas de nível de $\Lambda$ apresentam-se, respectivamente, nos gráficos (a) e (b), os quais indicam considerável dependência positiva entre as duas variáveis uma vez que, a medida possui valores razoavelmente maiores que um. Este comportamento se repete nos gráficos (c) e (d) de $\hat{\Lambda}$. Comparando os gráficos (a) e (b) com (c) e (d), vemos que parece haver leve subestimação da medida para os valores mais baixos das variáveis. Na Tabela 4.1, para sete pontos da diagonal principal da grade bivariada, observamos que o viés e o erro quadrático médio de $\hat{\Lambda}$ diminuem com o aumento dos valores das variáveis, e que há subestimação de $\Lambda$ para baixos valores das variáveis. Também, exceto para um ponto de grade avaliado, a normalidade não foi rejeitada ao nível de significância de 5\%. Na Figura 4.2, apresentam-se os histogramas das 200 estimações de $\Lambda$, calculadas em alguns pontos da grade que, de modo geral, não indicam afastamento da normalidade.

Estas simulações também foram elaboradas para amostras de tamanhos 50, 100, 200 e 500, cujos resultados apresentam-se na Figura D.1 do Apêndice D. Comparando-se estas quatro simulações e a simulação analisada anteriormente $(n=1.000)$, verificamos que o viés, o erro quadrático médio e a rejeição da normalidade diminuem com o aumento do tamanho da amostra.

Em segundo lugar, a medida $\Lambda$ dada por (1.3) foi simulada com correlação nula, considerando um vetor aleatório $(X, Y)$ com distribuição normal de média $(1,33 ; 4)^{\prime}$ e $\operatorname{vec}(\boldsymbol{\Sigma})=(0,80 ; 0 ; 0 ; 2,86)^{\prime}$, e a medida estimada via (4.1) foi calculada utilizando-se 200 amostras aleatórias de tamanho 1.000 observadas deste vetor. Na Figura 4.3, observamos $\Lambda$ teórica e estimada. Comparando os gráficos (a) e (c) vemos que há uma leve subestimação da medida numa região de valores baixos para as variáveis $X$ e $Y$. Na Tabela 4.2, podemos observar este comportamento numericamente. Além disso, observamos que o viés e o erro quadrático médio diminuem com o aumento dos valores das duas variáveis aleatórias, e que o teste de normalidade de Jarque Bera não é rejeitado, exceto num ponto extremo da grade. Os histogramas dessas estimativas de $\Lambda$ podem ser verificados na Figura 4.4.

Comparando esta simulação com as simulações $n=50, n=100, n=200$ e $n=500$ (Figura D.2 do Apêndice D), verificamos que aumentando-se o tamanho da amostra, o viés geralmente diminui, o erro quadrático médio diminui, e a rejeição de normalidade diminui a partir de $n=1.000$.

Agora, comparando as simulações de correlação 0,70 com as de correlação nula, observamos que o viés e o erro quadrático médio diminuem com a redução da correlação. Já a rejeição de normalidade apresentou a seguinte inversão de comportamento: para correlação 0,70 , ocorreu maior rejeição de normalidade para $n=50$ e menor rejeição de normalidade para $n=200$ e $n=500$.

A partir de agora, vamos avaliar a função de dependência de Sibuya estimada através de (4.3), comparando-a com a respectiva função teórica dada por (1.4).

Para o vetor aleatório $(X, Y) \sim N_{2}(\boldsymbol{\mu}, \boldsymbol{\Sigma})$ com correlação 0,70 mencionado nesta seção, calculamos $\Lambda$, cuja representação gráfica encontra-se em (a) e (b) da Figura 4.5. Já $\hat{\Lambda}$ obtida através de 200 amostras de tamanho 500, apresenta-se nos gráficos (c) e (d) desta figura. Vemos que a função teórica e a estimada são muito próximas, embora haja leve subestimação da medida para baixos valores das duas variáveis, o que pode 
ser verificado também na Tabela 4.3. Ainda nesta tabela, observamos a rejeição marginal $(\alpha=0,05)$ de normalidade do estimador em somente um ponto de grade. Os respectivos histogramas apresentam-se na Figura 4.6.

Comparando esta simulação com outras de tamanhos de amostras 50, 100 e 200 (Figura D.3 do Apêndice D), vemos que com o aumento de $n$, o viés diminui, o erro quadrático médio geralmente diminui, e a rejeição de normalidade apresenta oscilações.

Comparando este estimador (4.3) com o anterior (4.1) para iguais tamanhos de amostras, vemos que este tende a apresentar menor viés nos primeiros pontos de grade, menor erro quadrático médio, e menor frequência de rejeição de normalidade para as amostras de tamanho 50 e 100.

Avaliamos também o comportamento da função para o vetor aleatório $(X, Y) \sim N_{2}(\boldsymbol{\mu}, \boldsymbol{\Sigma})$ com correlação nula analisado anteriormente. Os gráficos da função teórica e estimada apresentam-se na Figura 4.7, as quais mostram-se muito similares. Na Tabela 4.4, observamos que o erro quadrático médio é destacadamente maior no primeiro ponto de grade. Nesta tabela também vemos a não rejeição de normalidade, exceto nos dois pontos extremos da grade. Os respectivos histogramas apresentam-se na Figura 4.8.

Comparando esta simulação com outras elaboradas com diferentes tamanhos de amostras (50, 100 e 200 - veja Figura D.4 do Apêndice D), vemos que aumentando $n$, o viés geralmente diminui, o erro quadrático médio diminui, e a rejeição de normalidade oscila.

Considerando iguais tamanhos de amostras, comparamos este estimador com o anterior, e verificamos que este apresenta maior viés no primeiro ponto de grade para amostras até $n=200$, erro quadrático médio maior e menor (ou igual) rejeição de normalidade.

Agora, comparando o comportamento do estimador (4.3) para as simulações com correlação 0,70 e nula, vemos que esta última apresenta viés e erro quadrático médio menores, porém, maior rejeição de normalidade. 


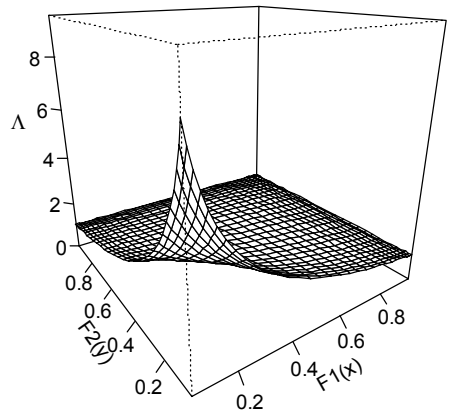

(a)

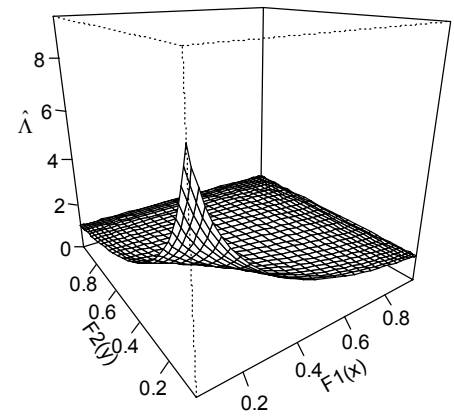

(c)

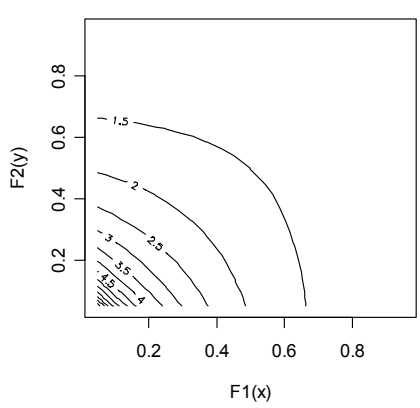

(b)

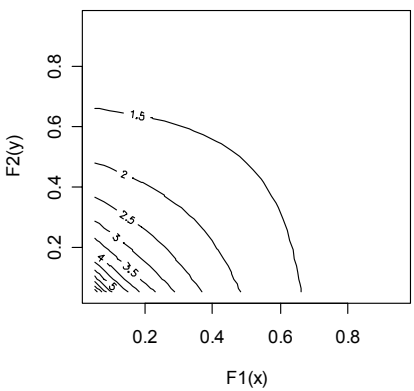

(d)

Figura 4.1 - (a) Função de dependência de Sibuya teórica via funções de distribuição, e respectivo (b) gráfico de curvas de nível , considerando um vetor aleatório $(X, Y)$ com distribuição normal de média $(3,05 ; 6,44)^{\prime}$ e vec $(\Sigma)=$ $(1,13 ; 1,49 ; 1,49 ; 3,99)^{\prime}$ (correlação 0,70). A seguir, apresenta-se a (c) função de dependência de Sibuya estimada e seu correspondente (d) gráfico de curvas de nível, utilizando 200 amostras aleatórias de tamanho 1.000.

Tabela 4.1 - Viés, erro quadrático médio e $p$-valor do teste de normalidade de Jarque Bera, para sete pontos de grade da diagonal principal da função de dependência de Sibuya estimada, considerando 200 amostras aleatórias de tamanho 1.000 , observadas de um vetor aleatório $(X, Y)$ com distribuição normal de média $(3,05 ; 6,44)^{\prime}$ e vec $(\Sigma)=(1,13 ; 1,49 ; 1,49 ; 3,99)^{\prime}$ (correlação 0,70$)$.

\begin{tabular}{|l|r|r|r|r|r|r|r|}
\hline \multicolumn{1}{|c|}{ Grade } & $\mathbf{0 , 0 5}$ & $\mathbf{0 , 1 4}$ & $\mathbf{0 , 2 9}$ & \multicolumn{1}{c|}{$\mathbf{0 , 5 0}$} & $\mathbf{0 , 7 1}$ & $\mathbf{0 , 8 6}$ & \multicolumn{1}{c|}{$\mathbf{0 , 9 5}$} \\
\hline Viés & $-0,538$ & $-0,178$ & $-0,047$ & $-0,016$ & $-0,005$ & $-0,002$ & $-0,001$ \\
\hline EQM & 1,172 & 0,076 & 0,007 & 0,001 & 0,000 & 0,000 & 0,000 \\
\hline $\boldsymbol{P}$-valor & 0,021 & 0,170 & 0,224 & 0,090 & 0,179 & 0,707 & 0,109 \\
\hline
\end{tabular}


0.05

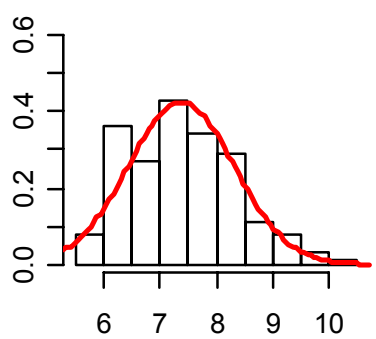

0.71

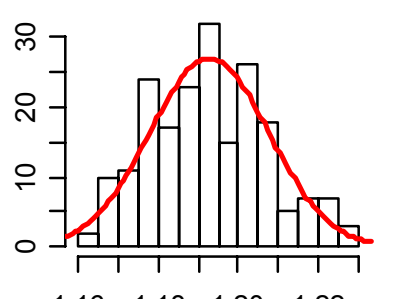

0.14

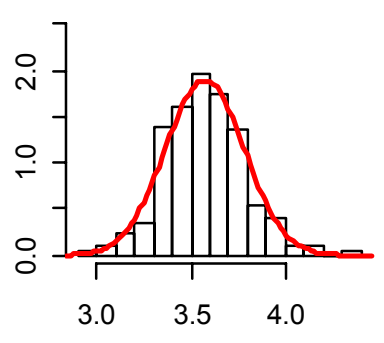

0.86

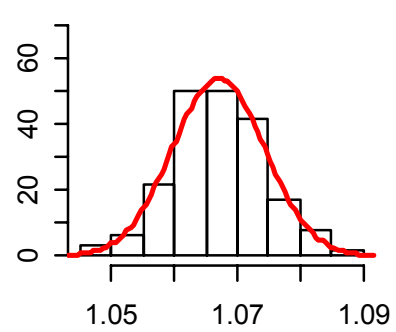

0.29

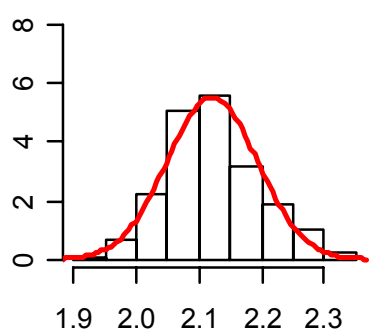

0.95

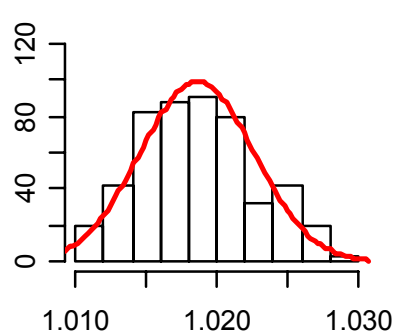

0.5

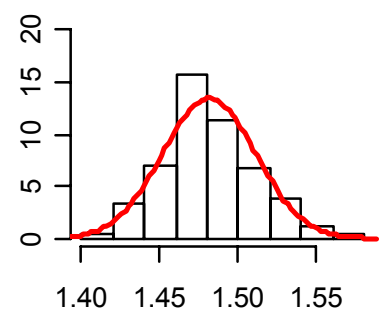

Figura 4.2 - Histogramas em pontos de grade da diagonal principal da função de dependência de Sibuya estimada através de 200 amostras de tamanho 1.000, observadas de um vetor aleatório $(X, Y)$ com distribuição

$$
\text { normal de média } \left.(3,05 ; 6,44)^{\prime} \text { e vec }(\Sigma)=(1,13 ; 1,49 ; 1,49 ; 3,99)^{\prime} \text { (correlação } 0,70\right) \text {. }
$$




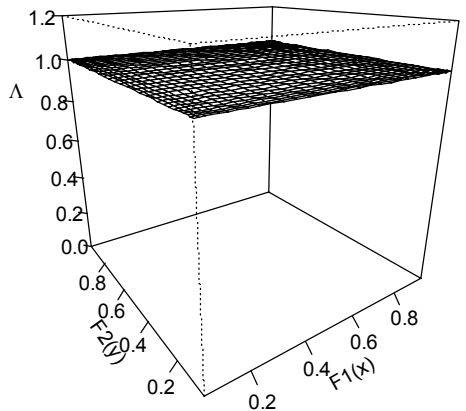

(a)

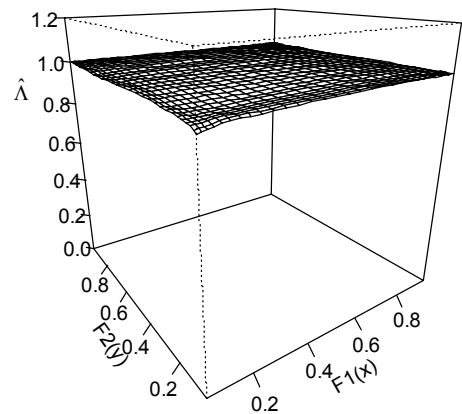

(c)

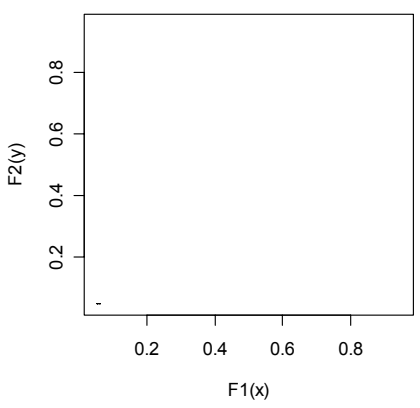

(b)

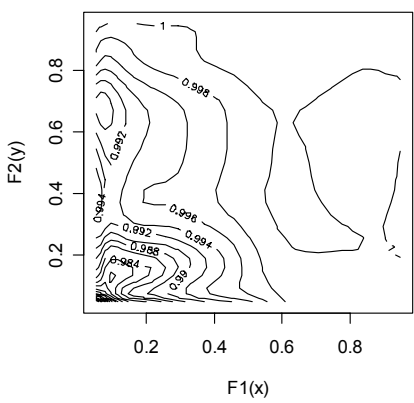

(d)

Figura 4.3 - (a) Função de dependência de Sibuya teórica via funções de distribuição e respectivo (b) gráfico de curvas de nível, considerando um vetor aleatório $(X, Y)$ com distribuição normal com média $(1,33 ; 4)^{\prime}$ e vec $(\Sigma)=$ $(0,80 ; 0 ; 0 ; 2,86)^{\prime}$ (correlação 0,0$)$. A seguir, apresenta-se a (c) função de dependência de Sibuya estimada e seu correspondente (d) gráfico de curvas de nível, utilizando 200 amostras aleatórias de tamanho 1.000.

Tabela 4.2 - Viés, erro quadrático médio e $p$-valor do teste de normalidade de Jarque Bera, para sete pontos de grade da diagonal principal da função de dependência de Sibuya estimada, considerando 200 amostras aleatórias de tamanho 1.000, observadas de um vetor aleatório $(X, Y)$ com distribuição normal de média $(1,33 ; 4)^{\prime}$ e vec $(\Sigma)=(0,80 ; 0 ; 0 ; 2,86)^{\prime}$ (correlação 0,0$)$.

\begin{tabular}{|l|c|c|c|c|c|c|c|}
\hline \multicolumn{1}{|c|}{ Grade } & $\mathbf{0 , 0 5}$ & $\mathbf{0 , 1 4}$ & $\mathbf{0 , 2 9}$ & $\mathbf{0 , 5 0}$ & $\mathbf{0 , 7 1}$ & $\mathbf{0 , 8 6}$ & $\mathbf{0 , 9 5}$ \\
\hline Viés & $-0,040$ & $-0,019$ & $-0,006$ & $-0,001$ & 0,000 & 0,000 & 0,000 \\
\hline EQM & 0,185 & 0,025 & 0,004 & 0,001 & 0,000 & 0,000 & 0,000 \\
\hline $\boldsymbol{P}$-valor & 0,503 & 0,925 & 0,723 & 0,883 & 0,959 & 0,757 & 0,025 \\
\hline
\end{tabular}


0.05

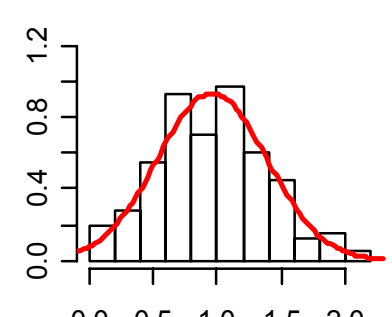

$\begin{array}{lllll}0.0 & 0.5 & 1.0 & 1.5 & 2.0\end{array}$
0.14

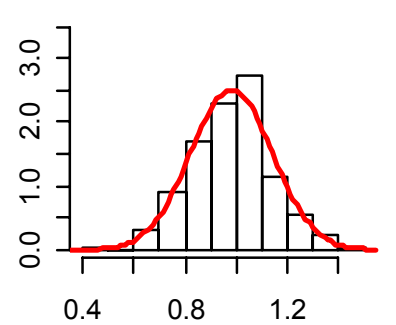

0.86

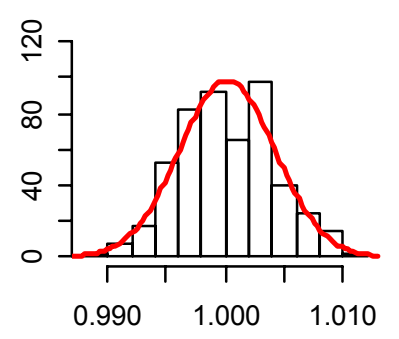

0.29

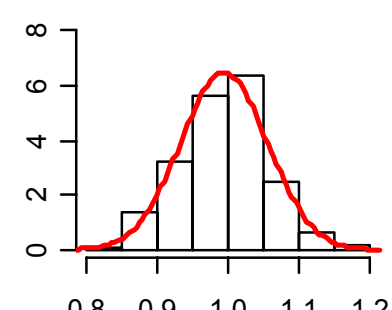

$\begin{array}{lllll}0.8 & 0.9 & 1.0 & 1.1 & 1.2\end{array}$

0.95

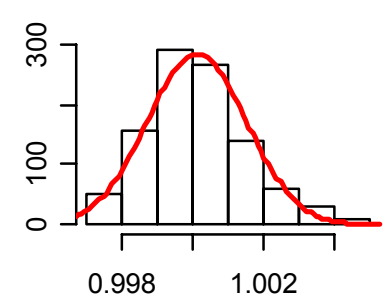

Figura 4.4 - Histogramas em pontos de grade da diagonal principal da função de dependência de Sibuya estimada através de 200 amostras de tamanho 1.000 , observadas de um vetor aleatório $(X, Y)$ com distribuição

normal de média $(1,33 ; 4)^{\prime}$ e vec $(\Sigma)=(0,80 ; 0 ; 0 ; 2,86)^{\prime}$ (correlação 0,0$)$.

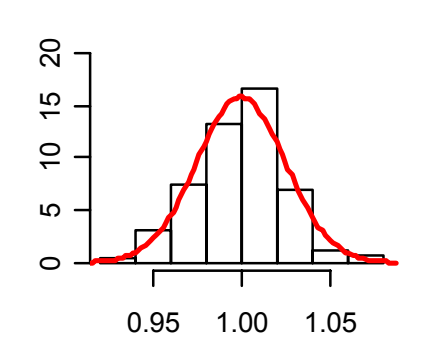

$\begin{array}{lll}0.95 & 1.00 & 1.05\end{array}$

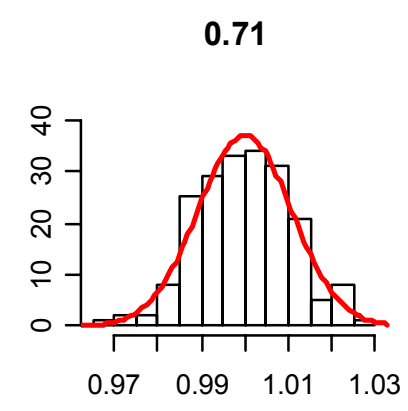




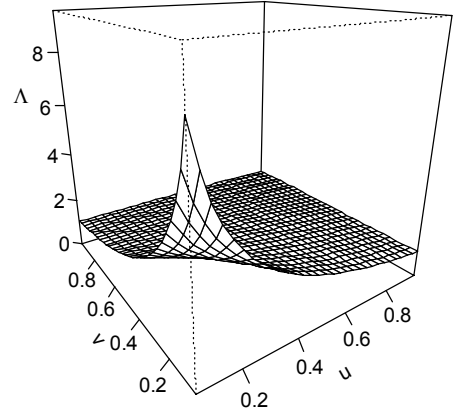

(a)

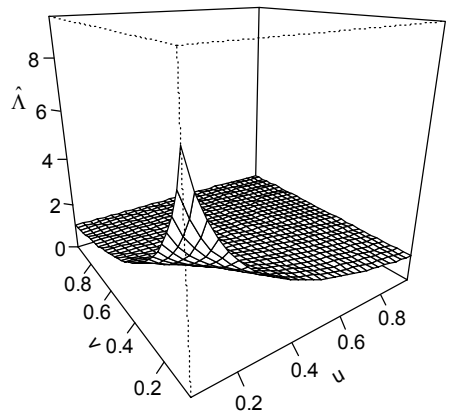

(c)

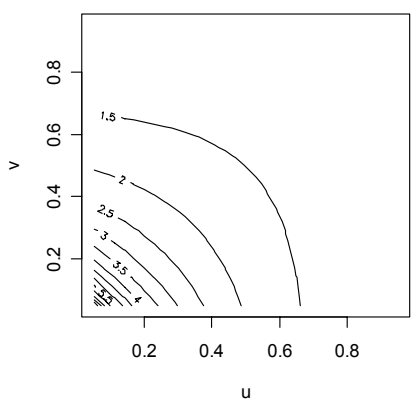

(b)

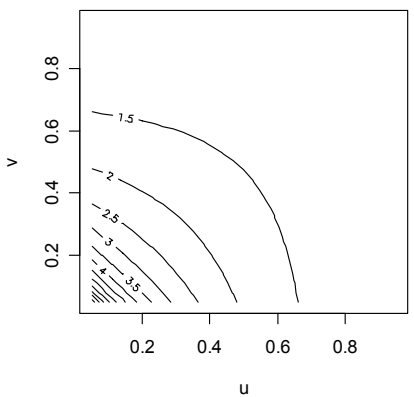

(d)

Figura 4.5 - (a) Função de dependência de Sibuya teórica via cópula e respectivo (b) gráfico de curvas de nível , considerando um vetor aleatório $(X, Y)$ com distribuição normal de média $(3,05 ; 6,44)^{\prime}$ e vec $(\Sigma)=(1,13 ; 1,49 ; 1,49 ; 3,99)^{\prime}$ (correlação 0,70). A seguir, apresenta-se a (c) função de dependência de Sibuya estimada e seu correspondente (d) gráfico de curvas de nível, utilizando 200 amostras aleatórias de tamanho 500.

Tabela 4.3 - Viés, erro quadrático médio e $p$-valor do teste de normalidade de Jarque Bera, para sete pontos de grade da diagonal principal da função de dependência de Sibuya estimada, considerando 200 amostras aleatórias de tamanho 500 , observadas de um vetor aleatório $(X, Y)$ com distribuição normal de média $(3,05 ; 6,44)^{\prime}$ e vec $(\Sigma)=(1,13 ; 1,49 ; 1,49 ; 3,99)^{\prime}$ (correlação 0,70$)$.

\begin{tabular}{|l|c|c|c|c|c|c|c|}
\hline Grade & $\mathbf{0 , 0 5}$ & $\mathbf{0 , 2 0}$ & $\mathbf{0 , 3 5}$ & $\mathbf{0 , 5 0}$ & $\mathbf{0 , 6 5}$ & $\mathbf{0 , 8 0}$ & \multicolumn{1}{c|}{$\mathbf{0 , 9 5}$} \\
\hline Viés & $-0,602$ & $-0,113$ & $-0,046$ & $-0,023$ & $-0,013$ & $-0,005$ & $-0,001$ \\
\hline EQM & 2,339 & 0,029 & 0,006 & 0,001 & 0,000 & 0,000 & 0,000 \\
\hline $\boldsymbol{P}$-valor & 0,649 & 0,478 & 0,049 & 0,620 & 0,163 & 0,345 & 0,194 \\
\hline
\end{tabular}


0.05

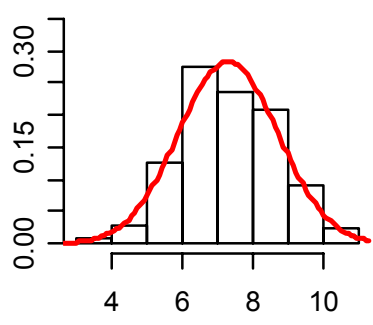

0.65

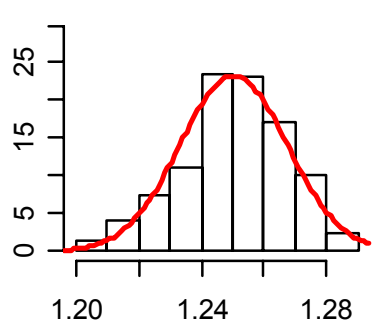

0.2
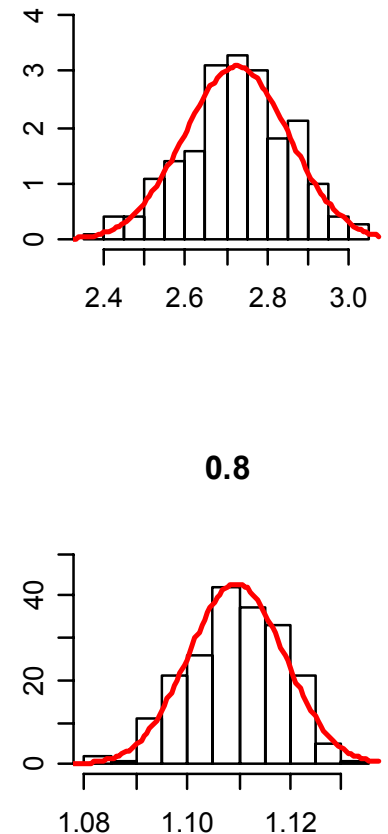

0.35

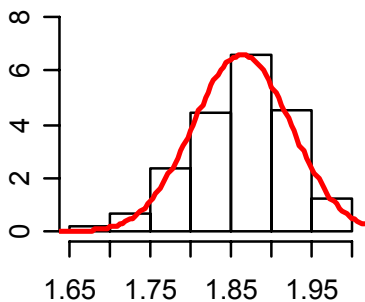

$\begin{array}{llll}1.65 & 1.75 & 1.85 & 1.95\end{array}$

0.95

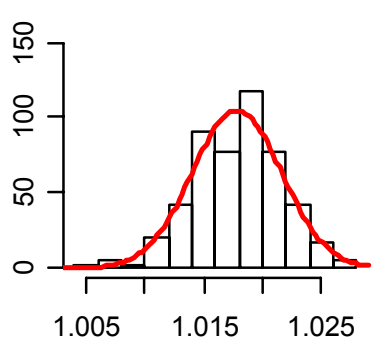

Figura 4.6 - Histogramas em pontos de grade da diagonal principal da função de dependência de Sibuya estimada através de 200 amostras de tamanho 500 , observadas de um vetor aleatório $(X, Y)$ com distribuição normal de média $(3,05 ; 6,44)^{\prime}$ e vec $(\Sigma)=(1,13 ; 1,49 ; 1,49 ; 3,99)^{\prime}$ (correlação 0,70$)$. 


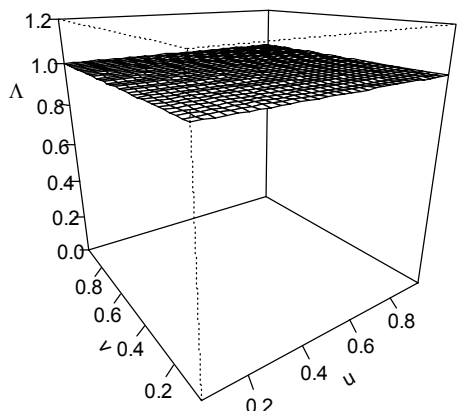

(a)

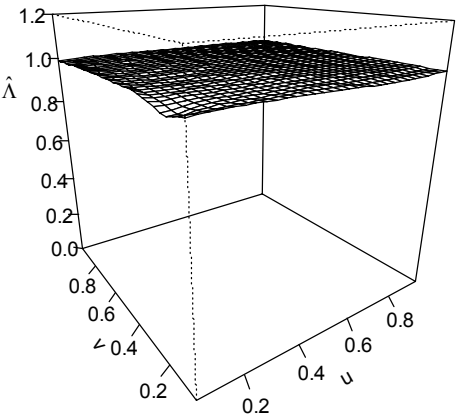

(c)

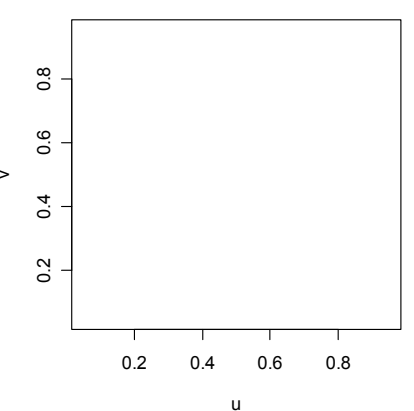

(b)

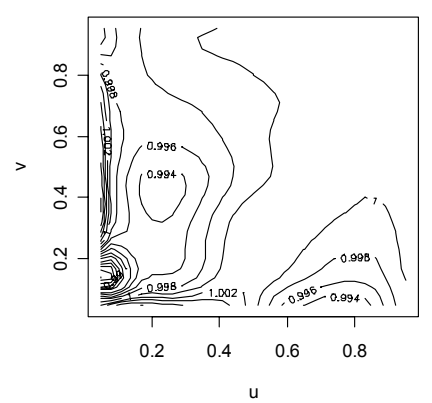

(d)

Figura 4.7 - (a) Função de dependência de Sibuya teórica via cópula e respectivo (b) gráfico de curvas de nível , considerando um vetor aleatório $(X, Y)$ com distribuição normal com média $(1,33 ; 4)^{\prime}$ e vec $(\Sigma)=(0,80 ; 0 ; 0 ; 2,86)^{\prime}$

(correlação 0,0). A seguir, apresenta-se a (c) função de dependência de Sibuya estimada e seu correspondente (d) gráfico de curvas de nível, utilizando 200 amostras aleatórias de tamanho 500.

Tabela 4.4 - Viés, erro quadrático médio e $p$-valor do teste de normalidade de Jarque Bera, para sete pontos de grade da diagonal principal da função de dependência de Sibuya estimada, considerando 200 amostras aleatórias de tamanho 500, observadas de um vetor aleatório $(X, Y)$ com distribuição normal de média $(1,33 ; 4)^{\prime}$ e $\operatorname{vec}(\Sigma)=(0,80 ; 0 ; 0 ; 2,86)^{\prime}$ (correlação 0,0$)$.

\begin{tabular}{|l|c|c|c|c|c|c|c|}
\hline Grade & $\mathbf{0 , 0 5}$ & $\mathbf{0 , 2 0}$ & $\mathbf{0 , 3 5}$ & $\mathbf{0 , 5 0}$ & $\mathbf{0 , 6 5}$ & $\mathbf{0 , 8 0}$ & $\mathbf{0 , 9 5}$ \\
\hline Viés & 0,002 & $-0,005$ & $-0,003$ & $-0,001$ & 0,001 & 0,000 & 0,000 \\
\hline EQM & 0,353 & 0,020 & 0,005 & 0,001 & 0,000 & 0,000 & 0,000 \\
\hline $\boldsymbol{P}$-valor & 0,001 & 0,767 & 0,821 & 0,831 & 0,188 & 0,915 & 0,000 \\
\hline
\end{tabular}


0.05

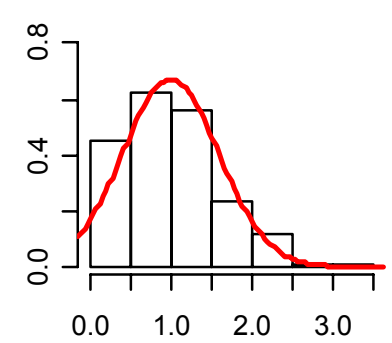

0.65

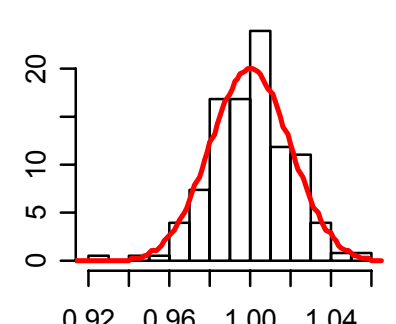

0.2

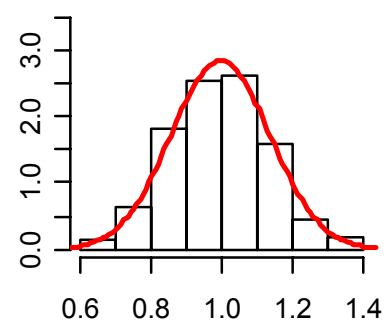

0.8

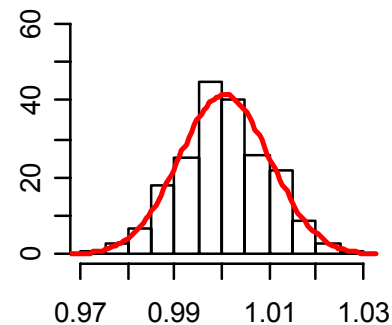

0.35

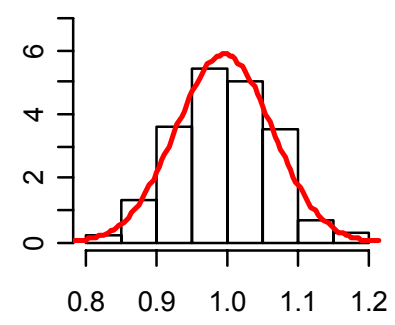

0.95

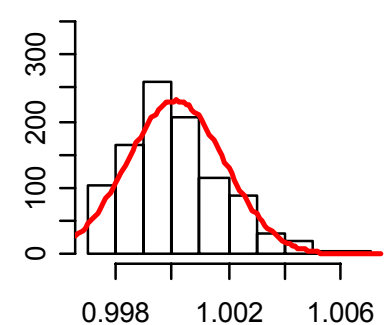

0.5

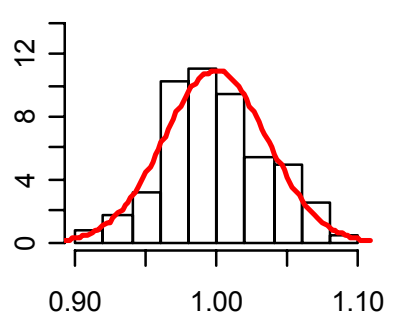

Figura 4.8 - Histogramas em pontos de grade da diagonal principal da função de dependência de Sibuya estimada através de 200 amostras de tamanho 500, observadas de um vetor aleatório $(X, Y)$ com distribuição normal de média $(1,33 ; 4)^{\prime}$ e vec $(\Sigma)=(0,80 ; 0 ; 0 ; 2,86)^{\prime}$ (correlação 0,0$)$. 


\subsubsection{Aplicações a séries reais}

A seguir, encontra-se a estimação da função de dependência de Sibuya para o preço e o volume (em milhares) das ações do S\&P500 (n=500) e também da BOVESPA $(n=63)$ em 05/out/2007. Inicialmente utilizamos o estimador (4.1) e depois o (4.3). Os parâmetros utilizados nesta estimação foram iguais às simulações anteriores, porém, agora consideramos $90 \%$ dos dados centrais.

Inicialmente, vamos analisar o preço $(X)$ e o volume (em milhares) $(Y)$ do S\&P500. A correlação linear de Pearson, o tau de Kendall e o rho de Sperman verificados entre essas variáveis foram -0,13 , -0,20 e -0,30, respectivamente. Na Figura 4.9, observamos em (a) o gráfico de dispersão dos dados, em (b) o gráfico da função de dependência de Sibuya estimada através de (4.1), e em (c) suas respectivas curvas de nível, sendo que estes dois últimos gráficos indicam que as variáveis são contramonotônicas, pois a medida apresenta valores inferiores a um. O gráfico (d) de dispersão dos postos normalizados indica leve dependência negativa entre as variáveis, e os gráficos (e) e (f) da densidade cópula empírica suavizada e de suas curvas de nível, indicam leve dependência positiva entre valores altos e valores baixos dos postos normalizados, e vice-versa, e dependência negativa entre valores baixos de ambas as variáveis. Os gráficos $(\mathrm{g})$ e (h) da cópula empírica suavizada e de suas respectivas curvas de nível, não indicam clara dependência.

Considerando agora a BOVESPA, as associações entre o preço e o volume foram de $-0,17$ (correlação de Pearson), -0,30 (tau de Kendall) e -0,44 (rho de Spearman), novamente indicando relação negativa. Na Figura 4.10, vemos o gráfico de dispersão dos dados originais em (a) e dos postos normalizados em (d), sendo que neste observamos a indicação de relação negativa entre as variáveis. Nos gráficos (b) e (c) referentes a $\hat{\Lambda}$ calculado via (4.1), observamos que as variáveis são contramonotônicas. Já nos gráficos (e) e (f) da densidade cópula empírica suavizada, observamos dependência negativa entre baixos valores de ambas as variáveis e também entre altos valores, dependência positiva entre baixos valores de uma variável e altos valores da outra variável, e independência entre valores intermediários de ambas as variáveis. Nos gráficos $(\mathrm{g})$ e (h), vemos a indicação de dependência negativa.

Agora, utilizando o estimador via cópulas dado por (4.3), observamos novamente a dependência negativa para os dados do S\&P500 (Figura 4.11) e da BOVESPA (Figura 4.12). 


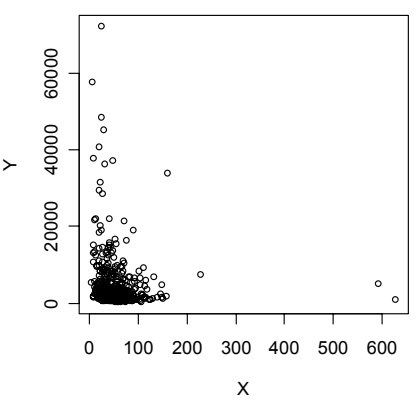

(a)

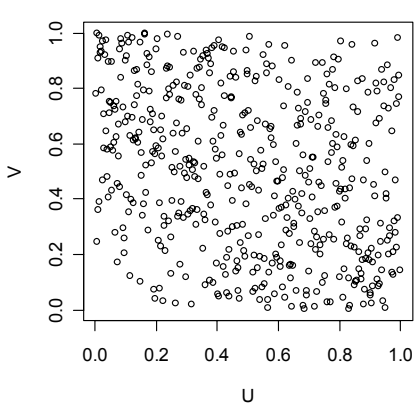

(d)

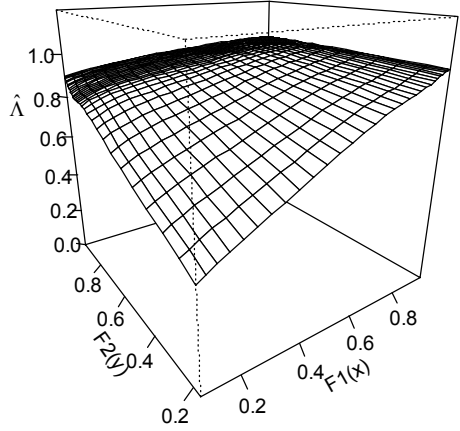

(b)

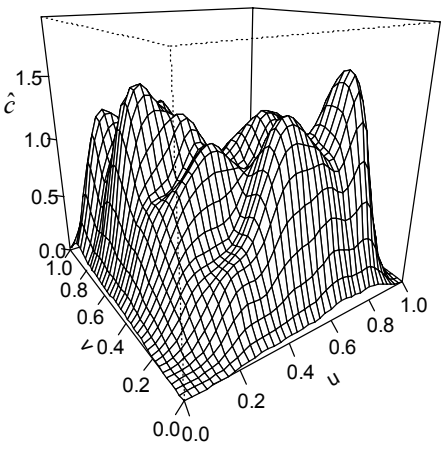

(e)

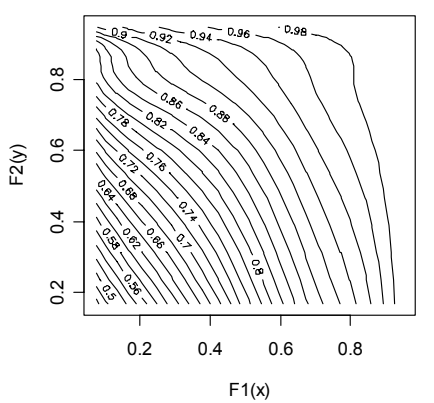

(c)

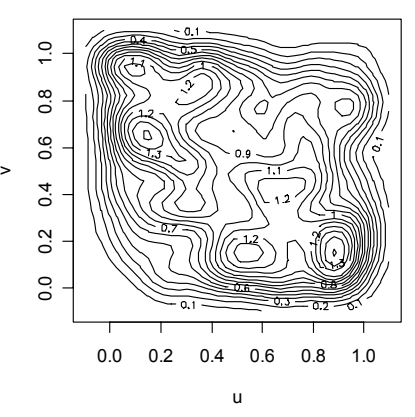

(f)

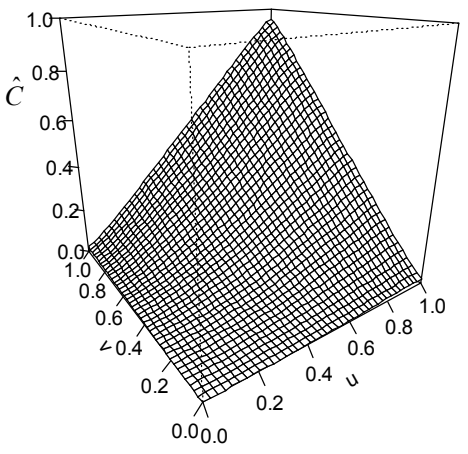

(g)

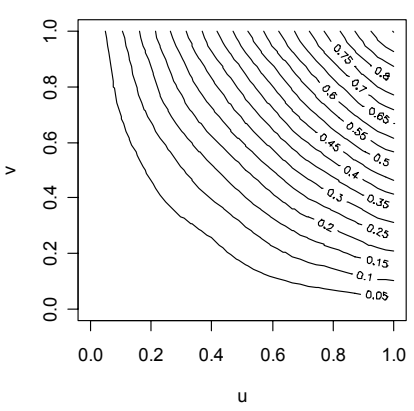

(h)

Figura 4.9 - Considerando o Preço $(X)$ e Volume (em milhares) $(Y)$ do S\&P500 em 05/out/2007, temos: (a) gráfico de dispersão, (b) função de dependência de Sibuya estimada via funções de distribuição e respectivo (c) gráfico de curvas de nível. Para os correspondentes postos normalizados, temos o (d) gráfico de dispersão, (e) densidade cópula suavizada e suas (f) curvas de nível. Em (g), a cópula suavizada e suas (h) curvas de nível. 


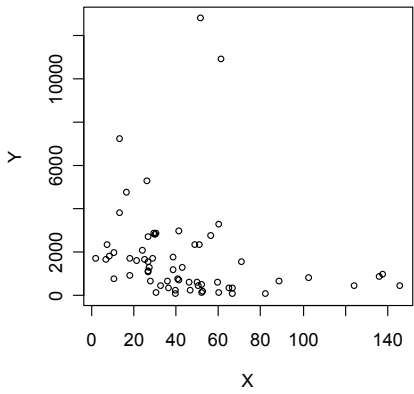

(a)

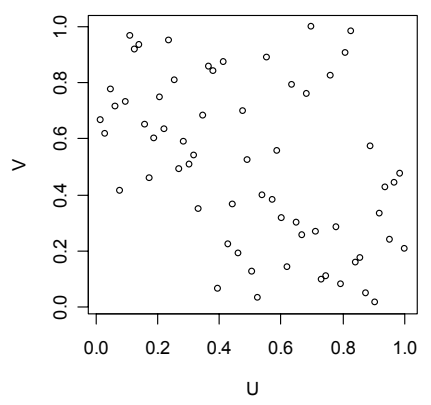

(d)

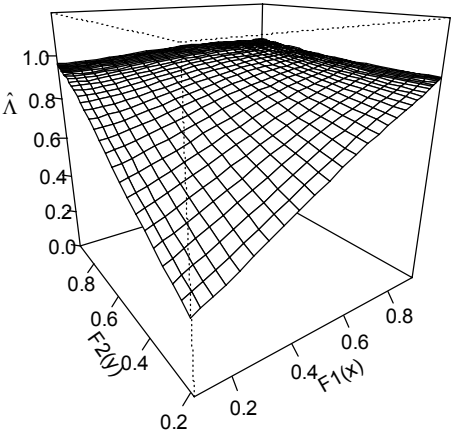

(b)

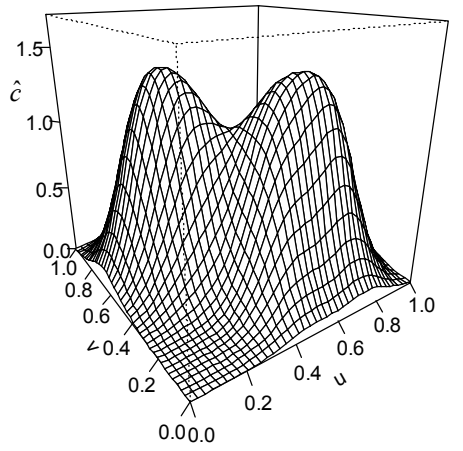

(e)

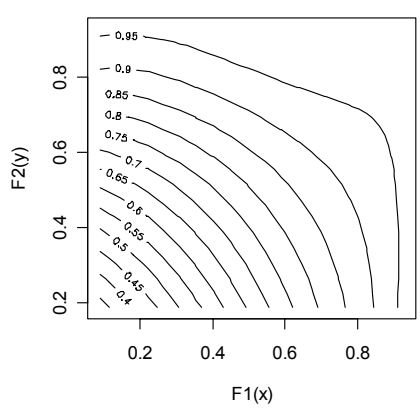

(c)

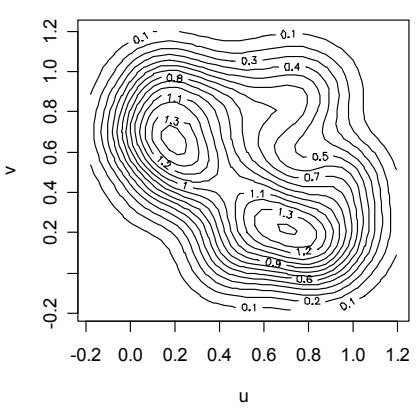

(f)

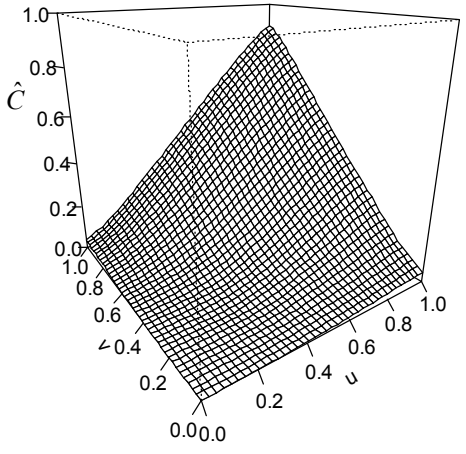

(g)

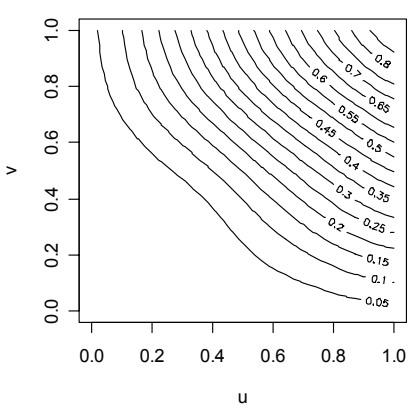

(h)

Figura 4.10 - Considerando o Preço $(X)$ e Volume (em milhares) $(Y)$ da BOVESPA em 05/out/2007, temos: (a) gráfico de dispersão, (b) função de dependência de Sibuya estimada via funções de distribuição e respectivo (c) gráfico de curvas de nível. Para os correspondentes postos normalizados, temos o (d) gráfico de dispersão, (e) densidade cópula suavizada e suas (f) curvas de nível. Em (g), a cópula suavizada e suas (h) curvas de nível. 


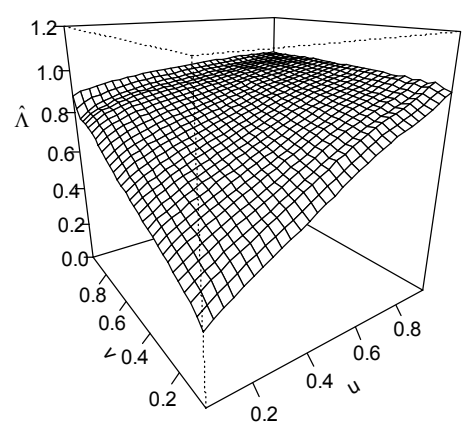

(a)

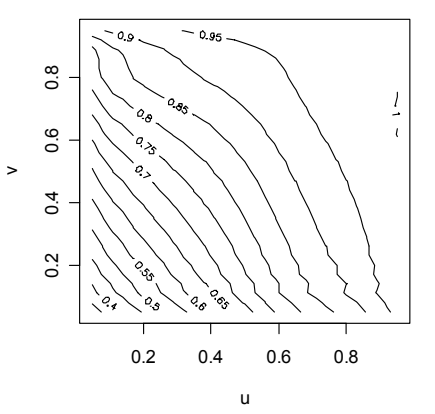

(b)

Figura 4.11 - Considerando o Preço $(X)$ e o Volume (em milhares) ( $Y$ ) do S\&P500 em 05/out/2007, temos a função de dependência de Sibuya estimada via cópula em (a) e respectivo gráfico de curvas de nível em (b).

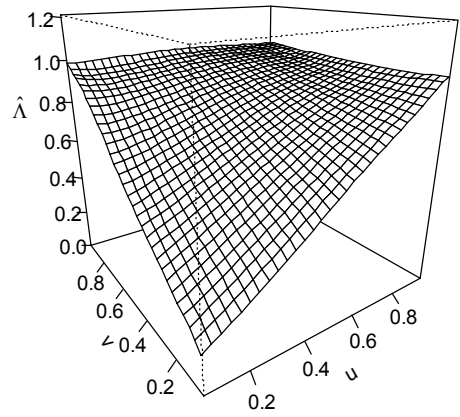

(a)

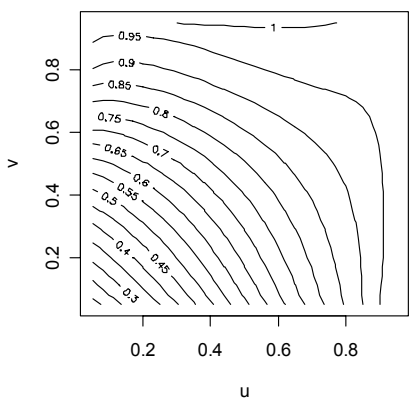

(b)

Figura 4.12 - Considerando o Preço $(X)$ e Volume (em milhares) $(Y)$ da BOVESPA em 05/out/2007, temos a função de dependência de Sibuya estimada em (a) e respectivo gráfico de curvas de nível em (b). 


\subsection{Função de Dependência Cruzada Contemporânea Local}

Considere $\left\{\left(X_{t}, Y_{t}\right), t \in Z\right\}$ um processo estacionário estrito a valores contínuos. Seja $F(x, y)$ a distribuição conjunta de $X_{t}$ e $Y_{t}, F_{1}(x)$ e $F_{2}(y)$ as respectivas distribuições marginais, e $C(u, v)$ a cópula correspondente, $\forall t \in Z$.

Então, a função de dependência de Sibuya (1.3) (que não depende do tempo) pode ser escrita como

$$
\Lambda_{0}\left(F_{1}(x), F_{2}(y)\right)=\frac{F(x, y)}{F_{1}(x) F_{2}(y)}, \forall(x, y) \in \mathfrak{R}^{2}, \forall t \in Z, \operatorname{com} F_{1}(x)>0 \text { e } F_{2}(y)>0,
$$

ou então

$$
\Lambda_{0}(u, v)=\frac{C(u, v)}{u v}, \forall(u, v) \in(0,1]^{2}, \forall t \in Z,
$$

em que

$$
\begin{aligned}
& C(u, v)=F\left(F_{1}^{-1}(u), F_{2}^{-1}(v)\right), \\
& F_{1}^{-1}(u)=\inf \left\{x \in \mathfrak{R}: F_{1}(x) \geq u\right\}, \\
& F_{2}^{-1}(v)=\inf \left\{y \in \mathfrak{R}: F_{2}(y)=v\right\} .
\end{aligned}
$$

Denominamos (4.4) e (4.5) de função de dependência cruzada contemporânea local, sob o enfoque de funções de distribuição ou de cópula, respectivamente.

Para esse processo, as propriedades (i) a (v) da seção 1.2.3 são satisfeitas por (4.4), e também por (4.5) com as devidas adaptações.

\subsubsection{Estimador}

Aqui, propomos os mesmos tipos de estimadores suavizados que sugerimos para o caso de variáveis aleatórias. Observados $\left(\left(X_{1}, Y_{1}\right), \ldots,\left(X_{T}, Y_{T}\right)\right)$ do processo sob estudo, temos que um estimador para (4.4) é

$$
\hat{\Lambda}_{0}\left(\hat{F}_{1}(x), \hat{F}_{2}(y)\right)=\frac{\hat{F}(x, y)}{\hat{F}_{1}(x) \hat{F}_{2}(y)},
$$

em que

$$
\begin{aligned}
& \hat{F}(x, y)=\frac{1}{T} \sum_{t=1}^{T} K\left(\frac{x-X_{t}}{h_{1}}, \frac{y-Y_{t}}{h_{2}}\right), \\
& \hat{F}_{1}(x)=\frac{1}{T} \sum_{t=1}^{T} K_{1}\left(\frac{x-X_{t}}{h_{1}}\right), \\
& \hat{F}_{2}(y)=\frac{1}{T} \sum_{t=1}^{T} K_{2}\left(\frac{y-Y_{t}}{h_{2}}\right),
\end{aligned}
$$

sendo $h_{1}, h_{2}, K, K_{1}$ e $K_{2}$ como antes. Já o estimador de (4.5) é representado por

$$
\hat{\Lambda}_{0}(u, v)=\frac{\hat{C}(u, v)}{u v}, \forall(u, v) \in(0,1]^{2},
$$

em que 


$$
\begin{aligned}
& \hat{C}(u, v)=\hat{F}\left(\hat{F}_{1}^{-1}(u), \hat{F}_{2}^{-1}(v)\right), \\
& \hat{F}_{1}^{-1}(u)=\inf \left\{x \in \mathfrak{R}: \hat{F}_{1}(x) \geq u\right\}, \\
& \hat{F}_{2}^{-1}(v)=\inf \left\{y \in \mathfrak{R}: \hat{F}_{2}(y) \geq v\right\} .
\end{aligned}
$$

\subsubsection{Consistência dos estimadores}

Sejam as seguintes condições de regularidade, em que $h_{*}=\operatorname{máx}\left(h_{1}, h_{2}\right)$ com $h_{i} \rightarrow 0, i=1,2$, conforme $T \rightarrow \infty$.

(C1) $T h_{*}^{2} \rightarrow 0$ quando $T \rightarrow \infty$;

(C1') $T h_{*}^{4} \rightarrow 0$ quando $T \rightarrow \infty$, e o kernel bivariado $k$ é par;

(C2) o kernel $k$ tem suporte compacto;

(C3) o processo $\left(X_{t}, Y_{t}\right)$ é $\alpha$-mixing com coeficientes $\alpha_{T}=o\left(T^{-a}\right)$ para algum $a>1$, conforme $T \rightarrow \infty$;

(C4) as distribuições marginais $F_{i}, i=1,2$, são continuamente deriváveis nos respectivos intervalos $\left[F_{i}^{-1}(a)-\varepsilon ; F_{i}^{-1}(b)+\varepsilon\right]$, para todo $0<a<b<1$ e algum $\varepsilon>0$, com derivadas positivas $f_{i}$. Mais ainda, as derivadas parciais de ordem um de $F$ existem e são contínuas Lipschitzianas no produto desses intervalos.

Teorema 4.4. Seja $\left\{\left(X_{t}, Y_{t}\right), t \in Z\right\}$ um processo estacionário estrito com as condições $(\mathrm{C} 1)\left(\mathrm{ou}\left(\mathrm{C} 1^{\prime}\right)\right)$ a $(\mathrm{C} 4)$ satisfeitas. Então,

$$
\hat{\Lambda}_{0}\left(\hat{F}_{1}(x), \hat{F}_{2}(y)\right) \underset{T \rightarrow \infty}{\stackrel{P}{\longrightarrow}} \Lambda_{0}\left(F_{1}(x), F_{2}(y)\right) \text {, para cada }(x, y) \in \mathfrak{R}^{2} \text { fixado, }
$$

$\operatorname{com} \hat{F}_{1}(x), \hat{F}_{2}(y)>0$ e $F_{1}(x), F_{2}(y)>0$.

prova:

Satisfeitas as condições (C1) (ou (C1')) a (C4), então o Teorema 2 de Fermanian e Scaillet (2003) é válido, ou seja, $T^{1 / 2} \sup _{(x, y)}|\hat{F}(x, y)-F(x, y)|=o_{P}(1)$. Então, $\hat{F}(x, y) \stackrel{P}{\longrightarrow} F(x, y)$ quando $T \rightarrow \infty$. E como as distribuições marginais são funções da distribuição conjunta, então pelo Teorema 5.1.4. de Fuller, temos que $\hat{F}_{i}(x) \stackrel{P}{\longrightarrow} F_{i}(x), i=1,2$, conforme $T \rightarrow \infty$.

Sabemos pelo Lema 5.1.3 e Teorema 5.1.4 de Fuller (1976), que uma função contínua (com denominador não nulo) de estimadores que convergem em probabilidade para o verdadeiro parâmetro, também converge em probabilidade para a função contínua (com denominador não nulo) dos parâmetros. Então $\hat{\Lambda}_{0}\left(\hat{F}_{1}(x), \hat{F}_{2}(y)\right) \stackrel{P}{\longrightarrow} \Lambda_{0}\left(F_{1}(x), F_{2}(y)\right)$, conforme $T \rightarrow \infty$, para cada $(x, y) \in \mathfrak{R}^{2}$ fixado.

Teorema 4.5. Satisfeitas as condições $(\mathrm{C} 1)\left(\mathrm{ou}\left(\mathrm{C} 1^{\prime}\right)\right)$ a (C4) anteriores, então

$$
\hat{\Lambda}_{0}(u, v) \underset{T \rightarrow \infty}{\stackrel{P}{\longrightarrow}} \Lambda_{0}(u, v) \text {, para cada }(u, v) \in(0,1]^{2} \text { fixado. }
$$


prova:

Satisfeitas as condições mencionadas, então o Teorema 3 de Fermanian e Scaillet (2003) é válido, ou seja, para $T \rightarrow \infty$, o processo suavizado $\sqrt{T}(\hat{C}(u, v)-C(u, v))$ tende fracamente para um processo Gaussiano em $l^{\infty}\left([0,1]^{2}\right)$ munido com norma do supremo. Portanto, $\hat{C}(u, v) \stackrel{P}{\longrightarrow} C(u, v)$, e então obtemos o resultado.

Observamos que a condição (C2) pode ser relaxada, controlando-se as caudas de $k$, por exemplo, supondo que $\sup _{j}\left|k_{j}(x)\right| \leq(1+|x|)^{-\alpha}$ para todo $x$ e algum $\alpha>0$. Esta suposição é satisfeita por vários kernels, inclusive o Gaussiano (veja Robinson, 1983).

\subsubsection{Simulações}

A função de dependência cruzada contemporânea local $\Lambda_{0}$ foi avaliada através de simulações da função teórica e estimada (via (4.4) e (4.6) inicialmente, e depois com (4.5) e (4.7)) do modelo bivariado VAR(1) estacionário, calculando-se as estatísticas usuais do estimador. Para isto, utilizamos 200 réplicas de Monte Carlo com correlação contemporânea 0,70 e nula, para séries com diferentes tamanhos. Novamente, utilizamos grade com $31 \times 31$ pontos, estimador $\hat{\Lambda}_{0}$ com kernel produto, $90 \%$ dos dados centrais, kernel Gaussiano e largura de faixa ótima de Hansen (2004).

Inicialmente, consideramos o modelo autoregressivo vetorial estacionário de ordem um dado por

$$
\mathbf{Z}_{t}=\boldsymbol{\Phi}_{0}+\boldsymbol{\Phi}_{1} \mathbf{Z}_{t-1}+\boldsymbol{\varepsilon}_{t}
$$

em que $\mathbf{Z}_{t}=\left(X_{t}, Y_{t}\right), \boldsymbol{\Phi}_{0}=(1,1)^{\prime}, \operatorname{vec}\left(\boldsymbol{\Phi}_{1}\right)=(0,25 ; 0,2 ; 0,2 ; 0,75)^{\prime}$ e $\boldsymbol{\varepsilon}_{t} \sim N(\mathbf{0}, \boldsymbol{\Sigma})$ com $\operatorname{vec}(\boldsymbol{\Sigma})=(0,75 ; 0,5 ; 0,5 ; 1,25)^{\prime}$, ou seja, $\mathbf{Z}_{t}$ tem componentes dependentes. Portanto, os parâmetros da distribuição Gaussiana estacionária são $\boldsymbol{\mu}=(3,05 ; 6,44)^{\prime}$ e $\operatorname{vec}(\boldsymbol{\Gamma}(0))=(1,13 ; 1,49 ; 1,49 ; 3,99)^{\prime}$ (correlação 0,70). Então, a função teórica $\Lambda_{0}$ dada por (4.4) foi construída com base nesta distribuição, e a função estimada $\hat{\Lambda}_{0}$ dada por (4.6) foi elaborada através de 200 réplicas de Monte Carlo com tamanho 1.000, observadas desse modelo VAR(1). Na Figura 4.13, encontram-se os gráficos da (a) função de dependência cruzada contemporânea local $\Lambda_{0}$ e de suas respectivas (b) curvas de nível, além da (c) função $\hat{\Lambda}_{0}$ e suas correspondentes (d) curvas de nível. Comparando os dois primeiros gráficos com os dois últimos, verificamos a ocorrência de uma pequena subestimação da medida para os menores valores da grade. Observando a Tabela 4.5, verificamos o viés e o erro quadrático médio, além do $p$-valor do teste de normalidade de Jarque Bera que rejeitou a normalidade nos quatro primeiros pontos de grade avaliados. Na Figura 4.14, apresentam-se os histogramas das funções estimadas, os quais indicam distribuição aproximadamente normal.

Repetimos estas simulações para séries de tamanho 50, 100, 200 e 500, cujos resultados principais apresentam-se na Figura D.5 do Apêndice D. Comparando-se as cinco simulações com diferentes tamanhos de séries, verificamos que com o aumento do tamanho da série, o viés geralmente diminui, diminuindo também o erro quadrático médio e a rejeição da normalidade, mas esta ocorre de forma muito lenta. Somente na simulação com $T=1.000$ é que observamos a não rejeição da normalidade $(\alpha=5 \%)$ nos três últimos pontos de grade. 
Agora, vamos avaliar o modelo VAR(1) com as componentes de $\mathbf{Z}_{t}$ independentes, no caso, com $\boldsymbol{\Phi}_{0}=(1,1)^{\prime}, \quad \operatorname{vec}\left(\boldsymbol{\Phi}_{1}\right)=(0,25 ; 0 ; 0 ; 0,75)^{\prime} \quad$ e $\operatorname{vec}(\boldsymbol{\Sigma})=(0,75 ; 0 ; 0 ; 1,25)^{\prime}$. Então, os parâmetros da distribuição Gaussiana estacionária são $\boldsymbol{\mu}=(1,33 ; 4)^{\prime}$ e $\operatorname{vec}(\boldsymbol{\Gamma}(0))=(0,8 ; 0 ; 0 ; 2,86)^{\prime}$ (correlação nula). Novamente, para avaliarmos o estimador (4.6), construímos $\Lambda_{0}$ utilizando esta distribuição e geramos 200 séries de tamanho 1.000 a partir deste modelo. Na Figura 4.15 temos os gráficos (a) e (c), respectivamente da função $\Lambda_{0}$ teórica e estimada (além de seus respectivos gráficos de curvas de nível), os quais indicam comportamento muito similar. Na Tabela 4.6, apresentam-se as estatísticas básicas do estimador. Notamos que a normalidade não foi rejeitada. Na Figura 4.16 temos os respectivos histogramas.

Avaliando simulações similares, mas agora com séries de tamanho 50, 100, 200 e 500 (veja Figura D.6 do Apêndice D), verificamos que com o aumento do tamanho da série, o viés (em módulo) e o erro quadrático médio geralmente diminuem, e a rejeição de normalidade não aumenta.

Comparando as simulações de correlação contemporânea 0,70 com as de correlação nula (para os cinco tamanhos de série avaliados), verificamos que o viés, o erro quadrático médio e a rejeição da normalidade diminuem com a redução da correlação.

As simulações aqui realizadas para processos estocásticos são simulações correspondentes àquelas elaboradas para variáveis aleatórias (seção 4.1), no sentido da distribuição dos dados ser Gaussiana com $\boldsymbol{\mu}=(3,05 ; 6,44)^{\prime}$ e $\operatorname{vec}(\boldsymbol{\Gamma}(0))=(1,13 ; 1,49 ; 1,49 ; 3,99)^{\prime}$ (correlação 0,70$)$, e $\boldsymbol{\mu}=(1,33 ; 4)^{\prime}$ e $\operatorname{vec}(\boldsymbol{\Gamma}(0))=(0,80 ; 0 ; 0 ; 2,86)^{\prime}$ (correlação 0,0). Além disso, as amostras observadas e a quantidade de repetições são as mesmas, assim como os demais parâmetros de simulação. Então, comparando as simulações para variáveis aleatórias com as realizadas para processos estocásticos, verificamos:

(i) simulação com correlação 0,70 : no caso de variáveis aleatórias, o estimador apresenta viés (em módulo) geralmente maior e não rejeição de normalidade maior. Já o erro quadrático médio geralmente é maior para séries temporais. Porém, com o aumento do tamanho da amostra, essas quantidades passam a ser menos diferentes;

(ii) simulação com correlação nula: de modo geral, o viés (em módulo), o erro quadrático médio e a rejeição da normalidade do estimador é maior para processos estocásticos do que para variáveis aleatórias, porém, para tamanhos de amostra já a partir de 200, estas diferenças são muito pequenas.

Estas conclusões são similares às obtidas por Morettin et al. (2006), em que a estrutura de dependência (cópula) estimada através de ondaletas para a série simulada como um todo, é similar à estrutura de dependência estimada considerando diversas subséries amostradas daquela, de modo a reduzir a estrutura de dependência temporal. Observamos que estes autores utilizaram o modelo VAR(1) com correlação contemporânea 0,70 , mencionado anteriormente.

A seguir, avaliamos a função de dependência de Sibuya dada por (4.5) utilizando o estimador (4.7).

Considerando o modelo VAR(1) anterior com correlação 0,70, vemos através da Figura 4.17 que há leve subestimação da função. Na Tabela 4.7, vemos que o viés e o erro quadrático médio apresentam valores consideravelmente maiores no primeiro ponto de grade. Também, por esta tabela, vemos que a rejeição de normalidade ocorre em dois pontos de grade. Na Figura 4.18, temos os histogramas correspondentes. 
Comparamos esta simulação com outras de tamanho 50,100 e 200, cujos resultados apresentam-se na Figura D.7 do Apêndice D. Vemos que com o aumento de $T$, o viés e o erro quadrático médio diminuem, e a rejeição de normalidade parece oscilar menos.

Este estimador quando comparado com o estimador anterior, geralmente apresenta viés um pouco maior, e erro quadrático médio e rejeição de normalidade menores.

Para o modelo VAR(1) com correlação nula avaliado anteriormente, vemos o comportamento da função nos gráficos da Figura 4.19 e também na Tabela 4.8, as quais indicam leve subestimação, além de destacado erro quadrático médio no primeiro ponto de grade. Além disso, a normalidade foi rejeitada nos dois pontos extremos da grade. Os histogramas do estimador apresentam-se na Figura 4.20.

Simulações com tamanhos de série 50, 100 e 200 foram elaboradas e comparadas com esta (Figura D.8 do Apêndice D). Observamos que com o aumento de $T$, o viés e o erro quadrático médio geralmente diminuem, e a rejeição de normalidade parece diminuir para $T=500$.

Comparando os dois estimadores, vemos que este apresenta viés e erro quadrático médio geralmente menores, e a rejeição de normalidade é menor exceto para $T=500$.

Agora, comparando as funções de dependência com correlação 0,70 e nula estimadas através de (4.7), vemos que na função estimada com correlação nula, o viés e o erro quadrático médio geralmente são menores, e a rejeição da normalidade é maior.

Avaliando o comportamento das simulações para variáveis aleatórias e para séries temporais considerando $\Lambda_{0}$ estimada através de (4.7), vemos que no caso de séries temporais, tanto para correlação 0,70 quanto para correlação nula, o viés e o erro quadrático médio são geralmente um pouco maiores (principalmente para amostras pequenas e com correlação nula), e a rejeição de normalidade não difere substancialmente. 


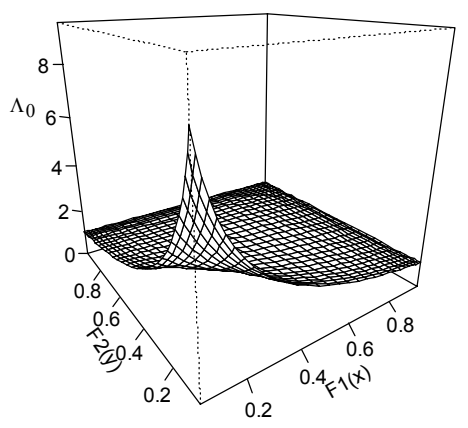

(a)

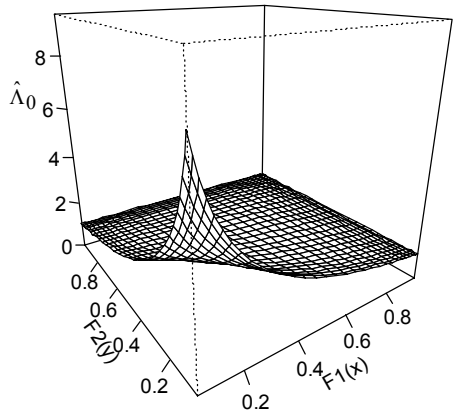

(c)

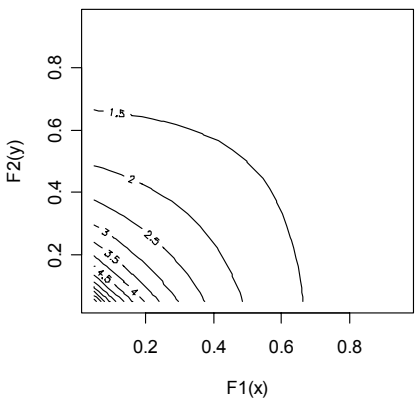

(b)

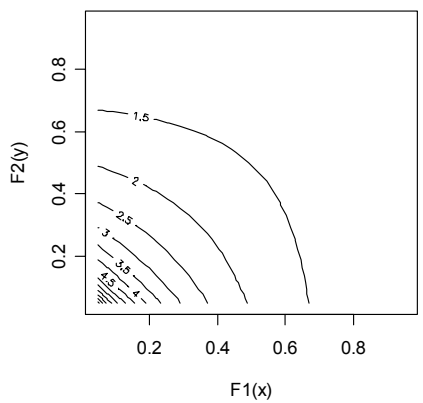

(d)

Figura 4.13 - Para o modelo VAR(1) com média $(3,05 ; 6,44)^{\prime}, \operatorname{vec}(\Gamma(0))=(1,13 ; 1,49 ; 1,49 ; 3,99)^{\prime}$ (correlação 0,70$)$ e inovações Gaussianas, temos o (a) gráfico de $\Lambda_{0}$ via funções de distribuição e respectivo (b) gráfico de curvas de nível, além do (c) gráfico de $\hat{\Lambda}_{0}$ e correspondente (d) gráfico de curvas de nível , para 200 séries com $T=1.000$.

Tabela 4.5 - Viés, erro quadrático médio e $p$-valor do teste de normalidade de Jarque Bera, para sete pontos de grade da diagonal principal de $\hat{\Lambda}_{0}$, considerando 200 séries de tamanho 1.000 , observadas do modelo VAR(1) com média $(3,05 ; 6,44)^{\prime}, \operatorname{vec}(\Gamma(0))=(1,13 ; 1,49 ; 1,49 ; 3,99)^{\prime}$ (correlação 0,70$)$ e inovações Gaussianas.

\begin{tabular}{|l|c|c|c|c|c|c|c|}
\hline \multicolumn{1}{|c|}{ Grade } & $\mathbf{0 , 0 5}$ & $\mathbf{0 , 1 4}$ & $\mathbf{0 , 2 9}$ & $\mathbf{0 , 5 0}$ & $\mathbf{0 , 7 1}$ & $\mathbf{0 , 8 6}$ & $\mathbf{0 , 9 5}$ \\
\hline Viés & $-0,299$ & $-0,088$ & $-0,018$ & $-0,004$ & 0,000 & 0,002 & 0,002 \\
\hline EQM & 2,079 & 0,168 & 0,027 & 0,005 & 0,001 & 0,000 & 0,000 \\
\hline $\boldsymbol{P}$-valor & 0,003 & 0,002 & 0,000 & 0,019 & 0,112 & 0,206 & 0,247 \\
\hline
\end{tabular}


0.05

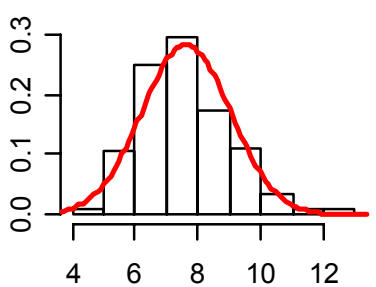

0.71

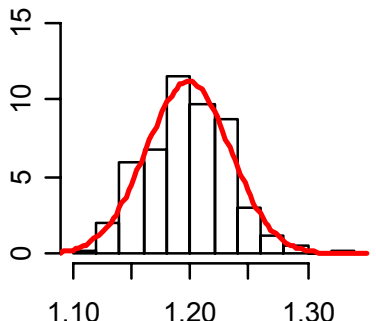

0.14

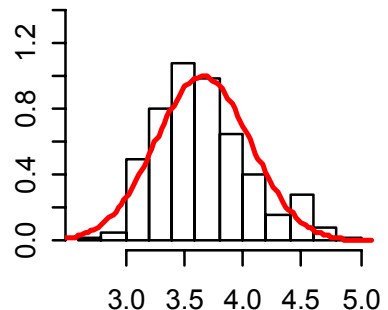

0.86

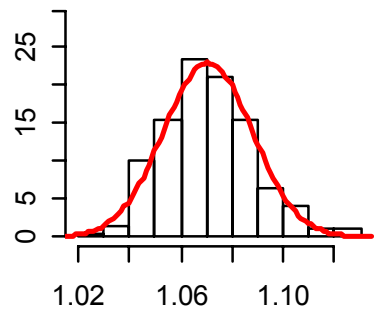

0.29

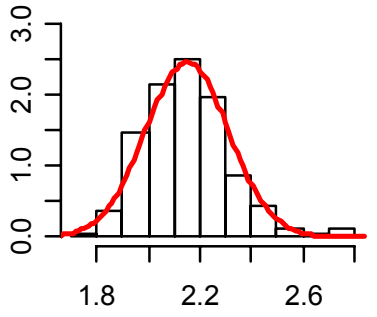

0.95

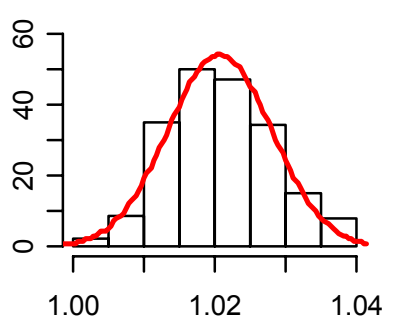

Figura 4.14 - Histogramas em pontos de grade da diagonal principal de $\hat{\Lambda}_{0}$, considerando 200 séries de tamanho 1.000, observadas do modelo VAR(1) com média $(3,05 ; 6,44)^{\prime}$, vec $(\Gamma(0))=(1,13 ; 1,49 ; 1,49 ; 3,99)^{\prime}$ (correlação 0,70$)$ e inovações Gaussianas. 


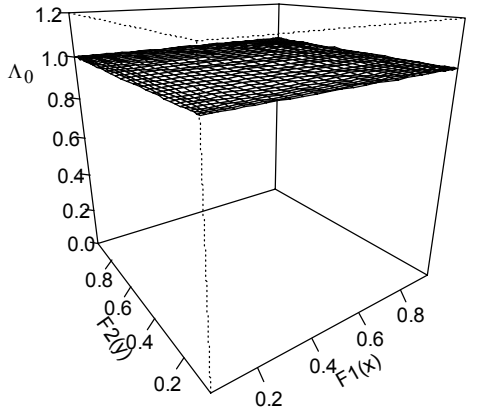

(a)

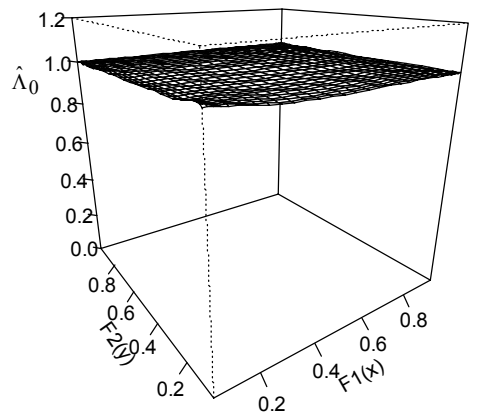

(c)

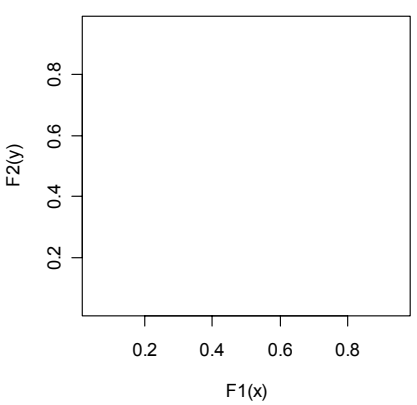

(b)

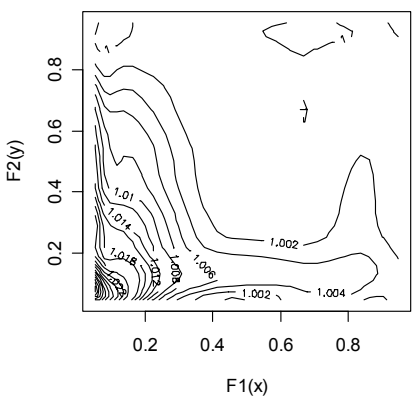

(d)

Figura 4.15 - Para o modelo VAR(1) com média $(1,33 ; 4)^{\prime}, \quad \operatorname{vec}(\Gamma(0))=(0,80 ; 0 ; 0 ; 2,86)^{\prime} \quad($ correlação 0,0$)$ e inovações Gaussianas, temos o (a) gráfico de $\Lambda_{0}$ via funções de distribuição e respectivo (b) gráfico de curvas de nível, além do (c) gráfico de $\hat{\Lambda}_{0}$ e correspondente (d) gráfico de curvas de nível, para 200 séries com $T=1.000$.

Tabela 4.6 - Viés, erro quadrático médio e $p$-valor do teste de normalidade de Jarque Bera, para sete pontos de grade da diagonal principal de $\hat{\Lambda}_{0}$, considerando 200 séries de tamanho 1.000 , observadas do modelo VAR(1) com média $(1,33 ; 4)^{\prime}, \operatorname{vec}(\Gamma(0))=(0,80 ; 0 ; 0 ; 2,86)^{\prime}$ (correlação 0,0$)$ e inovações Gaussianas.

\begin{tabular}{|l|c|c|c|c|c|c|c|}
\hline \multicolumn{1}{|c|}{ Grade } & $\mathbf{0 , 0 5}$ & $\mathbf{0 , 1 4}$ & $\mathbf{0 , 2 9}$ & $\mathbf{0 , 5 0}$ & $\mathbf{0 , 7 1}$ & $\mathbf{0 , 8 6}$ & $\mathbf{0 , 9 5}$ \\
\hline Viés & 0,036 & 0,023 & 0,006 & 0,001 & 0,000 & 0,000 & 0,000 \\
\hline EQM & 0,237 & 0,035 & 0,006 & 0,001 & 0,000 & 0,000 & 0,000 \\
\hline $\boldsymbol{P}$-valor & 0,363 & 0,364 & 0,270 & 0,701 & 0,705 & 0,052 & 0,085 \\
\hline
\end{tabular}


0.05

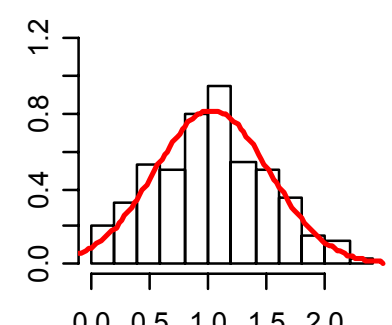

$\begin{array}{lllll}0.0 & 0.5 & 1.0 & 1.5 & 2.0\end{array}$

0.71

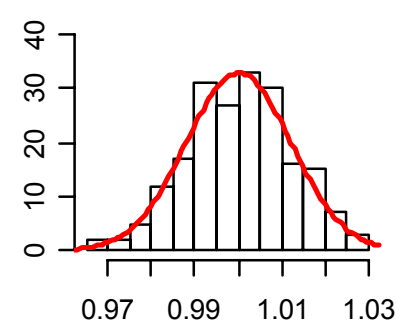

0.14

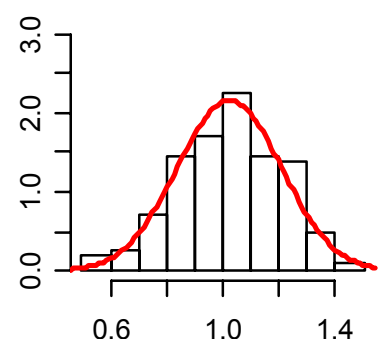

0.86

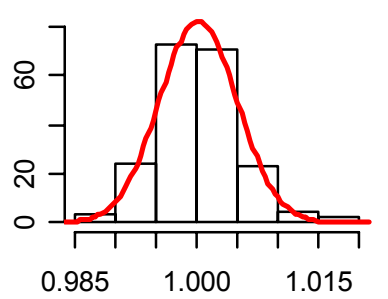

0.29

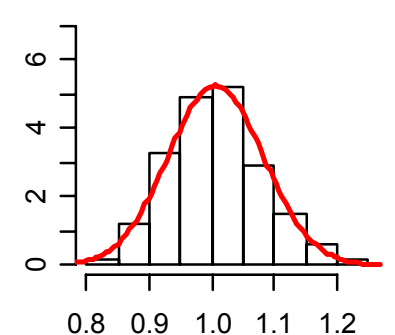

0.95

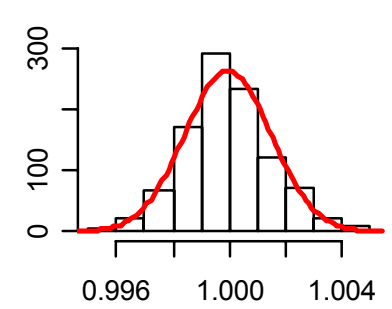

Figura 4.16 - Histogramas em pontos de grade da diagonal principal de $\hat{\Lambda}_{0}$, considerando 200 séries de tamanho 1.000, observadas do modelo $\operatorname{VAR}(1)$ com média $(1,33 ; 4)^{\prime}, \operatorname{vec}(\Gamma(0))=(0,80 ; 0 ; 0 ; 2,86)^{\prime}$ (correlação 0,0$)$ e inovações Gaussianas.. 


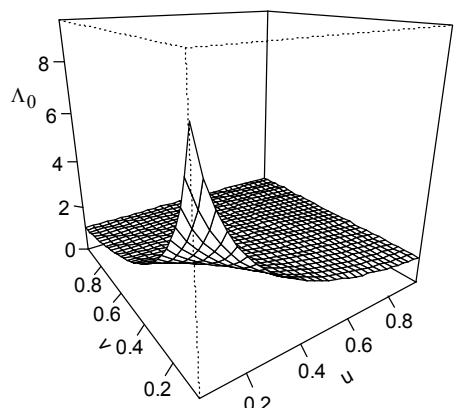

(a)

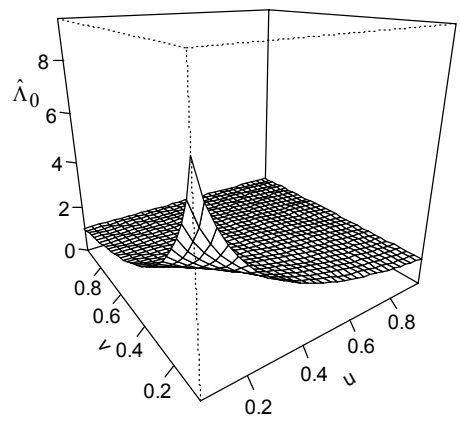

(c)

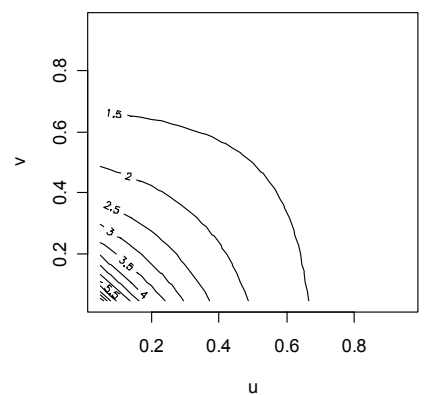

(b)

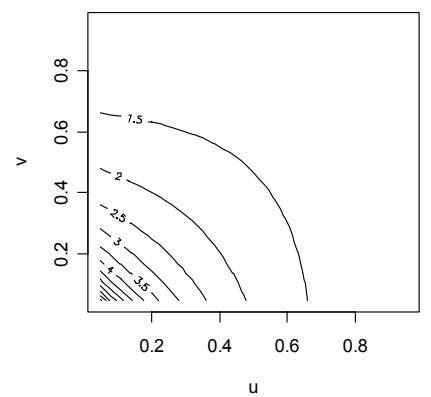

(d)

Figura 4.17 - Para o modelo VAR(1) com média $(3,05 ; 6,44)^{\prime}$, $\operatorname{vec}(\Gamma(0))=(1,13 ; 1,49 ; 1,49 ; 3,99)^{\prime}$ (correlação 0,70$)$ e inovações Gaussianas, temos (a) gráfico de $\Lambda_{0}$ via cópula e respectivo (b) gráfico de curvas de nível, além do (c) gráfico de $\hat{\Lambda}_{0}$ e correspondente (d) gráfico de curvas de nível, para 200 séries com $T=500$.

Tabela 4.7 - Viés, erro quadrático médio e $p$-valor do teste de normalidade de Jarque Bera, para sete pontos de grade da diagonal principal de $\hat{\Lambda}_{0}$, considerando 200 séries de tamanho 500, observadas do modelo VAR(1) com média $(3,05 ; 6,44)^{\prime}, \operatorname{vec}(\Gamma(0))=(1,13 ; 1,49 ; 1,49 ; 3,99)^{\prime}$ (correlação 0,70$)$ e inovações Gaussianas.

\begin{tabular}{|l|c|c|c|c|c|c|r|}
\hline Grade & $\mathbf{0 , 0 5}$ & $\mathbf{0 , 2 0}$ & $\mathbf{0 , 3 5}$ & $\mathbf{0 , 5 0}$ & $\mathbf{0 , 6 5}$ & \multicolumn{1}{c|}{$\mathbf{0 , 8 0}$} & \multicolumn{1}{c|}{$\mathbf{0 , 9 5}$} \\
\hline Viés & $-0,780$ & $-0,117$ & $-0,050$ & $-0,028$ & $-0,016$ & $-0,008$ & $-0,002$ \\
\hline EQM & 2,839 & 0,051 & 0,008 & 0,002 & 0,001 & 0,000 & 0,000 \\
\hline $\boldsymbol{P}$-valor & 0,891 & 0,176 & 0,109 & 0,044 & 0,001 & 0,813 & 0,607 \\
\hline
\end{tabular}



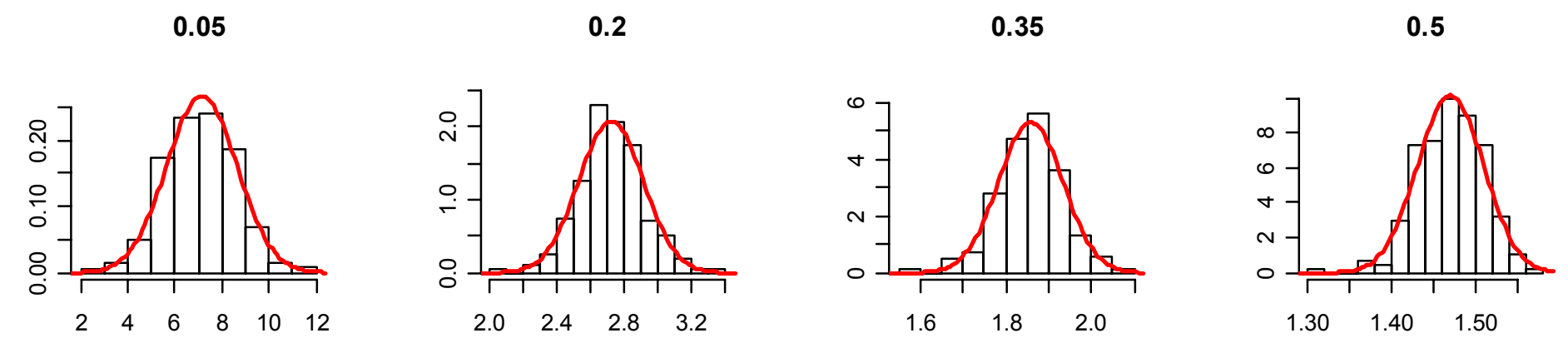

0.65

0.8

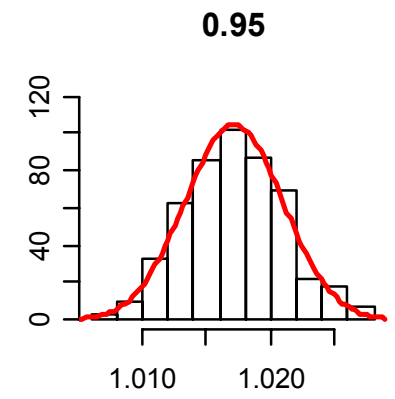

Figura 4.18 - Histogramas em pontos de grade da diagonal principal de $\hat{\Lambda}_{0}$, considerando 200 séries de tamanho 500, observadas do modelo $\operatorname{VAR}(1)$ com média $(3,05 ; 6,44)^{\prime}, \operatorname{vec}(\Gamma(0))=(1,13 ; 1,49 ; 1,49 ; 3,99)^{\prime}$ (correlação 0,70$)$ e inovações Gaussianas. 


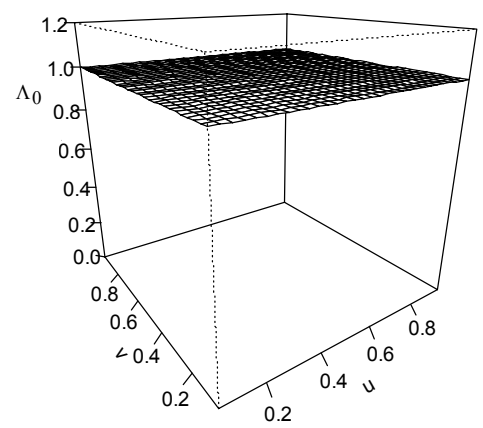

(a)

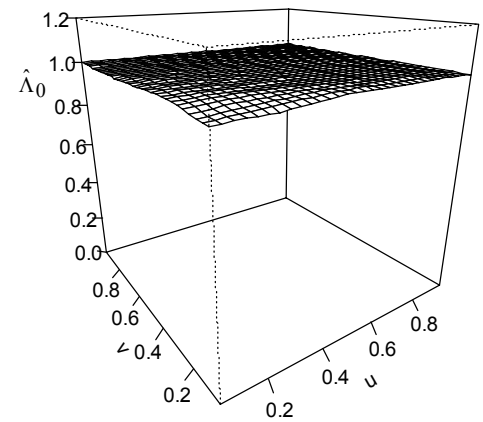

(c)

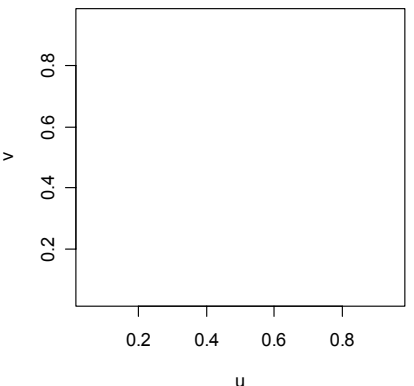

(b)

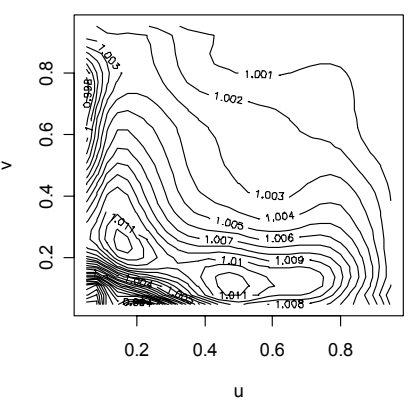

(d)

Figura 4.19 - Para o modelo VAR(1) com média $(1,33 ; 4)^{\prime}, \operatorname{vec}(\Gamma(0))=(0,80 ; 0 ; 0 ; 2,86)^{\prime}$ (correlação 0,0$)$ e inovações Gaussianas, temos o (a) gráfico de $\Lambda_{0}$ via cópula e respectivo (b) gráfico de curvas de nível, além do (c) gráfico de $\hat{\Lambda}_{0}$ e correspondente (d) gráfico de curvas de nível, para 200 séries com $T=500$.

Tabela 4.8 - Viés, erro quadrático médio e $p$-valor do teste de normalidade de Jarque Bera, para sete pontos de grade da diagonal principal de $\hat{\Lambda}_{0}$, considerando 200 séries de tamanho 500, observadas do modelo VAR(1) com média $(1,33 ; 4)^{\prime}, \operatorname{vec}(\Gamma(0))=(0,80 ; 0 ; 0 ; 2,86)^{\prime}$ (correlação 0,0$)$ e inovações Gaussianas.

\begin{tabular}{|l|c|c|c|c|c|c|c|}
\hline Grade & $\mathbf{0 , 0 5}$ & $\mathbf{0 , 2 0}$ & $\mathbf{0 , 3 5}$ & $\mathbf{0 , 5 0}$ & $\mathbf{0 , 6 5}$ & $\mathbf{0 , 8 0}$ & $\mathbf{0 , 9 5}$ \\
\hline Viés & $-0,014$ & 0,012 & 0,005 & 0,003 & 0,002 & 0,001 & 0,000 \\
\hline EQM & 0,449 & 0,023 & 0,006 & 0,002 & 0,001 & 0,000 & 0,000 \\
\hline $\boldsymbol{P}$-valor & 0,000 & 0,199 & 0,657 & 0,637 & 0,537 & 0,550 & 0,000 \\
\hline
\end{tabular}


0.05

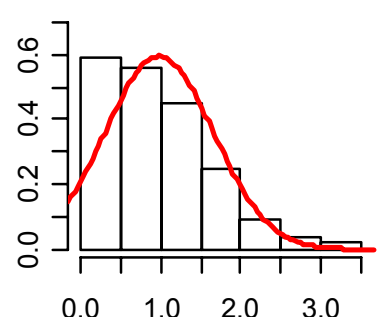

0.65

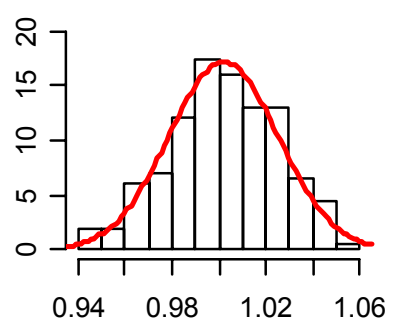

0.2

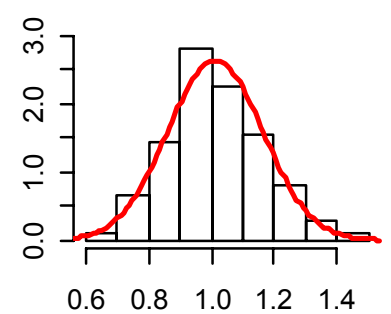

0.8

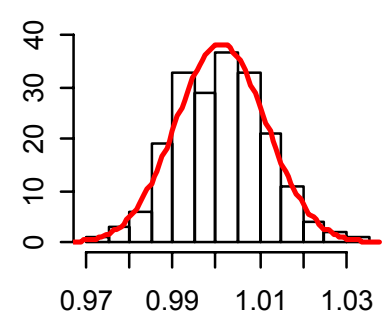

0.35

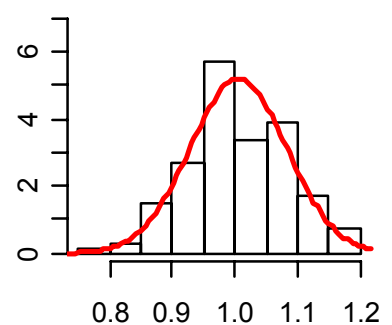

0.95

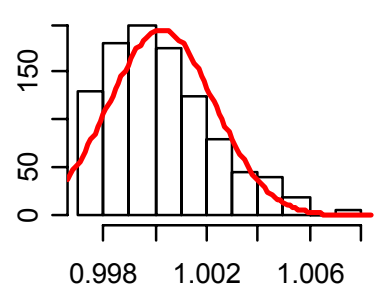

Figura 4.20 - Histogramas em pontos de grade da diagonal principal de $\hat{\Lambda}_{0}$, considerando 200 séries de tamanho 500, observadas do modelo VAR $(1)$ com média $(1,33 ; 4)^{\prime}, \operatorname{vec}(\Gamma(0))=(0,80 ; 0 ; 0 ; 2,86)^{\prime}$ (correlação 0,0$)$ e inovações Gaussianas. 


\subsubsection{Aplicações a séries reais}

A seguir, avaliamos a estrutura de dependência entre os retornos diários da Petrobrás e do Ibovespa, e também entre os retornos diários do Banespa e do Ibovespa, no período de 3/jan/95 a 27/dez/00 totalizando 1.498 dados. Os parâmetros da simulação são os mesmos utilizados na seção 4.1.4, mas agora consideramos $90 \%$ dos dados centrais. Além disso, inicialmente avaliamos esses dados utilizando o estimador (4.6) e depois o (4.7).

Vimos na seção 2.2.2, que os retornos da Petrobrás $\left(X_{t}\right)$ e do Ibovespa $\left(Y_{t}\right)$ apresentaram correlação linear de Pearson de 0,83 , além de dependência intra (f.a.c. dos retornos ao quadrado) e inter (f.a.c.c. dos retornos ao quadrado) séries. A Figura 4.21 a seguir considera estas séries, sendo que o gráfico (a) indica forte relação linear positiva, em (b) e (c) vemos a função de dependência cruzada contemporânea local estimada via (4.6), a qual indica que as séries são comonotônicas. O gráfico (d) indica a existência de forte dependência positiva entre valores baixos e entre valores altos dos postos normalizados. Já nos gráficos (e), da cópula estimada via kernel, e (f), das respectivas curvas de nível, podemos observar a existência de forte dependência positiva, sendo que a intensidade desta é mais facilmente observada através do último gráfico mencionado.

Os retornos do Banespa e do Ibovespa apresentaram correlação linear 0,54, além de dependência intra e inter séries (seja seção 2.2.2). No gráfico (a) da Figura 4.22, observamos relação linear positiva entre os retornos das duas séries, e nos gráficos (b) e (c) da função de dependência cruzada contemporânea local estimada através de (4.6), vemos que os retornos são comonotônicos, porém com menor intensidade que o caso acima. O gráfico (d) mostra dependência positiva entre os baixos valores e entre os altos valores dos postos normalizados. Também, os gráficos (e) e (f) mostram dependência positiva.

Utilizando o estimador da função de dependência cruzada contemporânea local dado por (4.7), vemos através da Figura 4.23 que os retornos diários da Petrobrás $\left(X_{t}\right)$ e do Ibovespa $\left(Y_{t}\right)$ são comonotônicos, assim como os retornos diários do Banespa e do Ibovespa (Figura 4.24), embora com menor intensidade.

Comparando os gráficos (b) e (c) da Figura 4.21 (4.22) com os da Figura 4.23 (4.24), vemos que o comportamento é muito similar (similar). 


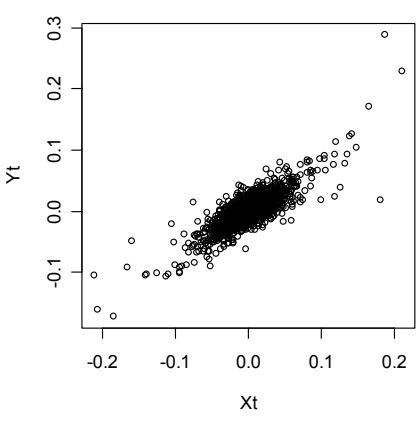

(a)

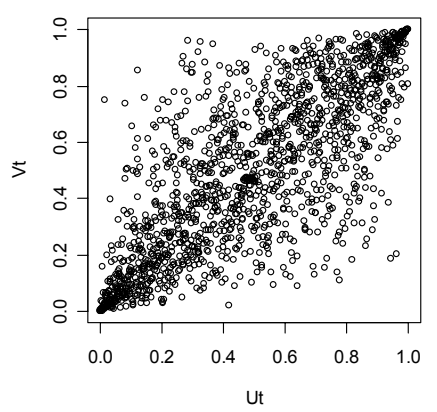

(d)

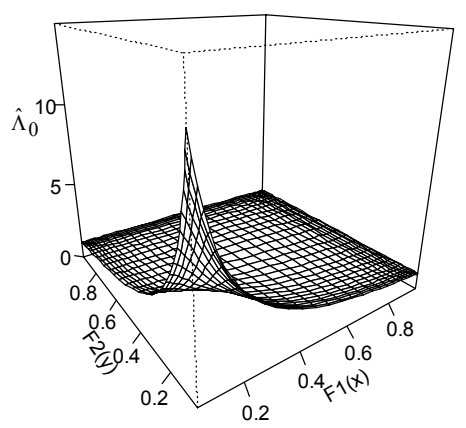

(b)

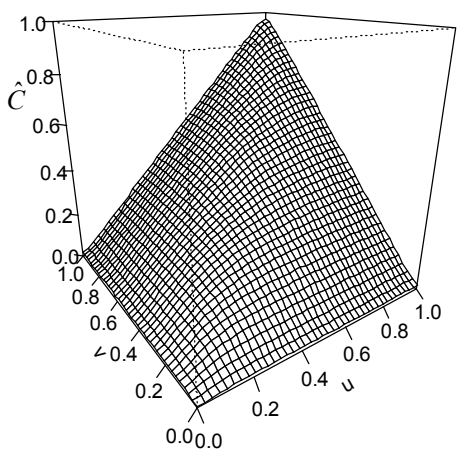

(e)

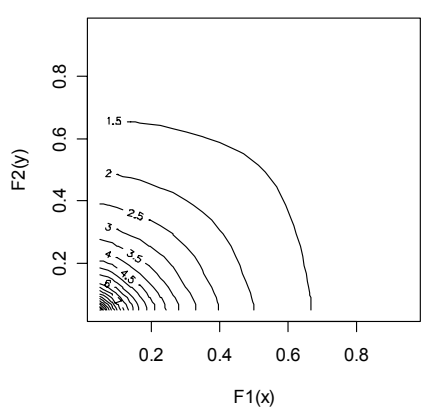

(c)

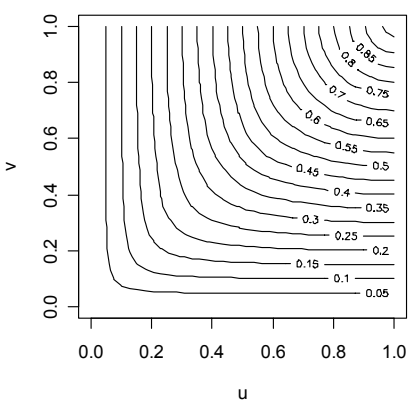

(f)

Figura 4.21 - Para os retornos diários da Petrobrás $\left(X_{t}\right)$ e do Ibovespa $\left(Y_{t}\right)$, de 3/jan/95 a 27/dez/00, temos: (a) gráfico de dispersão, (b) $\hat{\Lambda}_{0}$ via funções de distribuição e respectivo (c) gráfico de curvas de nível. Para os correspondentes postos normalizados, temos o (d) gráfico de dispersão, (e) cópula suavizada e respectivas (f) curvas de nível. 


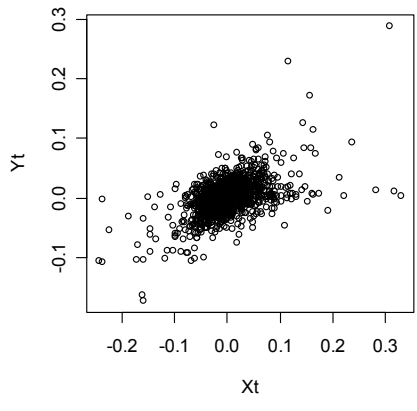

(a)

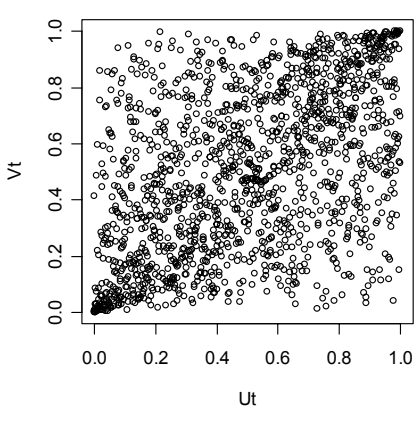

(d)

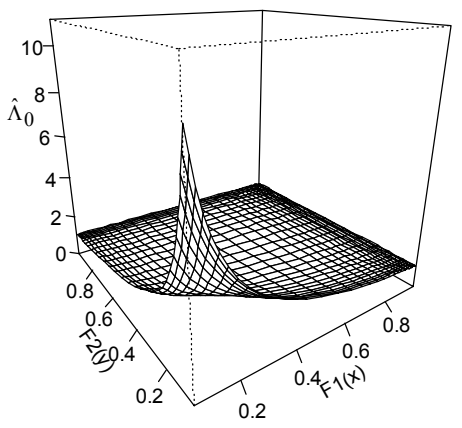

(b)

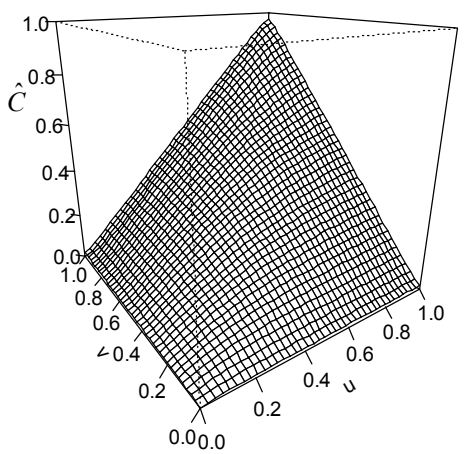

(e)

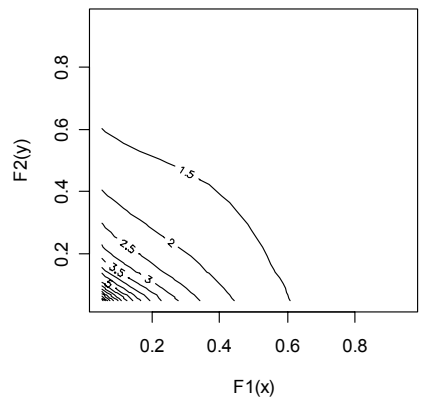

(c)

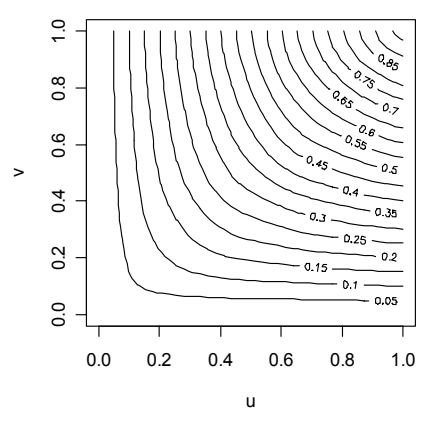

(f)

Figura 4.22 - Para os retornos diários da Banespa $\left(X_{t}\right)$ e do Ibovespa $\left(Y_{t}\right)$, de 3/jan/95 a 27/dez/00, temos: (a) gráfico de dispersão, (b) $\hat{\Lambda}_{0}$ via funções de distribuição e respectivo (c) gráfico de curvas de nível. Para os correspondentes postos normalizados, temos o (d) gráfico de dispersão, (e) cópula suavizada e respectivas (f) curvas de nível. 


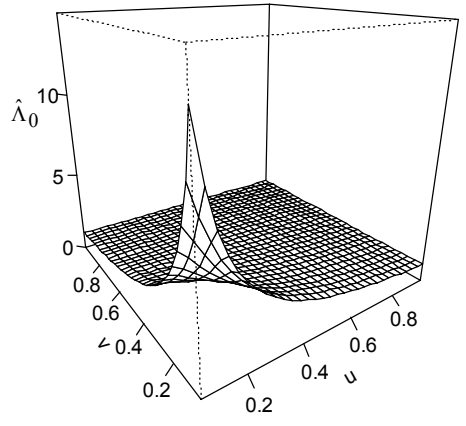

(a)

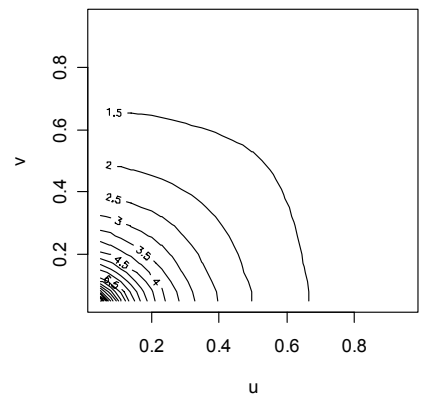

(b)

Figura 4.23 - Para os retornos diários da Petrobrás $\left(X_{t}\right)$ e do Ibovespa $\left(Y_{t}\right)$, de $3 /$ jan/95 a 27/dez/00, temos: (a) $\hat{\Lambda}_{0}$ via cópula e respectivo (b) gráfico de curvas de nível.

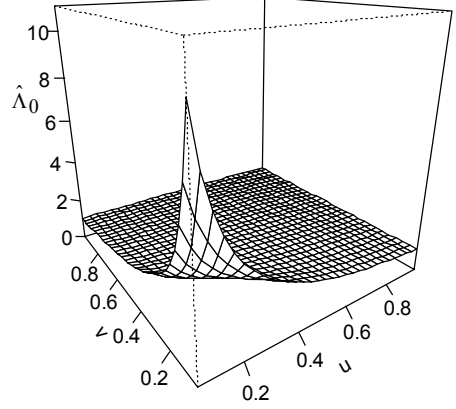

(a)

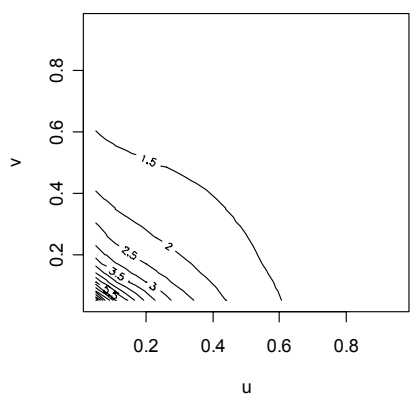

(b)

Figura 4.24 - Para os retornos diários da Banespa $\left(X_{t}\right)$ e do Ibovespa $\left(Y_{t}\right)$, de 3/jan/95 a 27/dez/00, temos: (a) $\hat{\Lambda}_{0}$ via cópula e respectivo (b) gráfico de curvas de nível. 


\subsection{Função de Autodependência Local}

Considere $\left\{X_{t}, t \in Z\right\}$ um processo estacionário estrito a valores contínuos. Conforme visto na seção 2.1, a distribuição univariada não depende do tempo e a distribuição de $\left(X_{t}, X_{t+\tau}\right), \forall t, \tau \in Z$, só depende da defasagem. Portanto, seja $F\left(x_{1}, x_{2} ; \tau\right)=P\left[X_{t} \leq x_{1}, X_{t+\tau} \leq x_{2}\right]$ a distribuição conjunta de $\left(X_{t}, X_{t+\tau}\right)$, com distribuição marginal $F\left(x_{i}\right), i=1,2$.

Então, considerando $Y_{t}=X_{t+\tau}$, temos que a função de dependência de Sibuya (4.4) pode ser reescrita como

$$
\Lambda_{\tau}\left(F\left(x_{1}\right), F\left(x_{2}\right)\right)=\frac{F\left(x_{1}, x_{2} ; \tau\right)}{F\left(x_{1}\right) F\left(x_{2}\right)}, \forall\left(x_{1}, x_{2}\right) \in \mathfrak{R}^{2}, \forall \tau \in Z, \operatorname{com} F\left(x_{i}\right)>0, i=1,2,
$$

e a função (4.5) como

$$
\Lambda_{\tau}\left(u_{1}, u_{2}\right)=\frac{C\left(u_{1}, u_{2}\right)}{u_{1} u_{2}}, \forall\left(u_{1}, u_{2}\right) \in(0,1]^{2}, \forall \tau \in Z,
$$

em que

$$
\begin{aligned}
& C\left(u_{1}, u_{2}\right)=F\left(F^{-1}\left(u_{1}\right), F^{-1}\left(u_{2}\right) ; \tau\right), \\
& F^{-1}\left(u_{i}\right)=\inf \left\{x_{i} \in \mathfrak{R}: F\left(x_{i}\right) \geq u_{i}\right\}, i=1,2 .
\end{aligned}
$$

Nomeamos de função de autodependência local as expressões (4.8) e (4.9) construídas com base em distribuições e cópula, respectivamente.

As propriedades (i), (iii), (iv)-a e (iv)-d (em que $\varphi()=.\psi($.$) ), além de (v) (neste caso, se \left\{X_{t}\right\}$ é processo Gaussiano) da seção 1.2.3 são satisfeitas por (4.8) e (4.9), com as devidas adaptações. Também, para estas funções são válidas as seguintes propriedades, as quais escrevemos em termos de (4.8) apenas por conveniência:

(a) $\Lambda_{\tau}\left(F\left(x_{1}\right), F\left(x_{2}\right)\right) \underset{\tau \rightarrow \infty}{\longrightarrow} 1, \forall\left(x_{1}, x_{2}\right) \in \mathfrak{R}^{2}, \tau \in Z$, se o processo é $\alpha$-mixing;

prova: Imediata.

(b) $\Lambda_{-\tau}\left(F\left(x_{1}\right), F\left(x_{2}\right)\right)=\Lambda_{\tau}\left(F\left(x_{1}\right), F\left(x_{2}\right)\right), \forall\left(x_{1}, x_{2}\right) \in \mathfrak{R}^{2}, \forall \tau \in Z$.

prova:

Como o processo é estacionário estrito, então $\left(X_{t}, X_{t+\tau}\right)$ e $\left(X_{t-\tau}, X_{t}\right)$ têm a mesma distribuição, ou seja, $F\left(x_{1}, x_{2} ; \tau\right)=F\left(x_{1}, x_{2} ;-\tau\right)$, e então

$$
\Lambda_{\tau}\left(F\left(x_{1}\right), F\left(x_{2}\right)\right)=\frac{F\left(x_{1}, x_{2} ; \tau\right)}{F\left(x_{1}\right) F\left(x_{2}\right)}=\frac{F\left(x_{1}, x_{2} ;-\tau\right)}{F\left(x_{1}\right) F\left(x_{2}\right)}=\Lambda_{-\tau}\left(F\left(x_{1}\right), F\left(x_{2}\right)\right) .
$$

\subsubsection{Estimador}

O estimador para (4.8) pode ser visto como sendo uma adaptação do estimador (4.6) para este processo, isto é 


$$
\hat{\Lambda}_{\tau}\left(\hat{F}\left(x_{1}\right), \hat{F}\left(x_{2}\right)\right)=\frac{\hat{F}\left(x_{1}, x_{2} ; \tau\right)}{\hat{F}\left(x_{1}\right) \hat{F}\left(x_{2}\right)}, \forall\left(x_{1}, x_{2}\right) \in \mathfrak{R}^{2}, \forall \tau \in \mathcal{Z}, \operatorname{com} \hat{F}\left(x_{i}\right)>0, i=1,2,
$$

em que

$$
\begin{aligned}
& \hat{F}\left(x_{1}, x_{2} ; \tau\right)=\frac{1}{T-\tau} \sum_{t=1}^{T-\tau} K\left(\frac{x_{1}-X_{t}}{h}, \frac{x_{2}-X_{t+\tau}}{h}\right), \\
& \hat{F}\left(x_{i}\right)=\frac{1}{T} \sum_{t=1}^{T} K_{1}\left(\frac{x_{i}-X_{t}}{h}\right), i=1,2 .
\end{aligned}
$$

Aqui, $h$ denota a largura de faixa $h_{T}$ tal que $h \rightarrow 0$ conforme $T \rightarrow \infty$, e $K$ e $K_{1}$ são como antes.

De modo similar, o estimador que sugerimos para (4.9) é similar ao estimador (4.7), mas com a devida modificação, ou seja

$$
\hat{\Lambda}_{\tau}\left(u_{1}, u_{2}\right)=\frac{\hat{C}\left(u_{1}, u_{2}\right)}{u_{1} u_{2}}, \forall\left(u_{1}, u_{2}\right) \in(0,1]^{2}, \forall \tau \in Z
$$

em que

$$
\begin{aligned}
& \hat{C}\left(u_{1}, u_{2}\right)=\hat{F}\left(\hat{F}^{-1}\left(u_{1}\right), \hat{F}^{-1}\left(u_{2}\right) ; \tau\right), \\
& \hat{F}^{-1}\left(u_{i}\right)=\inf \left\{x_{i} \in \mathfrak{R}: \hat{F}\left(x_{i}\right) \geq u_{i}\right\} .
\end{aligned}
$$

\subsubsection{Consistência dos estimadores}

Vamos verificar as propriedades do estimador $\hat{\Lambda}$.

Sejam as seguintes condições de regularidade.

(C1) $T h^{2} \rightarrow 0$ quando $T \rightarrow \infty$;

(C1') $T h^{4} \rightarrow 0$ quando $T \rightarrow \infty$, e o kernel bivariado $k$ é par;

(C2) o kernel $k$ tem suporte compacto;

(C3) o processo $\left(X_{t}\right)$ é $\alpha$-mixing com coeficientes $\alpha_{T}=o\left(T^{-a}\right)$ para algum $a>1$, conforme $T \rightarrow \infty$;

(C4) a distribuição marginal $F$ é continuamente derivável no intervalo $\left[F^{-1}(a)-\varepsilon ; F^{-1}(b)+\varepsilon\right]$, para todo $0<a<b<1$ e algum $\varepsilon>0$, com derivada positiva $f$. Mais ainda, as derivadas parciais de ordem um de $F_{\tau}$ existem e são contínuas Lipschitzianas no produto desse intervalo.

Teorema 4.6. Seja $\left\{X_{t}, t \in Z\right\}$ um processo estacionário estrito. Satisfeitas as as condições (C1) (ou (C1')) a (C4) anteriores, então

$$
\hat{\Lambda}_{\tau}\left(\hat{F}\left(x_{1}\right), \hat{F}\left(x_{2}\right)\right) \underset{T \rightarrow \infty}{\stackrel{P}{\longrightarrow}} \Lambda_{\tau}\left(F\left(x_{1}\right), F\left(x_{2}\right)\right) \text {, para cada }\left(x_{1}, x_{2}\right) \in \mathfrak{R}^{2} \text { fixado e } \tau \in Z,
$$

com $\hat{F}\left(x_{i}\right)>0$ e $F\left(x_{i}\right)>0, i=1,2$.

prova: 
Similar à prova do Teorema 4.4, mas agora considerando $F\left(x_{1}, x_{2} ; \tau\right), F\left(x_{1}\right)$ e $F\left(x_{2}\right)$ ao invés de $F(x, y)$, $F_{1}(x)$ e $F_{2}(y)$, o mesmo ocorrendo com os seus respectivos estimadores.

Observamos que a convergência quase certa da distribuição conjunta deste processo pode ser encontrada em Azevedo e Oliveira (2000).

Teorema 4.7. Satisfeitas as condições $(\mathrm{C} 1)\left(\mathrm{ou}\left(\mathrm{C} 1^{\prime}\right)\right)$ a $(\mathrm{C} 4)$ anteriores, então

$$
\hat{\Lambda}_{\tau}\left(u_{1}, u_{2}\right) \underset{T \rightarrow \infty}{\stackrel{P}{\longrightarrow}} \Lambda_{\tau}\left(u_{1}, u_{2}\right) \text {, para cada }\left(u_{1}, u_{2}\right) \in(0,1]^{2} \text { fixado e } \tau \in Z, .
$$

prova:

Similar ao Teorema 4.5.

\subsubsection{Simulações}

Os estimadores (4.10) e (4.11) da função de autodependência local, $\hat{\Lambda}_{\tau}, \tau=1,2,3,4$, foram avaliados através da simulação da função de autodependência local teórica dada por (4.8) e (4.9) e de seus respectivos estimadores dados por (4.10) e (4.11). Para tanto, utilizamos 200 réplicas de Monte Carlo de séries observadas de um processo Gaussiano estacionário com média nula, variância unitária e estrutura de autocorrelação autoregressiva com $\phi_{1}=0,70$, sendo que para o primeiro estimador utilizamos séries de tamanhos 50, 100, 200, 500 e 1.000, e para o segundo estimador séries de com $T$ igual a 50, 100, 200 e 500 . Então, calculamos as estatísticas básicas de cada estimador. Os parâmetros utilizados nas simulações de $\hat{\Lambda}_{\tau}$ foram os mesmos da seção 4.2.3.

Primeiramente avaliamos a função de autodependência local via funções de distribuição com séries de tamanho 1.000. Na Figura 4.25 podemos observar $\Lambda_{\tau}, \tau=1,2,3,4$, através de gráficos de perspectiva e de curvas de nível, os quais indicam um decaimento da dependência ao longo dos lags. Na Figura 4.26 observamos os mesmos tipos de gráficos mas agora para $\hat{\Lambda}_{\tau}, \tau=1,2,3,4$, os quais mostram um comportamento muito similar ao teórico. Nas tabelas 4.9 a 4.11, vemos o viés, o erro quadrático médio e o $p$ valor do teste de normalidade de Jarque Bera do estimador (nos quatro primeiros lags) calculados em alguns pontos bivariados de grade da diagonal principal. Podemos verificar que há pequena subestimação, a qual para cada lag, diminui ao longo dos pontos da diagonal da grade, e também diminui ao longo dos lags para cada ponto de grade avaliado.O erro quadrático médio também diminui como o aumento do ponto de grade ou com o aumento do lag. Quanto a normalidade do estimador, no lag 1 ocorre rejeição da normalidade apenas no primeiro ponto de grade. Nos demais lags, a incidência de rejeição de normalidade é maior e não muito diferente entre as defasagens 2 a 4 . Na Figura 4.27 vemos os histogramas do estimador em alguns pontos de grade, os quais indicam normalidade exceto no primeiro ponto de grade.

Repetimos estas simulações para diferentes tamanhos de séries, cujos resultados principais para os lags 1 a 4, apresentam-se nas figuras D.9 a D.12 do Apêndice D. Analisando os resultados das simulações, para cada lag, verificamos que o viés e o erro quadrático médio diminuem com o aumento do tamanho da série, e a não 
rejeição da normalidade passa a ocorrer a partir de séries de tamanho 500 sendo maior na série de tamanho 1.000. Além disso, considerando cada tamanho de série, vemos que o viés geralmente diminui ao longo dos lags, o erro quadrático médio diminui no lag 1 e geralmente aumenta um pouco ao longo dos lags 2 a 4 , e finalmente, a rejeição da normalidade aumenta do lag 1 em relação aos demais.

Agora, avaliamos a função de autodependência local escrita em termos de cópula. O comportamento desta função teórica ao longo dos quatro primeiros lags pode ser observado na Figura 4.28 (o qual mostra-se muito similar a $\Lambda_{\tau}$ escrita em termos de função de distribuição - veja Figura 4.25). Na Figura 4.29 observamos os gráficos do seu respectivo estimador $\hat{\Lambda}_{\tau}, \tau=1,2,3,4$, os quais apresentam resultados muito similares às respectivas funções teóricas (embora com leve subestimação), e também similares aos gráficos da Figura 4.26. Na Tabela 4.12 observamos que o viés diminui ao longo da grade e também ao longo dos lags. Já ao longo dos lags, o erro quadrático médio (Tabela 4.13) apresenta redução para o primeiro ponto de grade, e valores similares para os demais pontos. Por fim, a rejeição de normalidade parece aumentar um pouco ao longo dos lags (veja Tabela 4.14). Na Figura 4.30 vemos os histogramas do estimador para o lag 1, os quais não apresentam afastamento da normalidade.

Estas simulações também foram elaboradas para três tamanhos de séries menores. Analisando os resultados das quatro simulações através das figuras D.13 a D.16 do Apêndice D, para cada lag, verificamos que aumentando-se o tamanho da série, o viés e o erro quadrático médio diminuem, e a rejeição da normalidade parece diminuir. Além disso, considerando cada tamanho de série, vemos que o viés e o erro quadrático médio geralmente diminuem ao longo dos lags, e finalmente, a não rejeição da normalidade parece similar ao longo dos lags. 

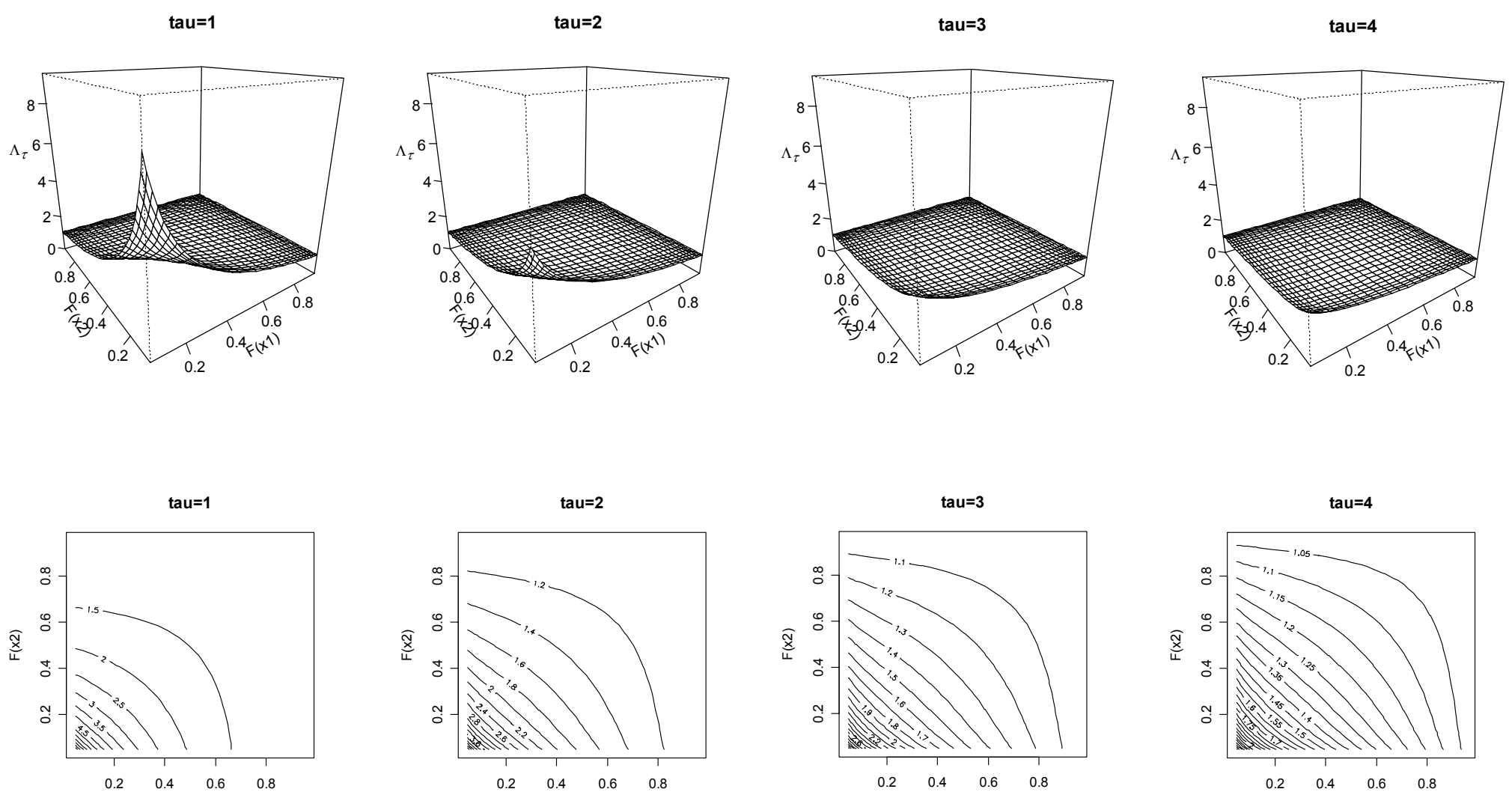

$\mathrm{F}(\mathrm{x} 1)$

$F(x 1)$

$F(x 1)$

$F(x 1)$

Figura 4.25 - Os quatro primeiros gráficos referem-se à $\Lambda_{\tau}, \tau=1,2,3,4$, via funções de distribuição, e os quatro gráficos seguintes são suas respectivas curvas de nível, considerando 200 séries com $T=1.000$ observadas de um processo Gaussiano. 

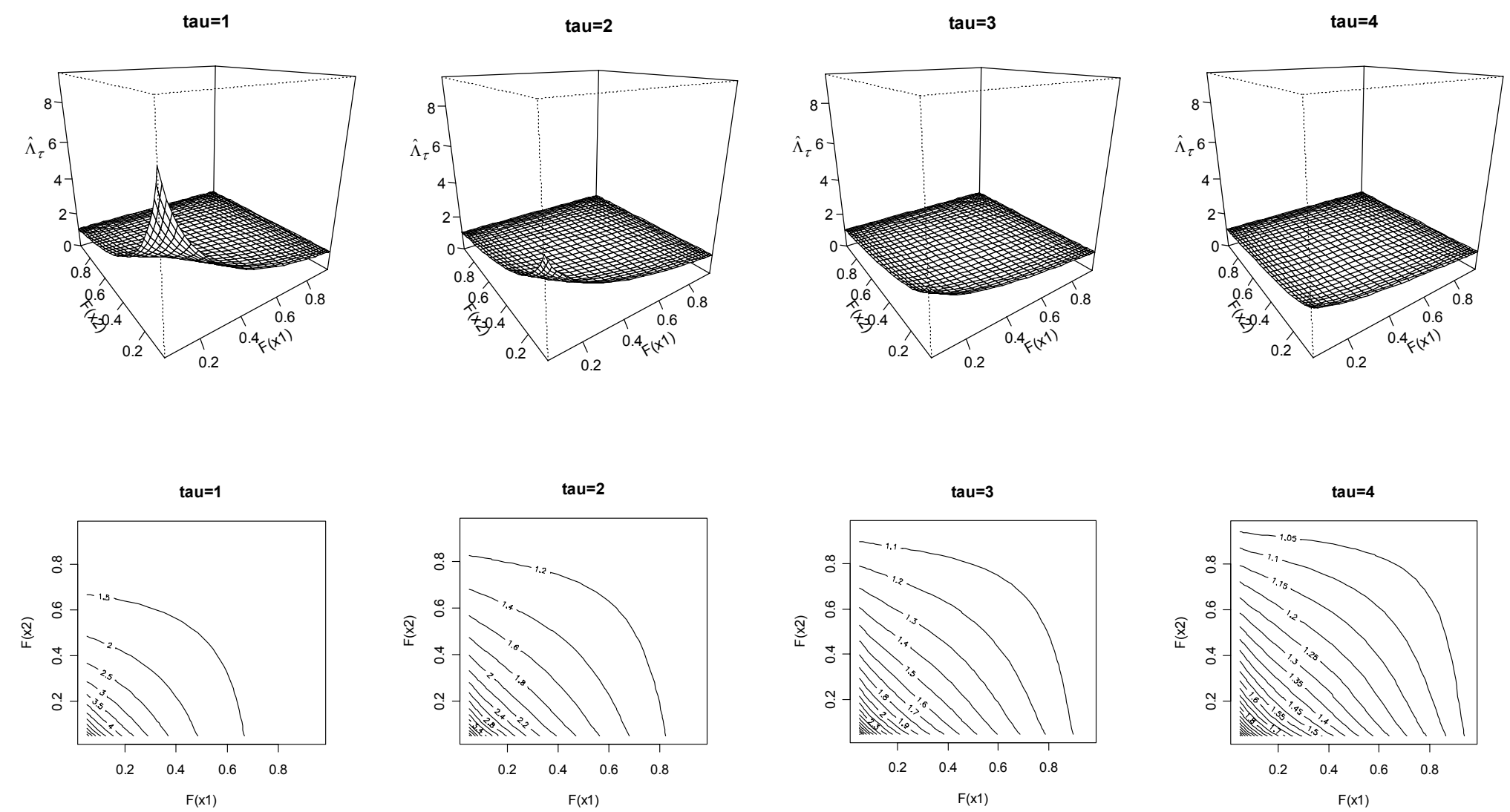

Figura 4.26 - Os quatro primeiros gráficos referem-se à $\hat{\Lambda}_{\tau}, \tau=1,2,3,4$, via funções de distribuição, e os quatro gráficos seguintes são suas respectivas curvas de nível, considerando 200 séries com $T=1.000$ observadas de um processo Gaussiano. 
Tabela 4.9 - Viés de $\hat{\Lambda}_{\tau}$ em pontos da diagonal principal da grade de $\hat{\Lambda}_{\tau}$, considerando 200 séries de tamanho 1.000 , observadas de um processo Gaussiano.

\begin{tabular}{|c|c|c|c|c|c|c|c|}
\hline Grade & $\mathbf{0 , 0 5}$ & $\mathbf{0 , 1 4}$ & $\mathbf{0 , 2 9}$ & $\mathbf{0 , 5 0}$ & $\mathbf{0 , 7 1}$ & $\mathbf{0 , 8 6}$ & \multicolumn{1}{c|}{$\mathbf{0 , 9 5}$} \\
\hline $\operatorname{Lag} 1$ & $-0,503$ & $-0,120$ & $-0,033$ & $-0,009$ & 0,000 & 0,002 & 0,001 \\
\hline $\operatorname{Lag} 2$ & $-0,301$ & $-0,080$ & $-0,023$ & $-0,004$ & 0,001 & 0,001 & 0,000 \\
\hline $\operatorname{Lag} 3$ & $-0,105$ & $-0,051$ & $-0,013$ & $-0,003$ & 0,001 & 0,001 & 0,000 \\
\hline $\operatorname{Lag} 4$ & $-0,112$ & $-0,040$ & $-0,009$ & 0,000 & 0,001 & 0,001 & 0,000 \\
\hline
\end{tabular}

Tabela 4.10 - Erro quadrático médio de $\hat{\Lambda}_{\tau}$ em pontos da diagonal principal da grade de $\hat{\Lambda}_{\tau}$, considerando 200 séries de tamanho 1.000, observadas de um processo Gaussiano.

\begin{tabular}{|c|c|c|c|c|c|c|c|}
\hline Grade & $\mathbf{0 , 0 5}$ & $\mathbf{0 , 1 4}$ & $\mathbf{0 , 2 9}$ & $\mathbf{0 , 5 0}$ & $\mathbf{0 , 7 1}$ & $\mathbf{0 , 8 6}$ & $\mathbf{0 , 9 5}$ \\
\hline Lag 1 & 2,200 & 0,158 & 0,023 & 0,004 & 0,001 & 0,000 & 0,000 \\
\hline $\operatorname{Lag} 2$ & 1,389 & 0,103 & 0,015 & 0,002 & 0,001 & 0,000 & 0,000 \\
\hline $\operatorname{Lag} 3$ & 1,101 & 0,088 & 0,012 & 0,002 & 0,000 & 0,000 & 0,000 \\
\hline $\operatorname{Lag} 4$ & 0,861 & 0,088 & 0,011 & 0,002 & 0,000 & 0,000 & 0,000 \\
\hline
\end{tabular}

Tabela 4.11 - $P$-valor do teste de normalidade de Jarque Bera para $\hat{\Lambda}_{\tau}$ em pontos da diagonal principal da grade de $\hat{\Lambda}_{\tau}$, considerando 200 séries de tamanho 1.000, observadas de um processo Gaussiano.

\begin{tabular}{|c|c|c|c|c|c|c|c|}
\hline Grade & $\mathbf{0 , 0 5}$ & $\mathbf{0 , 1 4}$ & $\mathbf{0 , 2 9}$ & $\mathbf{0 , 5 0}$ & $\mathbf{0 , 7 1}$ & $\mathbf{0 , 8 6}$ & \multicolumn{1}{c|}{$\mathbf{0 5}$} \\
\hline $\operatorname{Lag} 1$ & 0,000 & 0,473 & 0,207 & 0,338 & 0,129 & 0,216 & 0,141 \\
\hline $\operatorname{Lag} 2$ & 0,000 & 0,042 & 0,012 & 0,705 & 0,405 & 0,009 & 0,001 \\
\hline $\operatorname{Lag} 3$ & 0,000 & 0,097 & 0,000 & 0,099 & 0,648 & 0,001 & 0,000 \\
\hline $\operatorname{Lag} 4$ & 0,001 & 0,072 & 0,005 & 0,083 & 0,126 & 0,006 & 0,000 \\
\hline
\end{tabular}


0.05

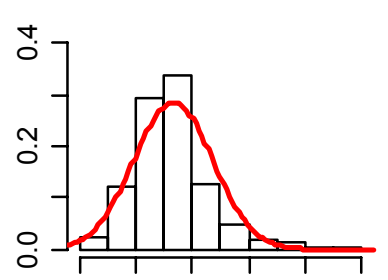

$\begin{array}{llllll}4 & 6 & 8 & 10 & 12 & 14\end{array}$

0.71

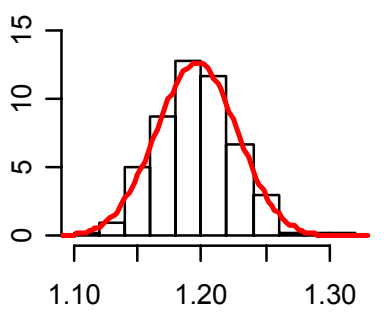

0.14

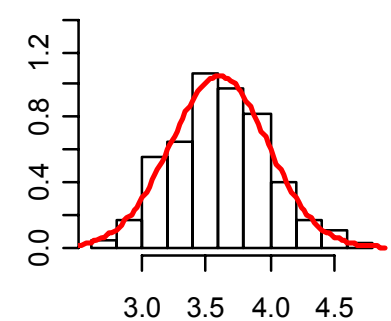

0.86

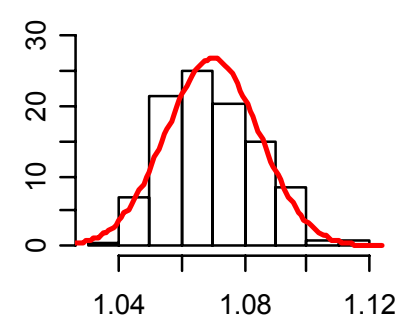

0.29

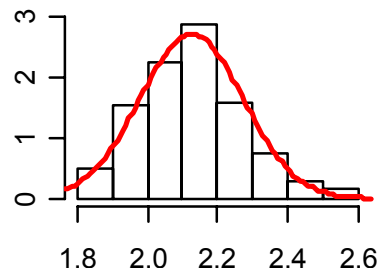

0.95

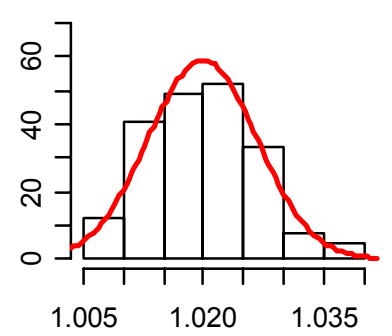

Figura 4.27 - Histogramas das estimações de $\hat{\Lambda}_{\tau}$ no lag 1 em pontos da diagonal principal da grade, considerando 200 séries de tamanho 1.000 observadas de um processo Gaussiano.

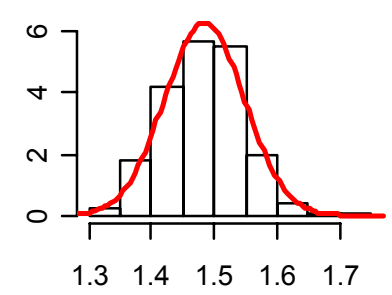

$1.3 \quad 1.4 \quad 1.5 \quad 1.6 \quad 1.7$$$
\text { . } 200 \text { séries de tamanho } 1.000 \text { observadas de um processo Gaussiano. }
$$ 

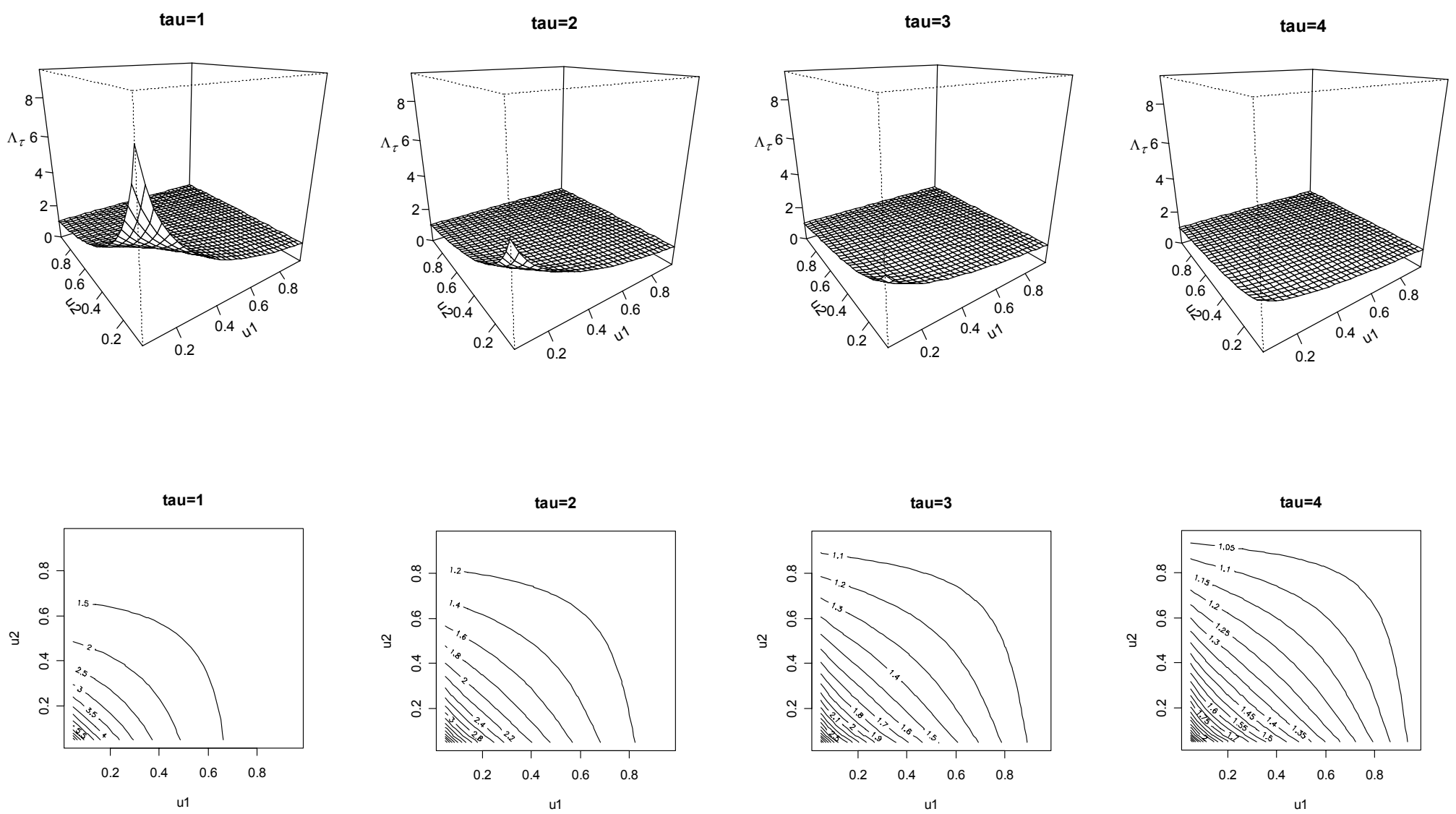

Figura 4.28 - Os quatro primeiros gráficos referem-se à $\Lambda_{\tau}, \tau=1,2,3,4$, via cópula, e os quatro gráficos seguintes são suas respectivas curvas de nível, considerando 200 séries com $T=500$ observadas de um processo Gaussiano. 
tau $=1$

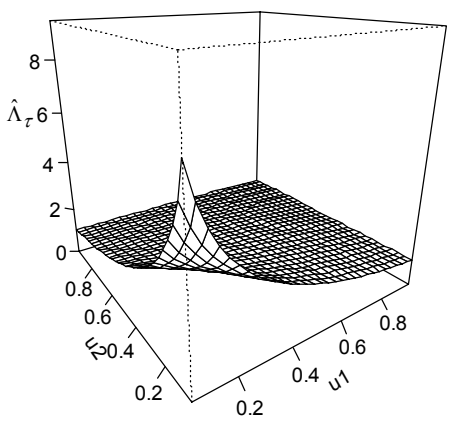

tau $=1$

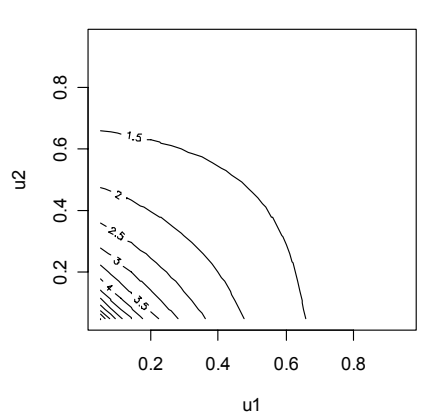

tau=2

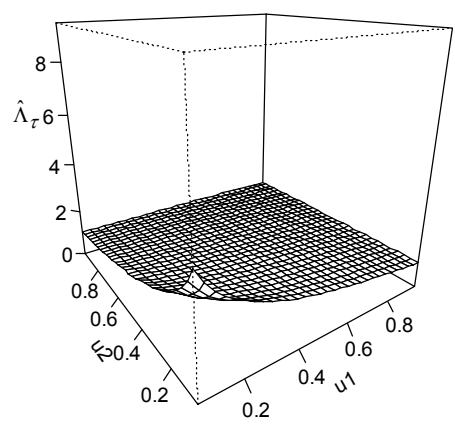

tau $=2$

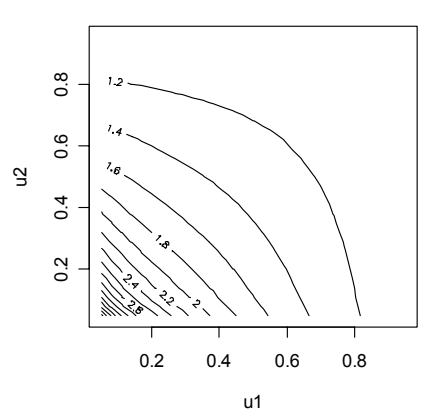

tau $=3$

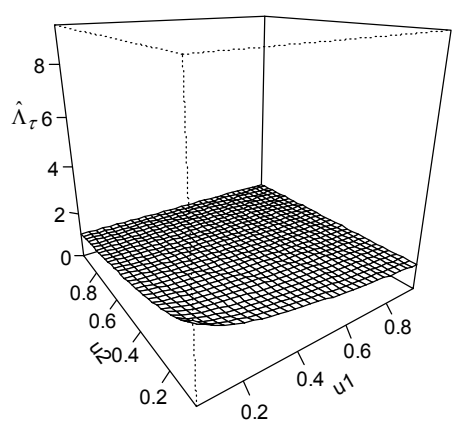

tau=3

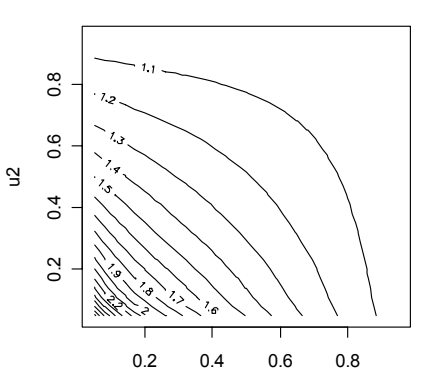

u1 tau $=4$
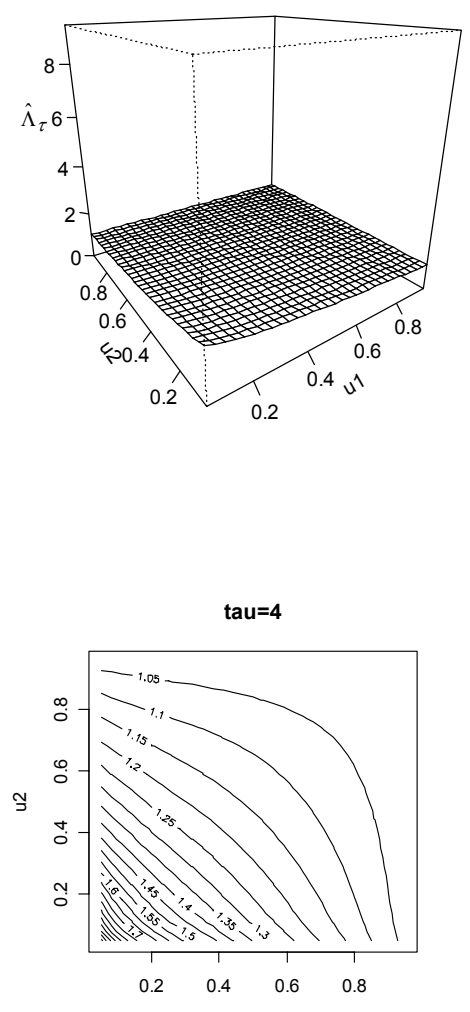

u1

Figura 4.29 - Os quatro primeiros gráficos referem-se à $\hat{\Lambda}_{\tau}, \tau=1,2,3,4$, via cópula, e os quatro gráficos seguintes são suas respectivas curvas de nível, considerando 200 séries com $T=500$ observadas de um processo Gaussiano. 
Tabela 4.12 - Viés de $\hat{\Lambda}_{\tau}$ em pontos da diagonal principal da grade de $\hat{\Lambda}_{\tau}$, considerando 200 séries de tamanho 500, observadas de um processo Gaussiano.

\begin{tabular}{|c|l|l|l|l|l|l|l|}
\hline Grade & $\mathbf{0 , 0 5}$ & $\mathbf{0 , 2 0}$ & $\mathbf{0 , 3 5}$ & $\mathbf{0 , 5 0}$ & $\mathbf{0 , 6 5}$ & $\mathbf{0 , 8 0}$ & $\mathbf{0 , 9 5}$ \\
\hline Lag 1 & $-0,788$ & $-0,129$ & $-0,060$ & $-0,030$ & $-0,014$ & $-0,007$ & $-0,002$ \\
\hline Lag 2 & $-0,497$ & $-0,093$ & $-0,038$ & $-0,019$ & $-0,009$ & $-0,005$ & $-0,002$ \\
\hline $\operatorname{Lag} 3$ & $-0,408$ & $-0,064$ & $-0,032$ & $-0,018$ & $-0,008$ & $-0,004$ & $-0,001$ \\
\hline Lag 4 & $-0,313$ & $-0,053$ & $-0,025$ & $-0,014$ & $-0,006$ & $-0,004$ & $-0,001$ \\
\hline
\end{tabular}

Tabela 4.13 - Erro quadrático médio de $\hat{\Lambda}_{\tau}$ em pontos da diagonal principal da grade de $\hat{\Lambda}_{\tau}$, considerando 200 séries de tamanho 500, observadas de um processo Gaussiano.

\begin{tabular}{|c|c|c|c|c|c|c|c|}
\hline Grade & 0,05 & 0,20 & 0,35 & 0,50 & 0,65 & 0,80 & 0,95 \\
\hline Lag 1 & 2,993 & 0,051 & 0,009 & 0,002 & 0,001 & 0,000 & 0,000 \\
\hline $\operatorname{Lag} 2$ & 2,485 & 0,052 & 0,009 & 0,002 & 0,001 & 0,000 & 0,000 \\
\hline $\operatorname{Lag} 3$ & 2,253 & 0,052 & 0,010 & 0,003 & 0,001 & 0,000 & 0,000 \\
\hline $\operatorname{Lag} 4$ & 1,858 & 0,053 & 0,010 & 0,003 & 0,001 & 0,000 & 0,000 \\
\hline
\end{tabular}

Tabela $4.14-P$-valor do teste de normalidade de Jarque Bera para $\hat{\Lambda}_{\tau}$ em pontos da diagonal principal da grade de $\hat{\Lambda}_{\tau}$, considerando 200 séries de tamanho 500, observadas de um processo Gaussiano.

\begin{tabular}{|c|c|c|c|c|c|c|c|}
\hline Grade & $\mathbf{0 , 0 5}$ & $\mathbf{0 , 2 0}$ & $\mathbf{0 , 3 5}$ & $\mathbf{0 , 5 0}$ & $\mathbf{0 , 6 5}$ & $\mathbf{0 , 8 0}$ & \multicolumn{1}{c|}{$\mathbf{0 , 9 5}$} \\
\hline Lag 1 & 0,172 & 0,737 & 0,059 & 0,588 & 0,884 & 0,280 & 0,985 \\
\hline Lag 2 & 0,005 & 0,399 & 0,745 & 0,967 & 0,707 & 0,776 & 0,484 \\
\hline Lag 3 & 0,000 & 0,346 & 0,576 & 0,516 & 0,607 & 0,768 & 0,000 \\
\hline Lag 4 & 0,000 & 0,470 & 0,643 & 0,983 & 0,808 & 0,720 & 0,000 \\
\hline
\end{tabular}



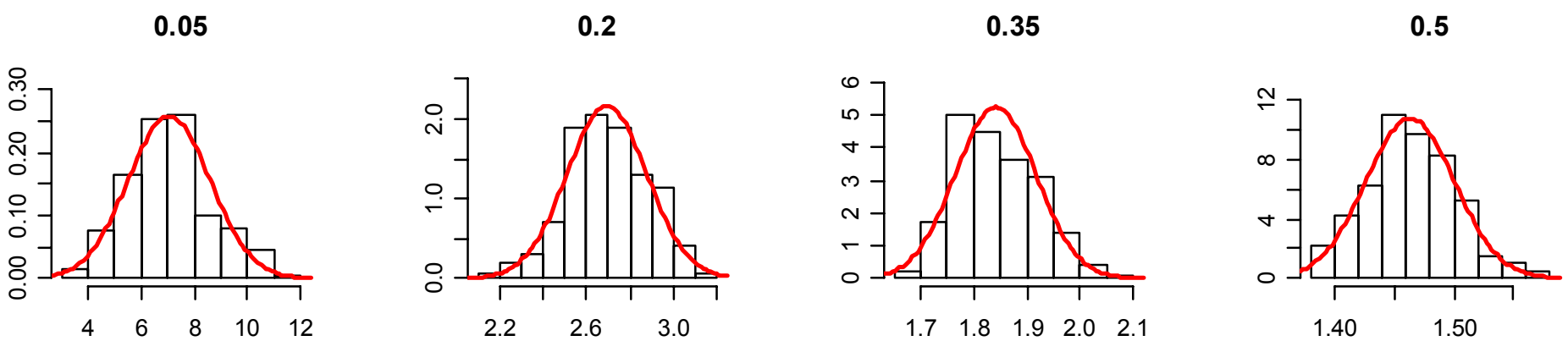

0.65

0.8

0.95
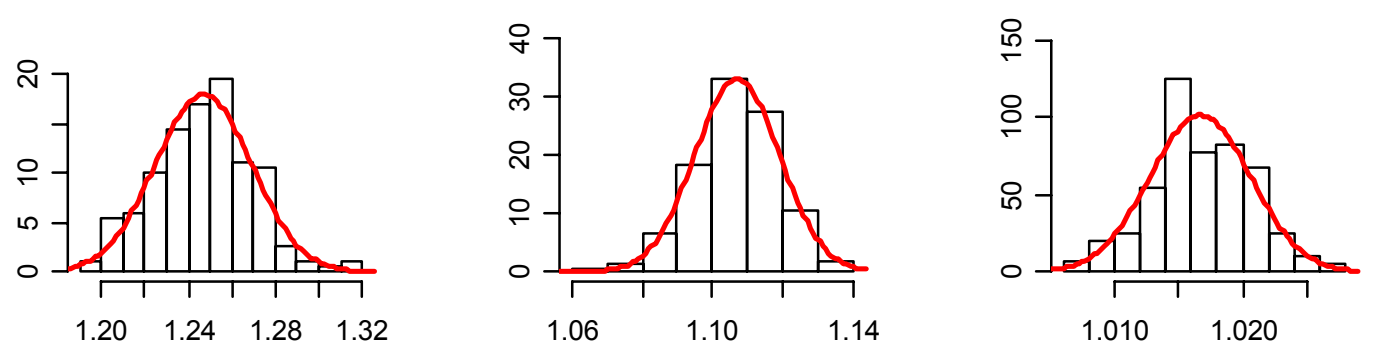

Figura 4.30 - Histogramas das estimações de $\hat{\Lambda}_{\tau}$ no lag 1 em pontos da diagonal principal da grade, considerando 200 séries de tamanho 500 observadas de um processo Gaussiano. 


\subsubsection{Aplicações a séries reais}

A seguir, estimamos a função de autodependência local nos quatro primeiros lags considerando novamente os retornos diários da Petrobrás e do Banespa, de 3/jan/95 a 27/dez/00. A estimação foi elaborada de forma similar à seção anterior, mas agora utilizando $90 \%$ dos dados centrais.

Primeiramente, a função de autodependência local é simulada utilizando o estimador (4.10).

A Figura 4.31 apresenta $\hat{\Lambda}_{\tau}$ e seus respectivos gráficos de curvas de nível para as quatro primeiras defasagens dos retornos diários da Petrobrás. Vemos que a dependência positiva apresenta um decaimento suave, lembrando um processo autoregressivo. Além disso, essa dependência pode ser observada em grande parte da extensão do primeiro quadrante no lag 1, com uma redução considerável ao longo dos lags. Para as mesmas defasagens, na Figura 4.32 observamos as cópulas empíricas suavizadas e suas respectivas curvas de nível, as quais indicam maior dependência positiva no lag 1. Esses resultados são similares aos obtidos na seção 2.1.7, em que a f.a.c. dos retornos ao quadrado (Figura 2.19) indica clara dependência positiva, a f.a.c.l. indica dependência positiva no lag 1 (Figura 2.20), assim como informa os gráficos dos postos normalizados (Figura 2.21).

Os retornos diários do Banespa apresentam uma destacada dependência positiva no lag 1, e pequenas e similares dependência positivas nos três lags seguintes (veja Figura 4.33). Este comportamento lembra um processo de médias móveis. Observamos que essa dependência é localizada apenas no início do primeiro quadrante. Na Figura 4.34 apresentam-se as cópulas empíricas suavizadas e suas correspondentes curvas de nível, que parece indicar alguma dependência positiva no lag 1. Confrontando esses resultados com os obtidos na seção 2.1.7, vemos concordância entre os mesmos.

Agora, para as mesmas séries da Petrobrás e do Banespa, $\hat{\Lambda}_{\tau}, \tau=1,2,3,4$, são estimadas com base no estimador (4.11), resultando nos gráficos das figuras 4.35 e 4.36, respectivamente. Comparando estes gráficos com os gráficos das figuras 4.31 e 4.33, vemos que o estimador via cópula apresenta resultados muito similares ao estimador via funções de distribuição. 

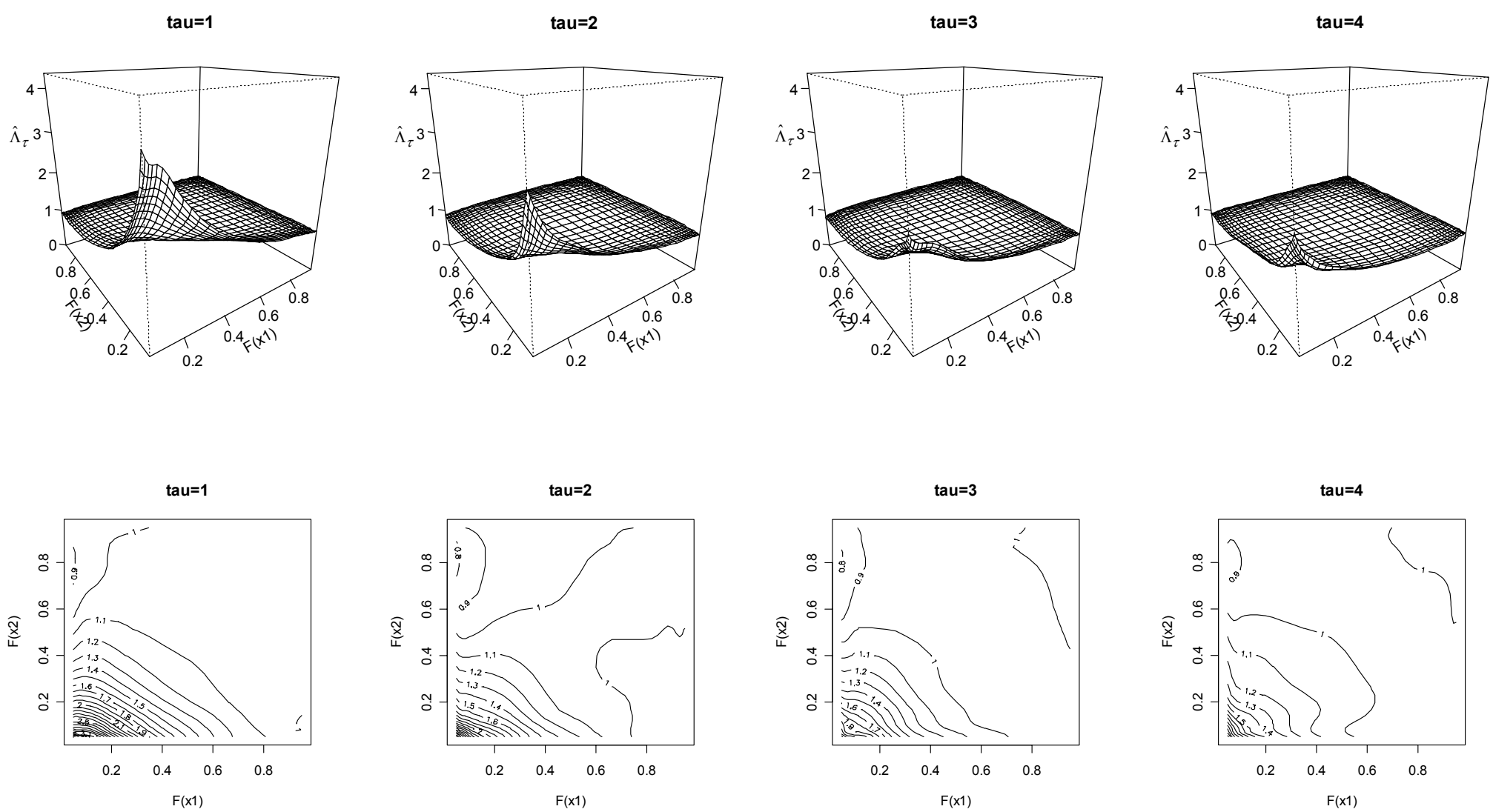

Figura 4.31 - Função de autodependência local estimada via funções de distribuição para os retornos diários da Petrobrás (período de 3/jan/95 a 27/dez/00), e respectivos gráficos de curvas de nível para os lags de 1 a 4. 

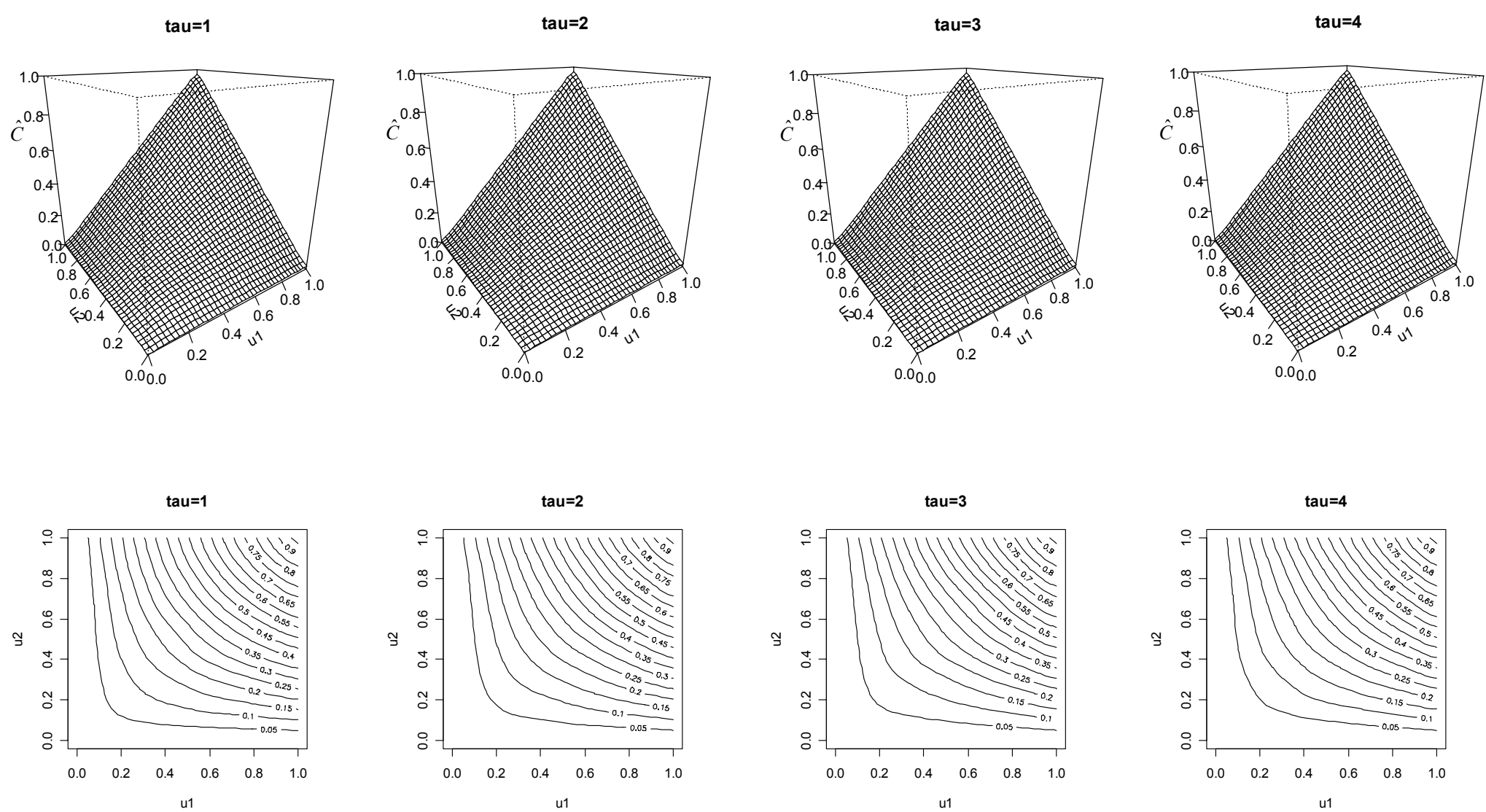

Figura 4.32 - Cópula empírica suavizada estimada e respectivos gráficos de curvas de nível nos lags 1 a 4 para dos retornos diários da Petrobrás (período de 3/jan/95 a 27/dez/00). 
tau $=$

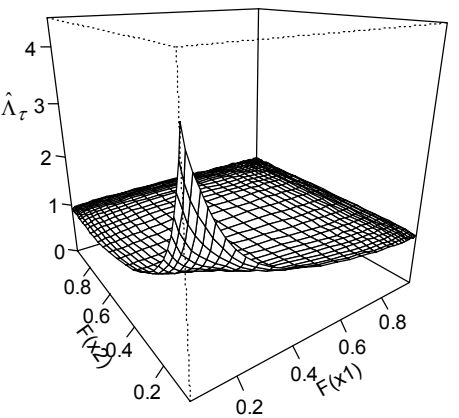

tau $=1$

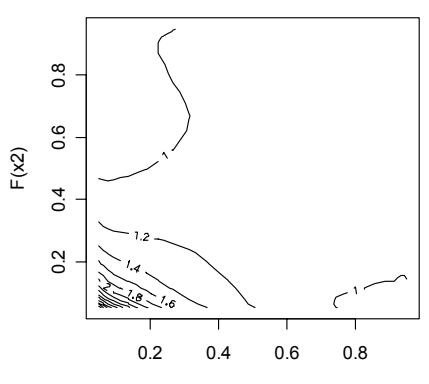

$\mathrm{F}(\mathrm{x} 1)$ tau $=2$

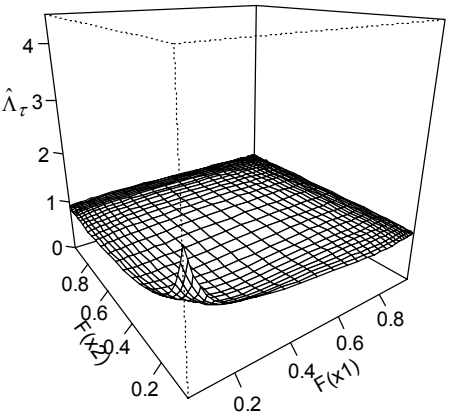

$\mathrm{au}=2$

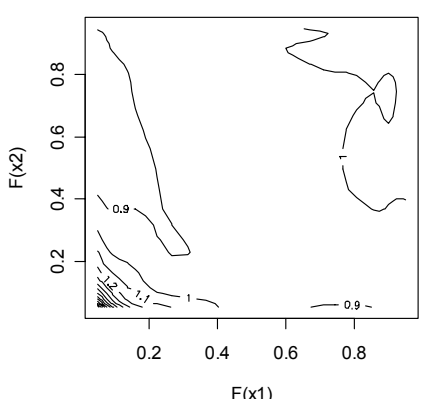

tau=3

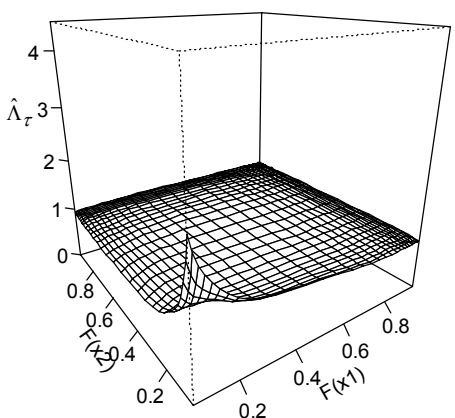

tau=3

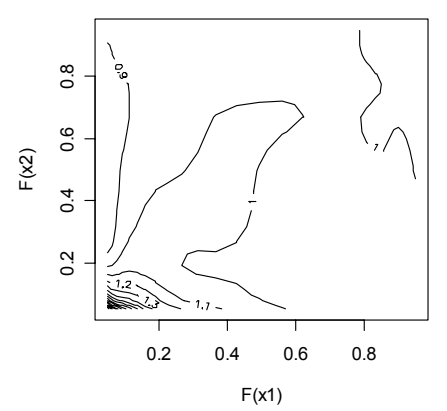

tau $=4$
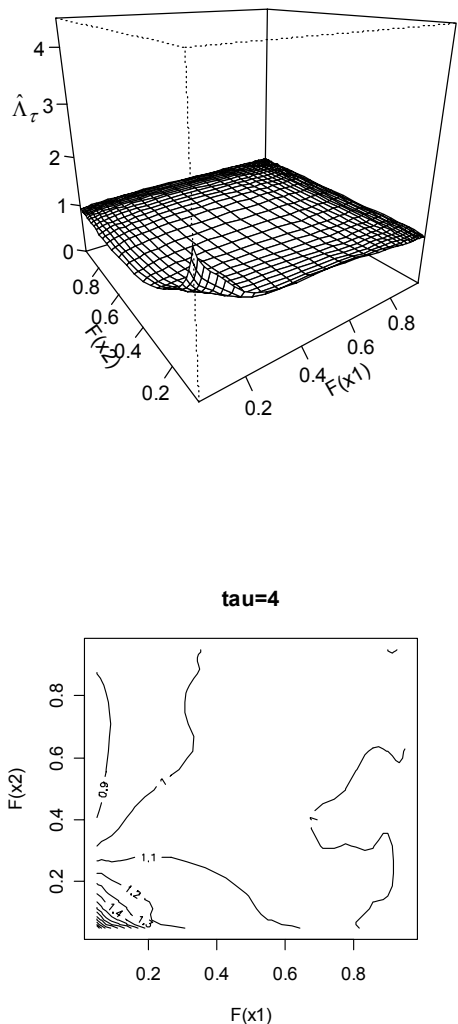

Figura 4.33 - Função de autodependência local estimada via funções de distribuição para os retornos diários do Banespa (período de 3/jan/95 a 27/dez/00), e respectivos gráficos de curvas de nível para os lags de 1 a 4. 

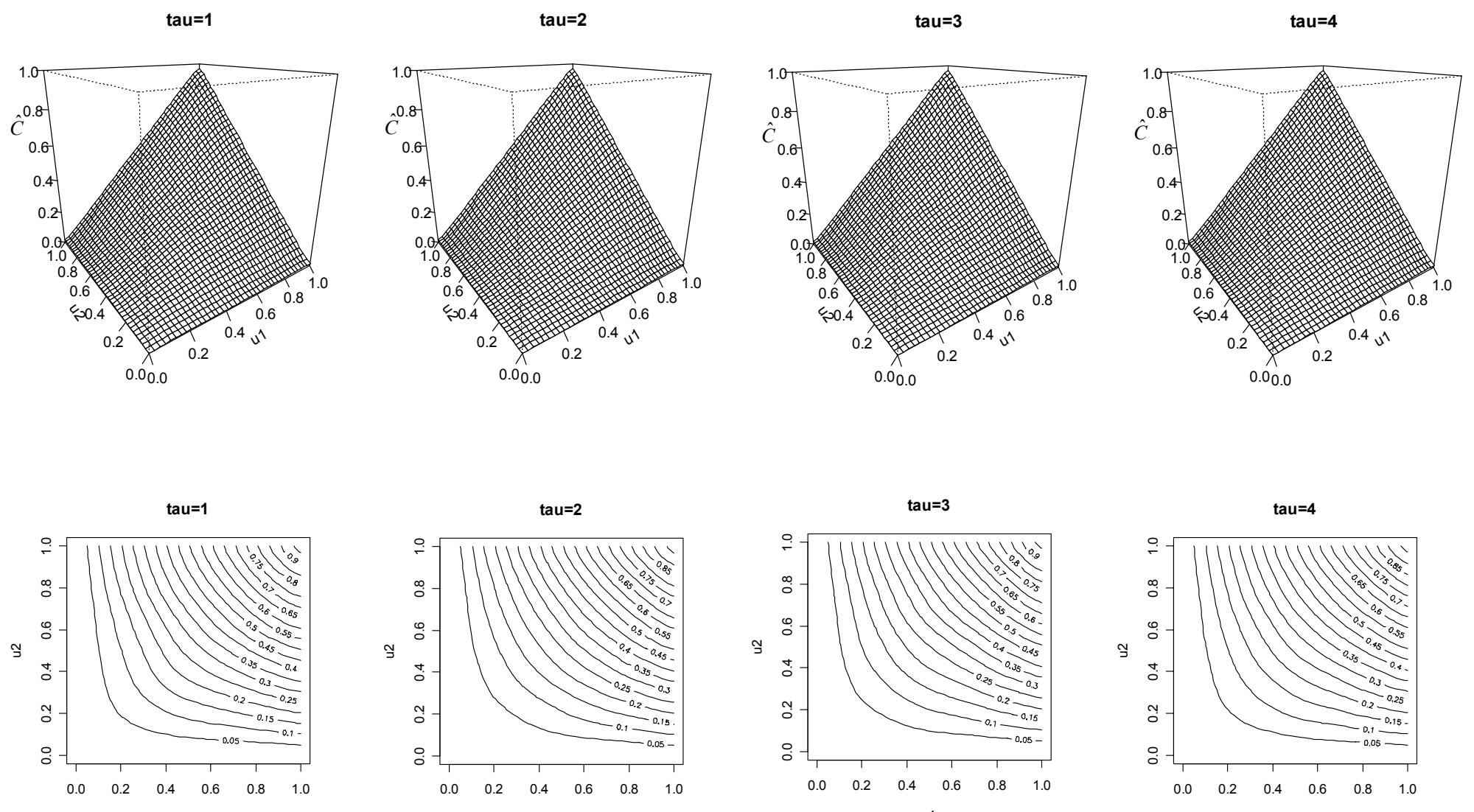

u1

Figura 4.34 - Cópula empírica suavizada estimada e respectivos gráficos de curvas de nível nos lags 1 a 4 para os retornos diários do Banespa (período de 3/jan/95 a 27/dez/00). 
tau $=1$
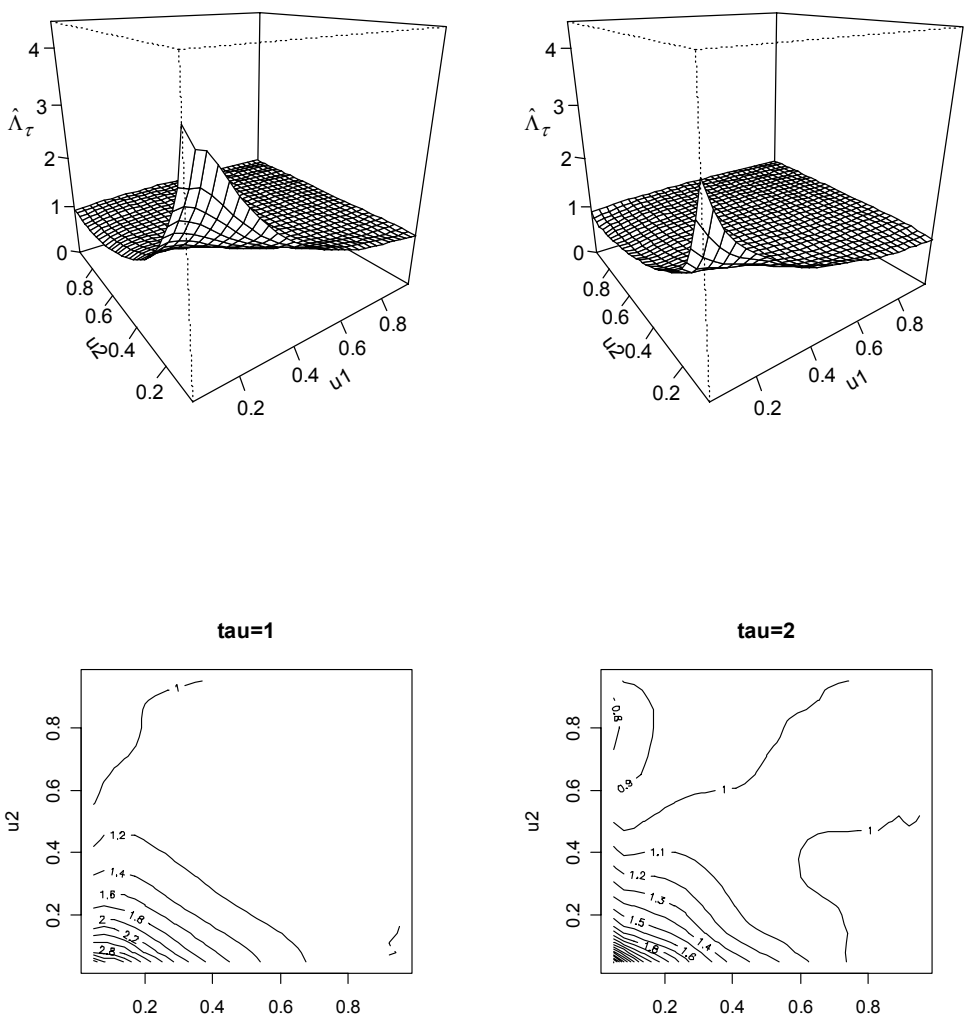

u1

tau=2

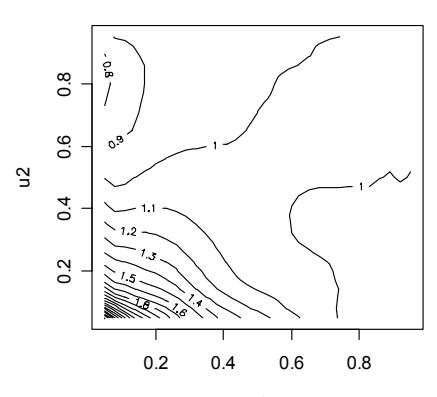

u1 tau=2

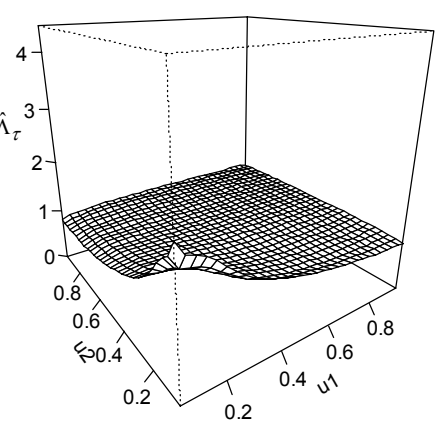

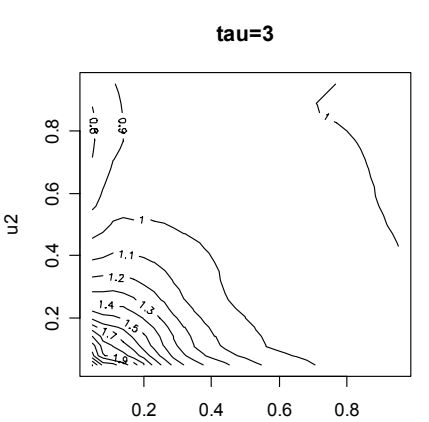

tau=4
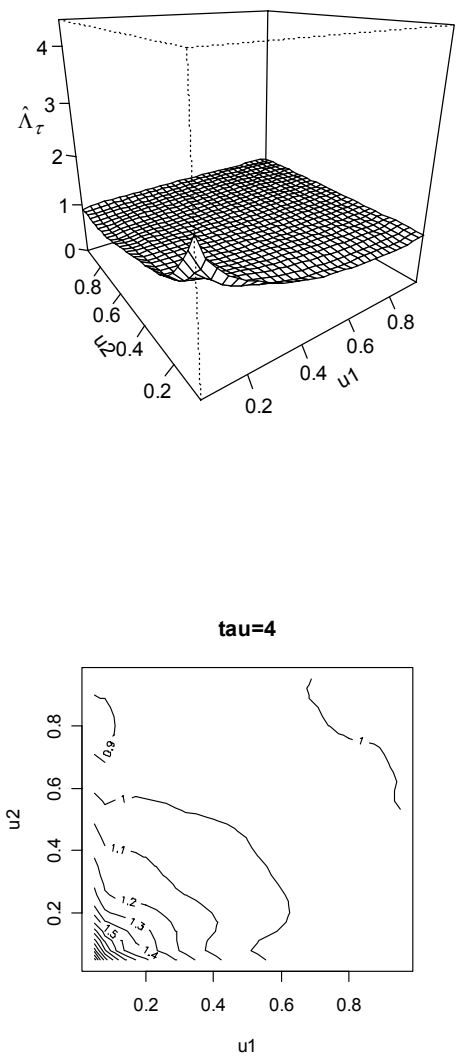

Figura 4.35 - Função de autodependência local estimada via cópula para os retornos diários da Petrobrás (período de 3/jan/95 a 27/dez/00), e respectivos gráficos de curvas de nível para os lags de 1 a 4. 
tau $=1$
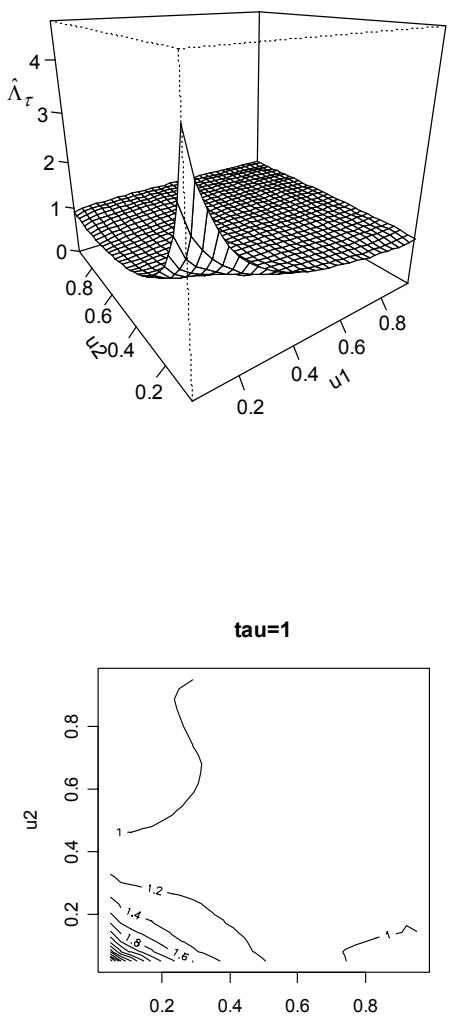

u1 tau $=2$
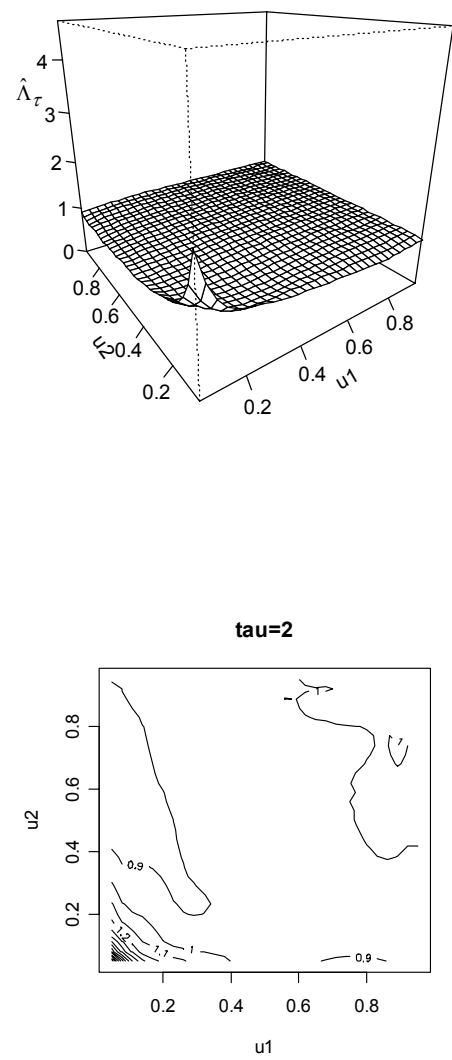

tau $=3$

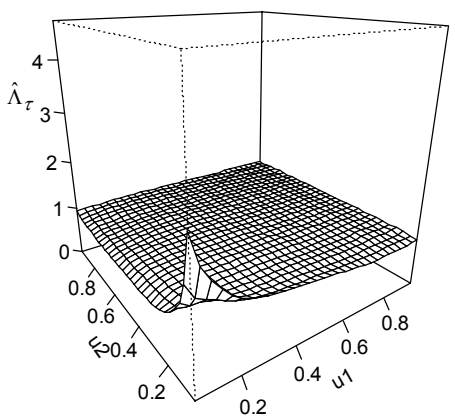

0.2

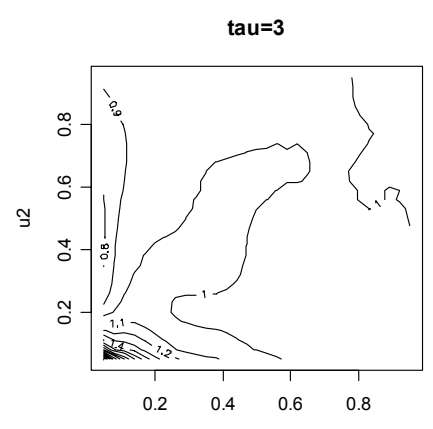

u1 tau=4
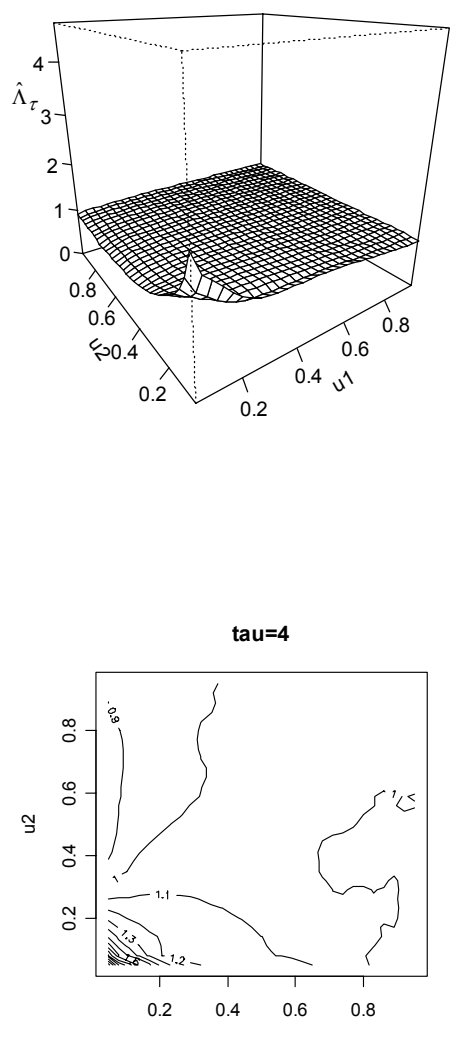

u1

Figura 4.36 - Função de autodependência local estimada via cópula para os retornos diários do Banespa

(período de 3/jan/95 a 27/dez/00) e respectivos gráficos de curvas de nível para os lags de 1 a 4. 


\section{Capítulo 5}

\section{Conclusões}

Neste trabalho consideramos três medidas de dependência local e suas extensões para séries temporais estacionárias. Para a medida de Sibuya propomos também estimadores para o caso de variáveis aleatórias.

Para as três medidas locais estudadas, verificamos a consistência dos estimadores teoricamente e também através de simulações. Também, observamos a indicação de normalidade dos estimadores (exceto para a curva de correlação cruzada contemporânea e a curva de autocorrelação, possivelmente devido a necessidade de amostras maiores).

Como possíveis linhas de pesquisa futuras, destaco:

1. obter as distribuições assintóticas dos estimadores propostos;

2. usar estas distribuições para obter testes de independência;

3. considerar formas alternativas da medida $H$;

4. considerar medidas de simetria para variáveis aleatórias e respectivos testes;

5. se a possibilidade em (1) não se concretizar, considerar a avaliação dos estimadores via bootstrap;

6. avaliar mais profundamente as relações dessas medidas com cópulas, quando possível;

7. utilizar outros estimadores não-paramétricos para $H, \rho$ e $\Lambda$, usando splines ou ondaletas. 


\section{Apêndice A - Demonstrações}

\section{Capítulo 3 - Curva de Autocorrelação}

\section{Modelo AR(1)}

Para obtermos a expressão de $\rho_{\tau}(x)$, precisamos de $E\left[X_{t+\tau} / X_{t}=x\right], \beta_{\tau}(x)$ e $\operatorname{Var}\left[X_{t+\tau} / X_{t}=x\right]$.

A $E\left[X_{t+\tau} / X_{t}=x\right],|\tau| \geq 1$, é dada por (2.4), e para $\tau=0, E\left[X_{t} / X_{t}=x\right]=x$, ou seja

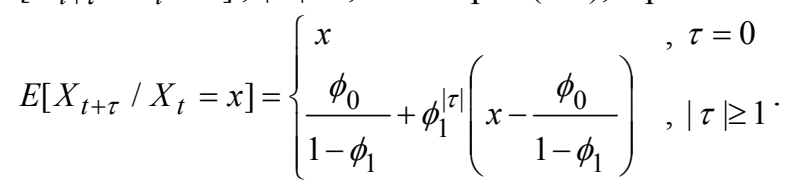

Derivando esta esperança condicional em relação a $x$, obtemos o coeficiente angulat local $\beta_{\tau}(x)$, isto é

$$
\beta_{\tau}(x)=\frac{\partial}{\partial x} E\left[X_{t+\tau} / X_{t}=x\right]=\left\{\begin{array}{cc}
1 & , \tau=0 \\
\phi_{1}^{|\tau|} & ,|\tau| \geq 1
\end{array},\right.
$$

ou seja,

$$
\beta_{\tau}(x)=\left\{\begin{array}{cl}
1 & , \tau=0 \\
\phi_{1}^{|\tau|} & ,|\tau| \geq 1
\end{array} .\right.
$$

É fácil ver que

$$
\operatorname{Var}\left[X_{t+\tau} / X_{t}=x\right]= \begin{cases}0 & , \tau=0 \\ \sigma_{\varepsilon}^{2} \sum_{k=0}^{\tau-1} \phi_{1}^{2 k} & , \tau \geq 1\end{cases}
$$

ou então

$$
\operatorname{Var}\left[X_{t+\tau} / X_{t}=x\right]=\left\{\begin{array}{ll}
0 & , \tau=0 \\
\frac{\sigma_{\varepsilon}^{2}}{1-\phi_{1}^{2}}\left(1-\phi_{1}^{2 \tau}\right) & , \tau \geq 1
\end{array} .\right.
$$

Para $\tau \leq-1$, obtemos a mesma expressão, e então

$$
\operatorname{Var}\left[X_{t+\tau} / X_{t}=x\right]=\left\{\begin{array}{ll}
0 & , \tau=0 \\
\frac{\sigma_{\varepsilon}^{2}}{1-\phi_{1}^{2}}\left(1-\phi_{1}^{2|\tau|}\right) & ,|\tau| \geq 1
\end{array} .\right.
$$

Como para o modelo em questão temos

$$
\mu=\frac{\phi_{0}}{1-\phi_{1}}, \sigma^{2}=\frac{\sigma_{\varepsilon}^{2}}{1-\phi_{1}^{2}}=\gamma_{0}, \rho_{\tau}=\phi_{1}^{\tau} \text { para } \tau \geq 0, \text { sendo } \rho_{\tau}=\rho_{-\tau},
$$

então a variância condicional acima possui a seguinte representação alternativa:

$$
\operatorname{Var}\left[X_{t+\tau} / X_{t}=x\right]=\gamma_{0}\left(1-\phi_{1}^{2|\tau|}\right), \forall x \in S, \forall \tau \in Z \text {. }
$$

Portanto, substituindo as expressões do coeficiente angular local e da variância condicional local na expressão geral de $\rho_{\tau}(x), \forall x \in S, \forall \tau \in Z$, dada por (3.5), temos que: 


$$
\rho_{\tau}(x)=\frac{\beta_{\tau}(x) \sqrt{\gamma_{0}}}{\sqrt{\beta_{\tau}^{2}(x) \gamma_{0}+\sigma_{\tau}^{2}(x)}}= \begin{cases}1 & , \tau=0 \\ \frac{\phi_{1}^{|\tau|} \sqrt{\gamma_{0}}}{\sqrt{\phi_{1}^{2|\tau|} \gamma_{0}+\gamma_{0}\left(1-\phi_{1}^{2|\tau|}\right)}} & ,|\tau| \geq 1,\end{cases}
$$

ou seja

$$
\rho_{\tau}(x)=\rho_{\tau}, \forall x \in S, \tau \in Z .
$$

\section{Modelo MA(1)}

Para obtermos as expressões de $E\left[X_{t+\tau} / X_{t}=x\right], \beta_{\tau}(x)$ e $\operatorname{Var}\left[X_{t+\tau} / X_{t}=x\right]$, e então calcularmos $\rho_{\tau}(x)$, vamos considerar o modelo $\operatorname{MA}(1)$ escrito como um $\operatorname{AR}(\infty)$, ou seja

$$
X_{t}=\theta_{0} /\left(1-\theta_{1}\right)-\sum_{j=1}^{\infty} \theta_{1}^{j} X_{t-j}+a_{t}, a_{t} \sim \operatorname{iid}\left(0, \sigma_{\varepsilon}^{2}\right) .
$$

Em (2.7) obtivemos a expressão de $E\left[X_{t+\tau} / X_{t}=x\right]$ para $|\tau| \geq 1$, e como para $\tau=0$ esta esperança condicional resulta em $x$, então

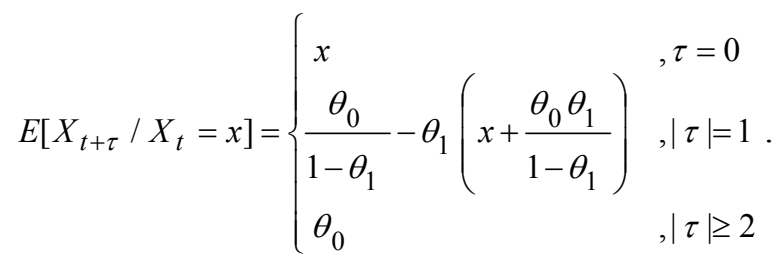

Portanto, $\beta_{\tau}(x)$ resulta em

$$
\beta_{\tau}(x)=\frac{\partial}{\partial x} E\left[X_{t+\tau} / X_{t}=x\right]= \begin{cases}1 & , \tau=0 \\ -\theta_{1} & ,|\tau|=1 \\ 0 & ,|\tau| \geq 2\end{cases}
$$

A seguir, vamos calcular $\operatorname{Var}\left[X_{t+\tau} / X_{t}=x\right], \forall x \in S, \forall t \in Z$, para $\tau=0$ e $\tau=1$ (pois para $|\tau| \geq 2$, $\beta_{\tau}(x)=0$ e então $\left.\rho_{\tau}(x)=0\right)$.

- $\tau=0$

$\operatorname{Var}\left[X_{t} / X_{t}=x\right]=0$,

- $\tau=1$

$$
\begin{aligned}
& \operatorname{Var}\left[X_{t+1} / X_{t}=x\right]=\operatorname{Var}\left[\theta_{0} /\left(1-\theta_{1}\right)-\theta_{1} X_{t}-\theta_{1}^{2} X_{t-1}-\theta_{1}^{3} X_{t-2}-\theta_{1}^{4} X_{t-3}-\ldots+a_{t+1} / X_{t}=x\right] \\
& =\theta_{1}^{4} \operatorname{Var}\left[X_{t-1} / X_{t}=x\right]+\theta_{1}^{6} \operatorname{Var}\left[X_{t-2} / X_{t}=x\right]+\theta_{1}^{8} \operatorname{Var}\left[X_{t-3} / X_{t}=x\right]+\ldots+\operatorname{Var}\left[a_{t+1} / X_{t}=x\right] \\
& +2 \theta_{1}^{5} \operatorname{Cov}\left(\left(X_{t-1}, X_{t-2}\right) / X_{t}=x\right)+2 \theta_{1}^{6} \operatorname{Cov}\left(\left(X_{t-1}, X_{t-3}\right) / X_{t}=x\right)+\ldots \\
& +2 \theta_{1}^{7} \operatorname{Cov}\left(\left(X_{t-2}, X_{t-3}\right) / X_{t}=x\right)+2 \theta_{1}^{8} \operatorname{Cov}\left(\left(X_{t-2}, X_{t-4}\right) / X_{t}=x\right)+\ldots \\
& +2 \theta_{1}^{9} \operatorname{Cov}\left(\left(X_{t-3}, X_{t-4}\right) / X_{t}=x\right)+2 \theta_{1}^{10} \operatorname{Cov}\left(\left(X_{t-3}, X_{t-5}\right) / X_{t}=x\right)+\ldots \\
& +\ldots \\
& -2 \theta_{1}^{2} \operatorname{Cov}\left(\left(X_{t-1}, a_{t+1}\right) / X_{t}=x\right)-2 \theta_{1}^{3} \operatorname{Cov}\left(\left(X_{t-2}, a_{t+1}\right) / X_{t}=x\right)-\ldots \\
& =\gamma_{0}\left(\theta_{1}^{4}+\theta_{1}^{6}+\theta_{1}^{8}+\ldots\right)+2 \gamma_{1}\left(\theta_{1}^{5}+\theta_{1}^{7}+\theta_{1}^{9}+\ldots\right)+\sigma_{a}^{2} \\
& =\gamma_{0} \frac{\theta_{1}^{4}}{1-\theta_{1}^{2}}+2 \gamma_{1} \frac{\theta_{1}^{5}}{1-\theta_{1}^{2}}+\sigma_{a}^{2}
\end{aligned}
$$




$$
=\frac{\theta_{1}^{4}}{1-\theta_{1}^{2}}\left(\gamma_{0}+2 \gamma_{1} \theta_{1}\right)+\sigma_{a}^{2},
$$

pois $X_{t-j}, j \geq 1$ não depende de $X_{t}, X_{t}$ depende de $a_{t-j}$ para $j \geq 0$, e $\gamma_{j}=0$ para $j \geq 2$.

Como $\gamma_{0}=\sigma_{a}^{2}\left(1+\theta_{1}^{2}\right)$ e $\rho_{1}=\frac{\gamma_{1}}{\gamma_{0}}=\frac{-\theta_{1}}{1+\theta_{1}^{2}} \Rightarrow \gamma_{1}=\frac{-\theta_{1} \gamma_{0}}{1+\theta_{1}^{2}}$, então

$$
\begin{aligned}
\operatorname{Var}\left[X_{t+1} / X_{t}=x\right] & =\frac{\theta_{1}^{4}}{1-\theta_{1}^{2}}\left(\sigma_{a}^{2}\left(1+\theta_{1}^{2}\right)+2 \frac{-\theta_{1} \gamma_{0}}{1+\theta_{1}^{2}} \theta_{1}\right)+\sigma_{a}^{2} \\
& =\frac{\theta_{1}^{4}}{1-\theta_{1}^{2}}\left(\sigma_{a}^{2}\left(1+\theta_{1}^{2}\right)-\frac{2 \theta_{1}^{2}}{\left(1+\theta_{1}^{2}\right)} \sigma_{a}^{2}\left(1+\theta_{1}^{2}\right)\right)+\sigma_{a}^{2} \\
& =\frac{\theta_{1}^{4}}{1-\theta_{1}^{2}}\left(\sigma_{a}^{2}-\theta_{1}^{2} \sigma_{a}^{2}\right)+\sigma_{a}^{2} \\
& =\sigma_{a}^{2}\left(1+\theta_{1}^{4}\right) .
\end{aligned}
$$

Portanto,

$$
\operatorname{Var}\left[X_{t+\tau} / X_{t}=x\right]=\left\{\begin{array}{ll}
0 & , \tau=0 \\
\sigma_{a}^{2}\left(1+\theta_{1}^{4}\right) & ,|\tau|=1
\end{array},\right.
$$

sendo que o módulo em $\tau$ deve-se ao fato de $\operatorname{Var}\left[X_{t+1} / X_{t}=x\right]=\operatorname{Var}\left[X_{t-1} / X_{t}=x\right]$.

Calculando $\rho_{\tau}(x)$ dada por (3.5), para $\tau=1$, temos:

$$
\rho_{1}(x)=\frac{-\theta_{1} \sqrt{\gamma_{0}}}{\sqrt{\theta_{1}^{2} \gamma_{0}+\sigma^{2}(x)}}=\frac{-\theta_{1} \sqrt{\sigma_{a}^{2}\left(1+\theta_{1}^{2}\right)}}{\sqrt{\theta_{1}^{2} \sigma_{a}^{2}\left(1+\theta_{1}^{2}\right)+\sigma_{a}^{2}\left(1+\theta_{1}^{4}\right)}}=\frac{-\sqrt{\theta_{1}^{2}\left(1+\theta_{1}^{2}\right)}}{\sqrt{2 \theta_{1}^{4}+\theta_{1}^{2}+1}},
$$

portanto,

$$
\rho_{\tau}(x)= \begin{cases}1 & , \tau=0 \\ -\sqrt{\frac{\theta_{1}^{2}\left(1+\theta_{1}^{2}\right)}{2 \theta_{1}^{4}+\theta_{1}^{2}+1}} & ,|\tau|=1, \quad \forall x \in S . \\ 0 & ,|\tau| \geq 2\end{cases}
$$




\section{Apêndice B - Simulações adicionais da Função de Correlação Local}
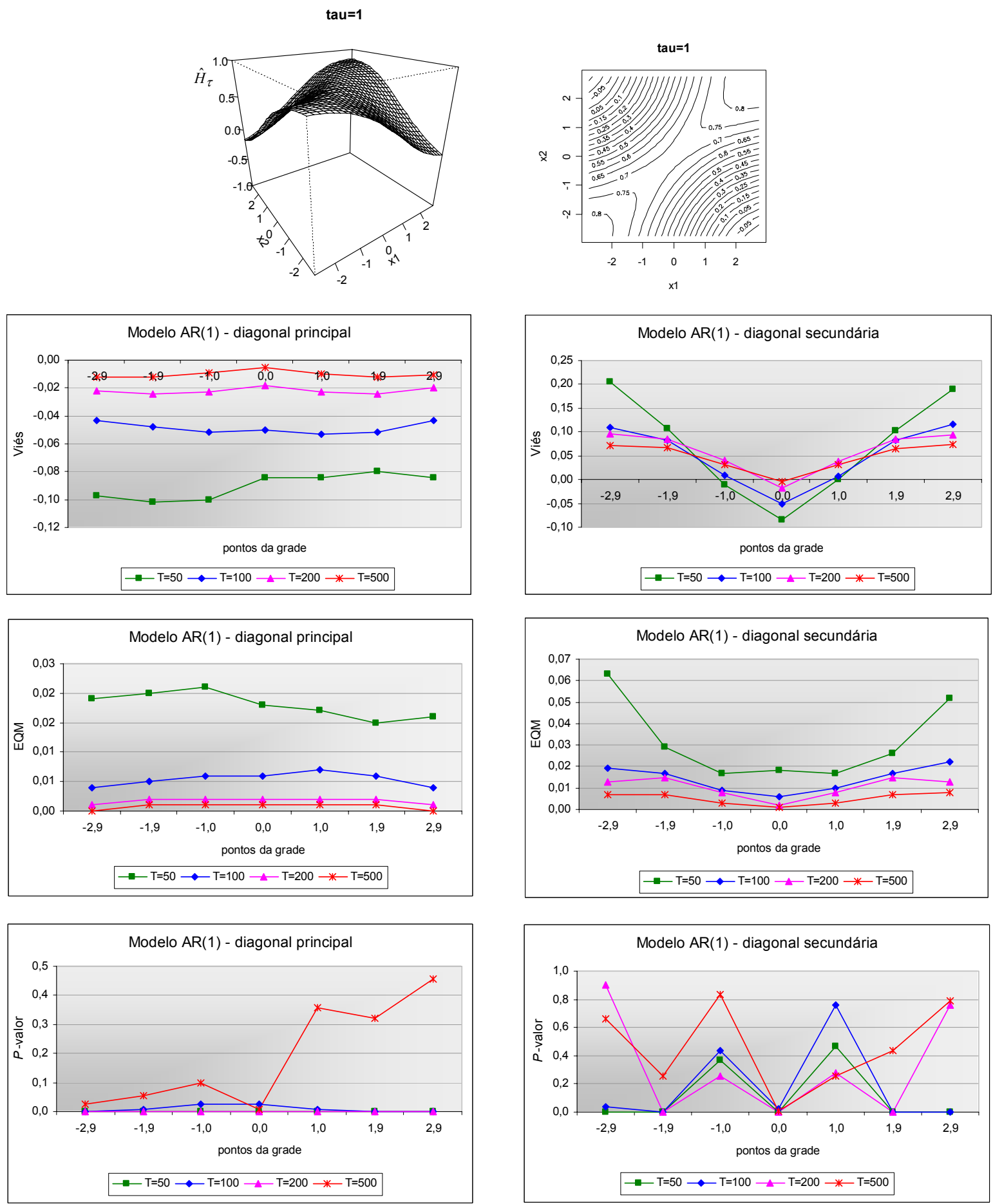

Figura B.1 - Para a f.a.c.1. estimada no lag 1 do modelo simulado AR(1) com 200 repetições de séries, temos: gráfico de perspectiva e curvas de nível $(T=50)$, e gráficos do viés, erro quadrático médio e $p$-valor do teste de normalidade de Jarque Bera, para a diagonal principal e secundária $(T=50, T=100, T=200$ e $T=500)$. 

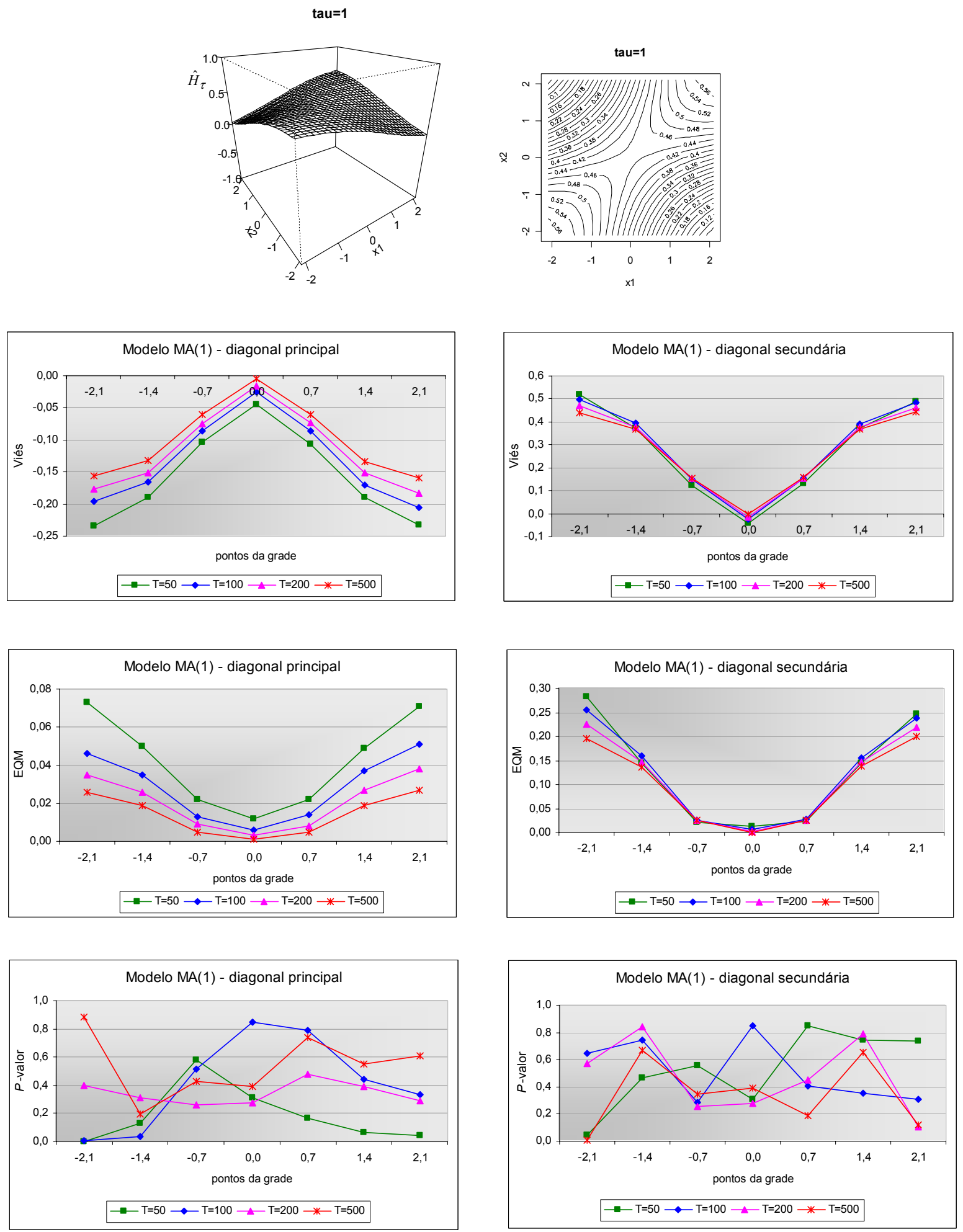

Figura B.2 - Para a f.a.c.l. estimada no lag 1 do modelo simulado MA(1) com 200 repetições de séries, temos: gráfico de perspectiva e curvas de nível $(T=50)$, e gráficos do viés, erro quadrático médio e $p$-valor do teste de normalidade de Jarque Bera, para a diagonal principal e secundária $(T=50, T=100, T=200$ e $T=500)$. 

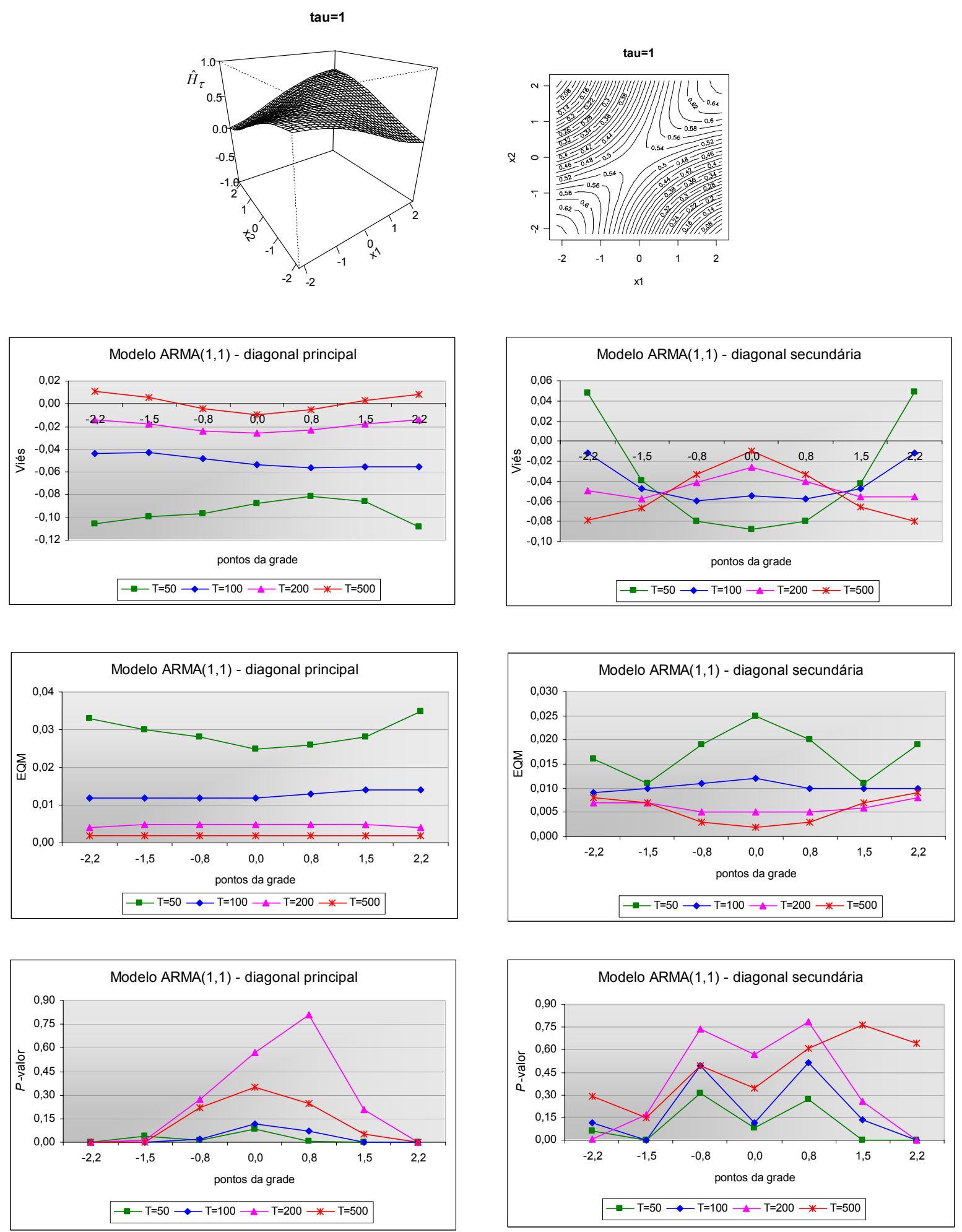

Figura B.3 - Para a f.a.c.l. estimada no lag 1 do modelo simulado ARMA(1,1) com 200 repetições de séries, temos: gráfico de perspectiva e curvas de nível $(T=50)$, e gráficos do viés, erro quadrático médio e $p$-valor do teste de normalidade de Jarque Bera, para a diagonal principal e secundária $(T=50, T=100, T=200$ e $T=500)$. 

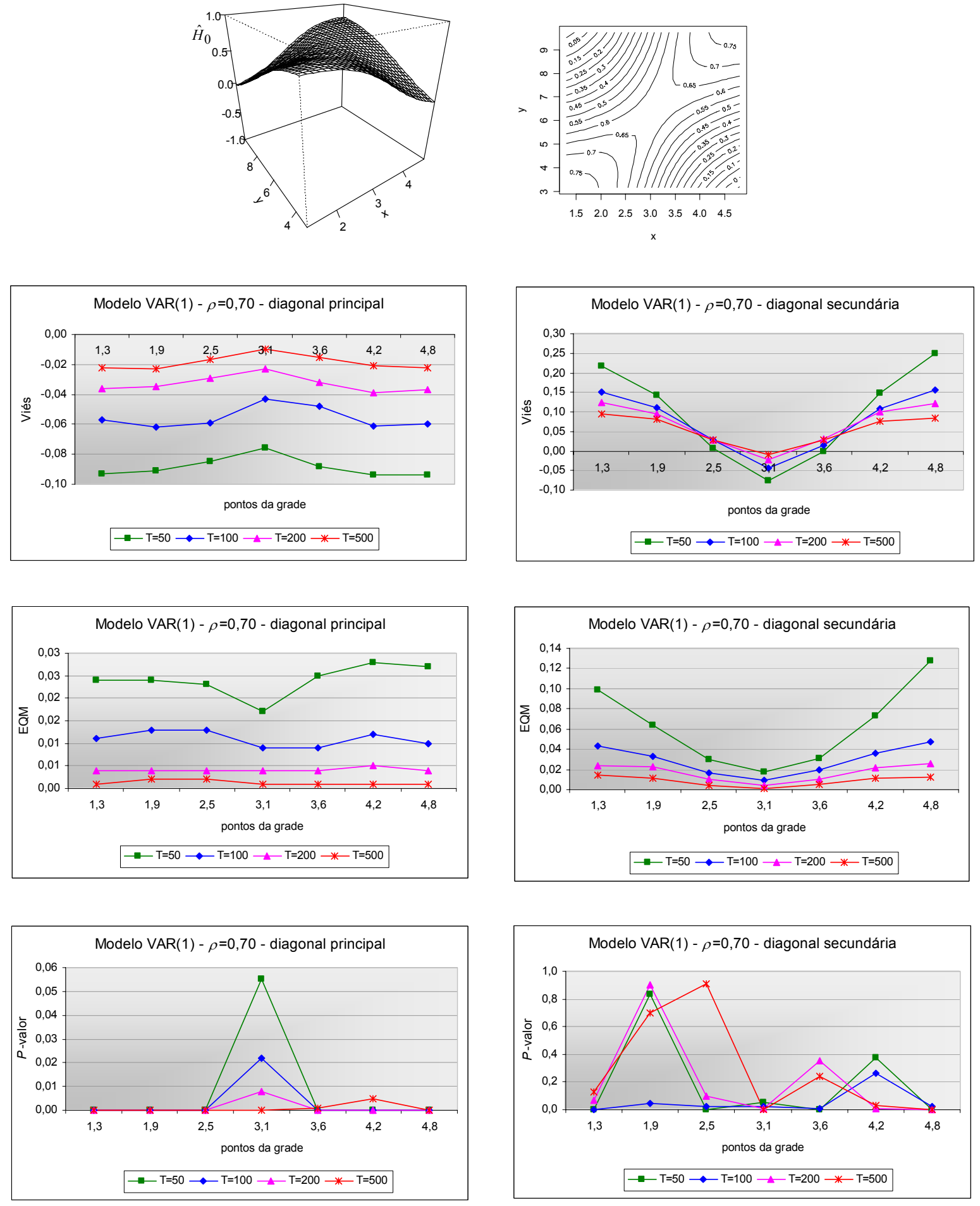

Figura B.4 - Para a f.a.c.l. estimada no lag 1 do modelo simulado VAR(1), $\rho=0,70$, com 200 repetições de séries, temos: gráfico de perspectiva e curvas de nível $(T=50)$, e gráficos do viés, erro quadrático médio e $p$-valor do teste de normalidade de Jarque Bera, para a diagonal principal e secundária $(T=50, T=100, T=200$ e $T=500)$. 

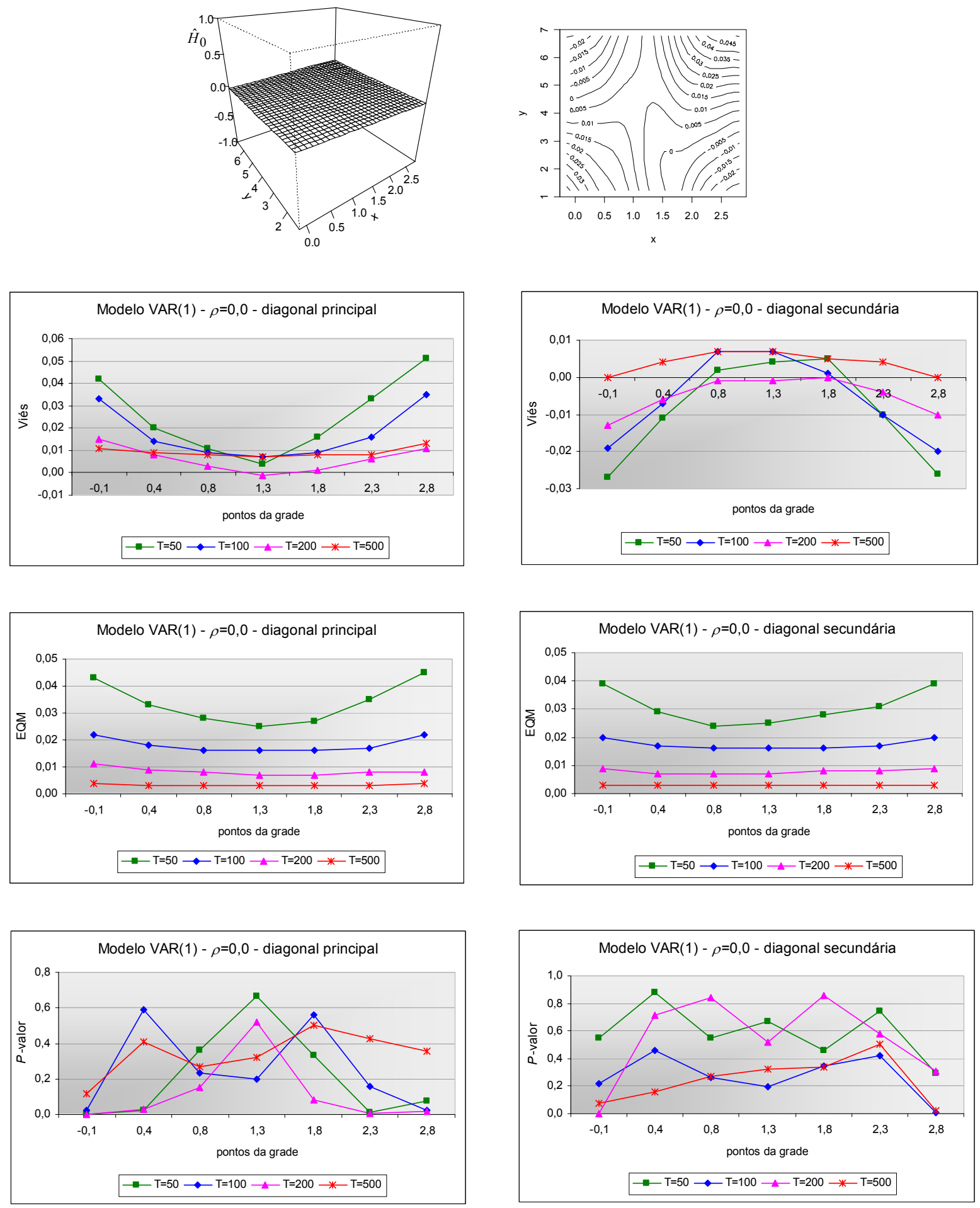

Figura B.5 - Para a f.a.c.l. estimada no lag 1 do modelo simulado VAR(1), $\rho=0,0$, com 200 repetições de séries, temos: gráfico de perspectiva e curvas de nível $(T=50)$, e gráficos do viés, erro quadrático médio e $p$-valor do teste de normalidade de Jarque Bera, para a diagonal principal e secundária $(T=50, T=100, T=200$ e $T=500)$. 

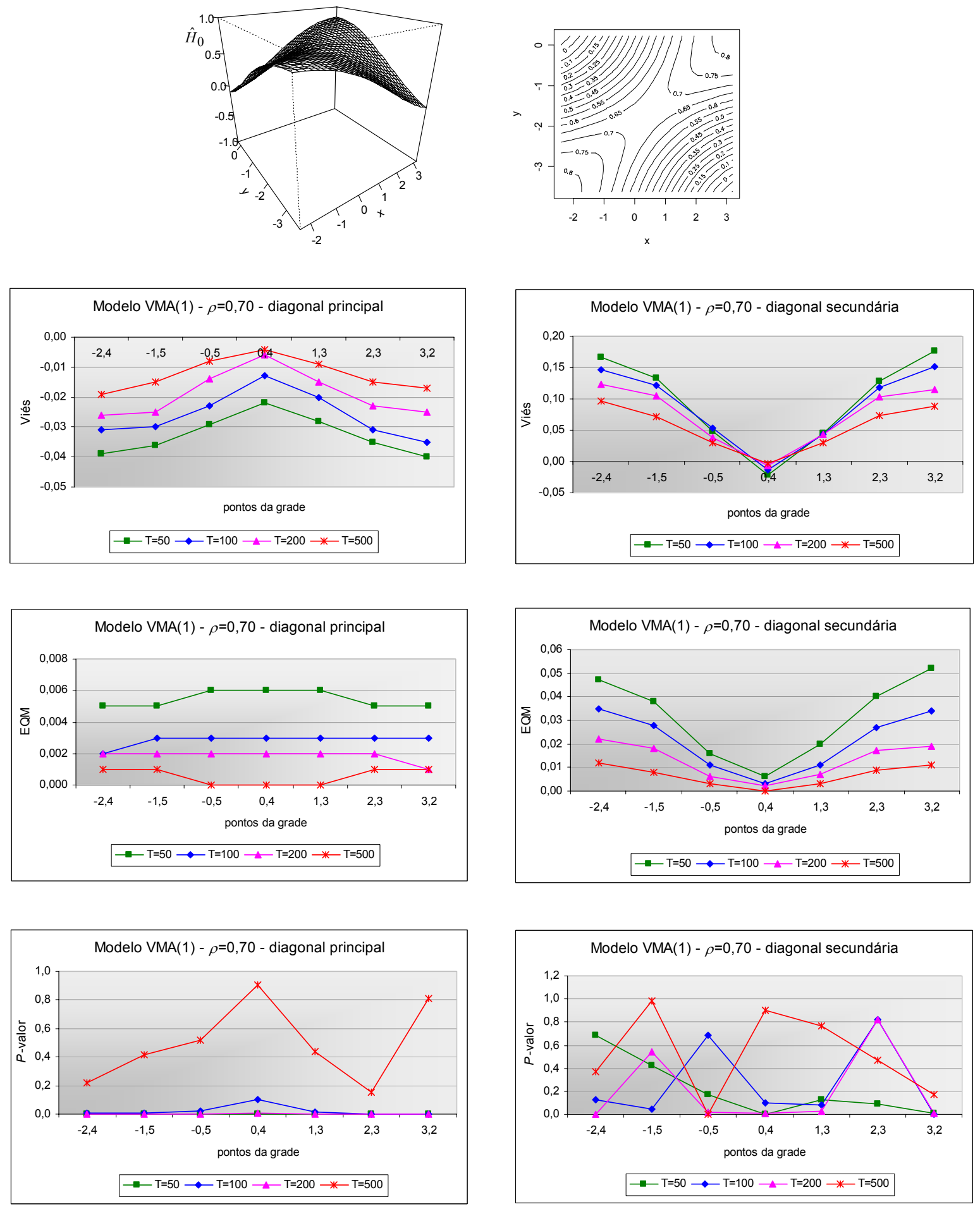

Figura B.6 - Para a f.a.c.l. estimada no lag 1 do modelo simulado $\operatorname{VMA}(1), \rho=0,70$, com 200 repetições de séries, temos: gráfico de perspectiva e curvas de nível $(T=50)$, e gráficos do viés, erro quadrático médio e $p$-valor do teste de normalidade de Jarque Bera, para a diagonal principal e secundária $(T=50, T=100, T=200$ e $T=500)$. 

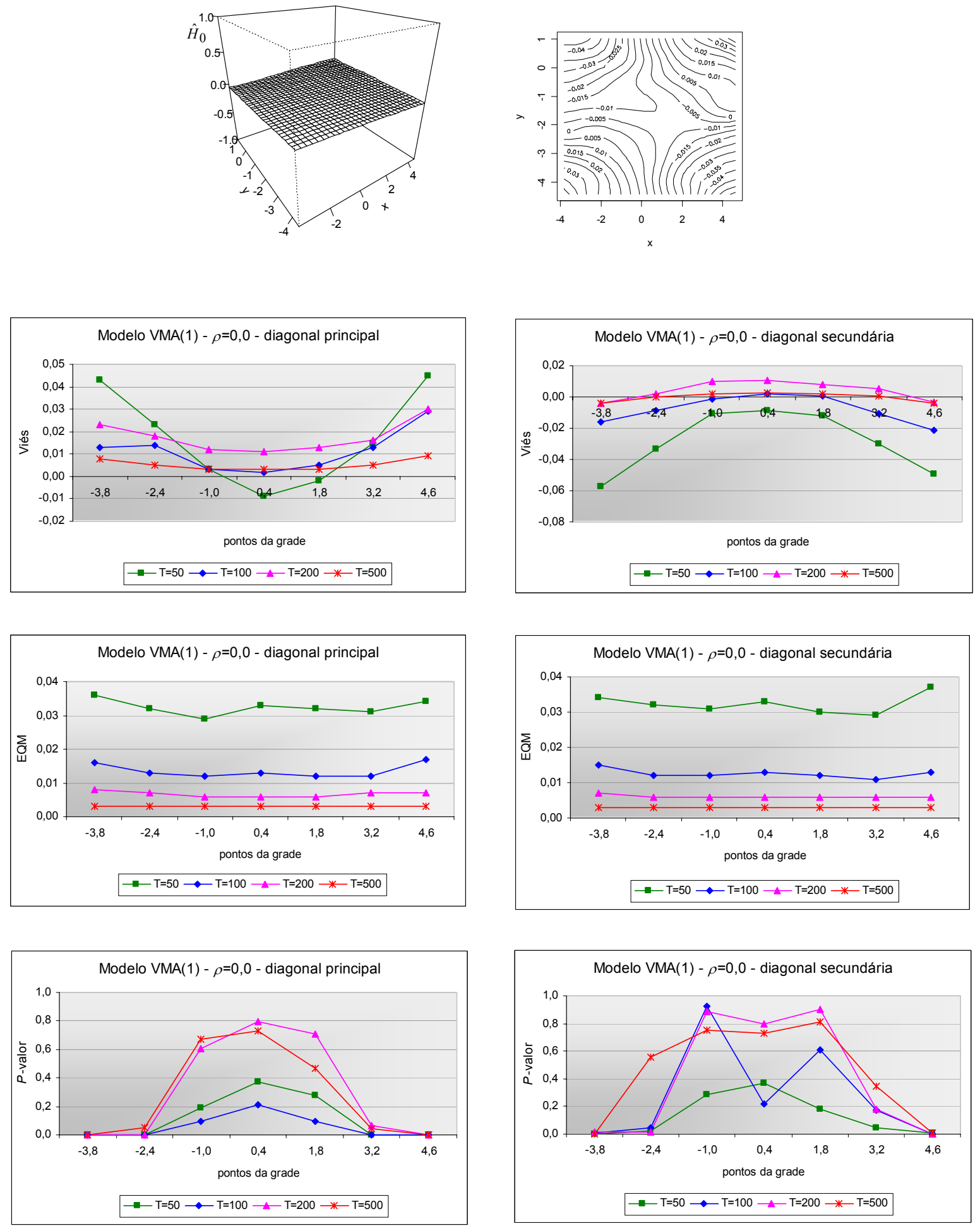

Figura B.7 - Para a f.a.c.l. estimada no lag 1 do modelo simulado $\operatorname{VMA}(1), \rho=0,0$, com 200 repetições de séries, temos: gráfico de perspectiva e curvas de nível $(T=50)$, e gráficos do viés, erro quadrático médio e $p$-valor do teste de normalidade de Jarque Bera, para a diagonal principal e secundária $(T=50, T=100, T=200$ e $T=500)$. 


\section{Apêndice C - Simulações adicionais da Curva de Correlação}
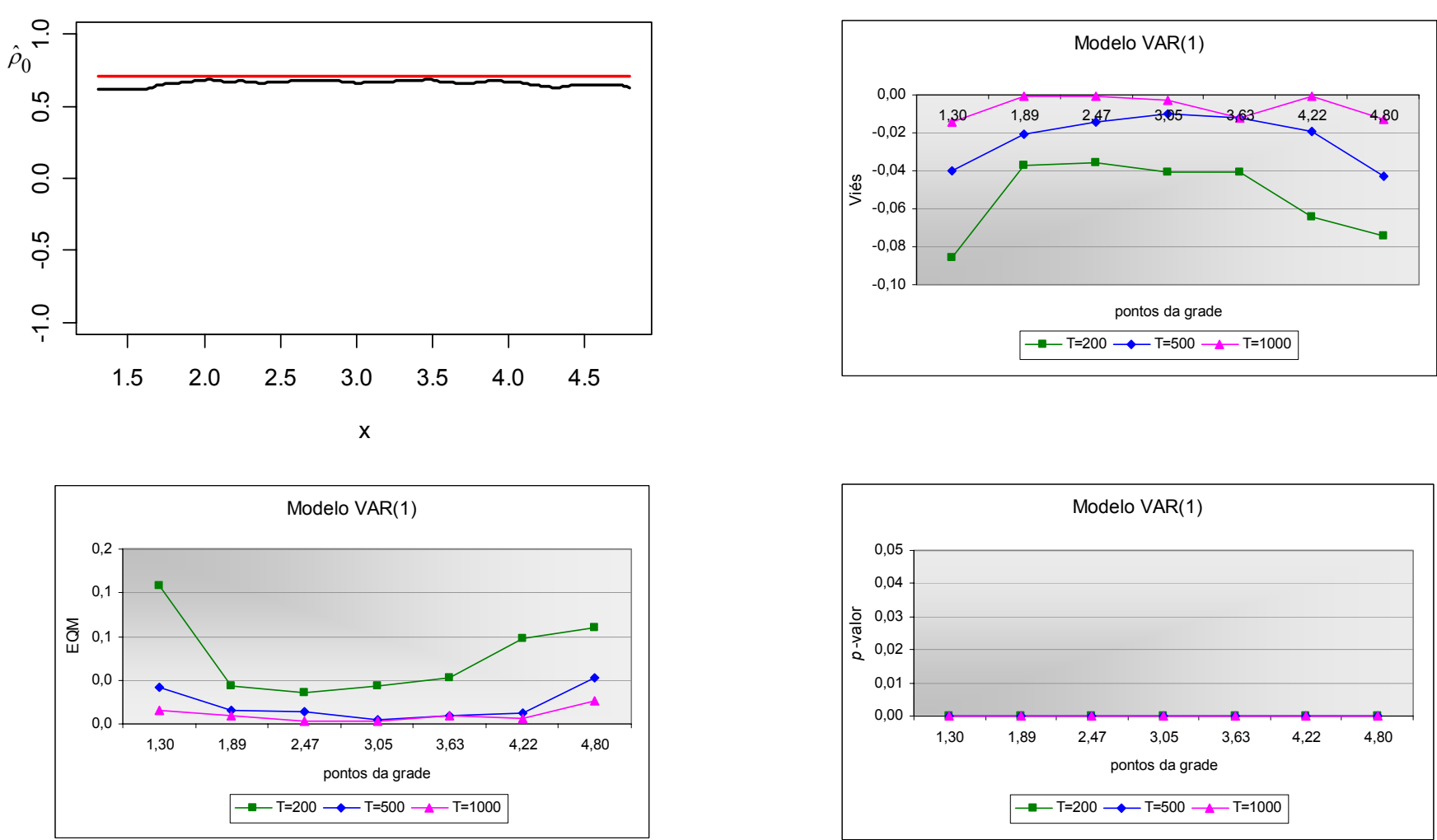

Figura C.1 - Para curva de correlação cruzada contemporânea do modelo simulado VAR(1) obtida através de 200 repetições de séries, temos: curva estimada (linha) e teórica (reta) para $T=200$, e gráficos do viés, erro quadrático médio e

$p$-valor do teste de normalidade de Jarque Bera para $T=200, T=500$ e $T=1.000$. 

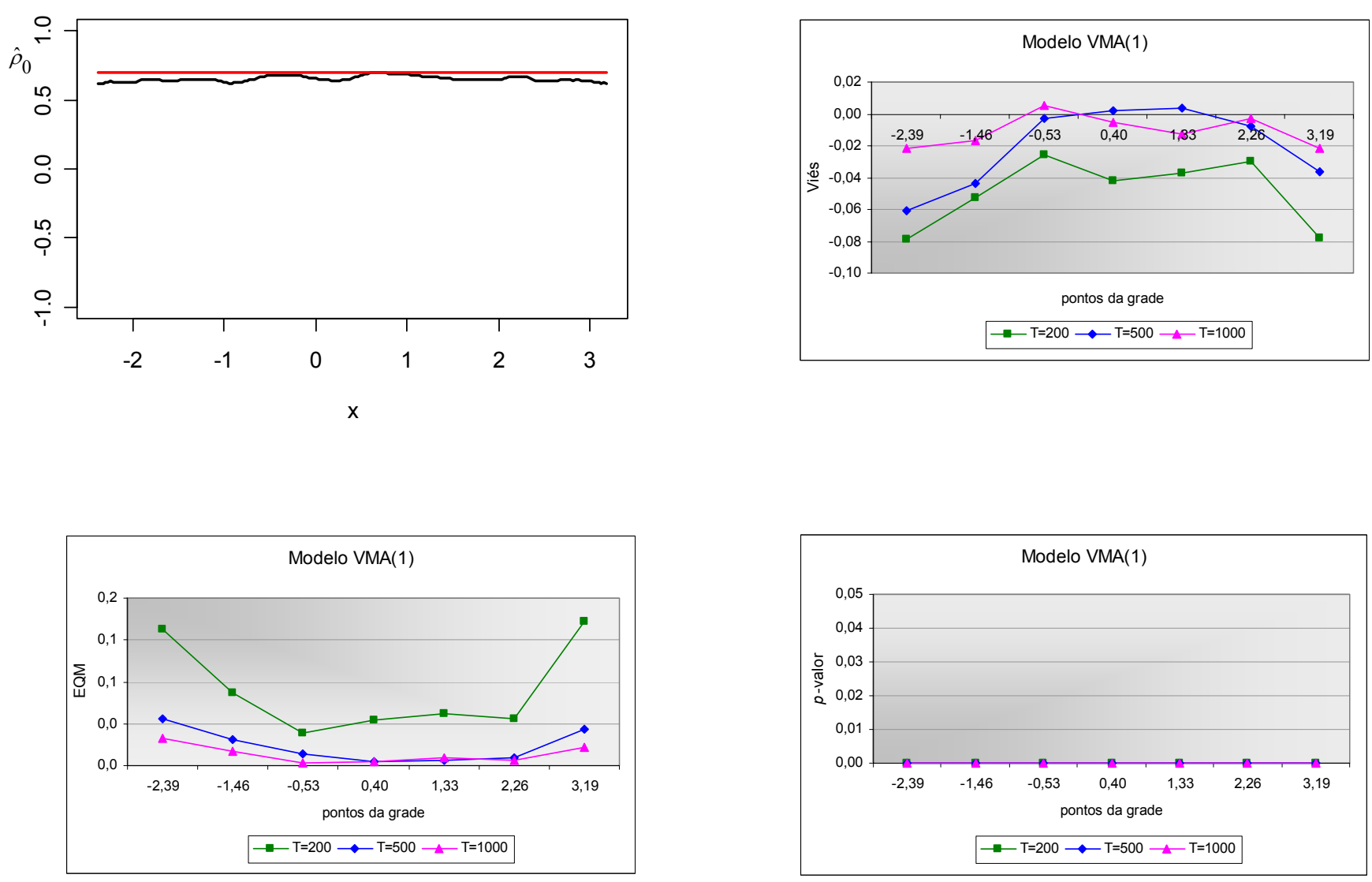

Figura C.2 - Para a curva de correlação cruzada contemporânea do modelo simulado VMA(1) com 200 repetições de séries, temos: curva estimada (linha) e teórica (reta) para $T=200$, e gráficos do viés, erro quadrático médio e $p$-valor do teste de normalidade de Jarque Bera para $T=200, T=500$ e $T=1.000$. 

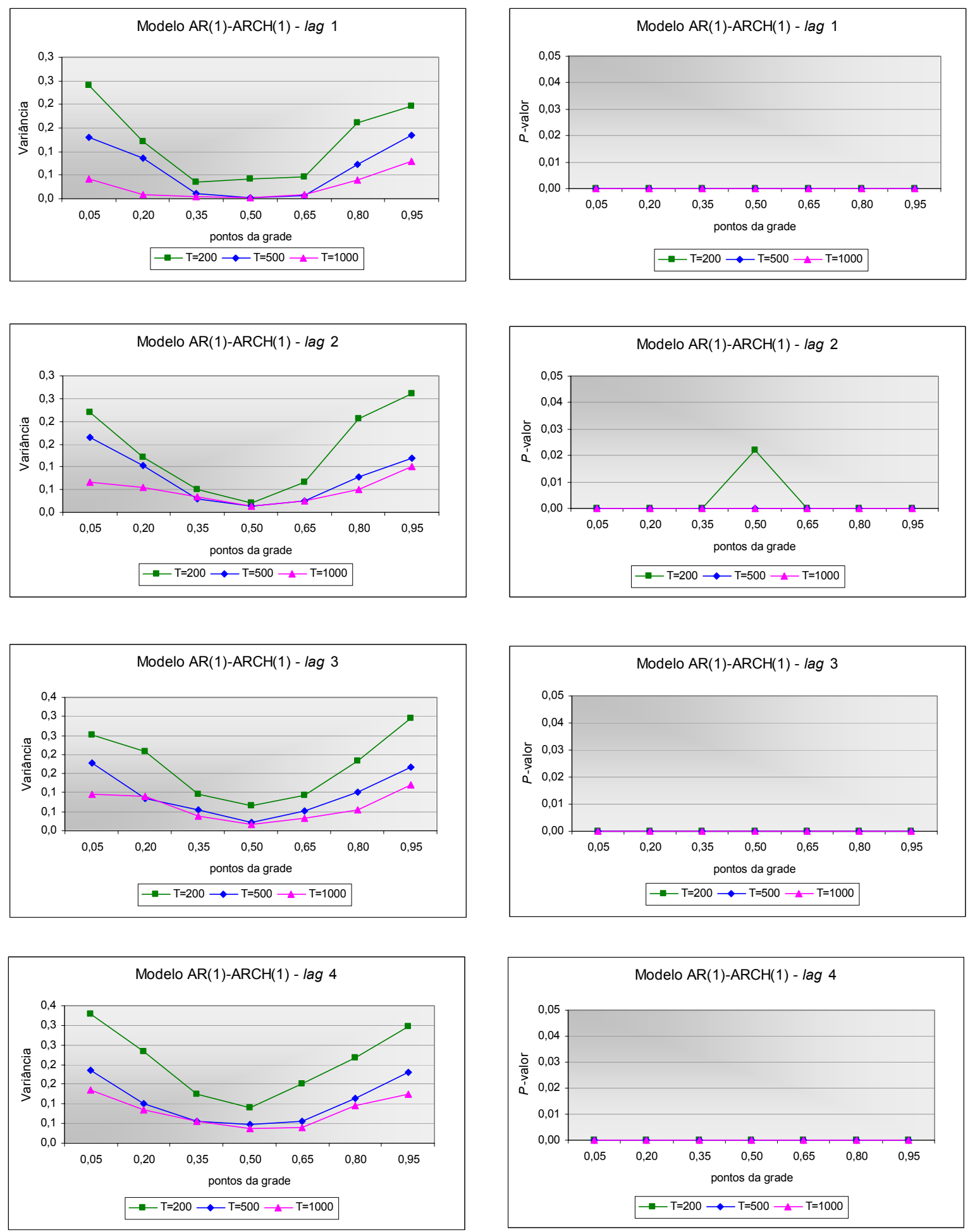

Figura C.3 - Para a curva de autocorrelação estimada no $\operatorname{lag}$ 1, 2, 3 e 4, utilizando 200 séries observadas de um modelo $\mathrm{AR}(1)-\mathrm{ARCH}(1)$, temos: os gráficos da variância da curva e $p$-valor do teste de normalidade de Jarque Bera para $T=200, T=500$ e $T=1.000$. 


\section{Apêndice D - Simulações adicionais da Função de Sibuya}
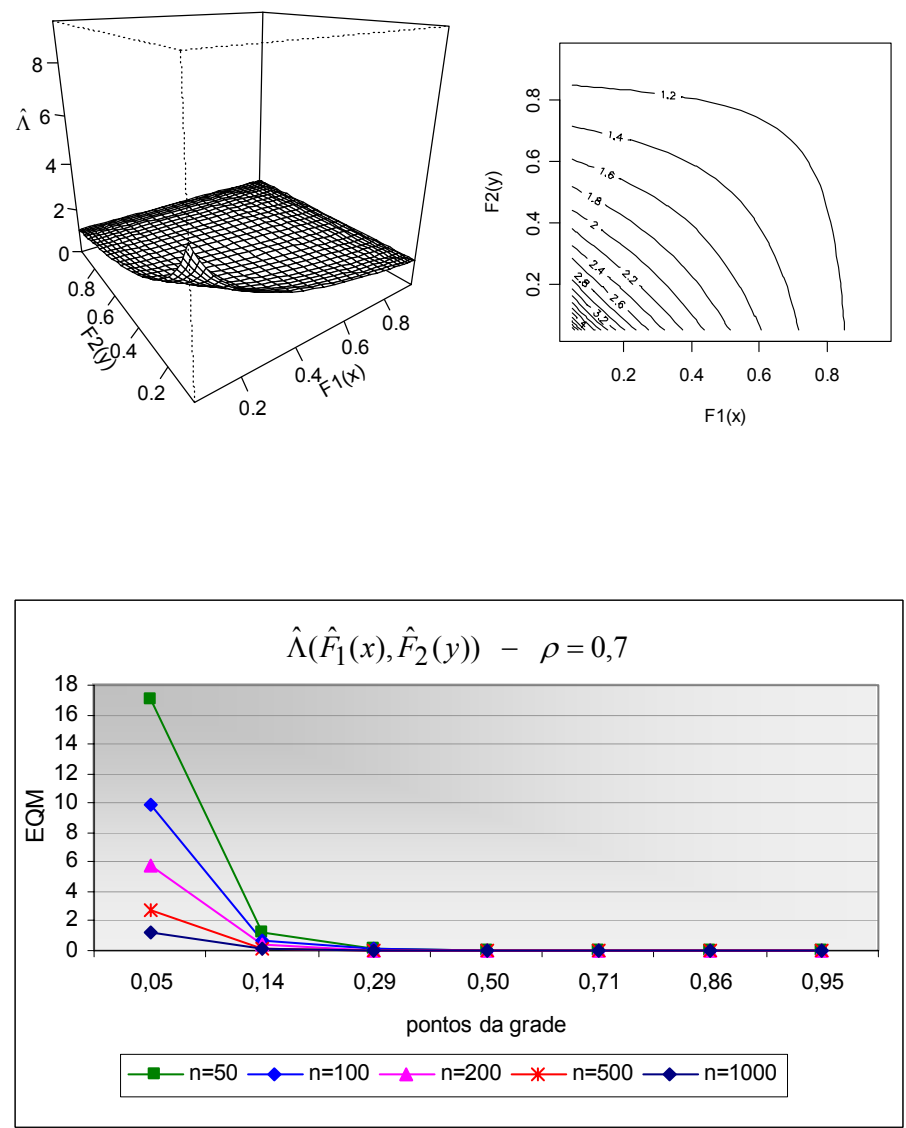
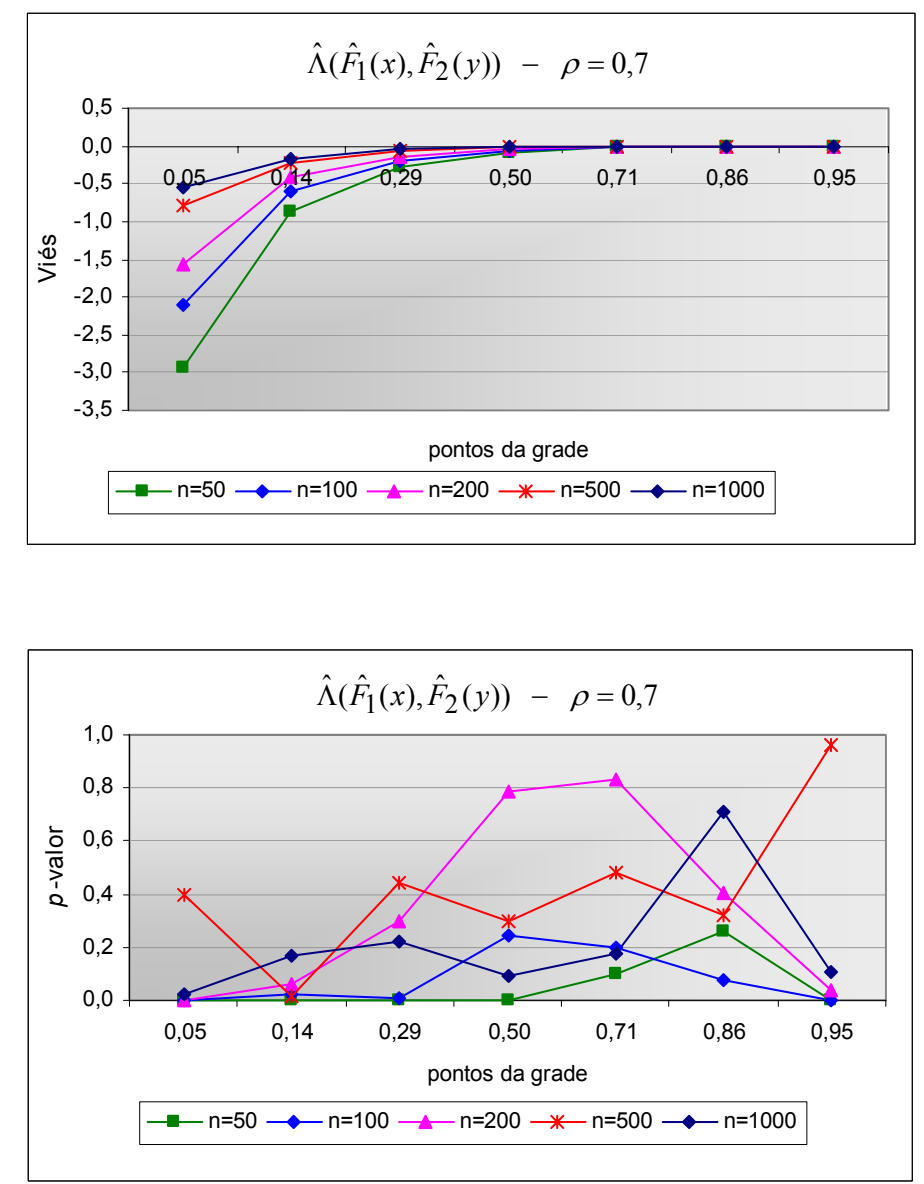

Figura D.1 - Função de dependência de Sibuya via funções de distribuição: gráfico de perspectiva e de curvas de nível estimadas para amostras de tamanho 50 , além do comportamento do viés, erro quadrático médio e $p$-valor do teste de normalidade de Jarque Bera para diferentes tamanhos de amostras observadas de um vetor aleatório $(X, Y)$ com distribuição normal de média $(3,05 ; 6,44)^{\prime}$ e vec $(\Sigma)=(1,13 ; 1,49 ; 1,49 ; 3,99)^{\prime}$ (correlação 0,70$)$. 

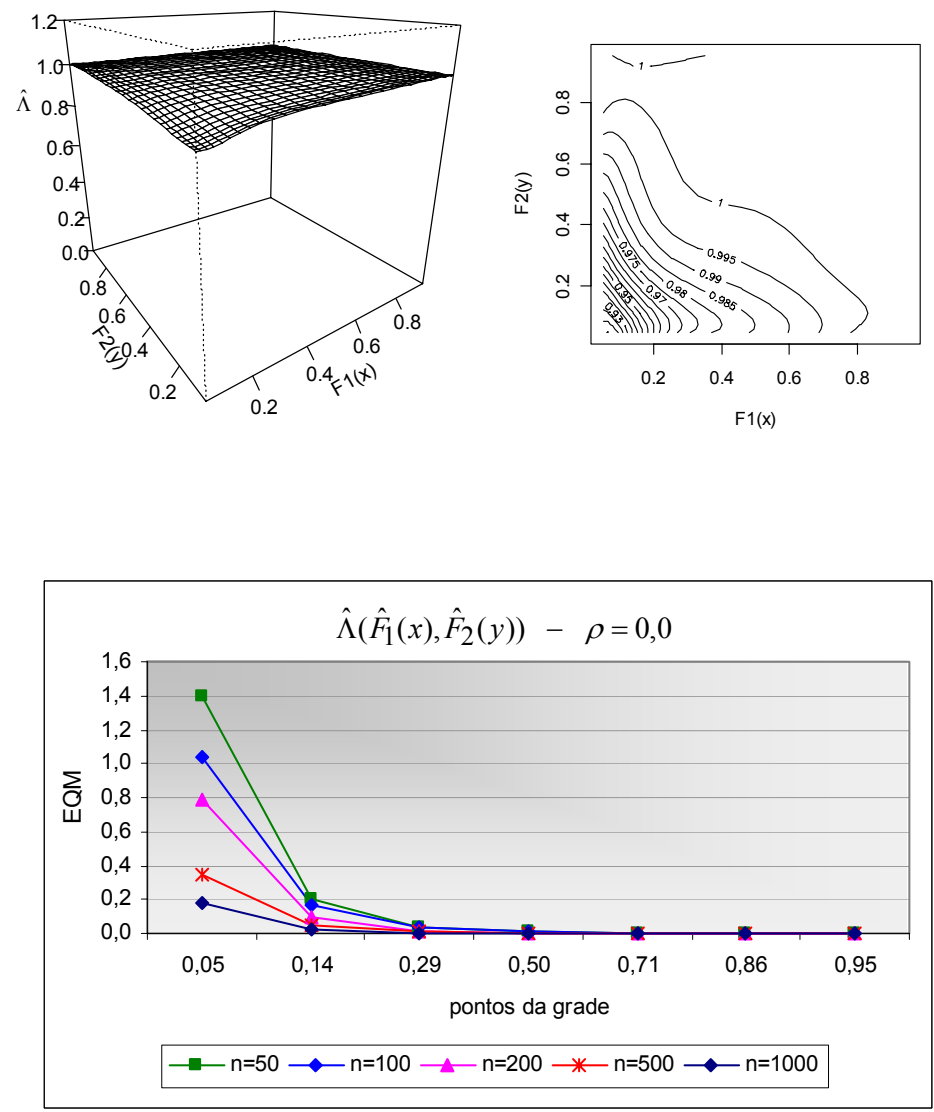
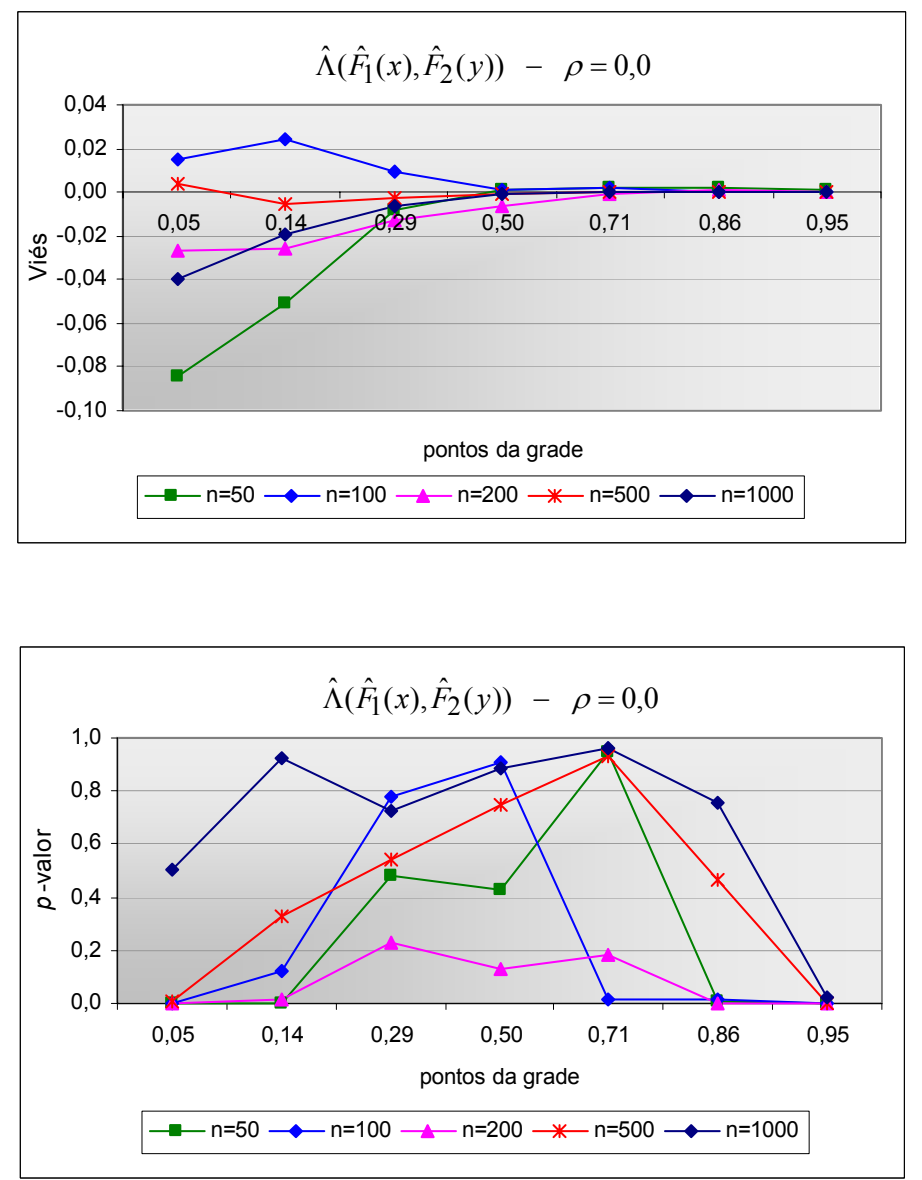

Figura D.2 - Função de dependência de Sibuya via funções de distribuição: gráfico de perspectiva e de curvas de nível estimadas para amostras de tamanho

50, além do comportamento do viés, erro quadrático médio e $p$-valor do teste de normalidade de Jarque Bera para diferentes tamanhos de amostras observadas de um vetor aleatório $(X, Y)$ com distribuição normal de média $(1,33 ; 4)^{\prime}$ e vec $(\Sigma)=(0,80 ; 0 ; 0 ; 2,86)^{\prime}($ correlação 0,0$)$. 

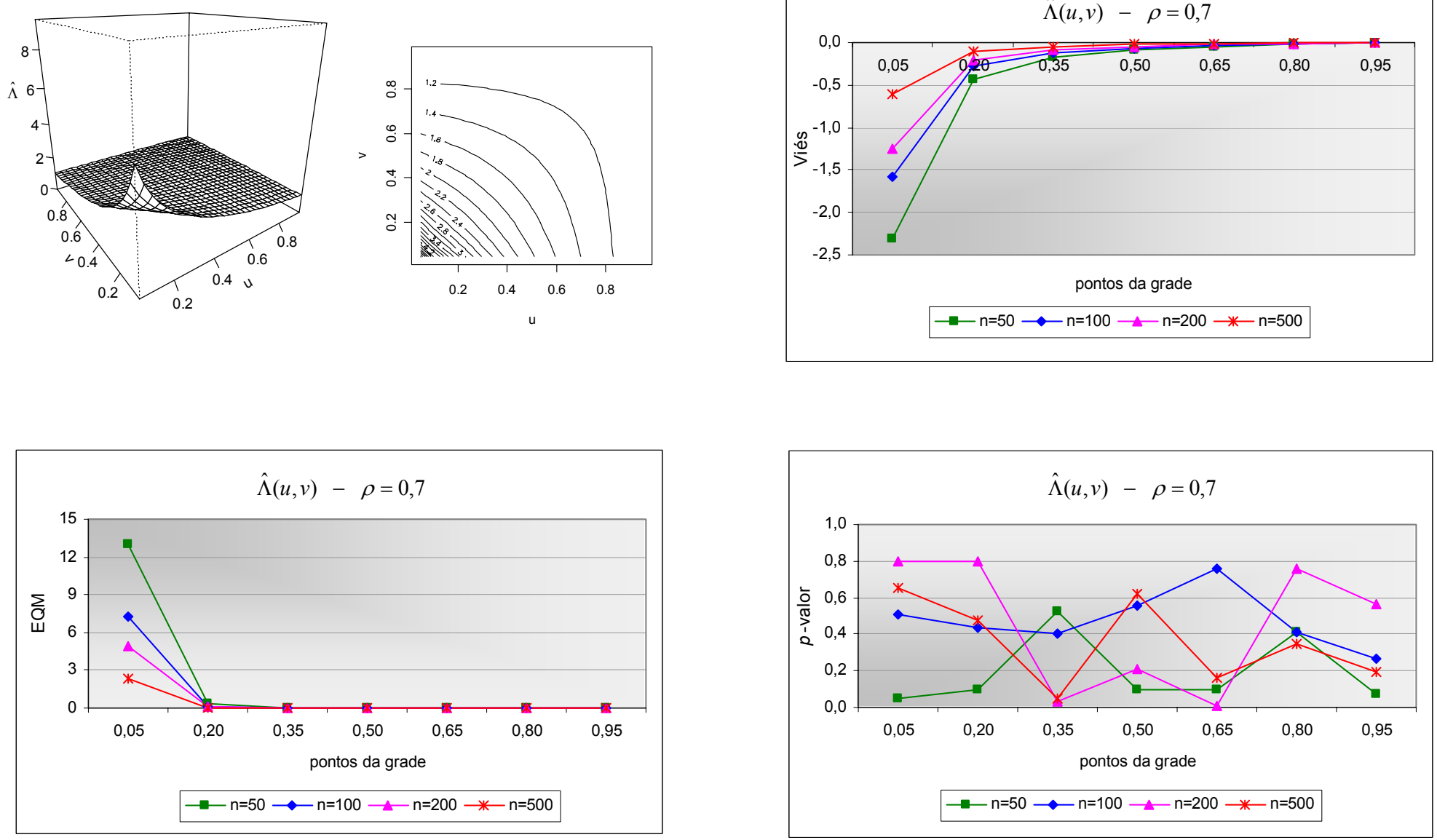

Figura D.3 - Função de dependência de Sibuya via cópula: gráfico de perspectiva e de curvas de nível estimadas para amostras de tamanho 50, além do comportamento do viés, erro quadrático médio e $p$-valor do teste de normalidade de Jarque Bera para diferentes tamanhos de amostras observadas de um vetor aleatório $(X, Y)$ com distribuição normal de média $(3,05 ; 6,44)^{\prime}$ e vec $(\Sigma)=(1,13 ; 1,49 ; 1,49 ; 3,99)^{\prime}($ correlação 0,70$)$. 

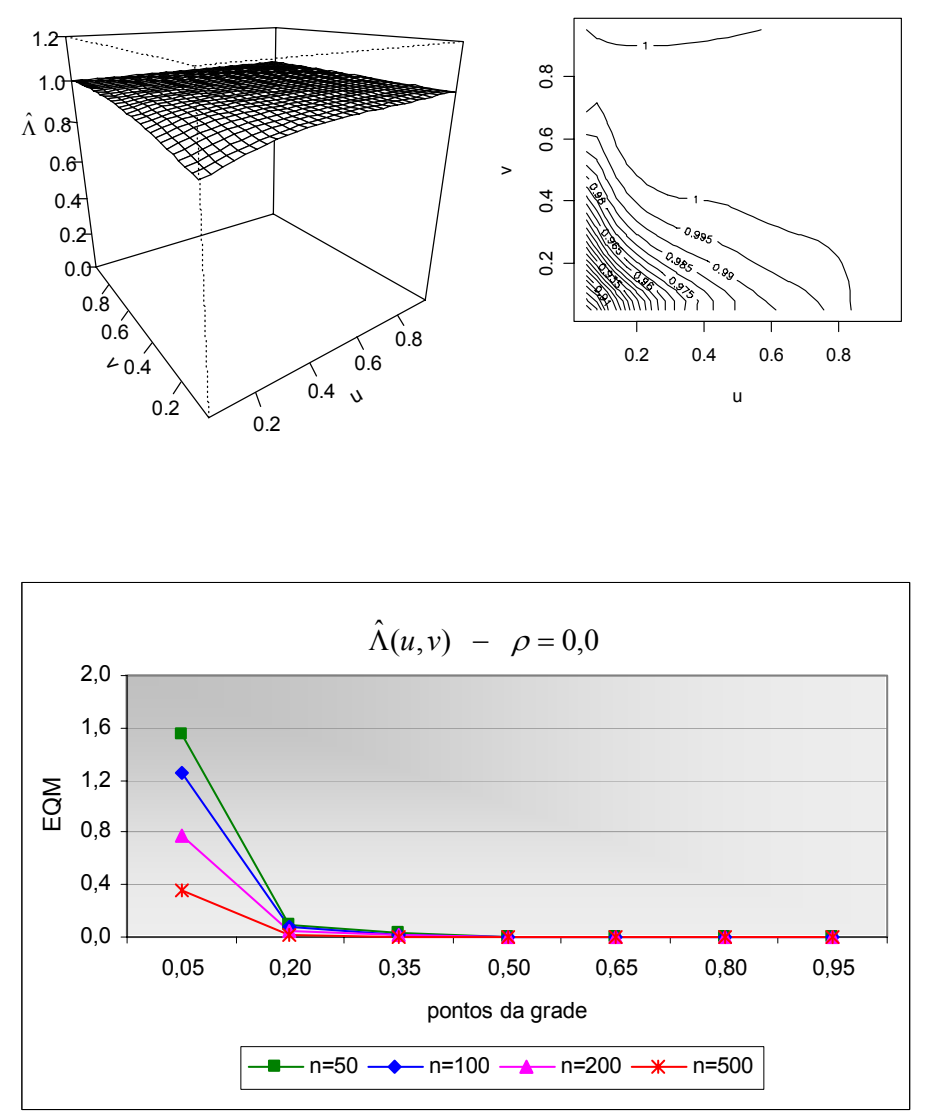
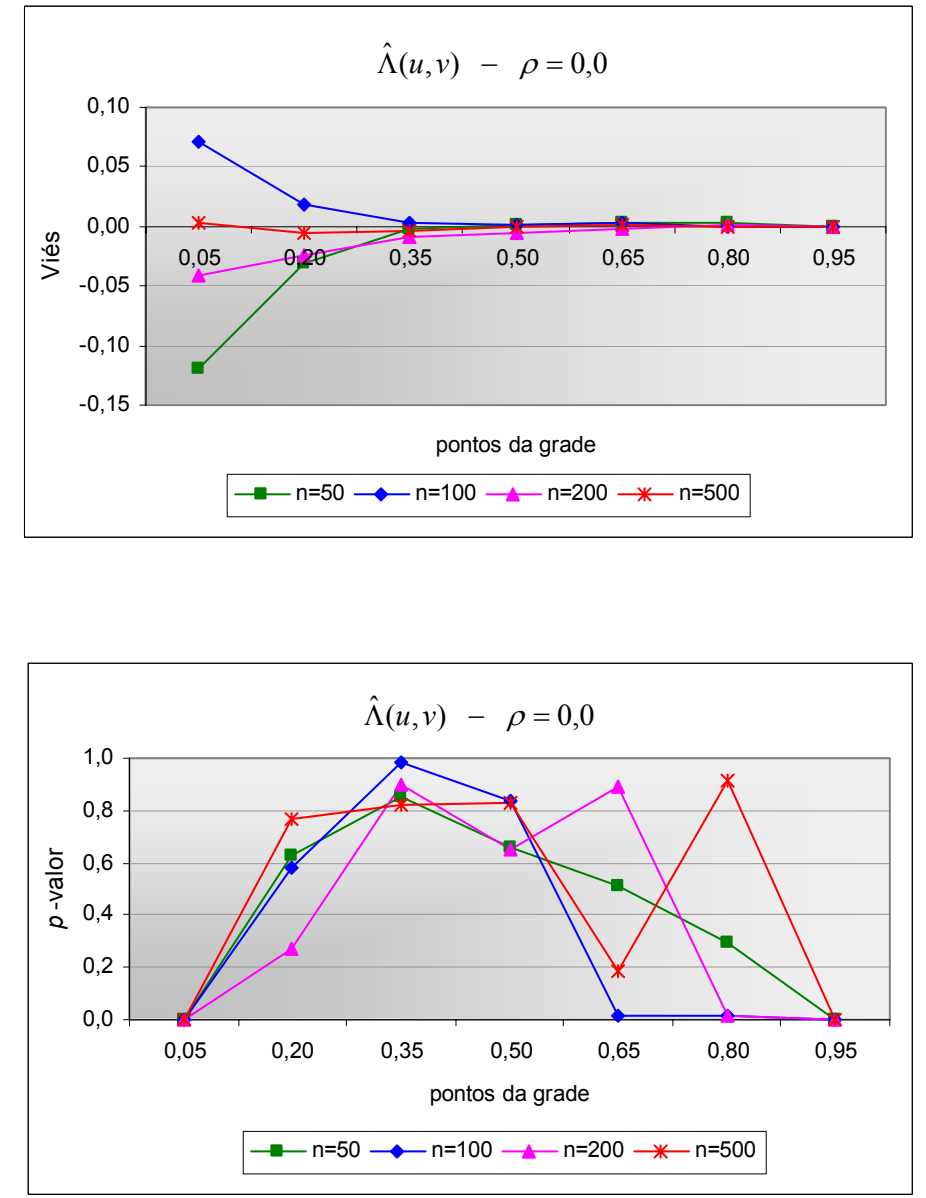

Figura D.4 - Função de dependência de Sibuya via cópula: gráfico de perspectiva e de curvas de nível estimadas para amostras de tamanho 50, além do comportamento do viés, erro quadrático médio e $p$-valor do teste de normalidade de Jarque Bera para diferentes tamanhos de amostras observadas de um vetor aleatório $(X, Y)$ com distribuição normal de média $(1,33 ; 4)^{\prime}$ e vec $(\Sigma)=(0,80 ; 0 ; 0 ; 2,86)^{\prime}($ correlação 0,0$)$. 

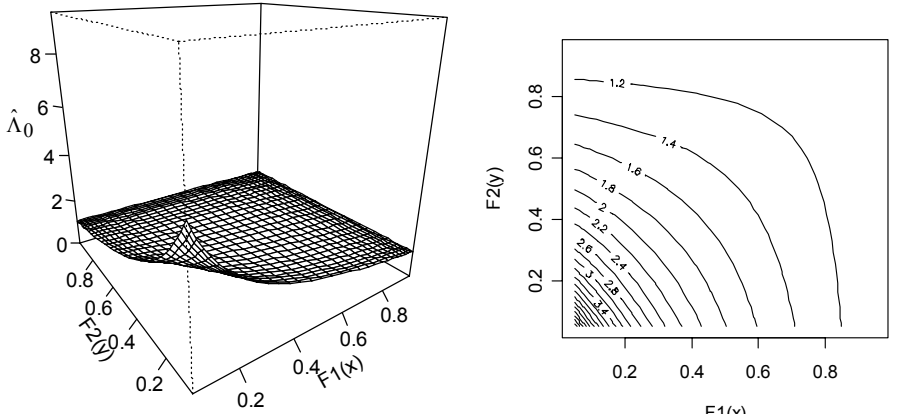

$\mathrm{F} 1(\mathrm{x})$

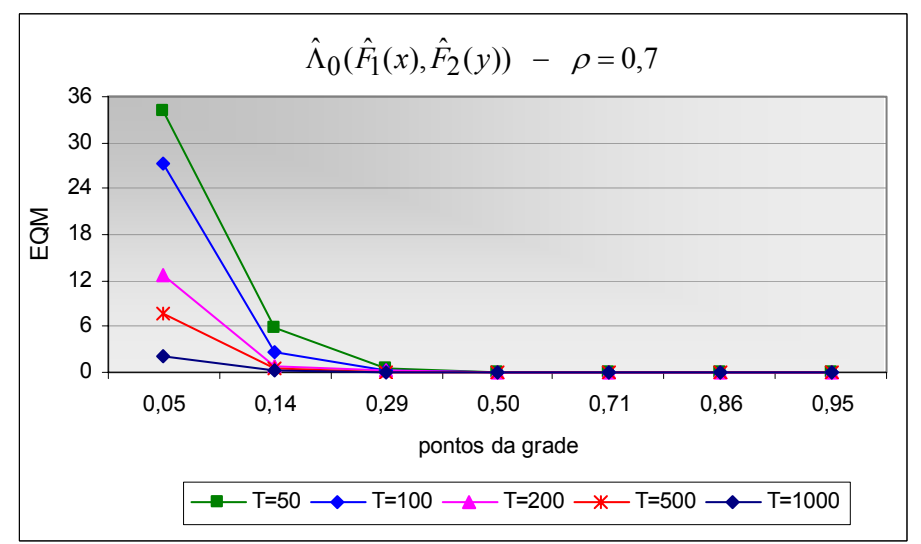

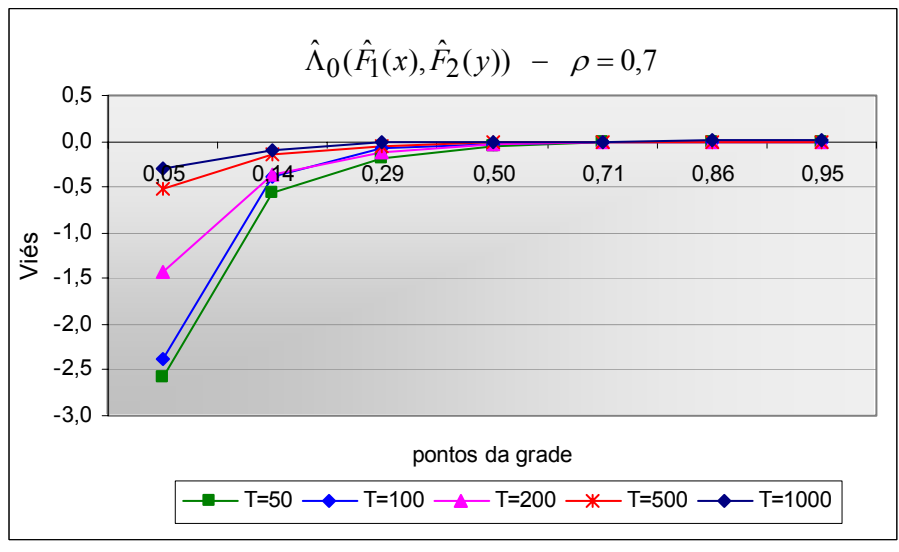

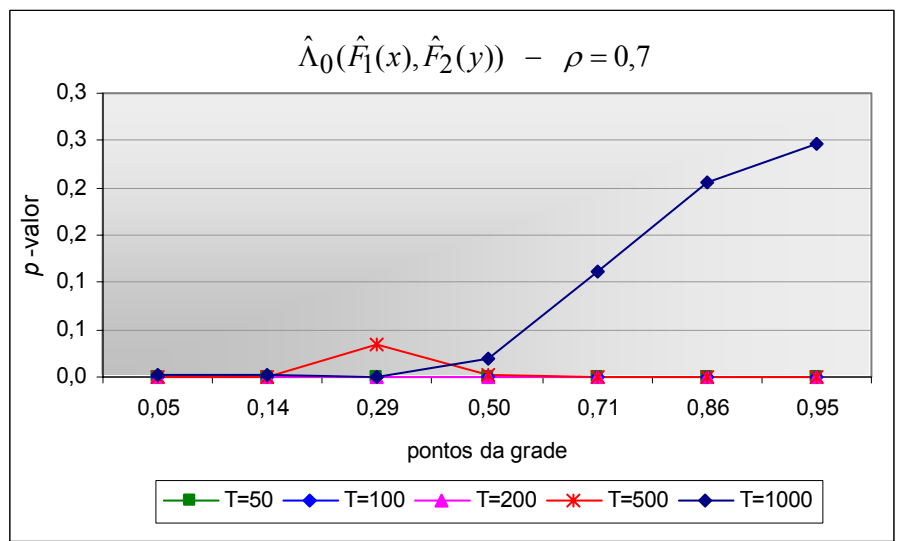

Figura D.5 - Função de dependência cruzada contemporânea local via funções de distribuição: gráfico de perspectiva e de curvas de nível estimadas para amostras de tamanho 50, além do comportamento do viés, erro quadrático médio e $p$-valor do teste de normalidade de Jarque Bera para diferentes tamanhos de amostras observadas de um modelo VAR(1) com média $(3,05 ; 6,44)^{\prime}$ e vec $(\Gamma(0))=(1,13 ; 1,49 ; 1,49 ; 3,99)^{\prime}$ (correlação 0,70$)$. 

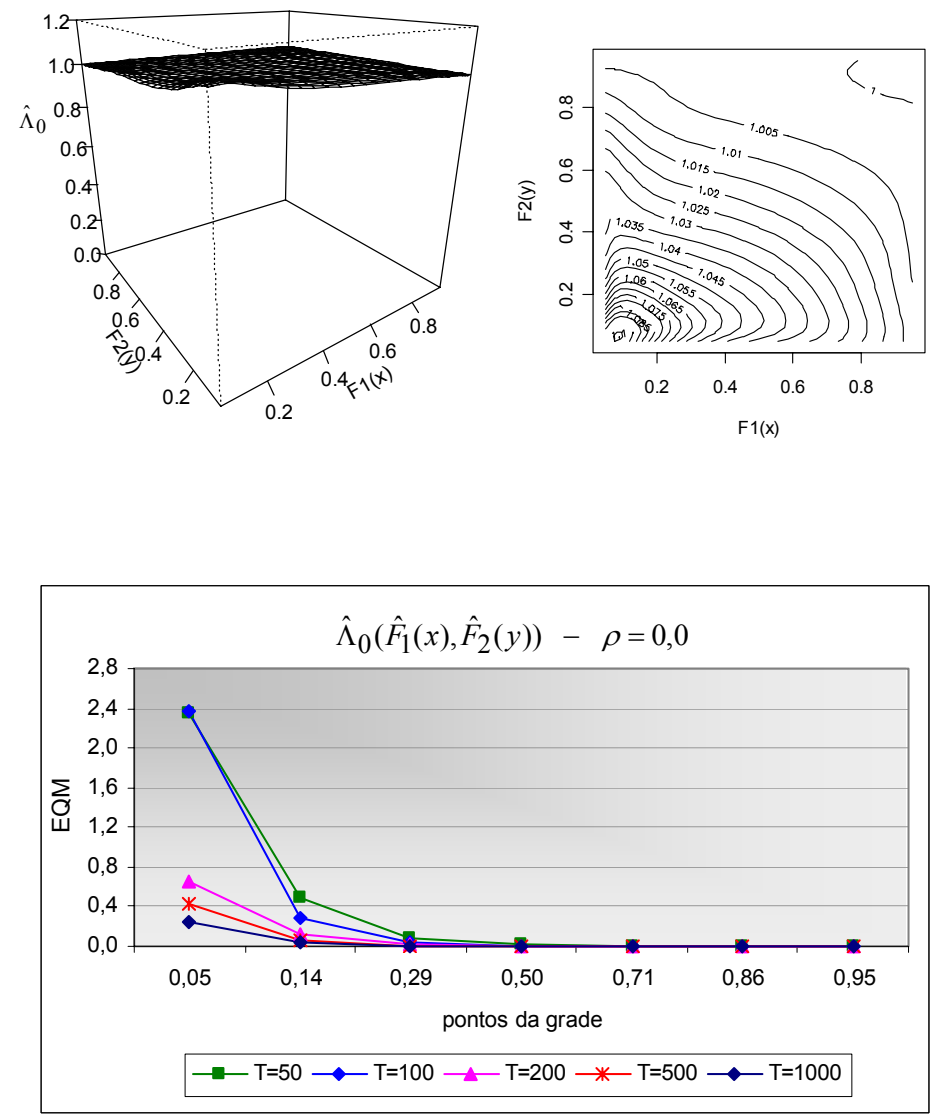
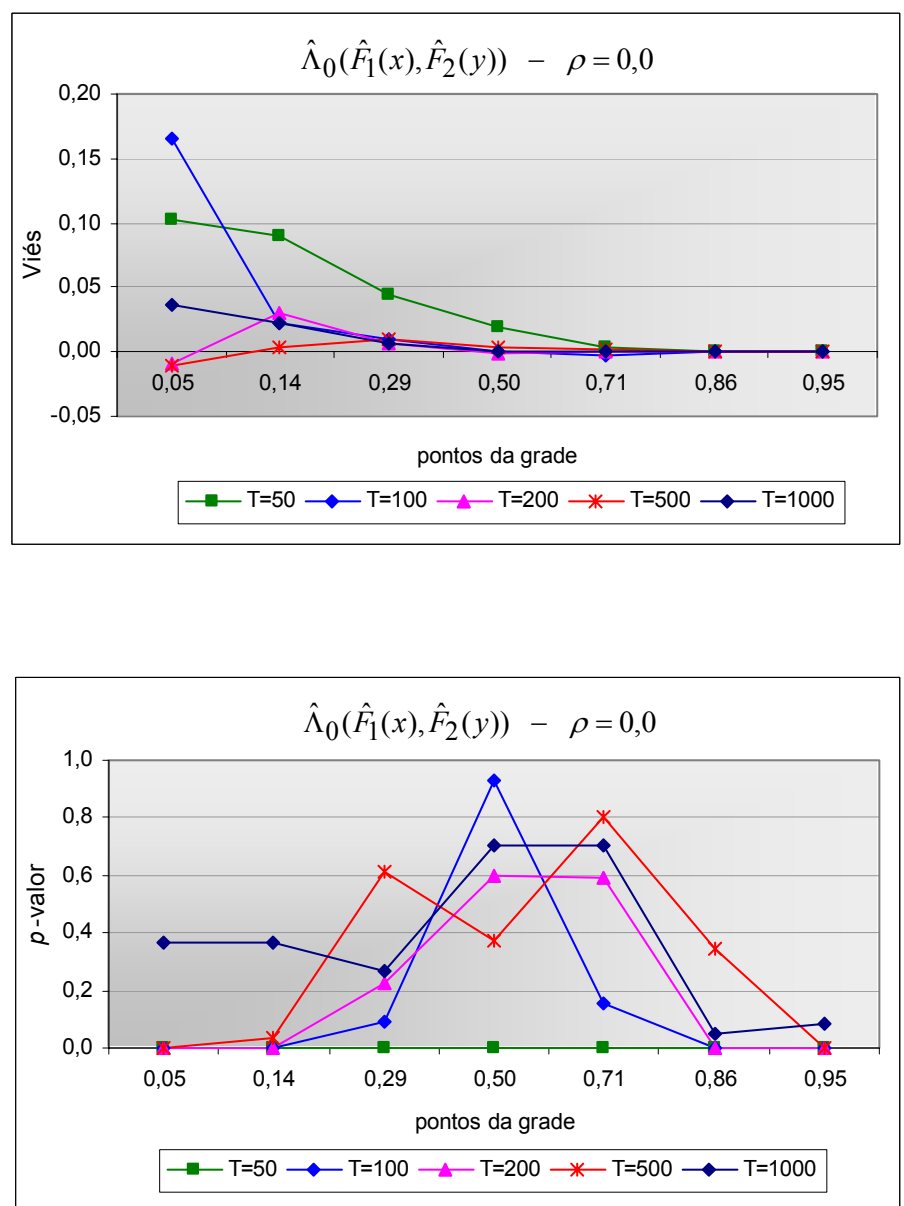

Figura D.6 - Função de dependência cruzada contemporânea local via funções de distribuição: gráfico de perspectiva e de curvas de nível estimadas para amostras de tamanho 50, além do comportamento do viés, erro quadrático médio e $p$-valor do teste de normalidade de Jarque Bera para diferentes tamanhos de amostras observadas de um modelo VAR(1) com média $(1,33 ; 4)^{\prime}$ e vec $(\Sigma)=(0,80 ; 0 ; 0 ; 2,86)^{\prime}($ correlação 0,0$)$. 

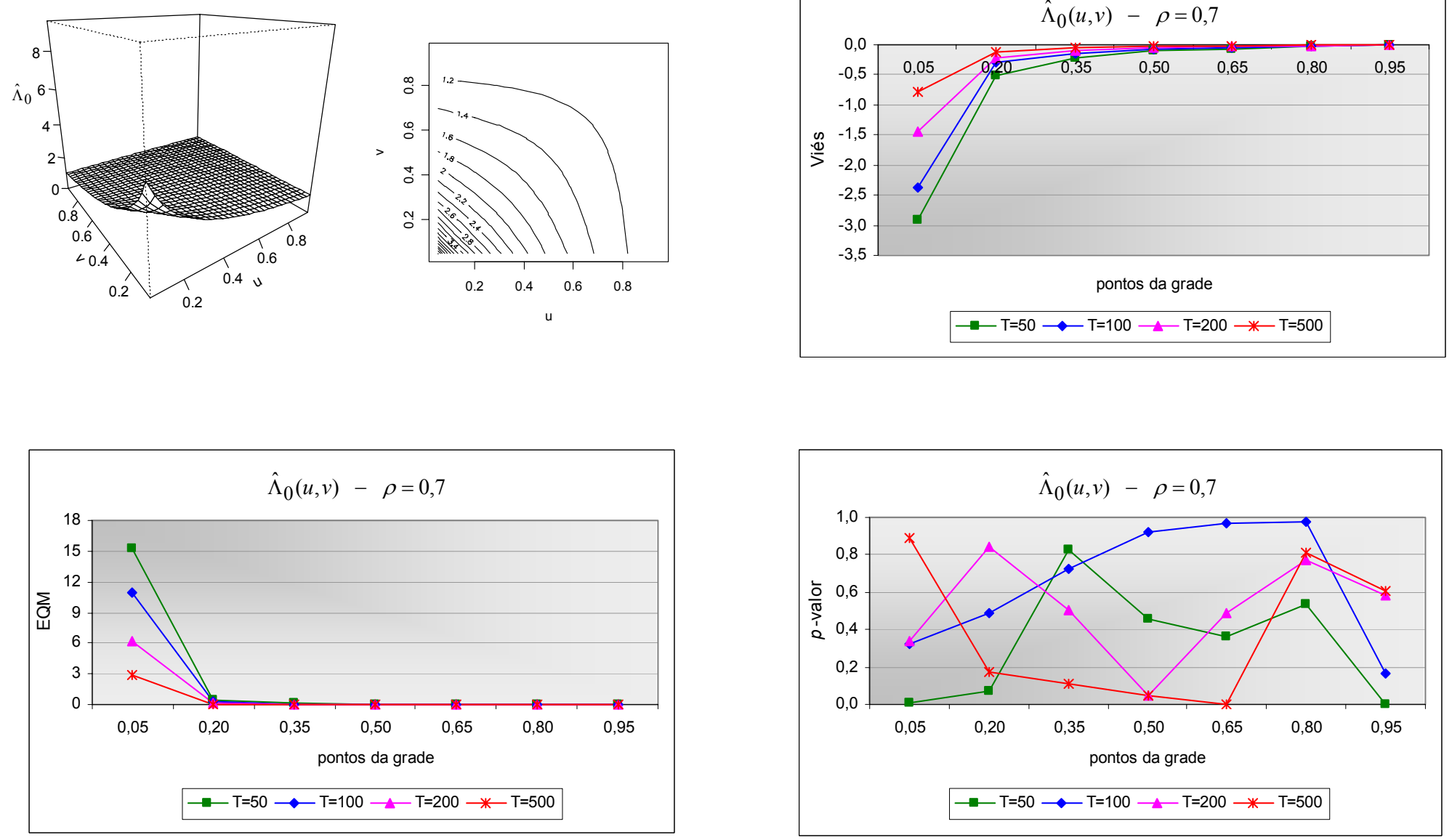

Figura D.7 - Função de dependência cruzada contemporânea local via cópula: gráfico de perspectiva e de curvas de nível estimadas para amostras de tamanho 50, além do comportamento do viés, erro quadrático médio e $p$-valor do teste de normalidade de Jarque Bera para diferentes tamanhos de amostras observadas de um modelo VAR(1) com média $(3,05 ; 6,44)^{\prime}$ e vec( $\left.\Gamma(0)\right)=(1,13 ; 1,49 ; 1,49 ; 3,99)^{\prime}$ (correlação 0,70$)$. 

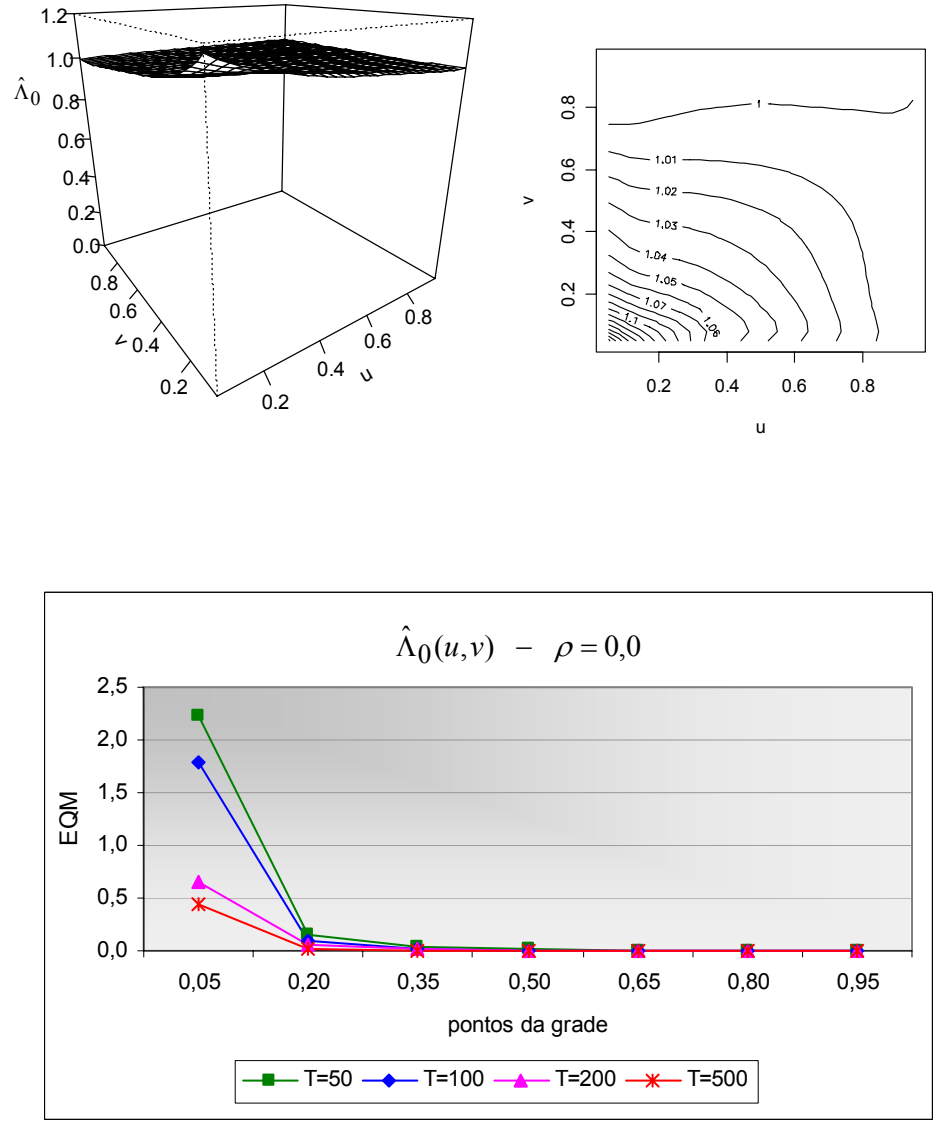
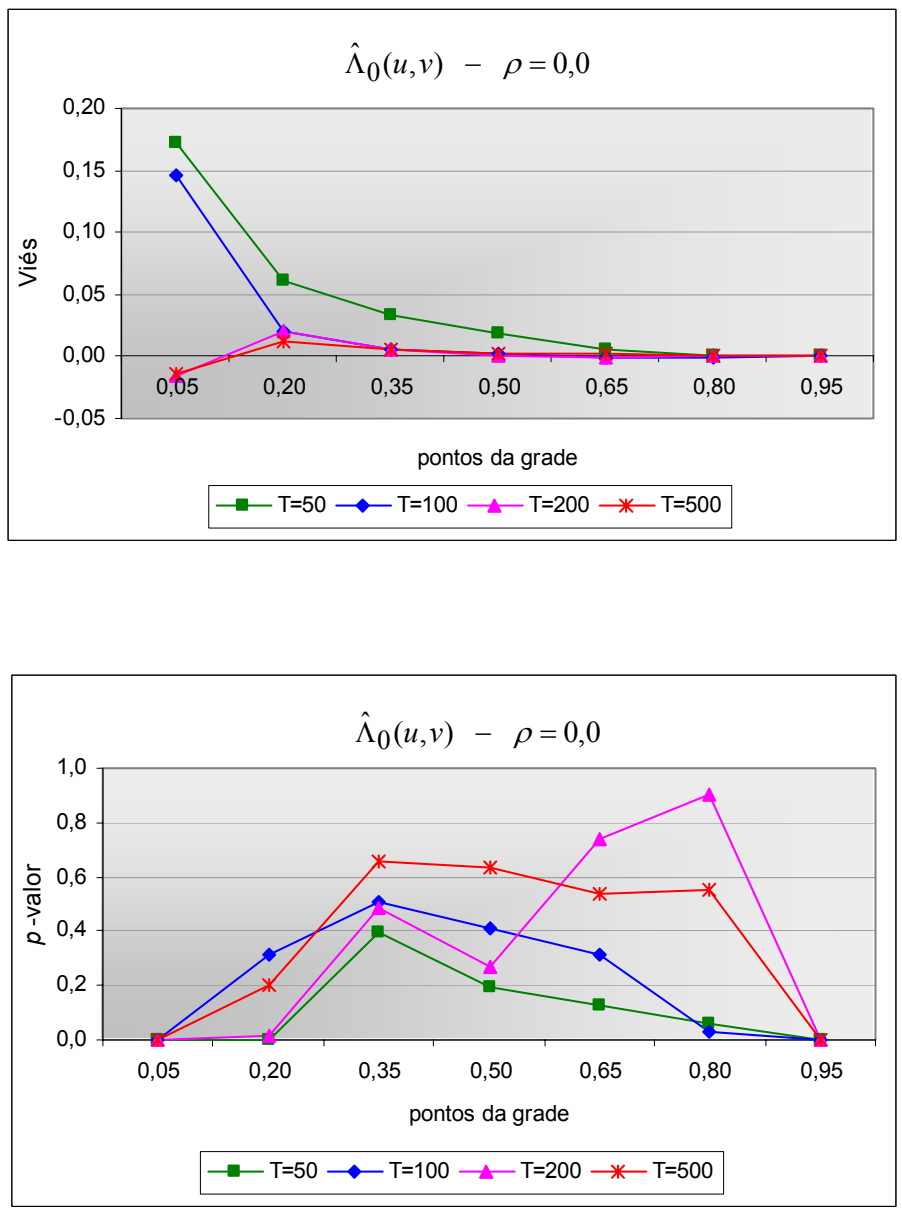

Figura D.8 - Função de dependência cruzada contemporânea local via cópula: gráfico de perspectiva e de curvas de nível estimadas para amostras de tamanho 50, além do comportamento do viés, erro quadrático médio e $p$-valor do teste de normalidade de Jarque Bera para diferentes tamanhos de amostras observadas de um modelo VAR(1) com média $(1,33 ; 4)^{\prime}$ e vec $(\Sigma)=(0,80 ; 0 ; 0 ; 2,86)^{\prime}($ correlação 0,0$)$. 
tau=1
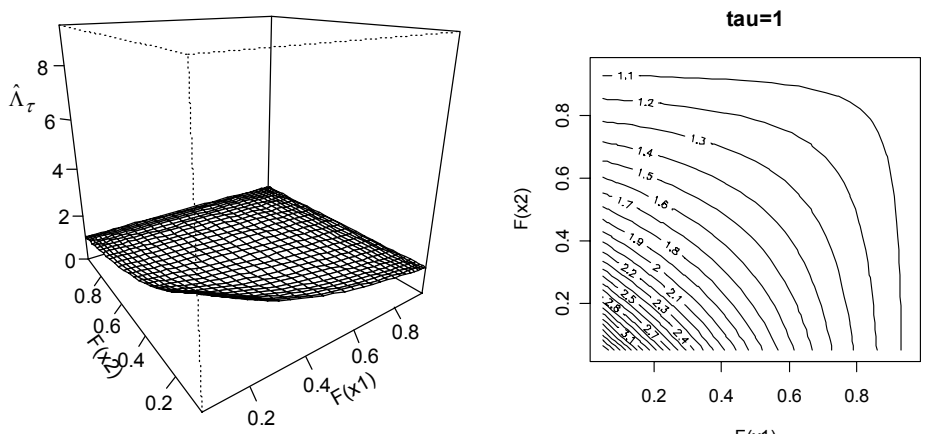

$\mathrm{F}(\mathrm{x} 1)$

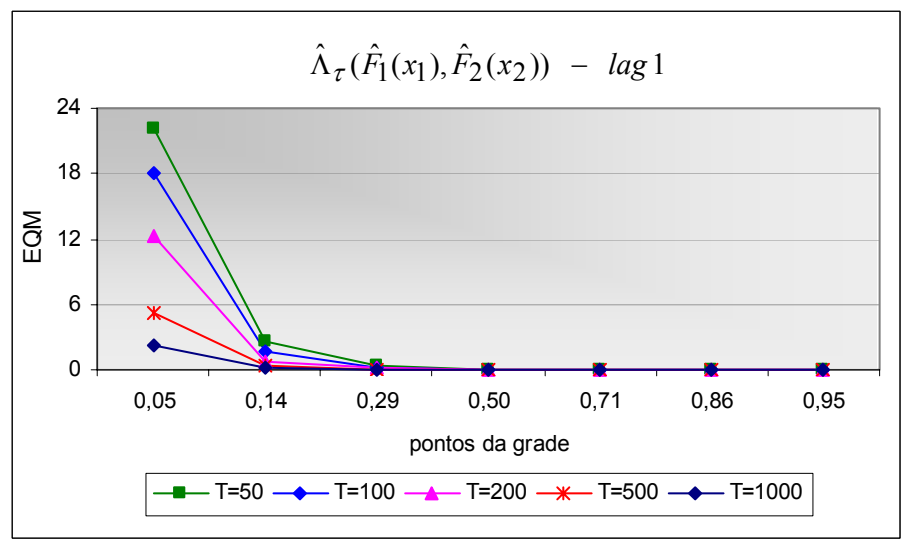

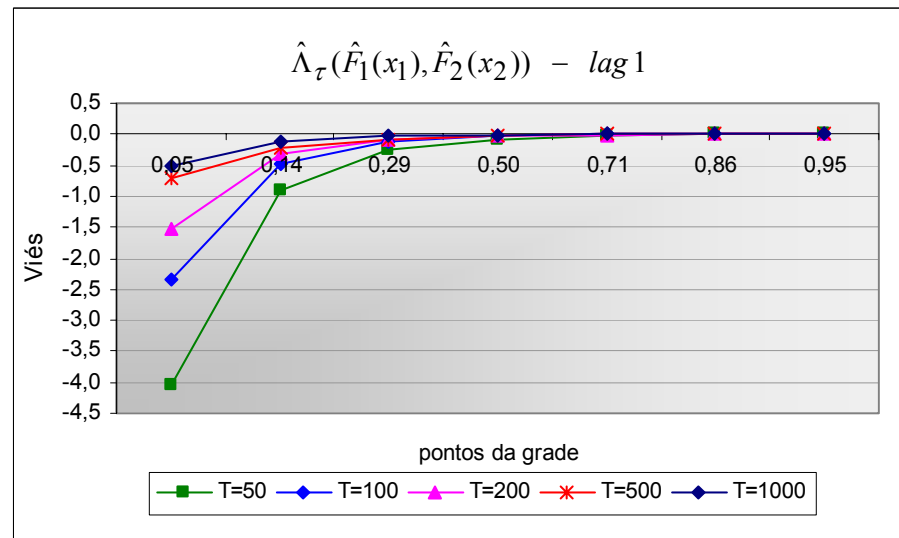

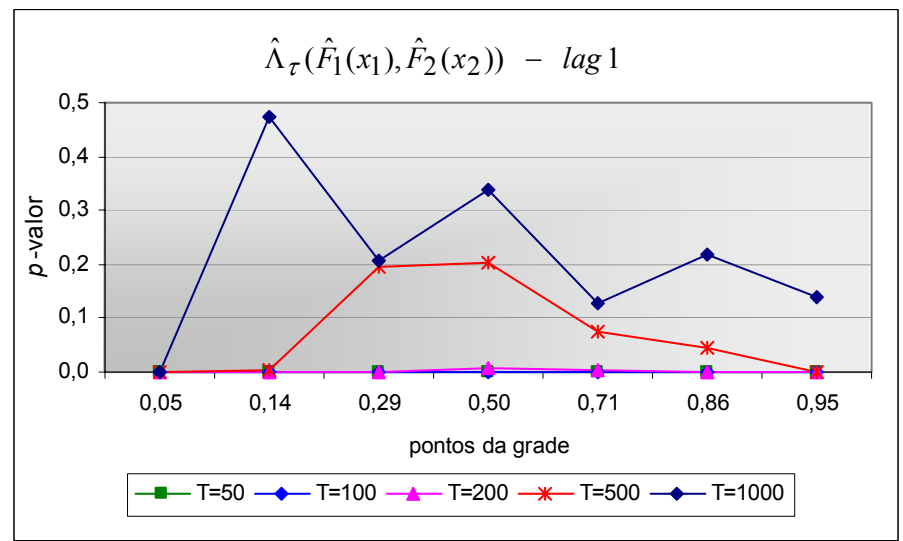

Figura D.9 - Função de autodependência local no lag 1, via funções de distribuição: gráfico de perspectiva e de curvas de nível estimadas para amostras de tamanho 50, além do comportamento do viés, erro quadrático médio e $p$-valor do teste de normalidade de Jarque Bera para diferentes tamanhos de amostras observadas de um processo Gaussiano com f.a.c. de um modelo AR(1) com $\phi_{1}=0,7$. 
tau=2
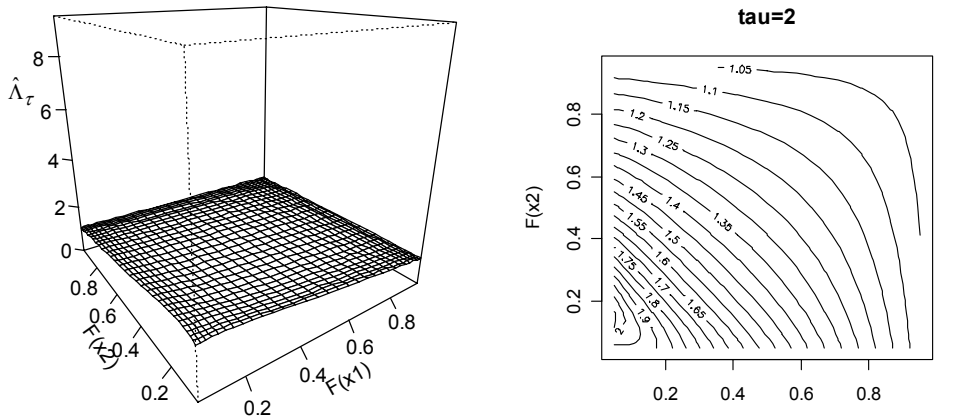

$\mathrm{F}(\mathrm{x} 1)$

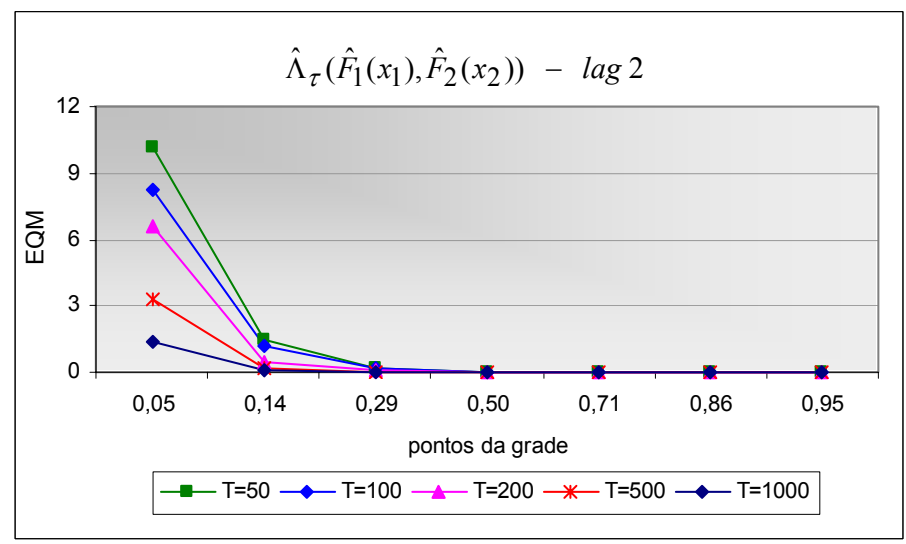

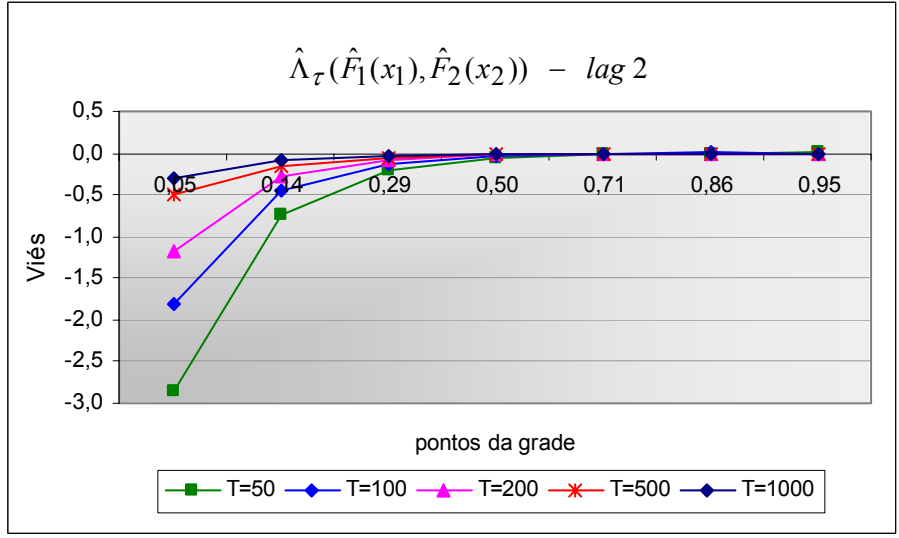

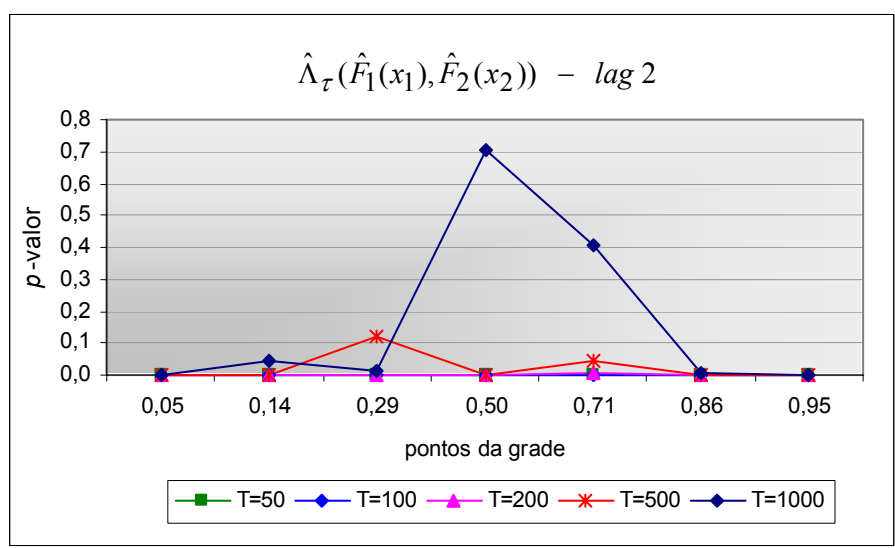

Figura D.10 - Função de autodependência local no lag 2, via funções de distribuição: gráfico de perspectiva e de curvas de nível estimadas para amostras de tamanho 50, além do comportamento do viés, erro quadrático médio e $p$-valor do teste de normalidade de Jarque Bera para diferentes tamanhos de amostras observadas de um processo Gaussiano com f.a.c. de um modelo AR(1) com $\phi_{1}=0,7$. 
tau=3
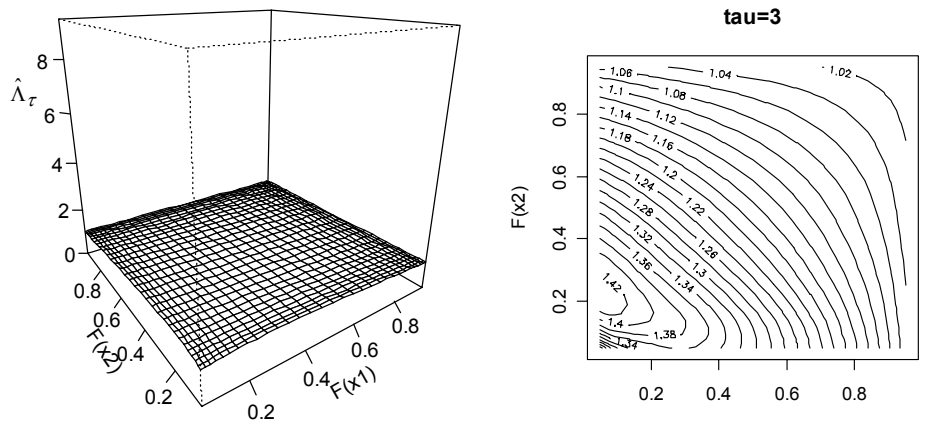

$\mathrm{F}(\mathrm{x} 1)$

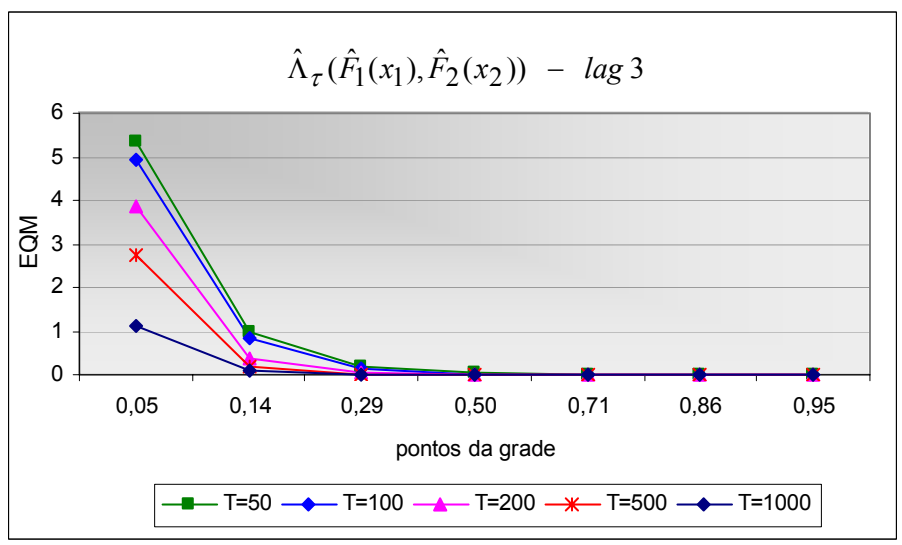

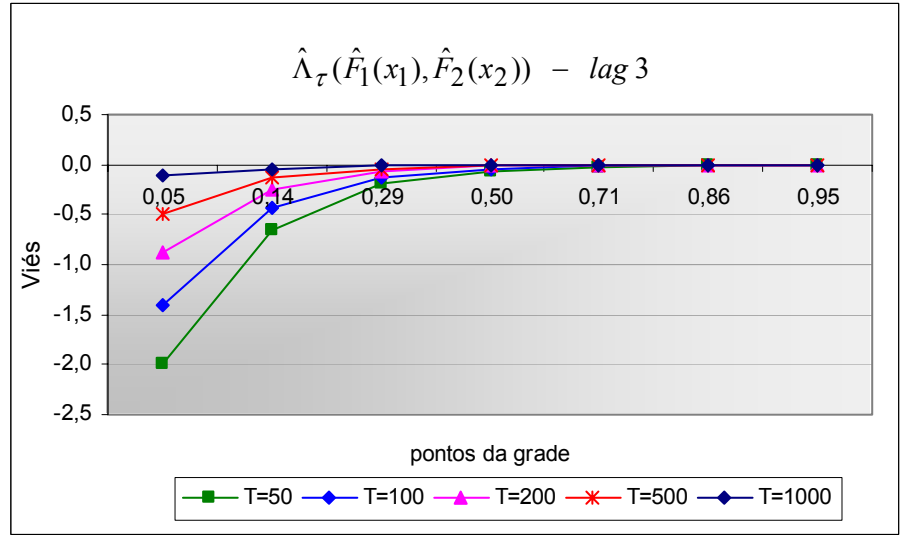

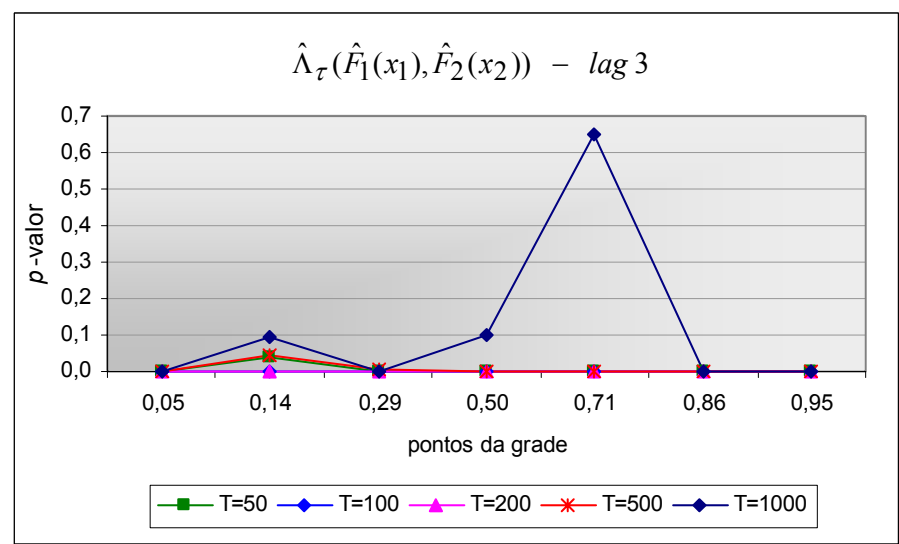

Figura D.11 - Função de autodependência local no lag 3, via funções de distribuição: gráfico de perspectiva e de curvas de nível estimadas para amostras de tamanho 50, além do comportamento do viés, erro quadrático médio e $p$-valor do teste de normalidade de Jarque Bera para diferentes tamanhos de amostras observadas de um processo Gaussiano com f.a.c. de um modelo AR(1) com $\phi_{1}=0,7$. 


\section{$\operatorname{tau}=4$}

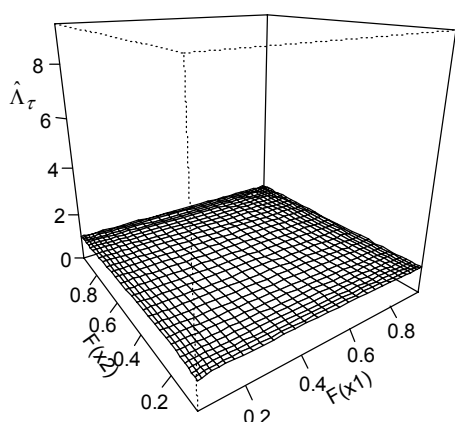

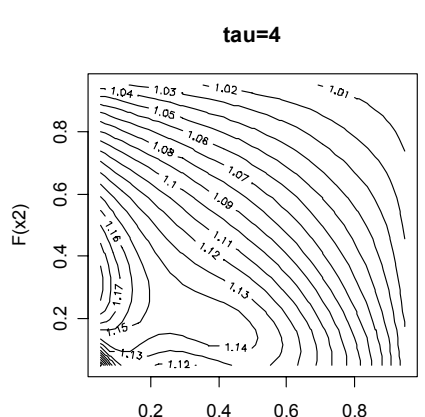

$F(x 1)$
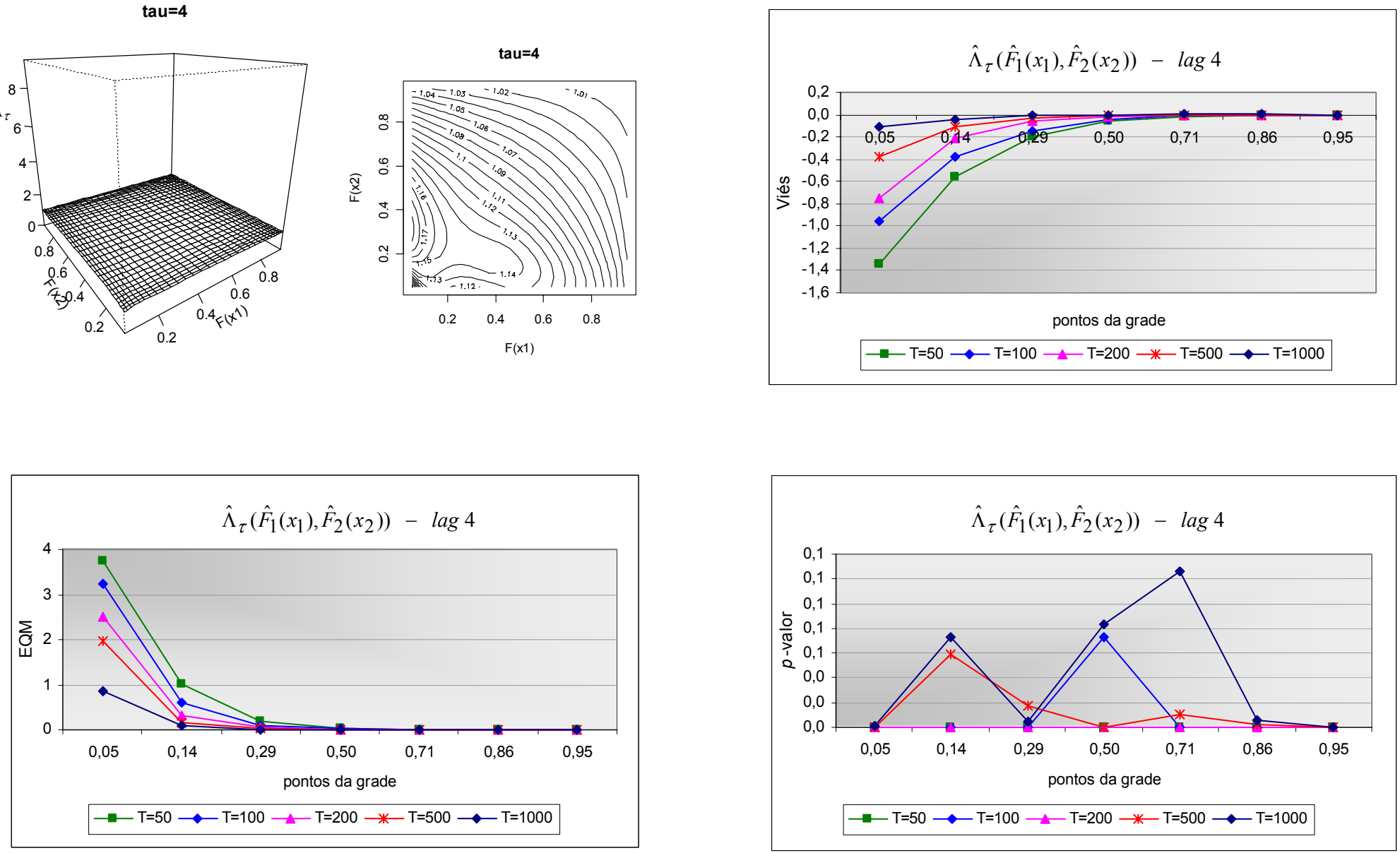

Figura D.12 - Função de autodependência local no lag 4, via funções de distribuição: gráfico de perspectiva e de curvas de nível estimadas para amostras de tamanho 50, além do comportamento do viés, erro quadrático médio e $p$-valor do teste de normalidade de Jarque Bera para diferentes tamanhos de amostras observadas de um processo Gaussiano com f.a.c. de um modelo AR(1) com $\phi_{1}=0,7$. 
tau $=1$

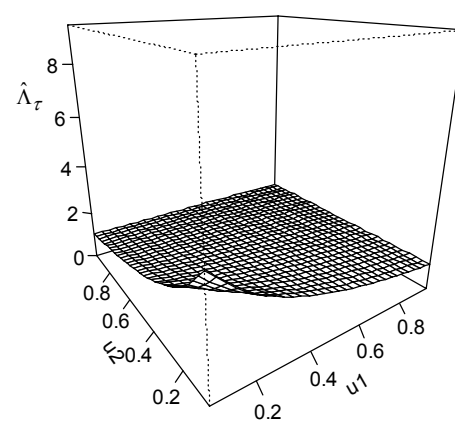

tau $=1$

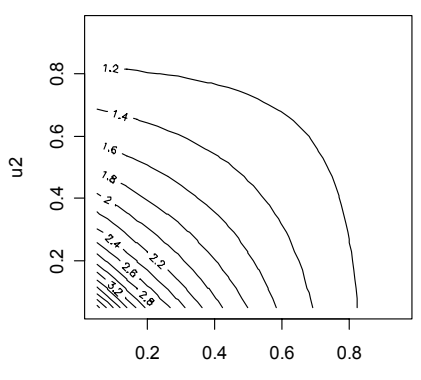

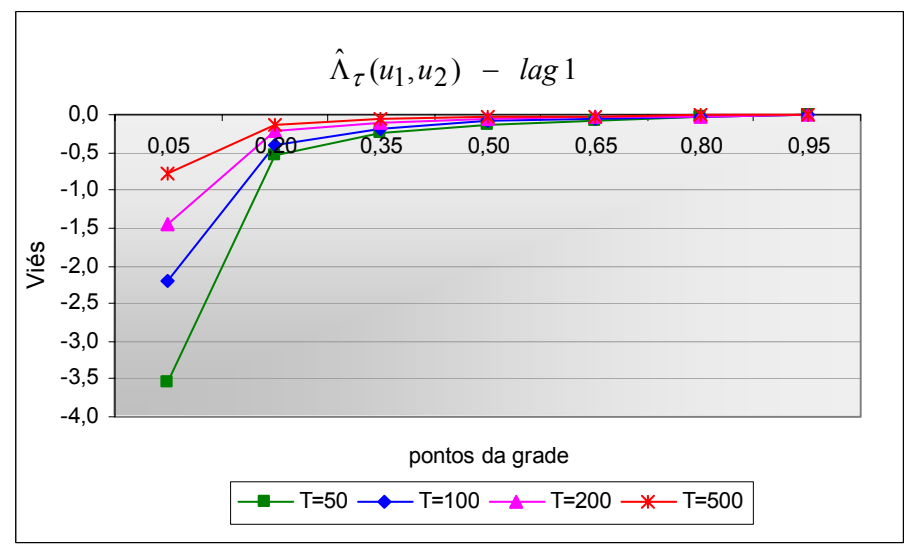

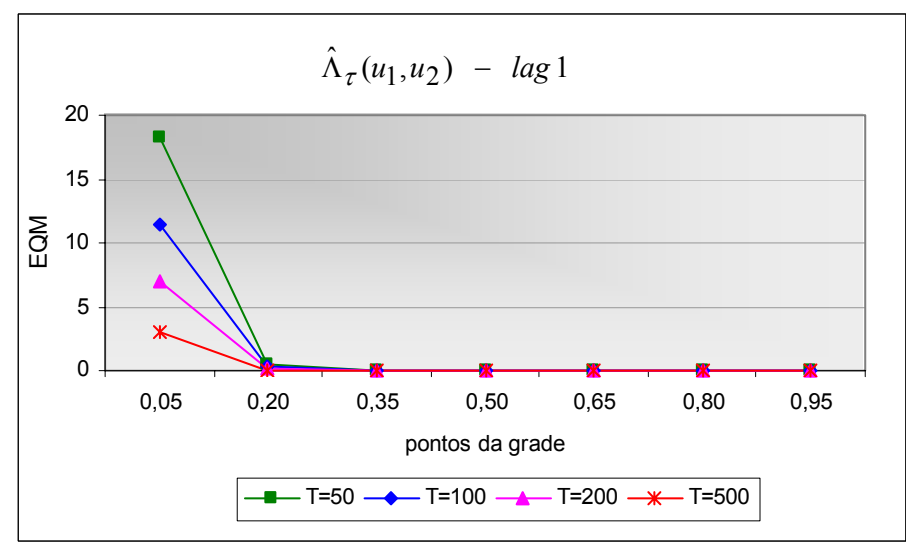

Figura D.13 - Função de autodependência local no lag 1, via cópula: gráfico de perspectiva e de curvas de nível estimadas para amostras de tamanho 50, além do comportamento do viés, erro quadrático médio e $p$-valor do teste de normalidade de Jarque Bera para diferentes tamanhos de amostras observadas de um processo Gaussiano com f.a.c. de um modelo $\operatorname{AR}(1) \operatorname{com} \phi_{1}=0,7$. 
tau=2

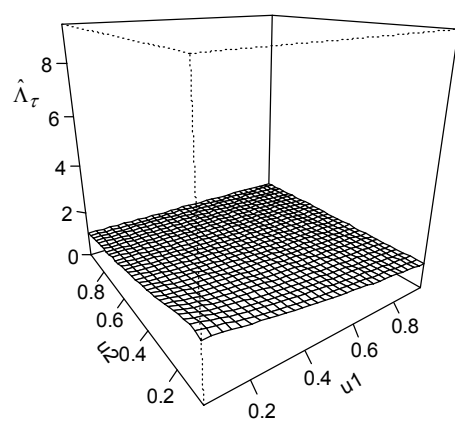

Э

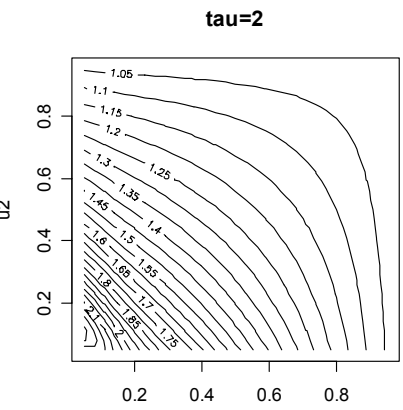

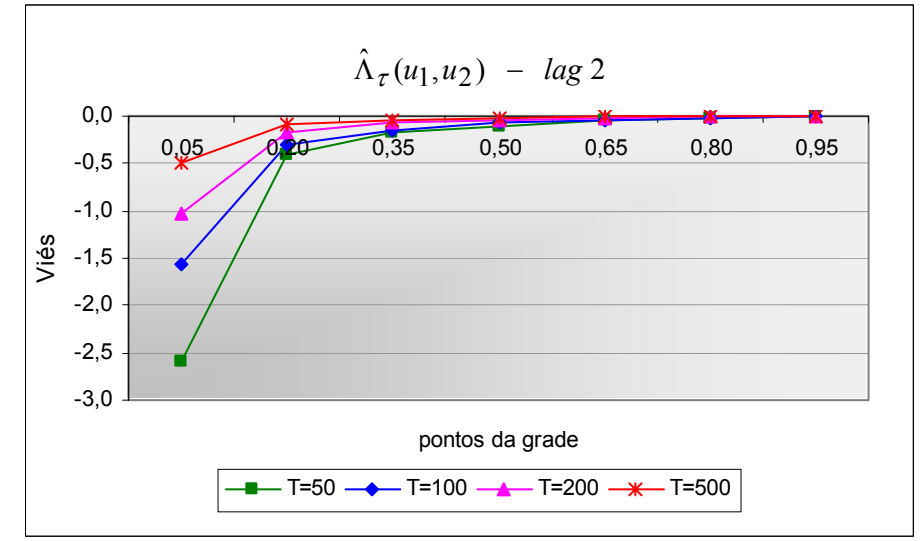
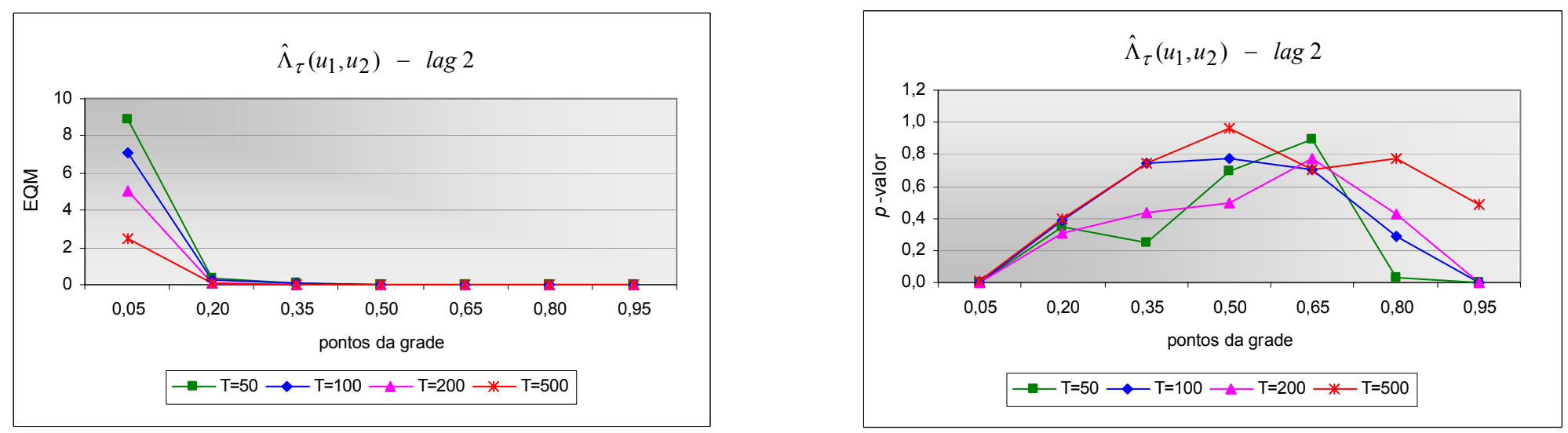

Figura D.14 - Função de autodependência local no lag 2, via cópula: gráfico de perspectiva e de curvas de nível estimadas para amostras de tamanho 50, além do comportamento do viés, erro quadrático médio e $p$-valor do teste de normalidade de Jarque Bera para diferentes tamanhos de amostras observadas de um processo Gaussiano com f.a.c. de um modelo $\operatorname{AR}(1) \operatorname{com} \phi_{1}=0,7$. 

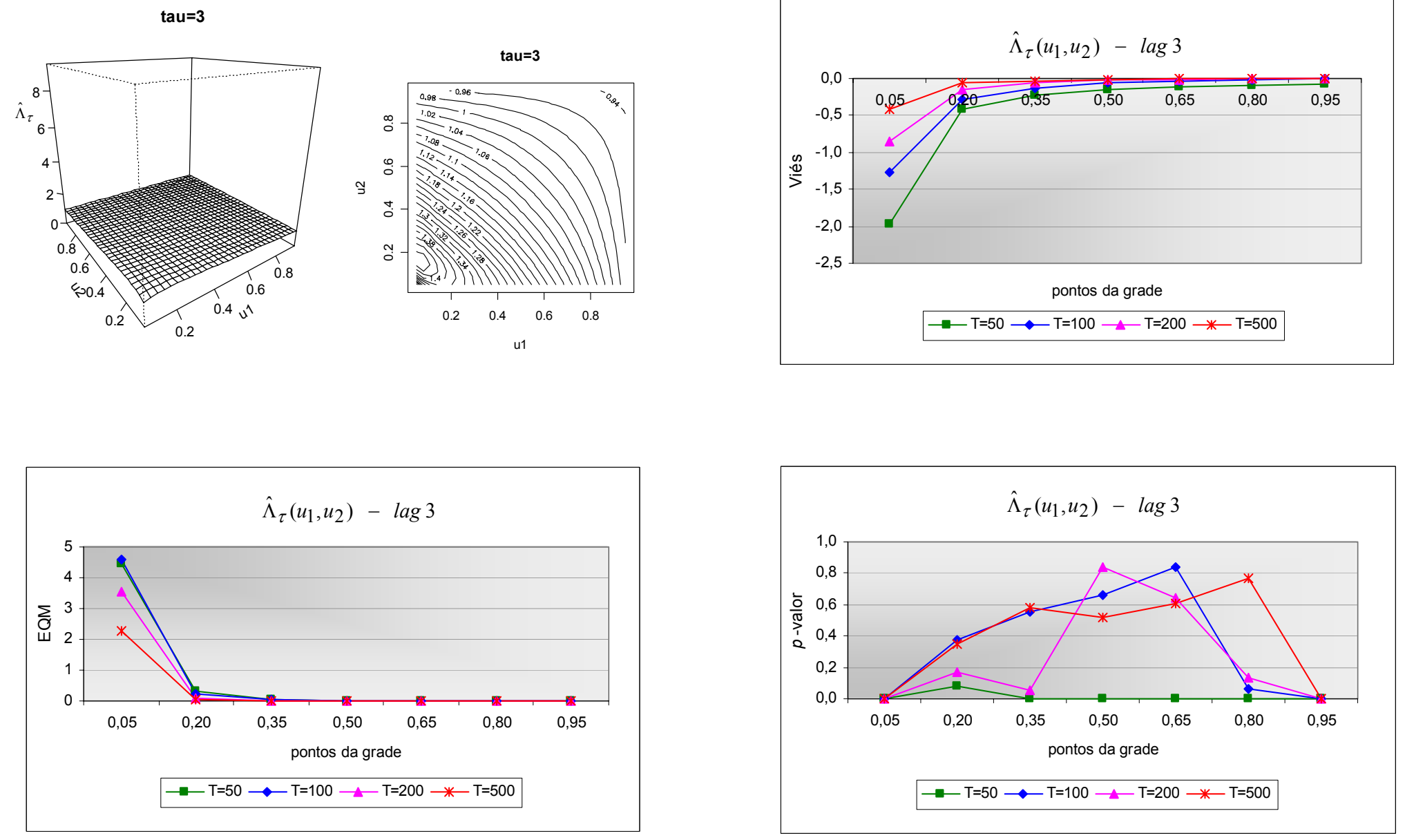

Figura D.15 - Função de autodependência local no lag 3, via cópula: gráfico de perspectiva e de curvas de nível estimadas para amostras de tamanho 50, além do comportamento do viés, erro quadrático médio e $p$-valor do teste de normalidade de Jarque Bera para diferentes tamanhos de amostras observadas de um processo Gaussiano com f.a.c. de um modelo $\operatorname{AR}(1) \operatorname{com} \phi_{1}=0,7$. 

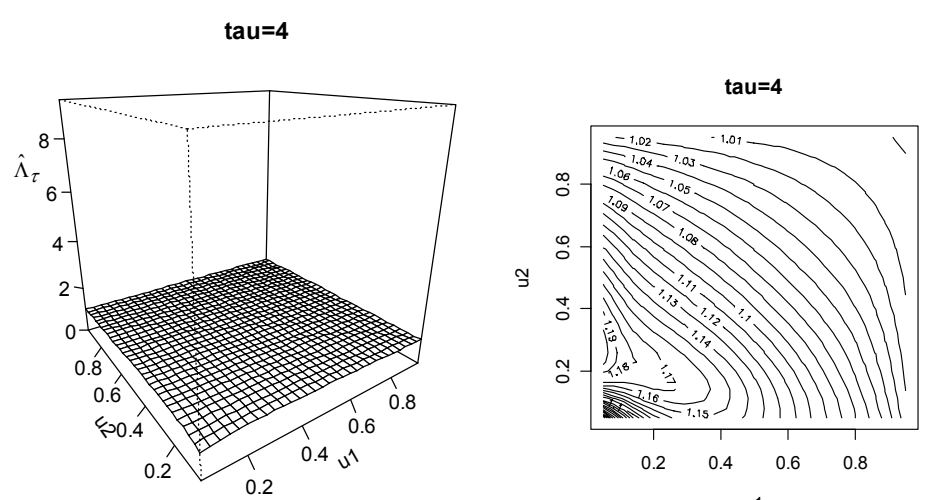

u1

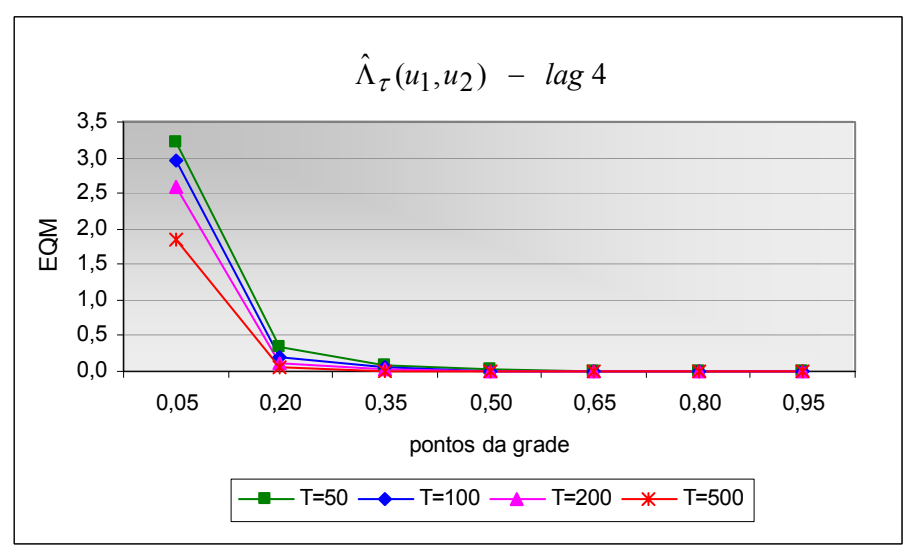

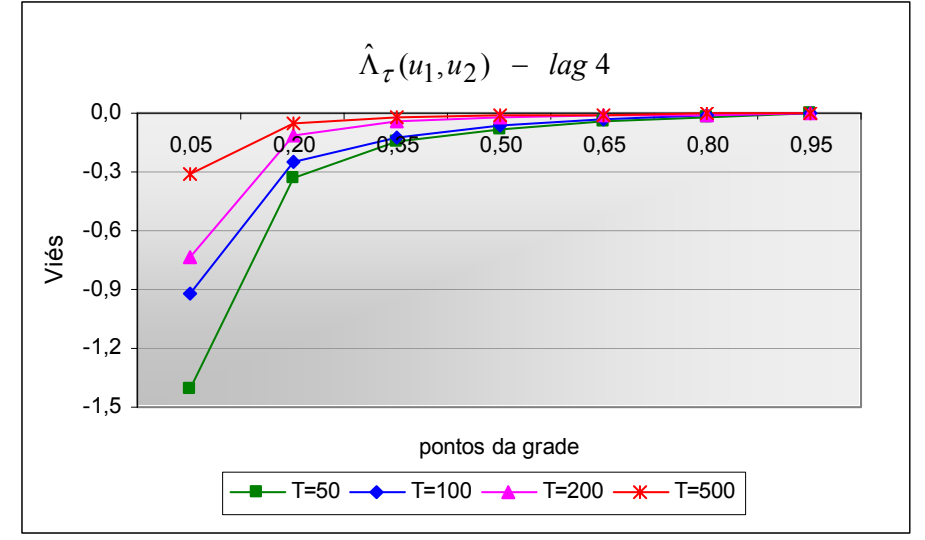

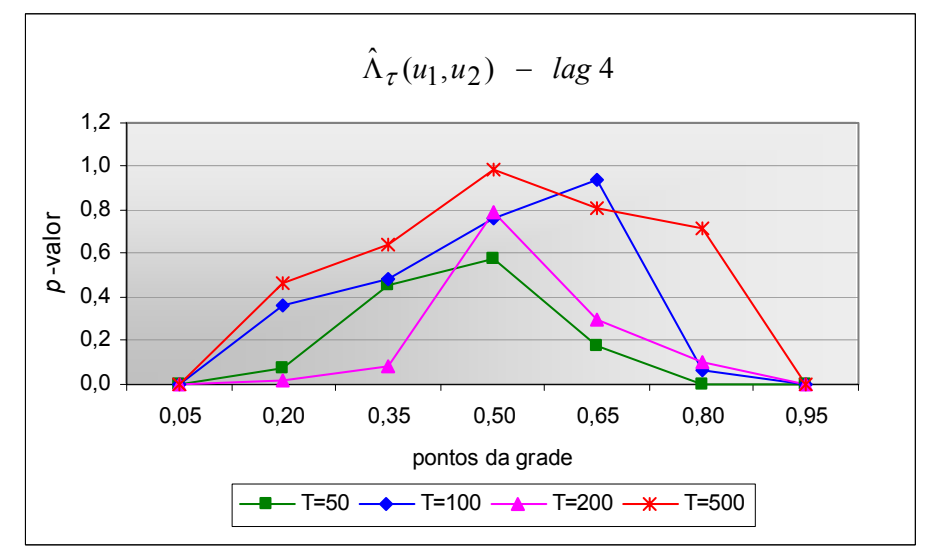

Figura D.16 - Função de autodependência local no lag 4, via cópula: gráfico de perspectiva e de curvas de nível estimadas para amostras de tamanho 50, além do comportamento do viés, erro quadrático médio e $p$-valor do teste de normalidade de Jarque Bera para diferentes tamanhos de amostras observadas de um processo Gaussiano com f.a.c. de um modelo $\operatorname{AR}(1) \operatorname{com} \phi_{1}=0,7$. 


\section{Referências Bibliográficas}

Azevedo, C., Oliveira, P. E. (2000). Kernel-type estimation of bivariate distribution function for associated random variables. New Trends in Probability and Statistics - Proceedings of the $6^{\text {th }}$ Tartu Conference, VSP, 5, 17-25.

Anjos, U. U., Kolev, N. V. (2005). Desenvolvimento e Análise de Estruturas de Dependência via Cópulas. Tese, IMEUSP.

Azzalini, A. (1981). A note on the estimation of a distribution function and quantiles by a kernel method. Biometrika, 68, 1, 326-328.

Bairamov, I., Kotz, S. and Kozubowski, T. J. (2003). A new measure of linear local dependence. Statistics, 37(3), 243258.

Bjerve, S. and Doksum, K. (1993). Correlation curves: Measures of association as functions of covariate values. Annals of Statistics, 21(2), 890-902.

Bosq, D. (1998). Nonparametric Statistics for Stochastic Process. Second Edition. New York: Springer-Verlag.

Doksum, K., Blyth, S., Bradlow, E., Meng, X., Zhao, H. (1994). Correlation Curves as Local Measures of Variance Explained by Regression. Journal of the American Statistical Association, 89(426), 571-582.

Engle, R. F. (1982). Autoregressive conditional heteroscedasticity with estimates of the variance of U.K. inflation. Econometrica, 50, 987-1008.

Fan, J. (1993). Local Linear regression smoothers and their minimax efficiencies. Annals of Statistics, 21, $196-216$.

Fan, J., Gijbels, I. (1995). Data-driven bandwidth selection in local polynomial fitting: Variable bandwidth and spatial adaptation. Journal of the Royal Statistical Society, Series B, 57, 371-394.

Fan, J., Gijbels, I. (1996). Local Polynomial Modelling and Its Applications. London: Chapman and Hall.

Fan, J., Yao, Q. (1998). Efficient estimation of conditional variance functions in stochastic regression. Biometrika, 85, 645-660.

Fan, J., Yao, Q. (2005). Nonlinear Time Series - nonparametric and parametric methods. New York: Springer.

Fermanian, J.-D., Scaillet, O. (2003). Nonparametric estimation of copulas for time series. Journal of Risk, 5, 25-54;

Fermanian, J. D., Radulovic, D., Wegkamp, M. (2004). Weak convergence of empirical copula processes. Bernoulli 10(5), 847-860.

Fuller, W. A. (1976). Introduction to Statistical Time Series. New York: John Wiley \& Sons.

Gasser, T., Müller, H. G. (1984). Estimating Regression Functions and Their Derivatives with Kernel Methods. Scandinavian Journal of Statistics, 11, 171-185.

Györfi, L., Härdle, W., Sarda, P., Vieu, P. (1989). Nonparametric Curve Estimation from Time Series. New York: Springer-Verlag.

Hansen, B. E. (2004). Bandwidth selection for nonparametric distribution estimation. Working paper.

Härdle, W. (1991). Smoothing Techniques: with implementation in S. New York: Springer Verlag.

Härdle, W., Lütkepohl, H., Chen, R. (1997). A review of nonparametric time series analysis. International Statistical Review, 65(1), 49-72.

Holland, P. W. and Wang, Y. J. (1987). Dependence Function for Continuous Bivariate Densities. Communications in Statistics Theory and Methods, 16(3), 863-876.

Jones, M. C. (1990). The performance of kernel density functions in kernel distribution function estimation. Statistics \& Probability Letters, 9, 129-132.

Kolev. N. V., Gonçalves, M. Dimitrov, B. (2007). Probabilistic Properties of Sibuya's Dependence Function. Preprint. 
Kotz, S., Nadarajah, S. (2003). Local dependence functions for elliptically symmetric distributions. Sankhyã, 65(1), 207223.

Lehmann, E. L. (1999). Elements of Large-Sample Theory. New York: Springer.

Masry, E., Tjøstheim, D. (1995). Non-parametric estimation and identification of non-linear ARCH time series. Econometric Theory, 11, 258-289.

Morettin, P. A., Toloi, C. M. C., Chiann, C., de Miranda, J. C. S. (2006). Wavelet estimation of copulas for time series. Preprint.

Nadarajah, S., Mitov, K., Kotz, S. (2003). Local dependence functions for extreme value distributions. Journal of Applied Statistics, No. 10, December, 1081-1100.

Nadaraya, E. A. (1964). On estimating regression. Theory Probab. Appl., 9, 141-142.

Nelsen, R. B. (2007). An Introduction to Copulas. Second Edition. New York: Springer-Verlag.

Rényi, A. (1959). On measures of dependence. Acta Math. Acad. Sci. Hungar., 10, 441-451.

Ruppert, D., Sheather, S. J., Wand, M. P. (1995). An effective bandwidth selector for local least squares repression. Journal of the American Statistical Association, 90, 1257-1270.

Silverman, B. W. (1986). Density Estimation for statistics and data analysis. London: Chapman and Hall.

Sklar, A. (1959). Fonctions de répartition à n dimensions e leurs marges. Publications de l'Institut de Statistique de l'Université de Paris 8, 229-231.

Sibuya, M. (1960). Bivariate Extreme Statistics. Annals of the Institute of Statistical Mathematics, 11, 195-210.

Yang, L., Härdle, W., Nielsen, J. P. (1999). Nonparametric autoregression with multiplicative volatility and additive mean. Journal of Time Series Analysis, 20, 579-604.

Wand, M. P., Jones, M. C. (1995). Kernel Smoothing. New York: Wiley.

Watson, G. S. (1964). Smooth regression analysis. Sankhyã Ser. A., 26, 359-372. 